Copyright: The author, Maria Mylova, 2020.

\title{
Gravitational Waves from Inflation
}

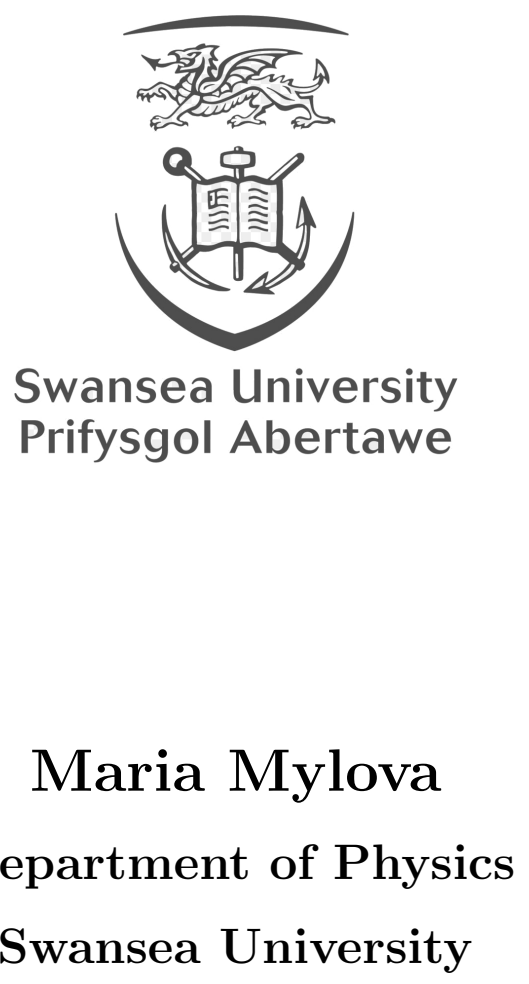

Submitted to Swansea University in fulfilment of the requirements for the degree of Doctor of Philosophy

February 2020 


\section{Abstract}

The focus of this work is on the topic of gravitational wave cosmology. We look for new observational signatures for primordial gravitational waves and especially for frameworks that can produce an enhanced tensor spectrum. In such cases, the gravitational wave energy density can enter within the sensitivity curves of gravitational wave detectors or it could leave a measurable imprint in the polarization pattern of the Cosmic Microwave Background radiation. This is very important as more sensitive, next generation experiments are expected to make indirect and direct measurements of the primordial stochastic gravitational wave background.

Our investigation showed that an enhanced tensor amplitude can be achieved, within the framework of the Horndeski theory, if there is a short period where slowroll inflation is violated. We considered a model of kinetically driven inflation where for a short time the scalar field velocity is heavily time-dependent. During that time the would-be decaying tensor mode becomes a growing mode. Analysis of the bispectrum showed that this can lead to sizeable tensor non-Gaussianities.

We also considered possible realizations of the effective field theory of ScalarTensor gravity. In particular, we looked for a setup up of operators that could lead to the enhancement of parity violating effects for tensors. We introduced modifications to gravity which enabled us to parametrically approach the scale at which maximal parity violation occurs. Using the mathematical machinery for effective field theories we showed that sub-leading quadratic operators can become important signalling the presence of the Chern Simons instability. This inevitably implies the existence of non-trivial cubic interactions which could stand the theory strongly coupled at energies not far above the energy scale of inflation. Consequently, this can lead to large parity violation in tensor non-Gaussianity. 


\section{Suggested Layout of Declaration/Statements page}

\section{DECLARATION}

This work has not previously been accepted in substance for any degree and is not being concurrently submitted in candidature for any degree.

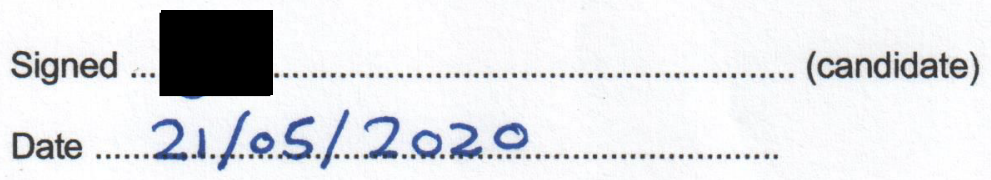

\section{STATEMENT 1}

This thesis is the result of my own investigations, except where otherwise stated. Where correction services have been used, the extent and nature of the correction is clearly marked in a footnote(s).

Other sources are acknowledged by footnotes giving explicit references. A bibliography is appended.

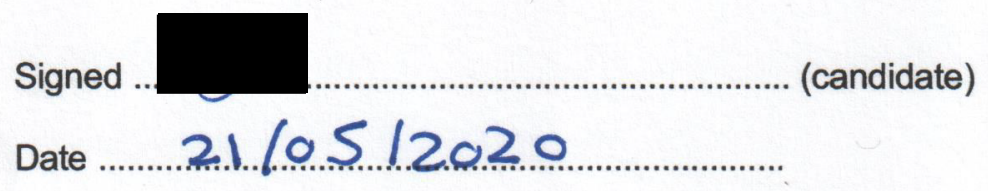

\section{STATEMENT 2}

I hereby give consent for my thesis, if accepted, to be available for photocopying and for inter-library loan, and for the title and summary to be made available to outside organisations.

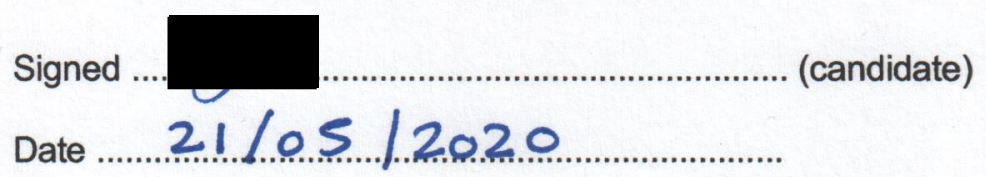

NB: Candidates on whose behalf a bar on access has been approved by the University (see Note 7), should use the following version of Statement 2:

I hereby give consent for my thesis, if accepted, to be available for photocopying and for inter-library loans after expiry of a bar on access approved by the Swansea University.

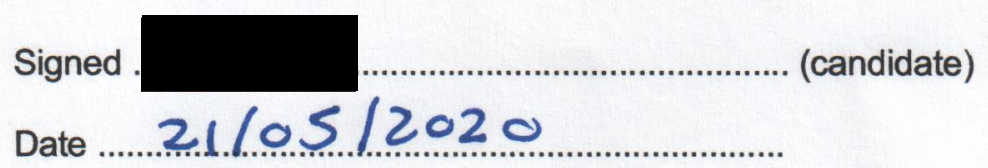




\section{Acknowledgements}

I would like to thank my supervisor Gianmassimo Tasinato for always being helpful and for providing me with guidance, especially on technical as well more philosophical matter regarding my work. I am grateful to Carlos Núñez and Ivonne Zavala Carrasco for very useful discussions and comments on my work. I am deeply indebted to Steven Walter for his continuous support and encouragement throughout my postgraduate education. Finally, many thanks to Gonzalo A. Palma for carefully reviewing my latest manuscript. This work was funded by an STFC studentship under the DTP ST/N504464/1.

There are known knowns, there are known unknowns, but there are also unknown unknowns.

Donald Rumsfeld

Unfortunately, although the answer was indeed clear, simple, and straightforward, there is some difficulty in justifiably assigning to it the fourth of the epithets you applied to the statement, inasmuch as the precise correlation between the information you communicated and the facts, insofar as they can be determined and demonstrated, is such as to cause epistemological problems, of sufficient magnitude as to lay upon the logical and semantic resources of the English language a heavier burden than they can reasonably be expected to bear.

Sir Humphrey (Yes, Prime Minister, BBC) 


\section{Contents}

$\begin{array}{lll}\text { List of Figures } & \text { VI }\end{array}$

1 The theory of inflation 3

1.1 Introduction to General Relativity . . . . . . . . . . . . . . . . . 4

1.1.1 Variational approach to General Relativity . . . . . . . . . . 6

1.1.2 The Einstein field equations . . . . . . . . . . . . . 6

1.2 The expanding universe . . . . . . . . . . . . . . . . . . . . . . . . . . 8

1.3 Inflation from a single scalar field . . . . . . . . . . . . . . . 12

2 Introduction to primordial gravitational waves $\quad 19$

2.1 Linearised gravity . . . . . . . . . . . . . . . . . . 20

2.2 Tensor polarizations . . . . . . . . . . . . . . . . . . 22

2.3 Primordial gravitational waves . . . . . . . . . . . . . . . . . . 24

2.4 Detecting primordial gravitational waves . . . . . . . . . . . . . 28

2.5 Non-gaussianity . . . . . . . . . . . . . . . . . . . . . 35

3 Beyond Einstein gravity $\quad 41$

3.1 Bottom-up approach . . . . . . . . . . . . . . . . . . . . 41

3.2 Extended theories of gravity . . . . . . . . . . . . . . . . . . . . 43

4 Chiral primordial gravitational waves in extended theories of Scalar$\begin{array}{lr}\text { Tensor gravity } & 48\end{array}$

4.1 Abstract . . . . . . . . . . . . . . . . . . . . . . 48

4.2 Introduction . . . . . . . . . . . . . . . . . . . . . . . 48

4.3 Parametric amplification of chiral primordial gravitational waves . . . 51

4.3.1 Disformally transformed action . . . . . . . . . . . 52

4.3.2 Validity of the EFT . . . . . . . . . . . . . . . 54

4.3.3 The equations of motion . . . . . . . . . . . . 56

4.4 On the stability of parity breaking theories . . . . . . . . . . . . 59

4.5 A toy model . . . . . . . . . . . . . . . . . . . . . . . . . . . . . . . 60

4.5.1 Chirality enhancement . . . . . . . . . . . . 62

4.6 Relation between frames . . . . . . . . . . . . . . . . . 63

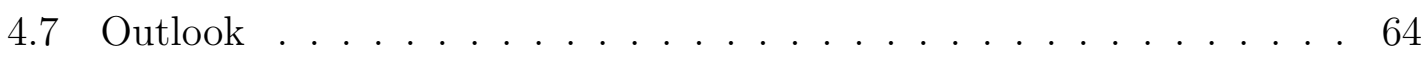


$\begin{array}{llr}5 & \text { Non-attractor inflation } & \mathbf{6 6}\end{array}$

5.1 Abstract . . . . . . . . . . . . . . . 6 66

5.2 Introduction . . . . . . . . . . . . . . . . . 66

5.3 Formulation . . . . . . . . . . . . . . . . . . . 69

5.4 Tensor duality . . . . . . . . . . . . . . . . . . . 70

5.4.1 The tensor dual of a slow-roll phase . . . . . . . . . . . . 72

5.5 Amplifying tensor modes and realising the tensor duality . . . . . . . 73

5.5 .1 Our aims ....................... 73

5.5 .2 The model . . . . . . . . . . . . . . . . . 75

5.5.3 Background evolution . . . . . . . . . . . . . 76

5.5.4 Dynamics of fluctuations . . . . . . . . . . . . . . 79

5.6 Enhancement of fluctuations . . . . . . . . . . . . . . . . . . 81

5.7 Numerical example . . . . . . . . . . . . . . . . . . . . . . . . . . . 82

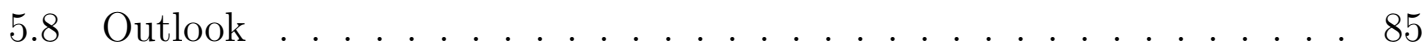

6 Non-Gaussianity in non-attractor inflation $\quad 87$

6.1 Abstract . . . . . . . . . . . . . . . 87

6.2 Introduction . . . . . . . . . . . . . . . . . . . . . 87

6.3 The two-point function . . . . . . . . . . . . . . . . . . . . 88

6.4 Tensor non-Gaussianity in non-attractor inflation . . . . . . . . . . 91

6.5 Disformal transformations and tensor non-Gaussianity . . . . . . . . . . . . . . . . . . . . . . . . . . . . . 98

6.6 Conclusions . . . . . . . . . . . . . . . . . . . . 101

$\begin{array}{lr}\text { A Appendix } & 102\end{array}$

A.1 Covariant energy-momentum conservation law . . . . . . . . . . . . . 102

A.2 Variation of the inverse metric . . . . . . . . . . . . . . . . 103

A.3 Coordinate transformation . . . . . . . . . . . . . . . . . 103

A.4 Friedmann Equations . . . . . . . . . . . . . . . . . . . . 104

A.5 Scalar field equations of motion . . . . . . . . . . . . 106

A.6 Perturbations in de Sitter spacetime . . . . . . . . . . . . . . . . 107

A.7 Weyl tensor squared . . . . . . . . . . . . . . . . . . . . . 108

A.8 Weyl tilde squared . . . . . . . . . . . . . . . . . . . . . . 110

A.9 Disformal transformation of linearised (NLO) operators . . . . . . . . 111

A.10 The equations of motion . . . . . . . . . . . . . . 115

A.11 Disformal transformation of covariant (NLO) operators . . . . . . . . 118

A.12 Next-to-next-to leading order operators . . . . . . . . . . . . . . 120

A.13 Disformal transformation of the tensor action . . . . . . . . . . . . 122

A.14 The "GR" contribution . . . . . . . . . . . . . . . . . . . . . 124

A.15 The Exponential Integral . . . . . . . . . . . . . . . . . 131

$\begin{array}{ll}\text { Bibliography } & 133\end{array}$ 


\section{List of Figures}

1.1 The history of the Universe. Image credit: BICEP2 2014 Release Image Gallery . . . . . . . . . . . . . . . . . . . 4

1.2 Inflaton rolling down its potential [1]. The amount of inflation is controlled by the "flatness" of the potential. The excursion of the field between horizon exit and the end of inflation is denoted by $\Delta \phi . .12$

1.3 A comparison to theoretical predictions for inflationary models with data from Planck alone and in combination with BK14 or BK14 plus $B A O$ data, for $r$ and $n_{s}$ at the pivot scale $k=0.002 / M p c^{-1}$ [2]. . . .

2.1 Distortion of a ring of particles by a gravitational wave travelling perpendicularly to the plane of the page. The image depicts the two polarizations of gravitational waves: plus (top) and cross (bottom). Image Credit: Wm. Robert Johnston, UT Dallas. . . . . . . . . . . . 23

2.2 The B-mode pattern, thought to be generated by gravitational waves in the CMB, which was observed with the BICEP2 telescope. The twisting behaviour of the B-mode pattern in clockwise or anti-clockwise directions is shown in red and blue colour. Image credit: BICEP2 2014 Release Image Gallery . . . . . . . . . . . . . . . . . . . .

2.3 The first map shows the uniform temperature of the CMB, as measured by COBE, while the second shows the dipole anisotropy $\Delta T / T \sim$ $10^{-3}$ at $l=1$ due to the motion of the Earth relative to the frame of the CMB. The third map includes emissions from the Milky way but the dipole contribution has been subtracted. It shows the CMB anisotropies for $l>1$ at $\Delta T / T \sim 10^{-5}$. Image credit: COBE Image Gallery.

2.4 The Planck (2015) temperature power spectrum [3]. The vertical axis displays the quantity $\mathcal{D}_{l}=l(l+1) \mathcal{C}_{l} / 2 \pi$. The multipole moments $l$ are displayed on the horizontal axis. The scale is treated logarithmically till $l=50$ and linearly after that. Large angles correspond to small $l$. The error bars show uncertainties of $\pm 1 \sigma$ due to cosmic variance. We use the peaks to extact information about the cosmological parameters. The damping tail at high multipoles is because decoupling of photons from baryons does not happen instantaneously. . . . . .

2.5 The mechanism that produces $E$ - and B-modes in the CMB. Image credit: BICEP2 2014 Release Image Gallery . . . . . . . . . . . . . . 
2.6 Here we see the sensitivity of LISA to a stochastic GW background relative to other experiments [4]. The observational predictions from inflation, maximum blue-tilted spectrum and electroweak phase transition are indicated with red, blue and green dashed lines. Current and future sensitivity of experiments is indicated by black and grey curves. The sensitivities displayed are from LIGO and Virgo, aLIGO, PTA and $S K A$. On the left of the diagram we can see the sensitivity for current and future $C M B$ experiments where the black curve shows the current bound for $r<0.1$, while the grey curve shows the projected sensitivity for future $C M B$ experiments $r<0.001$. Finally, the sensitivity to measurements from Big Bang Nucleosynthesis is displayed by the indirect curve.

2.7 Shapes of the bispectrum [5] with momenta satisfying $\mathbf{k}_{3} \leq \mathbf{k}_{2} \leq \mathbf{k}_{1}$.

4.1 Once the parameter $c_{T}$ attains the value in (4.3.15) cubic interactions (in blue) become more important than the quadratic ones (in red), signalling the break down of the effective description. For this plot we take $M_{P l}=10^{18} \mathrm{GeV}$, and $H=10^{13} \mathrm{GeV}$. The horizontal axis displays the values of $c_{T}$ in the interval $\left\{10^{-4}, 10^{-3}\right\}$ which is the range in which we expect to find the value for $c_{T}$ in (4.3.15) for this particular numerical example, while the vertical axis displays the energy expansion ratio at the non-relativistic limit $E_{\text {ratio }} \sim \mathcal{O}(1)$.

5.1 Schematic behaviour of the scalar field derivative in our system. Notice the intermediate non-attractor phase $\dot{\phi} \sim 1 / a^{3}$. During the entire inflationary evolution the background geometry corresponds to pure de Sitter space. Image credit: G. Tasinato.

5.2 The behaviour of $\dot{\phi}$ and $\left|h_{i j}\right|$ through the transition to the non-attractor phase where we have used the expressions (5.5.12) and (5.3.10), respectively. On the right, we have set $\mathcal{C}_{2}=\mathcal{C}_{1}\left(H_{0} / c_{T}\right)$ with $\mathcal{C}_{1}=10^{-7}$ in (5.3.10) to show the evolution of $\left|h_{i j}\right|$. For both plots we have the following choice of parameters: $\alpha_{1}=1, \beta_{1}=-1, \sigma_{1}=2.5, \rho_{1}=$ $1, \delta_{1}=0, \alpha_{2}=10^{-3}, \beta_{2}=1.5, \sigma_{2}=-0.2, \delta_{2}=-4, \rho_{2}=-4.3$ where $N=0$ corresponds to the transition point. In these plots the non-attractor phase lasts approximately $\Delta N \simeq 2.7$ e-folds. 
6.1 We show qualitatively how primordial tensor modes get amplified during a non-attractor phase. They can contribute to the $G W$ energy density $\Omega_{G W}$ and thus enter within the sensitivity curves for $G W$ detectors in their appropriate frequency ranges (expressed in $\mathrm{Hz}$ ). We model inflation as a pure de Sitter phase, during which a short period of non-attractor evolution occurs - whose starting time and duration depend on the model one considers - enhancing the tensor spectrum. We use formula (5.6.5) and assume for simplicity instantaneous transitions between attractor and non-attractor eras. Our conventions for the definition of the $G W$ energy density $\Omega_{G W}$ are the same as in [6]. Image credit: Plots by Cari Powell. . . . . . . . . . . . . . . . . . . 89

$6.2 \mathcal{A}_{\text {(new) }}^{+++}\left(1, x_{2}, x_{3}\right) /\left(x_{2} x_{3}\right)$ as a function of $x_{2}$ and $x_{3}$ where we set $H \mu^{\mathrm{end}} / \mathcal{G}_{T}^{\mathrm{end}} \rightarrow$ 1 (Left). $\mathcal{A}_{(\mathrm{GR})}^{+++}\left(1, x_{2}, x_{3}\right) /\left(x_{2} x_{3}\right)$ as a function of $x_{2}$ and $x_{3}$ (Right). In both plots we normalized the amplitudes $\mathcal{A}_{\text {(new) }}^{+++}$and $\mathcal{A}_{(\mathrm{GR})}^{+++}$to unity for equilateral configurations $x_{2}=x_{3}=1$ and took $-K y_{\mathrm{end}}=10^{-2}$. Image credit: Plots by O. Ozsoy. . . . . . . . . . . . . . . . . . 98 


\section{Introduction}

The focus of this work is to look for theoretical frameworks, in the context of singlefield inflation, which can lead to new observational signatures for tensors [7-9]. Next generation of experiments will signal a new era for early universe cosmology. More sensitive CMB experiments, such as LiteBIRD, SPIDER and CMBpol, will measure the B-Mode polarization anisotropies with expected precision $r \sim 10^{-3}[10,11]$. A direct detection of a primordial stochastic gravitational wave background may be possible with space-based interferometers, such as LISA, DECIGO (Deci-Hertz) or their proposed successor BBO (Big Bang Observer) $[6,12,13]$, as well with PTAs (Pulsar Timing Arrays) [14]. A definitive detection will provide evidence of the inflationary paradigm and most importantly reveal the energy scale of inflation. This can help to constrain a number of cosmological parameters and consequently to exclude non-viable inflationary models.

A potential obstacle to making a successful detection is that conventional inflationary scenarios predict a very small tensor amplitude. Our research focuses on finding ways to overcome these limitations by considering inflationary scenarios with much richer phenomenology. We especially focus on frameworks that can produce an enhanced tensor spectrum. In such cases the gravitational wave energy density can enter within the sensitivity curves of gravitational wave detectors or leave a measurable imprint in the CMB polarization pattern. Together with my collaborators we showed that this can be achieved, within the framework of the Horndeski theory, if there is a short period where slow-roll inflation is violated [8]. Furthermore we analysed the bispectrum and found this can lead to sizeable tensor non-Gaussianities [9].

In more recent work [7], we considered possible realizations of the effective field theory (EFT) of scalar-tensor gravity. The upper bound to the energy scale of inflation, from the Planck collaboration [15], is currently at $H \lesssim 10^{13} \mathrm{GeV}$, so it is reasonable to wonder whether inflation could be sensitive to high energy physics.

In particular, we looked for a setup up of operators that could lead to the enhancement of parity violating effects for tensors. This is challenging to achieve in the context of single-field inflation as theoretical predictions tend to suffer quantitatively, due to the Chern Simons instability [16], leading to a negligible chirality enhancement. To re-examine this problem in a more systematic way we introduced modifications to gravity which enabled us to parametrically approach the scale at which maximal parity violation occurs. Using the mathematical machinery for effective field theories we showed that sub-leading quadratic operators can become important signalling the presence of the Chern Simons instability. This inevitably 
implies the existence of non-trivial cubic interactions which could stand the theory strongly coupled at energies not far above the energy scale of inflation. Consequently, this can lead to large parity violation in tensor non-Gaussianity. This is a great insight as we can use this as an opportunity to try and identify what are the necessary changes in the physical description of the system that will enable us, in a consistent manner, to maintain sufficient parity violation while ensuring the stability of the modes. This translates to finding a weakly coupled description of the system so that we can consistently parametrize the physics as we extrapolate it to higher energies.

The thesis is organized as follows. In Chapter (1) we introduce the theory of inflation and briefly explain how it solves some of they key problems within the Big Bang theory. In Chapter (2) we briefly review the theory of gravitational waves and their observational consequences in the Cosmic Microwave Background (CMB). In Chapter (3) we look at effective field theories (EFT) of gravity, from the bottom-up point of view, as well modifications to gravity that lead to second-order equations of motion for gravitational waves but change the dispersion relation of the fluctuations, such as the Horndeski theory. In Chapter (4) we re-examine the problem of parity violation in single-filed inflation by looking at extensions, from the effective field theory point of view, of theories with a non-trivial dispersion relation for the graviton. There we show that the Chern Simons instability is a consequence of the theory being strongly-coupled. This means that new physics may appear not far above the energy scale of inflation which could lead to a maximally left-handed gravitational wave signal at the end of inflation and consequently to large parity violation in tensor non-Gaussianity. In Chapter (5 ) we develop a framework in terms of kinetically driven single-field inflation where for a short time the scalar field velocity is heavily time-dependent. During this phase, which we dub as a non-attractor inflationary phase, the would-be decaying tensor mode grows outside the horizon leading to the enhancement of tensor fluctuations. Finally in Chapter (6) we investigate primordial tensor non-Gaussianity during the phase of non-attractor evolution. We find that the amplitude of the tensor bispectrum is enhanced at the squeezed limit with respect to slow-roll models. Finally, we include several technical Appendices. 


\section{The theory of inflation}

A successful theory of the early universe could offer scientists a deeper understanding on the physical processes which take part at energy scales that we cannot attain with any terrestrial experiment, such as particle accelerators. In the absence of a complete theory of quantum gravity, we can only reasonably speculate about physics below the scale of the reduced Planck mass $\left(M_{P l} \sim 18 \mathrm{GeV}\right)$. The theory of inflation is the result of bringing together our best knowledge from particle physics and Einstein's theory of General Relativity (GR). This enables us to develop theoretical models that can predict what happened in the first moments of the Big Bang. We cross reference these early universe models with data from the Cosmic Microwave Background and by accounting for their effect on subsequent epochs in the evolution of the universe.

Inflation was introduced in the seminal paper by Guth in 1981 [17] and later on improved by Starobinsky [18], Linde [19], Albrecht and Steinhardt [20]. It has gained popularity ever since and grew to become the well established research field which goes under the specialized tag of inflationary cosmology. Among other successes the inflationary paradigm offers solutions to some key problems within the Big Bang theory, namely the flatness problem, the horizon problem and the monopole problem, by postulating a period, in the early universe, of accelerated expansion of spacetime. This is how we think that all causally disconnected regions of spacetime came to be under one common horizon. As well, due to the rapid expansion the universe grew enough so that the apparent curvature of spacetime, at large scales, appears to be flat.

The simplest model that describes this process if that of a single scalar field slowly rolling down its potential till it reaches the bottom where it starts to oscillate, causing the universe to expand [21]. At the end of this process reheating commences while the scalar field decays into other particles. Our physical measurements start when the Universe was 380.000 years old, as indicated in Figure (1.1), at the time of decoupling where photons were able to escape and reach us as the Cosmic Microwave Background radiation. These photons appear to arrive on Earth evenly from every direction forming a uniform sphere around the observer known as the surface of last scattering. The temperature of the CMB photons in the present epoch is measured to be $T_{0} \approx 2.7 \mathrm{~K}$. The CMB temperature contains small temperature fluctuations known as anisotropies which are thought to be responsible for structure formation in the universe $[22,23]$. Additionally, a small percentage of the CMB radiation is expected to be weakly polarized due to Thomson scattering of photons by free electrons. Of particular interest, to this work, are the B-Modes which if they are detected this will be solid proof of primordial gravitational waves. Next we give a 
brief introduction to the theory of General Relativity (GR).

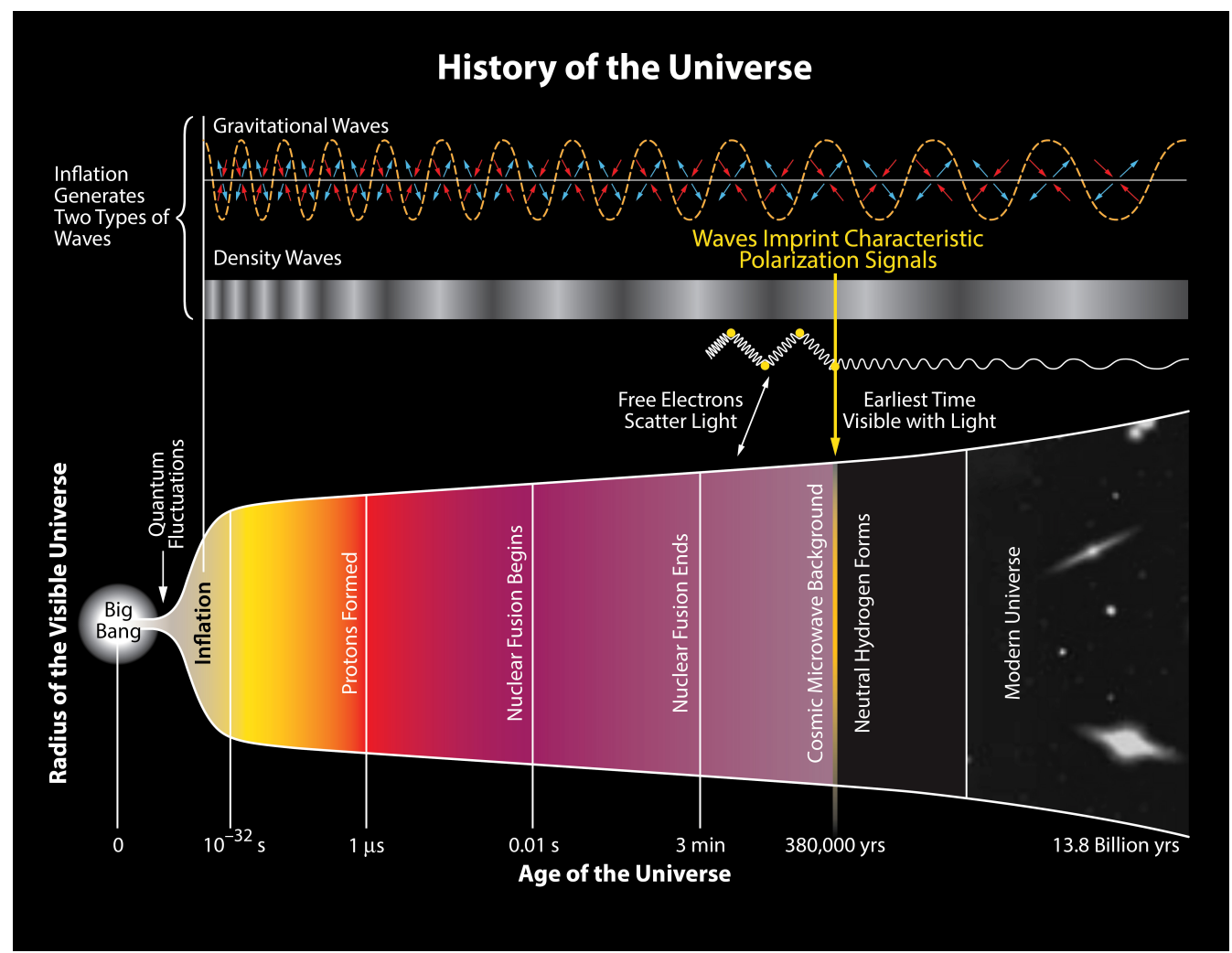

Figure 1.1: The history of the Universe. Image credit: BICEP2 2014 Release Image Gallery

\subsection{Introduction to General Relativity}

Below we introduce some of the basic ingredients that we will use throughout this work. We employ the sign conventions in [24] unless otherwise stated. The Minkowski metric signature is given by $\eta_{\mu \nu}=\operatorname{diag}(-,+,+,+)$. The metric $g_{\mu \nu}$, with inverse $g^{\mu \nu}$, describes the geometric and gravitational properties of spacetime. Natural units apply throughout the document with $c=\hbar=G=1$ and the Einstein summation is omitted, unless is otherwise stated. Covariant differentiation may be denoted by a semicolon, i.e. $\nabla_{\mu} \equiv_{; \mu}$ while partial differentiation may be denoted by a comma, i.e. $\partial_{\mu} \equiv_{, \mu}$.

Covariant derivatives are defined in terms of partial derivatives plus a correction, which is given in terms of the connection coefficient $\Gamma_{\mu \sigma}^{\rho}[25]$

$$
\nabla_{\mu} V^{\nu}=\partial_{\mu} V^{\nu}+\Gamma_{\mu \lambda}^{\nu} V^{\lambda}
$$

The Christoffel symbols of the first-kind are defined as

$$
\Gamma_{\nu \alpha \beta}=\frac{1}{2}\left(\partial_{\alpha} g_{\nu \beta}+\partial_{\beta} g_{\alpha \nu}-\partial_{\nu} g_{\alpha \beta}\right),
$$


while the Christoffel symbols of the 2nd-kind are obtained by rising the first index

$$
\Gamma_{\alpha \beta}^{\mu}=g^{\mu \nu} \Gamma_{\nu \alpha \beta}=\frac{1}{2} g^{\mu \nu}\left(\partial_{\alpha} g_{\nu \beta}+\partial_{\beta} g_{\alpha \nu}-\partial_{\nu} g_{\alpha \beta}\right) .
$$

In terms of the above one can derive a useful formula for the divergence of a vector [26], given by

$$
\nabla_{\mu} V^{\nu}=\frac{1}{\sqrt{g}} \partial_{\mu}\left(\sqrt{g} V^{\mu}\right)
$$

The Riemann curvature tensor is defined as

$$
R_{\beta \gamma \delta}^{\alpha}=\partial_{\gamma} \Gamma_{\beta \delta}^{\alpha}-\partial_{\delta} \Gamma_{\beta \gamma}^{\alpha}+\Gamma_{\mu \gamma}^{\alpha} \Gamma_{\beta \delta}^{\mu}-\Gamma_{\mu \delta}^{\alpha} \Gamma_{\beta \gamma}^{\mu},
$$

and has the following useful properties; antisymmetry in the first and second index and third and fourth index, i.e. $R_{\mu \nu \rho \sigma}=R_{\nu \mu \rho \sigma}=-R_{\mu \nu \sigma \rho}$. It is symmetric under exchange of first and second pairs of indices, i.e. $R_{\mu \nu \rho \sigma}=R_{\rho \sigma \mu \nu}$. Other useful properties are swapping indices, i.e. $R_{\mu \nu \rho}^{\sigma} A_{\sigma}=R_{\sigma \mu \nu \rho} A^{\sigma}$ and $R_{\rho \mu \nu}^{\rho}=0$. The Ricci tensor is obtained by contracting two indices of the Riemann tensor $R_{\mu \nu} \equiv R_{\mu \lambda \nu}^{\lambda}$, and it is symmetric under exchange of indices

$$
R_{\beta \delta} \equiv R_{\beta \alpha \delta}^{\alpha}=\partial_{\alpha} \Gamma_{\beta \delta}^{\alpha}-\partial_{\delta} \Gamma_{\beta \alpha}^{\alpha}+\Gamma_{\mu \alpha}^{\alpha} \Gamma_{\beta \delta}^{\mu}-\Gamma_{\mu \delta}^{\alpha} \Gamma_{\beta \alpha}^{\mu} .
$$

From this, the Ricci scalar is obtained by contracting the remaining two indices

$$
R=g^{\beta \delta} R_{\beta \delta}=\partial_{\alpha} \Gamma_{\delta}^{\alpha \delta}-\partial_{\delta} \Gamma_{\alpha}^{\alpha \delta}+\Gamma_{\mu \alpha}^{\alpha} \Gamma_{\delta}^{\mu \delta}-\Gamma_{\mu \delta}^{\alpha} \Gamma_{\alpha}^{\mu \delta} .
$$

Some useful identities that involve the Riemann tensor are:

\section{The cyclic identity:}

$$
R_{[\nu \rho \sigma]}^{\mu}=R_{\nu \rho \sigma}^{\mu}+R_{\rho \sigma \nu}^{\mu}+R_{\sigma \nu \rho}^{\mu}=0 .
$$

The Ricci identity:

$$
\left(\nabla_{\mu} \nabla_{\nu}-\nabla_{\nu} \nabla_{\mu}\right) A_{\rho}=R_{\rho \nu \mu}^{\lambda} A_{\lambda}
$$

The second Bianchi identity:

$$
\nabla_{\lambda} R_{\rho \sigma \mu \nu}+\nabla_{\rho} R_{\sigma \lambda \mu \nu}+\nabla_{\sigma} R_{\lambda \rho \mu \nu}=0 .
$$

The contracted Bianchi identity:

$$
R_{\lambda . \rho}^{\rho}=\frac{1}{2} R_{. \lambda} \Rightarrow 2 R_{\lambda . \rho}^{\rho}=R_{. \lambda} .
$$

Note, partial derivatives always commute, i.e. $\partial_{\sigma} \partial_{\rho} g_{\mu \nu}=\partial_{\rho} \partial_{\sigma} g_{\mu \nu}$. 


\subsubsection{Variational approach to General Relativity}

The Lagrangian formalism is widely used in the formulation of classical and quantum field theories. It helps to identify conserved quantities present in the system and to derive the equations of motion. This is achieved with the use of a variational principle, by imposing that the action integral of the system has to remain stationary under any variations of the field variables that the Lagrangian of the theory depends on.

In this Section we consider an asymptotically flat spacetime, meaning that the field variables vanish at infinitely large spatial distances. The action is evaluated by using a boundary that it is assumed to be at a very large radius.

Finally, it is useful to include the following formulae; the Euler-Lagrange equations for an arbitrary number of derivatives are given by

$$
-\frac{\partial \mathcal{L}}{\partial X}+\partial^{\alpha}\left\{\frac{\partial \mathcal{L}}{\partial\left(\partial^{\alpha} X\right)}\right\}-\partial^{\beta} \partial^{\alpha}\left\{\frac{\partial \mathcal{L}}{\partial\left(\partial^{\beta} \partial^{\alpha} X\right)}\right\}+\cdots=0 .
$$

In terms of partial derivatives $\left(\partial_{\eta}, \partial_{k}, \partial_{m}, \cdots\right)$, then we have

$$
\begin{aligned}
& -\frac{\partial \mathcal{L}}{\partial X}+\partial^{\eta}\left\{\frac{\partial \mathcal{L}}{\partial\left(\partial^{\eta} X\right)}\right\}+\partial^{k}\left\{\frac{\partial \mathcal{L}}{\partial\left(\partial^{k} X\right)}\right\}-\partial^{\eta} \partial_{\eta}\left\{\frac{\partial \mathcal{L}}{\partial\left(\partial^{\eta} \partial_{\eta} X\right)}\right\} \\
& -\partial^{k} \partial^{m}\left\{\frac{\partial \mathcal{L}}{\partial\left(\partial^{k} \partial^{m} X\right)}\right\}-\partial^{\eta} \partial^{k}\left\{\frac{\partial \mathcal{L}}{\partial\left(\partial^{\eta} \partial^{k} X\right)}\right\}+\cdots \\
& +\partial^{k} \partial^{m} \partial^{l}\left\{\frac{\partial \mathcal{L}}{\partial\left(\partial^{k} \partial^{m} \partial^{l} X\right)}\right\}+\cdots-\partial^{k} \partial^{m} \partial^{l} \partial^{n}\left\{\frac{\partial \mathcal{L}}{\partial\left(\partial^{k} \partial^{m} \partial^{l} \partial^{n} X\right)}\right\} \\
& +\cdots=0 .
\end{aligned}
$$

\subsubsection{The Einstein field equations}

In the classical theory of General Relativity, one is interested to find an action which describes gravity as a manifestation of the curvature of spacetime [27]. This is achieved with the use of the Einstein-Hilbert action

$$
S_{E H}\left[g_{\mu \nu}\right]=\frac{1}{16 \pi G} \int \mathrm{d} v_{x} g^{\mu \nu} R_{\mu \nu},
$$

where $G$ is Newton's gravitational constant and $\mathrm{d} v_{x}=\mathrm{d}^{4} x \sqrt{|g|}$ is the invariant volume element in four spacetime dimensions. The Lagrangian scalar density $\mathcal{L}=$ $\sqrt{|g|} L=\sqrt{|g|} g^{\mu \nu} R_{\mu \nu}$ depends on components of the metric tensor and derivatives of it. The metric is treated as a dynamic field variable defined on a 4-dimensional manifold $\mathcal{M}$. The Ricci scalar $R=g^{\mu \nu} R_{\mu \nu}$ is the simplest scalar quantity that one can derive from the metric tensor.

We can produce the Einstein field equations (EFE) by varying the action with respect to variations of the metric, demanding that the variation vanishes at the 
boundaries $(\delta S=0)$. The integration is taken to be over a 4 -dimensional volume, where the fixed endpoints are hypersurfaces of constant time $t$, in which case the boundary terms are integrals over a spatial region $\mathcal{R}$ [25]. Variation of the action with respect to variations of the metric $g^{\mu \nu}$ and minimizing (Appendix A.2) leads to the Einstein field equations for vacuum [28], which read

$$
\frac{\delta S_{E H}}{\delta g^{\mu \nu}}=(16 \pi G)^{-1} G_{\mu \nu}=(16 \pi G)^{-1}\left(R_{\mu \nu}-\frac{1}{2} g_{\mu \nu} R\right)=0,
$$

where $G_{\mu \nu}=R_{\mu \nu}-\frac{1}{2} g_{\mu \nu} R$ is the Einstein tensor, which is symmetric under exchange of indices $G_{\mu \nu}=G_{\nu \mu}$.

The gravitational action $S_{E H}$ in vacuum does not depend on matter fields and thus the left-hand side (LHS) of the Einstein field equations will be comprised by geometric terms, while the right-hand side (RHS) is equal to zero. In the presence of matter fields, one can obtain the non-vacuum Einstein field equations by simply adding an extra term to the Einstein-Hilbert action

$$
S=S_{E H}+S_{M}
$$

The variation of the matter action $S_{M}$ with respect to variations of the metric, reads

$$
\delta S_{M}=\int \mathrm{d}^{n} x \frac{\delta S_{M}}{\delta g_{\mu \nu}} \delta g_{\mu \nu},
$$

where the energy momentum tensor (EMT) of the fields is defined as

$$
T_{\mu \nu} \equiv-\frac{2}{\sqrt{|g|}} \frac{\delta S_{M}}{\delta g^{\mu \nu}}
$$

where $\frac{\delta S_{M}}{\delta g_{\mu \nu}(x)}$ is a functional derivative and the minus sign is due to the mostly "plus" metric signature convention used here. The stress-tensor is symmetric under exchange of indices $T_{\mu \nu}=T_{\nu \mu}$ and covariantly conserved (Appendix A.2)

$$
\nabla_{\mu} T^{\mu \nu}=0
$$

This way one recovers the full Einstein field equations (EFE) in the presence of matter

$$
\delta S=\delta S_{E H}+\delta S_{M}=0 \Rightarrow G_{\mu \nu}=k T_{\mu \nu}
$$

where $k=8 \pi G$. The normalization factor $8 \pi$ ensures that we can obtain the correct Newtonian limit. In the case where the matter action is comprised of the kinetic term of a single scalar field and a potential $U(\phi)$ which can contain mass and interaction terms, we have

$$
S=\int \mathrm{d}^{n} x \sqrt{|g|} L=\frac{1}{2} \int \mathrm{d}^{n} x \sqrt{|g|}\left[g^{\alpha \beta} \partial_{\alpha} \phi \partial_{\beta} \phi+U(\phi)\right] .
$$

Then using (1.1.18), gives

$$
T_{\mu \nu}=\partial_{\mu} \phi \partial_{\nu} \phi-g_{\mu \nu} L .
$$


Therefore, the EFE becomes

$$
\begin{aligned}
R_{\mu \nu}-\frac{1}{2} g_{\mu \nu} R & =(8 \pi G)\left[\partial_{\mu} \phi \partial_{\nu} \phi-g_{\mu \nu} L\right] \\
& =(8 \pi G)\left[\partial_{\mu} \phi \partial_{\nu} \phi-g_{\mu \nu} g^{\alpha \beta} \partial_{\alpha} \phi \partial_{\beta} \phi-g_{\mu \nu} U(\phi)\right] .
\end{aligned}
$$

We can approximate the matter in the Universe at large scales with a perfect fluid with pressure $p$, energy density $\rho$ and velocity $u_{\mu}$, where $u^{\mu}$ is a time-like vector $\left(u_{\mu} u^{\mu}=-1\right)$. This way the EMT is expressed as

$$
T_{\mu \nu}=(\rho+p) u_{\mu} u_{\nu}+p g_{\mu \nu} .
$$

In the presence of a cosmological constant $\Lambda$, the EFE are given by

$$
R_{\mu \nu}-\frac{1}{2} R g_{\mu \nu}+\Lambda g_{\mu \nu}=8 \pi G T_{\mu \nu}
$$

Next, we examine the EFE by considering a homogeneous and isotropically expanding background.

\subsection{The expanding universe}

Our homogeneous and isotopically expanding Universe is well described by the spatially flat Friedmann-Robertson-Walker (FRW) metric [24] given by

$$
\mathrm{d} s^{2}=g_{\mu \nu} \mathrm{d} x^{\mu} \mathrm{d} x^{\nu}=g_{00} \mathrm{~d} t^{2}+g_{i j} \mathrm{~d} x^{i} \mathrm{~d} x^{j},
$$

where

$$
g_{00}=-1 \quad \text { and } \quad g_{i j}=a^{2}(t) \delta_{i j}
$$

where $\delta_{i j}=1$ for $i=j$ and it is zero for $i \neq j$. The time-dependent coefficient $a(t)$ is the scale factor, which is taken to increase monotonically, and $x^{i}$ are the commoving coordinates related to the physical coordinates by the transformation $x^{i}=a(t) x^{i}$. Hence, the physical distance between commoving points is proportional to the cosmological factor $a(t)$, i.e. as time evolves the distance between commoving points gets larger and larger resulting to an expanding universe. Introducing conformal time

$$
\mathrm{d} \tau=\frac{\mathrm{d} t}{a(t)}
$$

the metric becomes

$$
g_{00}=-a^{2}(t) \quad \text { and } \quad g_{i j}=a^{2}(t) \delta_{i j} .
$$

In this way, the metric is factorized into the Minkowski metric times the timedependent conformal factor $a(\tau)$. Rescaling by $x^{i}=a(t) x^{i}$ reduces (1.2.4) back into the Minkowski one. Although the distance between comoving points is changing, their coordinates remain the same. 
Furthermore, in an expanding universe (assuming no other external forces apart of gravity) the momenta of particles are inversely proportional to the scale factor [29]. For massless particles that is $E=k_{p h} \propto a^{-1}$. In other words, the momenta $k$ and energy $E$ of free particles and fields decrease as the spacetime fabric expands, while their wavelength $\lambda_{p h} \propto a$ increases in proportion to the scale factor. We say that the proper wavelength of a photon emitted at some initial time $t_{i n}$ and observed at time $t_{0}$ is redshifted where the redshift $z$ is defined as

$$
(1+z)=\frac{\lambda_{0}}{\lambda_{i n}}=\frac{a\left(t_{0}\right)}{a\left(t_{i n}\right)}
$$

It is convenient to work with the FRW metric in spherical polar coordinates, given by

$$
g_{00}=-1, \quad g_{r r}=\frac{a^{2}(t)}{1-\mathcal{K} r^{2}}, \quad g_{\theta \theta}=a^{2}(t) r^{2} \quad \text { and } \quad g_{\phi \phi}=a^{2}(t) \sin ^{2} \theta r^{2}
$$

where $\mathcal{K}$ describes the curvature of the universe, which is positive if $\mathcal{K}=1$, negative if $\mathcal{K}=-1$ or zero if $\mathcal{K}=0$. The radial distance, from an event stationed at the centre of a sphere, is given by $r$.

The Friedmann equations (Appendix A.4) describe the dynamic evolution of the scale factor. The first Friedmann equation is given by

$$
H^{2}=\frac{8 \pi G}{3}\left(\rho+\frac{\Lambda}{8 \pi G}\right)-\frac{\mathcal{K}}{a^{2}},
$$

and the second Friedmann equation is given by

$$
\frac{\ddot{a}}{a}=-4 \pi G\left(p+\frac{\rho}{3}\right)+\frac{\Lambda}{3}
$$

where

$$
H(t)=\frac{\dot{a}(t)}{a(t)}=\frac{\mathcal{H}(\tau)}{a(\tau)},
$$

is the Hubble parameter and for positive values $H>0$ describes an expanding universe. The evolution of the energy density $\rho(t)$ is given by the fluid equation which can be easily derived by employing the first law of thermodynamics [30], giving

$$
\dot{\rho}+3 \frac{\dot{a}}{a}\left(\rho+\frac{p}{\rho}\right)=0 .
$$

We can find a relation for the scale factor at a particular epoch by solving the above equations for a known matter density $\rho$ and pressure $p$ [31]. Therefore, for a radiation dominated universe we have 


$$
\rho_{R} \propto a^{-4}, \quad \text { with } \quad a \propto t^{\frac{1}{2}} \propto \tau,
$$

and for a matter dominated universe

$$
\rho_{m} \propto a^{-3}, \quad \text { with } a \propto t^{\frac{2}{3}} \propto \tau^{2} .
$$

From this we conclude that the energy density of radiation (i.e. relativistic particles) decreases much faster, as the volume of the universe expands, due to that its wavelength is redshifted. While the energy density of matter goes as inversely proportional to the volume (i.e. $\rho_{m} \propto a^{-3}=V^{-1}$ ). The critical density $\rho_{c}$ is the density required for the geometry of our present universe to be flat and can be computed from (1.2.7) by setting $\mathcal{K}=0$ and $\Lambda=0$, giving

$$
\rho_{\text {crit }}(t)=\frac{3}{8 \pi G} H^{2}(t)
$$

with the current value being $\rho_{c}\left(t_{0}\right) \sim 10^{-29} \mathrm{~g} / \mathrm{cm}^{3}$ (this is about 6 hydrogen atoms per cubic meter) [32]. We can re-express (1.2.7) in terms of the density parameter, defined as the ratio of the density of matter and energy to the critical density

$$
\Omega=\frac{\rho(t)}{\rho_{\text {crit }}(t)}=\frac{8 \pi G \rho}{3 H^{2}},
$$

which describes the density of matter and energy in the universe at any time. From this we get

$$
\Omega-1=\frac{\mathcal{K}}{a^{2}(t) H^{2}(t)},
$$

where we divided everything by $H^{2}$ and absorbed the cosmological constant into the definition for $\Omega$. Using (1.2.11) and (1.2.12), to calculate the value of the scale factor at different epochs, we can see that in the standard Big Bang model the quantity on the RHS of (1.2.15) always increases with time, i.e. we find that $\Omega-1 \sim t^{\frac{2}{3}}$ during radiation era and $\Omega-1 \sim t$ during matter era. Therefore, the value of $\Omega$ would have been much larger than unity in the early universe (i.e. $\Omega \sim 10^{-60}$ ) [30]. This is the so called flatness problem, in the standard model of cosmology, as it requires extremely fine tuned initial conditions in order to recover the observed value of the spatial curvature of the universe, which is flat $\left(\Omega_{k}=0.001 \pm 0.002\right)$ [33]. With inflation the initial value of $\Omega$ does not play any significant role anymore. The scale factor increases for such an enormous amount (say $10^{27}$ or a lot more) that any curvature in the geometry of spacetime becomes approximately flat at the end of inflation. This is easy to see, as the denominator in the RHS of (1.2.15) will quickly attain a value $(a H)^{2} \gg 1$ therefore, $\Omega-1 \sim 0$ and we can safely consider the universe to be flat with $\mathcal{K}=0$ at the time of decoupling and to, consequently, remain this way till the present day.

Next, we look at the horizon problem. The value of $H^{-1}$ defines the horizon size at each epoch ${ }^{1}$, that is, the maximum distance at which an observer, stationed

\footnotetext{
${ }^{1}$ With our conventions $t \sim H^{-1}$ is the Hubble time and $d \sim H^{-1}$ is the Hubble length.
} 
at the centre of a sphere with radius $r$, would be able to receive light signals from. As light signals travel in null geodesics ${ }^{2}$, then the line element in (1.2.1) becomes $\mathrm{d} s^{2}=0=-\mathrm{d} t^{2}+a^{2}(t) \mathrm{d} r^{2}$. Integrating the quantity $\mathrm{d} t a(t)^{-1}$, over a finite time interval, gives the comoving distance travelled by the signal, which reads

$$
\int \mathrm{d} r=\tau=\int_{0}^{t} \frac{\mathrm{d} t^{\prime}}{a\left(t^{\prime}\right)}
$$

Assuming the early universe was matter and radiation dominated, we can use relations (1.2.11) and (1.2.12) to estimate the size of patches in the sky in which physics would have been in causal contact some time in the past. Extrapolating this to the present time $\tau_{0}$ one finds that physics would be correlated only for points on the CMB sky that are $1^{\circ}$ apart [34]. This does not explain why we see a homogeneous temperature in the $\mathrm{CMB}$ for points that are, for example, $180^{\circ}$ apart in the sky. This is known as the horizon problem. Next, we look at how inflation solves this problem.

As the Friedmann equations have three unknowns $a(t), p(t)$ and $\rho(t)$, we need one more relation to help us relate these parameters. Assuming a homogeneous and isotropic mass-energy distribution, the material content of our system is characterized by a constant equation of state

$$
p=\omega \rho,
$$

where $\omega$ is a dimensionless number. We can write the acceleration equation in $(1.2 .8)$, as

$$
\frac{\ddot{a}}{a}=-(1+3 \omega)\left(\frac{4 \pi G}{3} \rho\right) .
$$

For positive acceleration $\ddot{a}>0$ it requires that $1+3 \omega<0$, meaning that the equation of state is characterized by negative pressure $p<0$. Therefore, $\omega<-1 / 3$ denotes a universe with accelerated expansion [35].

Let us look at the case where matter is taken to be negligible $\left(\rho_{m} \simeq p_{m} \simeq 0\right)$. The Friedmann equation in (1.2.7) reduces to

$$
H^{2}=\frac{8 \pi G}{3} \rho_{\Lambda},
$$

which results to the scale factor growing exponentially with time $a(t) \propto e^{H t}$. This is known as the de Sitter spacetime which will be the focus of this work. Using relationship (1.2.3) for the conformal time we find that the definition of the scale factor in de Sitter space is

$$
\tau=-\frac{1}{a H}
$$

The usual convention is that the conformal time is negative during the inflationary period, where $\tau \rightarrow 0$ denotes the end of the inflationary period, while $\tau \rightarrow \infty$ denotes the infinite past.

\footnotetext{
${ }^{2}$ In this case two points are linked by a radial path and $\mathrm{d} \theta=0, \mathrm{~d} \phi=0$.
} 
In the theory of inflation we postulate a period of rapid expansion of space time where the scale factor grew by a factor of $e$ per Hubble time which is denoted by an $e$ folding [31]. This remedies the so called horizon problem, as during inflation physical scales grow proportionally with the scale factor $\lambda_{p h} \sim a \sim \exp (H t)$. Therefore, at some point in the far past they must have been within the horizon $\lambda_{p h}<H^{-1}$ and in causal contact. After that the universe must had gone through a period of rapid expansion where the length scales $\lambda$ grew faster than the horizon scale. This way regions of spacetime that would seem to be causally disconnected in the Big Bang model of cosmology, can come into causal contact and therefore into thermal equilibrium, in agreement with predictions. Next we take a closer look at the theory of inflation.

\subsection{Inflation from a single scalar field}

In slow-roll inflation we assume that, in the first moments after the Big Bang, a scalar field dominated the energy content of the universe. The potential is taken to be almost flat so that the scalar field evolves slowly and that its behaviour effectively resembles that of a cosmological constant as seen in Figure (1.2)

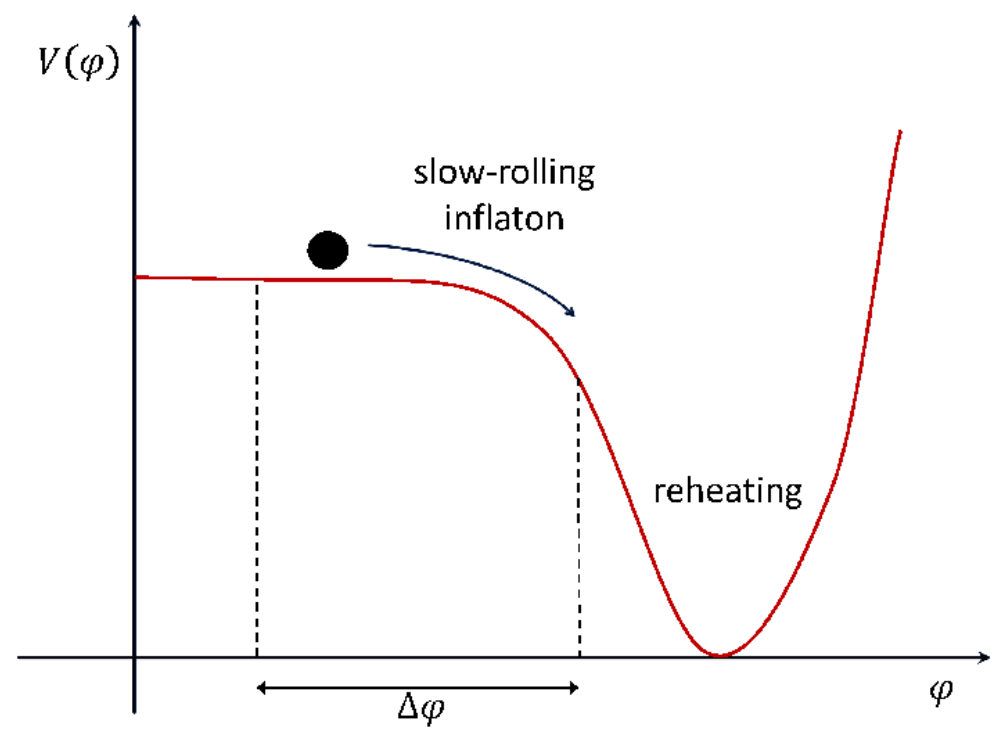

Figure 1.2: Inflaton rolling down its potential [1]. The amount of inflation is controlled by the "flatness" of the potential. The excursion of the field between horizon exit and the end of inflation is denoted by $\Delta \phi$.

Slow-roll inflation ends when the scalar fields reaches the bottom of the potential, which is where the scalar field starts to oscillate around its minimum and it consequently decays into standard model particles. This process is called reheating. The process described so far is model-dependent and it heavily depends on the shape of the potential. In Figure (1.3) we see the latest constraints from the Planck Collaboration on a selection of inflaton potentials. 


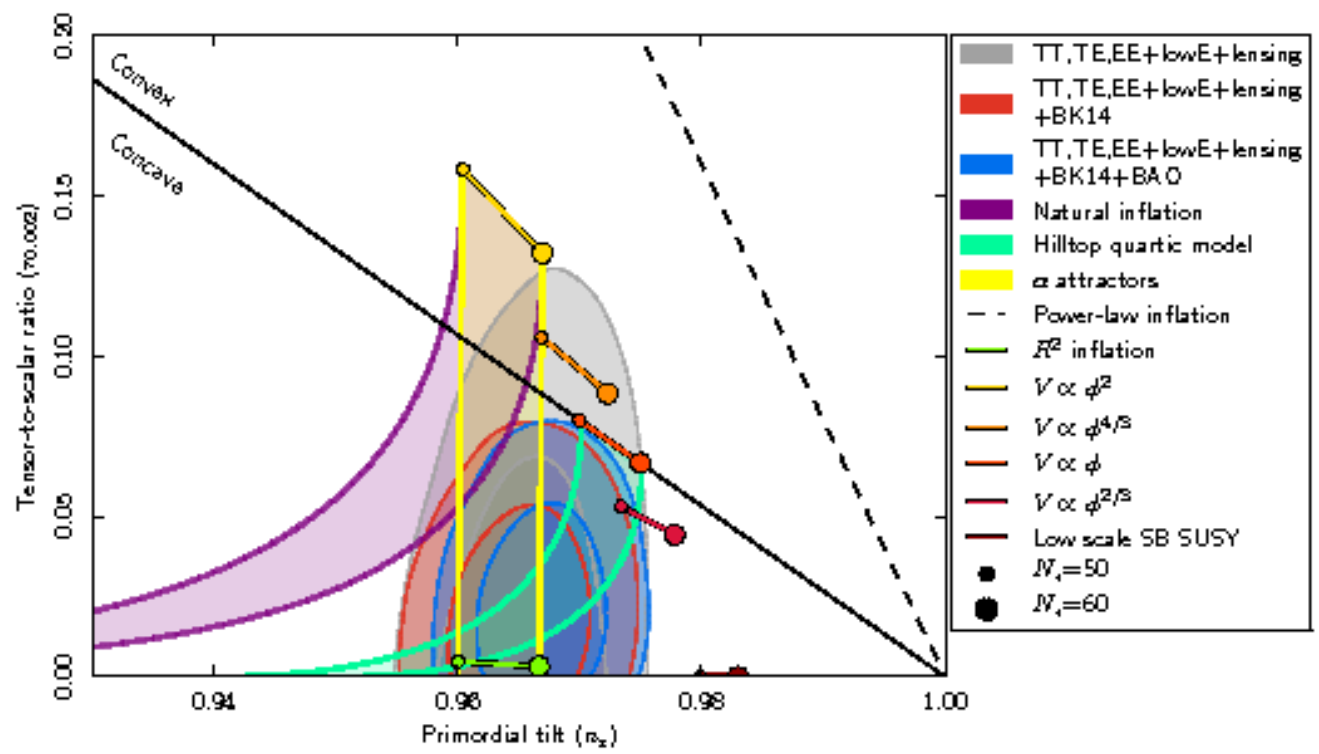

Figure 1.3: A comparison to theoretical predictions for inflationary models with data from Planck alone and in combination with BK14 or BK14 plus BAO data, for $r$ and $n_{s}$ at the pivot scale $k=0.002 / M p c^{-1}$ [2].

There are many models that can lead to inflation. Here we consider the case where the dynamics of inflation are governed by a single scalar field $\phi$, known as the inflaton. In this case the action is given by (1.1.21), where the scalar field is taken to be minimally coupled to the metric via the canonical kinetic term.

More general models can involve non-minimal couplings between the scalar field $\phi$ and the curvature $R$, generalizations of the gravitational sector by including functions of the form $f(R)[18]$ or non-canonical kinetic terms [36]. In this work we focus on the latter. In particular, in this work we are interested to study the dynamics of the tensor sector therefore, we do not review inflationary models. For a pedagogical introduction to inflationary models see [21,37].

Let us start by considering the Lagrangian for a single scalar field with canonical kinetic term and a potential defined as in (1.1.21). We assume an isotropic and homogeneous FRW cosmology with line element

$$
\mathrm{d} s^{2}=-\mathrm{d} t^{2}+a^{2}(t) \mathrm{d} x^{2} .
$$

For a homogeneous scalar field $\phi=\phi(t)$ in FRW spacetime $\left(\sqrt{-g}=a^{3}\right)$, the EulerLagrange equations (1.1.12) give (Appendix A.5)

$$
\ddot{\phi}+3 H \dot{\phi}+\frac{\partial U(\phi)}{\partial \phi}=0
$$

where an overdot indicates a derivative with respect to time $t$ and the Hubble parameter is defined by $H=\dot{a} / a$. For now, $\phi$ is taken to be a classical scalar field. We will look at quantum fluctuations later in this section. 
The second term in (1.3.2) is understood as a friction term which damps the velocity of the field due to the expansion of the universe. Furthermore, the Friedmann equations become

$$
H^{2}=\frac{8 \pi G}{3}\left[\frac{1}{2} \dot{\phi}^{2}+U(\phi)\right]
$$

The EMT in (1.1.22) for an perfect fluid becomes

$$
\begin{aligned}
& \rho=\frac{1}{2} \dot{\phi}^{2}+U(\phi), \\
& p=\frac{1}{2} \dot{\phi}^{2}-U(\phi) .
\end{aligned}
$$

From this, the equation of state for a homogeneous scalar field becomes

$$
\omega=\frac{p}{\rho}=\frac{\frac{1}{2} \dot{\phi}^{2}-U(\phi)}{\frac{1}{2} \dot{\phi}^{2}+U(\phi)},
$$

and we find that the de Sitter condition $p=-\rho$ is satisfied for as long as the kinetic energy is negligible compare to the potential energy $\dot{\phi}^{2} \ll U(\phi)$. From this we see that the potential energy dominates and acts like a cosmological constant, i.e. $\omega \rightarrow-1$, which leads to the accelerated expansion of the Universe. With these considerations, the Friedmann equations (1.3.3) reduce to

$$
H^{2}=\frac{8 \pi G}{3} U(\phi)
$$

To ensure that this constraint is satisfied we also demand that $\ddot{\phi} \ll \partial U / \partial \phi$. Assuming that the friction term dominates we have that (1.3.2) becomes

$$
3 H \dot{\phi}+\frac{\partial U(\phi)}{\partial \phi}=0 \Rightarrow \dot{\phi}=-\frac{1}{3 H} \frac{\partial U(\phi)}{\partial \phi} .
$$

Equations (1.3.6) and (1.3.7) are known as the slow-roll approximations. Next, it is useful to introduce the slow-roll parameters $\epsilon$ and $\eta$. From the second Friedmann equation we find that for the potential to be sufficiently flat, it requires

$$
\epsilon=-\frac{\dot{H}}{H^{2}} \ll 1 \quad \text { and } \quad \eta=-\frac{\ddot{\phi}}{H \dot{\phi}}=-\frac{1}{2} \frac{\ddot{H}}{\dot{H} H} \ll 1 .
$$

The parameter $\epsilon$ is used to describe how much the Hubble parameter differs from a constant. The condition for inflation demands that this parameter is very small so that the condition for accelerated expansion $\ddot{a} \gg 0$ is satisfied. The parameter $\eta$ is 
just the requirement that $\ddot{\phi} \ll \partial U / \partial \phi$. The parameter $\epsilon$ can be expressed in terms of the number of $e$-folds, which is a way of measuring how the scale factor increases. In this way we can measure the amount of inflation. We can do that by making use of that the Hubble parameter can be expressed as $H t=\ln a \Rightarrow \mathrm{d} N=\mathrm{d} \ln a=-H \mathrm{~d} t$, where we adopt this sign convention so that as the scale factor increases $N$ decreases, i.e. $a \propto \exp \int(H \mathrm{~d} t)=\exp (-N)$. Assuming $\Omega-1 \sim 10^{-60}$, inflation can ensure $\Omega_{0} \sim 1$ if $N \sim 70$ e-folds [38]. For $\epsilon \rightarrow 0$ we find the de Sitter limit $p=-\rho$. In the main text of this work we focus on de Sitter space with $H=$ const.

The inhomogeneities we observe today, in the matter content of the Universe, are thought to have started as quantum fluctuations that were, consequently, stretched by inflation and became classical. These are expressed as perturbations around the inflationary background. The scalar and metric fields can be expanded as

$$
\phi(t, \mathbf{x})=\bar{\phi}(t)+\delta \phi(t, \mathbf{x}) \quad \text { and } \quad g_{\mu \nu}(t, \mathbf{x})=\bar{g}_{\mu \nu}+h_{\mu \nu}(t, \mathbf{x}) .
$$

These fluctuations are treated as small variations away from the homogeneous and isotropic background. Therefore at the level of the fluctuations the universe is not necessarily homogeneous and/or isotropic which means that the geometry where the fluctuations live is different than that of the background. Consequently, in order to study these fluctuations we need to choose a specific spacetime gauge i.e. a map that links points between the two geometries.

The usual procedure is to first foliate spacetime into slices of spacelike surfaces of constant time $t$ and thread it in terms of timelike worldlines of constant $\mathbf{x}$. In the case of FRW spacetime, comoving observers see a uniform energy density (i.e. $\rho(t)=$ const). A change of coordinates can introduce gauge artefacts, i.e. inhomogeneities in spacetime that are not real but a result of changing coordinates. For this reason it is important to define gauge-invariant quantities.

The metric can be decomposed, according to its symmetries, into Scalar, Vector and Tensor components via the SVT decomposition [39]. At linear order these are known to decouple and can be treated separately. In terms of these we can define the gauge-invariant quantity known as the comoving curvature perturbation $\mathcal{R}$. Assuming an ADM decomposition of spacetime [93], i.e. where the spacetime is sliced into three-dimensional hypersurfaces of constant time $t$, we can geometrically relate the comoving curvature perturbations $\mathcal{R}$ to the spatial curvature by $R^{(3)}=$ $4 \nabla^{2} \mathcal{R} / a^{2}$.

During a time of quasi-de Sitter expansion, time-diffeomorphisms are broken due to the homogeneous but time-dependent background. It is typical to treat the perturbations in a similar manner and consider dynamics that break timediffeomorphism invariance but preserve spatial diffeomorphisms, i.e. under timediffeomorphism the scalar field fluctuations transform as

$$
\delta \phi \rightarrow \delta \phi-\dot{\phi} \xi_{0}
$$

It is convenient to work in the comoving gauge, which is defined as [40] 


$$
\delta \phi=0, \quad g_{i j}=a^{2}\left[(1-2 \mathcal{R}) \delta_{i j}+h_{i j}\right]
$$

where the tensors $h_{i j}$ are the transverse traceless perturbations of the metric, which we will review later, and $\mathcal{R}$ is the comoving curvature perturbation. This is a useful quantity because it stays constant for modes that cross the horizon, which means that we can compute correlations at horizon crossing, as we shall soon see. In this way all dynamical degrees of freedom are contained in the metric, i.e. we treat the scalar field as unperturbed. This makes it explicit that in this scenario inflation is driven by a single scalar field (the scalar degree of freedom contained in the metric) and we can use its background value as a clock for inflation.

Through this procedure, the quadratic action for the curvature perturbations becomes $\left(M_{P l}=1\right)$

$$
S_{\mathcal{R}}^{(2)}=\frac{1}{2} \int \mathrm{d}^{4} x a^{3} \frac{\dot{\phi}^{2}}{H^{2}}\left[(\dot{\mathcal{R}})^{2}-\frac{(\nabla \mathcal{R})^{2}}{a^{2}}\right] .
$$

This can be brought into the canonical form by defining the canonically normalized scalar field $v=z \mathcal{R}$, giving

$$
S_{\mathcal{R}}^{(2)}=\frac{1}{2} \int \mathrm{d} \eta \mathrm{d}^{3} x\left[\left(v^{\prime}\right)^{2}+(\nabla v)^{2}+\frac{z^{\prime \prime}}{z} v^{2}\right]
$$

where prime denotes derivatives with respect to the conformal time $\eta$ and the potential is given in terms of

$$
z=a \frac{\dot{\phi}}{H}
$$

In Fourier space we define

$$
v(\mathbf{x}, \eta)=\frac{1}{(2 \pi)^{3}} \int \mathrm{d}^{3} k v(\mathbf{k}, \eta) e^{i \mathbf{k} \cdot \mathbf{x}},
$$

from which we get the Euler-Lagrange equations in Fourier space, to be

$$
v(\mathbf{k}, \eta)^{\prime \prime}+\left(k^{2}-\frac{z^{\prime \prime}}{z}\right) v(\mathbf{k}, \eta)=0
$$

The quantization of the field proceeds as in the standard case for scalar fields in curved spacetime [41]. The field $v$ and its conjugate momentum

$$
\pi=\frac{\partial \mathcal{L}}{\partial\left(\partial_{0} v\right)}=\partial_{\eta} v
$$


are promoted into quantum operators. Next we expand it in terms of positive and negative frequency modes, as

$$
v(\mathbf{x}, \eta)=\frac{1}{(2 \pi)^{3}} \int \mathrm{d}^{3} k\left[v(\mathbf{k}, \eta) \hat{a}(\mathbf{k}) e^{i \mathbf{k} \cdot \mathbf{x}}+v^{*}(\mathbf{k}, \eta) \hat{a}^{\dagger}(\mathbf{k}) e^{-i \mathbf{k} \cdot \mathbf{x}}\right],
$$

where the raising and lowering operators obey the following commutation relations

$$
\left[\hat{a}(\mathbf{k}), \hat{a}^{\dagger}\left(\mathbf{k}^{\prime}\right)\right]=(2 \pi)^{3} \delta\left(\mathbf{k}-\mathbf{k}^{\prime}\right)
$$

and where $a|0\rangle$ annihilates the Bunch-Davies vacuum ${ }^{3}$. Here, the normalization of the mode functions is expressed in terms of the the Wroskian, which is the conserved quantity

$$
W\left(u(\mathbf{k}, \eta), u^{*}(\mathbf{k}, \eta)\right)=u^{*}(\mathbf{k}, \eta) \partial_{\eta} u(\mathbf{k}, \eta)-u(\mathbf{k}, \eta) \partial_{\eta} u^{*}(\mathbf{k}, \eta)=i .
$$

The derivation of the above involves taking the sub-horizon limit $|k \eta| \gg 1$ in (1.3.16), giving the equation of a simple harmonic oscillator

$$
v(\mathbf{k}, \eta)^{\prime \prime}+k^{2} v(\mathbf{k}, \eta)=0 .
$$

We fix the initial condition by choosing the positive frequency mode

$$
\lim _{|k \eta| \rightarrow \infty} v(\mathbf{k}, \eta)=\frac{1}{\sqrt{2 k}} e^{-i k \eta}
$$

which translates to that the fluctuations deep within the horizon live in the BunchDavies vacuum which defines the minimum energy state.

To derive (1.3.20) we need to multiply (1.3.21) by $u *$ as well multiply the conjugate of (1.3.21) by $u$ and subtract the second from the first, giving $u^{*} u^{\prime \prime}-u u^{* \prime \prime}=0$. Then, using the product rule gives $\partial_{\eta}\left(u^{*} u^{\prime}-u u^{* \prime}\right)=0$ implying that the term inside the brackets, which we identify with the Wroskian, must be a constant. To find its value one simply needs to substitute in terms of the expressions for the mode functions $v$ and $v^{*}$ which are expressed in terms of plane wave solutions, giving the result in (1.3.20).

Assuming a de Sitter spacetime ( $H=$ const), one can find an exact solution to (1.3.16), given by

$$
u(\mathbf{k}, \eta)=\frac{1}{\sqrt{2 k}}\left[b_{1}\left(1-\frac{i}{k \eta}\right) e^{-i k \eta}+b_{2}\left(1+\frac{i}{k \eta}\right) e^{i k \eta}\right] .
$$

\footnotetext{
${ }^{3}$ For $|k \eta| \rightarrow \infty$ this coincides with the Minkowski vacuum.
} 
We can use the conditions (1.3.20) and (1.3.22) to fix the coefficients $b_{1}=1$ and $b_{2}=0$, which leads to the unique solution

$$
u(\mathbf{k}, \eta)=\frac{1}{\sqrt{2 k}}\left(1-\frac{i}{k \eta}\right) e^{-i k \eta}
$$

The scalar curvature power spectrum is defined in terms of

$$
\langle\mathcal{R}(\mathbf{k}, \eta) \mathcal{R}(\mathbf{q}, \eta)\rangle=(2 \pi)^{3} \delta^{(3)}(\mathbf{k}+\mathbf{q}) P_{\mathcal{R}}(k),
$$

where $P_{\mathcal{R}}$ is the dimensionless power spectrum, given by

$$
P_{\mathcal{R}}(k) \equiv \frac{k^{3}}{2 \pi^{2}} \frac{|u(\mathbf{k}, \eta)|^{2}}{|z(\mathbf{k}, \eta)|^{2}},
$$

where $z=a$ in de Sitter. Taking the superhorizon limit of the solutions, i.e. $\lim _{|k \eta| \rightarrow 0} u(k, \eta)$, we find

$$
P_{\mathcal{R}}(k)=\frac{H^{2}}{(2 \pi)^{2}} \frac{H^{2}}{\dot{\phi}^{2}},
$$

which is evaluated at horizon crossing $a H=k$. So far, data suggests that the density perturbations from inflation at large scales are highly adiabatic [2]. We view adiabatic fluctuations from inflation as shifts along the trajectory of the homogeneous background field in the forward and backward direction. Shifts in other directions would result to non-adiabatic density perturbations. In terms of the radiation and matter content in the Universe, we find $\delta\left(n_{m} / n_{r}\right) \simeq 0$, meaning that all density perturbations are a result of the same curvature perturbation $\mathcal{R}$ and not the result of any other type of perturbations, such as isocurvature modes which are orthogonal to the background trajectory and are usually present in multi-field models of inflation [35]. 


\section{Introduction to primordial grav- itational waves}

We have seen that although the CMB is mostly homogeneous, it still contains inhomogeneities of the form $\delta \rho / \rho \sim 10^{-5}$. We believe these to be the seeds that grew to form their own self-gravitating systems which led to the structure formation we see in the Universe today.

In this section we are interested to understand where these inhomogeneities may have come from. We believe them to be quantum fluctuations of the inflaton field which, during inflation, grew exponentially and exited the horizon, which is where their amplitudes freeze, to then re-enter the horizon once inflation ended and the Hubble radius started to grow. Through the Einstein equations we find that metric perturbations, during inflation, were also stretched to cosmological scales. This can be understood as perturbations in the scalar sector $\delta \phi$ imply perturbations in the energy momentum tensor $\delta T_{\mu \nu}$ which, through the EFE, imply perturbations in the metric $\delta g_{\mu \nu}$. Consequently the metric perturbations induce a back-reaction on the scalar field perturbations due to that the later are minimally coupled to gravity. Therefore, we study the evolution of perturbations around a homogeneous and isotropic FRW background

$$
\phi(t, \mathbf{x})=\bar{\phi}(t)+\phi^{(1)}(t, \mathbf{x}) \quad \text { and } \quad g_{\mu \nu}(t, \mathbf{x})=\bar{g}_{\mu \nu}(t)+g_{\mu \nu}^{(1)}(t, \mathbf{x}),
$$

where the line element for a perturbed metric is given by

$$
\mathrm{d} s^{2}=\left[\bar{g}_{\mu \nu}(t)+g_{\mu \nu}^{(1)}(t, \mathbf{x})\right] \mathrm{d} x^{\mu} \mathrm{d} x^{\nu} .
$$

Here, $\bar{\phi}$ is a background scalar field and $\phi^{(1)}$ is a small fluctuation of the scalar field $\left(\phi^{(1)} \ll \bar{\phi}\right)$. The background metric is denoted by $\bar{g}_{\mu \nu}$, while $g_{\mu \nu}^{(1)}$ describe the linear perturbations of the metric $\left(g_{\mu \nu}^{(1)} \ll \bar{g}_{\mu \nu}\right)$. The metric perturbations can be decomposed into scalar, vector and tensor perturbations known as the (STV) decomposition, which is how they are categorized according to their symmetries in the $3+1$ decomposition of space time i.e. how they transform under rotations on the three-dimensional spatial hypersurface. Scalar perturbations have spin 0 and lead to inhomogeneities responsible for structure formation, vector perturbations have spin 1 and are known to decay as $1 / a(t)$ during inflation, while tensor perturbations 
have spin 2 and give rise to primordial gravitational waves. At linear order the scalar, vector and tensor perturbations are known to decouple, so it is customary to consider their evolution independently from one another [40]. In this work we are interested on tensor degrees of freedom therefore, we won't spend much time in the analysis of scalar degrees of freedom. In particular, in this work we are interested on primordial gravitational waves (tensor perturbations) which are ripples in space and time that were generated during inflation. We review the subject of gravitational waves next.

\subsection{Linearised gravity}

Gravitational waves are understood as deformations on the background spacetime. In linearised gravity, one considers perturbations around a flat metric $(a(t)=1)$, of the form

$$
g_{\mu \nu}=\eta_{\mu \nu}+h_{\mu \nu}, \quad\left|h_{\mu \nu}\right| \ll 1 .
$$

We use the background metric $\eta_{\mu \nu}=\operatorname{diag}(-1,1,1,1)$ to rise and lower indices of the metric perturbations $h_{\mu \nu}$. The symmetric tensor $h_{\mu \nu}$ is a solution to the linearised Einstein field equations and it is treated as a plane wave where the direction of propagation is taken to be the longitudinal one, usually the $z$-direction, while the directions perpendicular to it are called transverse. We can obtain the linearised Einstein equations by substituting for (2.1.1) in

$$
G_{\mu \nu}^{(1)}=R_{\mu \nu}^{(1)}-\frac{1}{2} R^{(1)} \eta_{\mu \nu}=8 \pi G T_{\mu \nu}
$$

Using relations (1.1.3), (1.1.6) and (1.1.7), it is straightforward to show that

$$
R_{\mu \nu}^{(1)}=\frac{1}{2}\left(-\square h_{\mu \nu}+\partial_{\alpha} \partial_{\nu} h_{\mu}^{\alpha}+\partial_{\mu} \partial_{\alpha} h_{\nu}^{\alpha}-\partial_{\mu} \partial_{\nu} h\right)
$$

and

$$
R^{(1)}=\partial_{\mu} \partial_{\nu} h^{\mu \nu}-\square h,
$$

where $h=h_{\mu}^{\mu}$ and $\square=\partial_{\mu} \partial^{\mu}=-\partial_{t}^{2}+\nabla^{2}$. Under a coordinate (gauge) transformation, of the form

$$
x^{\prime \mu}=x^{\mu}+\xi^{\mu}(x),
$$

where $\xi^{\mu}(x)$ are treated as four arbitrary functions, the metric perturbation transforms as 


$$
h_{\mu \nu}^{\prime} \rightarrow h_{\mu \nu}-\partial_{\mu} \xi_{\nu}-\partial_{\nu} \xi_{\mu}, \quad\left|\partial_{\mu} \xi_{\nu}(x)\right| \ll 1
$$

To simplify the EFE we impose the Lorentz gauge condition

$$
\partial^{\mu} \bar{h}_{\mu \nu}=0
$$

where we defined the trace reversed perturbation

$$
\bar{h}_{\mu \nu}=h_{\mu \nu}-\frac{h}{2} \eta_{\mu \nu}, \quad \bar{h}=-h .
$$

This transforms as

$$
\bar{h}_{\mu \nu}^{\prime} \rightarrow h_{\mu \nu}-\partial_{\mu} \xi_{\nu}-\partial_{\nu} \xi_{\mu}+\eta_{\mu \nu} \partial_{\rho} \xi^{\rho}, \quad\left(\partial^{\nu} \bar{h}_{\mu \nu}\right)^{\prime}=\partial^{\nu} \bar{h}_{\mu \nu}-\square \xi_{\mu}
$$

From the above we see that the Lorentz gauge holds if

$$
\partial^{\nu} \bar{h}_{\mu \nu}=\square \xi_{\mu}=0
$$

This can be used to simplify the expressions for (2.1.3) and (2.1.4), so that (2.1.2) becomes

$$
\square \bar{h}_{\mu \nu}=-16 \pi G T_{\mu \nu},
$$

where $\partial^{\nu} T_{\mu \nu}=0$. The condition in (2.1.7) reduces the number of independent components of the symmetric tensor $h_{\mu \nu}$ to six. Away from the source, we have that $T_{\mu \nu}=0$, so that this equation simplifies even further to

$$
\square \bar{h}_{\mu \nu}=0
$$

These are the EFE in vacuum, where metric perturbations are taken to propagate with the speed of light $(c=1)$ and have plane wave solutions. Next we remove another four degrees of freedom by using the so called transverse-traceless gauge (TT). Looking at (2.1.7), we are still allowed a transformation of the form (2.1.5) with (2.1.10) and thefore, $\square \xi_{\mu \nu}=0$. We can use the residual gauge freedom in (2.1.10) to choose the arbitrary functions $\xi_{\mu}$ so that $\bar{h}=0, \bar{h}_{\mu \nu}=h_{\mu \nu}$ and all four components $h_{0 \mu}=0$. This way the TT gauge reads

$$
h^{0 \mu}=h_{i}^{i}=\partial^{j} h_{i j}=0 .
$$


This condition only holds away from the source. From this we see that metric fluctuations are purely spatial and transverse with respect to the direction of propagation of the gravitational wave as well trace free. The general solutions to equation (2.1.12) are plane waves

$$
h_{\mu \nu}(t, x)=P_{\mu \nu} e^{i k_{\alpha} x^{\alpha}}+P_{\mu \nu}^{*} e^{-i k_{\alpha} x^{\alpha}},
$$

where the amplitude and phase of the gravitational waves is described by the polarization tensor $P_{\mu \nu}$ and $k_{\alpha}=(\omega, \mathbf{k})$ is a wavevector. Substituting the solution (2.1.14) into the wave equation in (2.1.12), we find that $k^{2}=k_{\mu} k^{\mu}=-\omega^{2}+\mathbf{k}^{2}=0$ is a null vector, where the frequency of the wave is given by $\omega=|\mathbf{k}|$. Therefore, gravitational waves, in the standard theory of General Relativity, propagate with the speed of light $(c=1)$. For a given direction of propagation $\mathbf{n}=\mathbf{k} /|\mathbf{k}|$ the nontrivial components of the symmetric and traceless tensor $h_{i j}$ will be on the transverse directions to $\mathbf{n}$. Then, the amplitude for a gravitational wave travelling along the $z$-direction can be written as a linear combination of the two polarization tensors

$$
P_{i j}=\left(h^{+} p_{i j}^{+}+h^{\times} p_{i j}^{\times}\right),
$$

where $p_{i j}$ are the two linear polarization tensors and are defined as

$$
p_{i j}^{+}=\left(\begin{array}{cccc}
0 & 1 & 0 & 0 \\
0 & 0 & -1 & 0 \\
0 & 0 & 0 & 0
\end{array}\right), \quad p_{i j}^{\times}=\left(\begin{array}{cccc}
0 & 0 & 1 & 0 \\
0 & 1 & 0 & 0 \\
0 & 0 & 0 & 0
\end{array}\right)
$$

The plus and cross polarizations are at $45^{\circ}$ to each other. If we consider a particle at rest with $u^{\mu}=\left(u^{t}, 0,0,0\right)$, the geodesic equation vanishes $\left(\Gamma_{\mu \nu}^{\alpha}=0\right)$ which translates to that the particle is at free fall along the geodesic. In this sense, the coordinates are comoving with the particle. We can envision the passing of a gravitational wave as a deformation on a ring of free falling particles. The displacement $\Delta s^{2}$ can be calculated by perturbing the line element and using (2.1.14). In Figure (2.1) we visualize gravitational waves by looking at the motion of a ring of test particles. We see that as gravitational waves are transverse, they distort spacetime perpendicularly to their direction of propagation.

\subsection{Tensor polarizations}

Let the unit vectors $\mathbf{e}^{(1)}$ and $\mathbf{e}^{(2)}$ be orthogonal to each other and to the vector $\mathbf{k}=k^{i}$. To describe metric perturbations in a more general way that doesn't rely on choosing a particular set of basis vectors, we construct the matrices $p_{i j}^{s}(\mathbf{k})$, with $s=\times,+$, from components of the unit vectors $\mathbf{e}_{i}^{(1)}=\left(e_{1}^{(1)}, e_{2}^{(1)}, e_{3}^{(1)}\right)$, and $\mathbf{e}_{i}^{(2)}=\left(e_{1}^{(2)}, e_{2}^{(2)}, e_{3}^{(2)}\right)$. The plus and cross polarization tensors can then be expressed as 


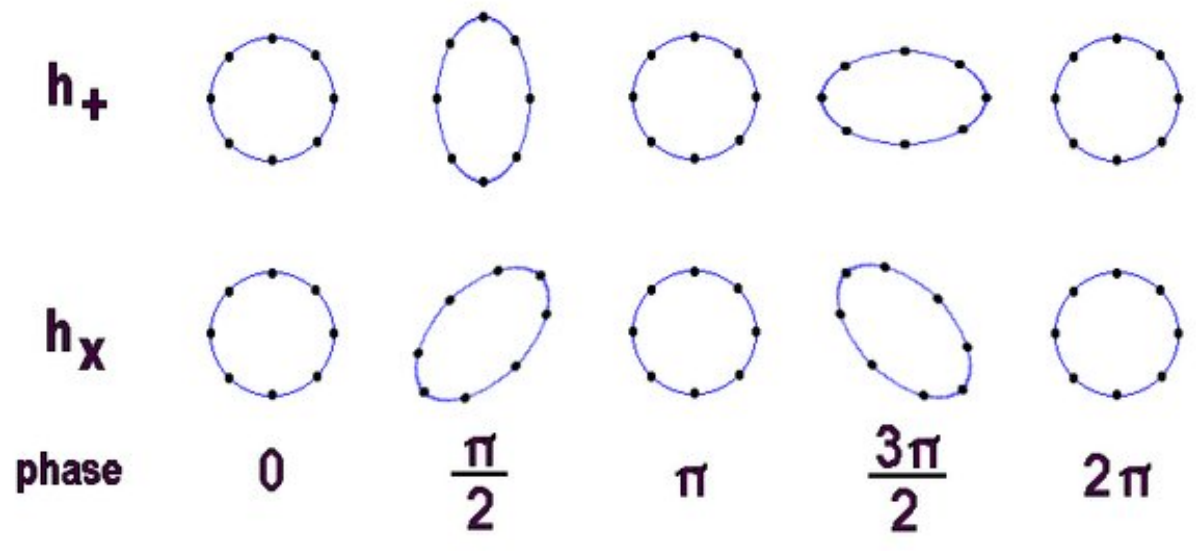

Figure 2.1: Distortion of a ring of particles by a gravitational wave travelling perpendicularly to the plane of the page. The image depicts the two polarizations of gravitational waves: plus (top) and cross (bottom). Image Credit: Wm. Robert Johnston, UT Dallas.

$$
p_{i j}^{+}(\mathbf{k})=\mathbf{e}_{i}^{(1)} \mathbf{e}_{j}^{(1)}-\mathbf{e}_{i}^{(2)} \mathbf{e}_{j}^{(2)} \quad \text { and } \quad p_{i j}^{\times}(\mathbf{k})=\mathbf{e}_{i}^{(1)} \mathbf{e}_{j}^{(2)}+\mathbf{e}_{i}^{(2)} \mathbf{e}_{j}^{(1)} .
$$

For example, one can reproduce the polarization matrices in (2.1.16) for $\mathbf{e}_{i}^{(1)}=$ $(1,0,0)$ and $\mathbf{e}_{i}^{(2)}=(0,1,0)$. Similarly, we can define the basis vectors in polar coordinates, as $\mathbf{e}_{i}^{(1)}=(\sin \phi,-\cos \phi, 0)$ and $\mathbf{e}_{i}^{(2)}=(\cos \theta \cos \phi, \cos \theta \sin \phi,-\sin \theta)$ for $\theta<\pi / 2$, where $\hat{\mathbf{n}}=\mathbf{k} /|\mathbf{k}|=(\sin \theta \cos \phi, \sin \theta \sin \phi, \cos \theta)$. Finally, under the transformation $\mathbf{k} \rightarrow-\mathbf{k}$ we have $\mathbf{e}_{i}^{(1)} \rightarrow-\mathbf{e}_{i}^{(1)}$ and $\mathbf{e}_{i}^{(2)} \rightarrow-\mathbf{e}_{i}^{(2)}$, i.e. $(\theta, \phi) \rightarrow$ $(\pi-\theta, \phi+\pi)$. Using (2.2.1) the polarization tensors are normalized as

$$
p_{i j}^{s}(\mathbf{k}) p_{i j}^{s^{\prime}}(\mathbf{k})=2 \delta^{s s^{\prime}}, \quad p_{i j}^{s}(\mathbf{k}) p_{i j}^{s}(\mathbf{k})=2,
$$

where we also have that $p_{i j}^{s}(\mathbf{k})=p_{i j}^{s}(-\mathbf{k})$. The plus and cross polarizations are orthogonal to the momenta (transversality/orthogonality condition) and traceless

$$
p_{i j}^{s} k_{j}=0 \quad \text { and } \quad p_{i}^{i s}=0 .
$$

Also, using the above properties and that the perturbations are real $h_{i j}^{*}=h_{i j}$, we have that $h_{i j}^{s *}(\mathbf{k})=h_{i j}^{s}(-\mathbf{k})$. Next, we can use a circular polarization basis to define the left and right polarizations as

$$
p_{i j}^{R} \equiv \frac{1}{\sqrt{2}}\left(p_{i j}^{+}+i p_{i j}^{\times}\right) \quad \text { and } \quad p_{i j}^{L} \equiv \frac{1}{\sqrt{2}}\left(p_{i j}^{+}-i p_{i j}^{\times}\right)=\left(p_{i j}^{R}\right)^{*}
$$


where $p_{i j}^{+}, p_{i j}^{\times}$are the two linear polarization tensors and $p_{i j}^{R}, p_{i j}^{L}$ are polarizations that rotate in the right and left handed directions, respectively. The TT-conditions also hold for (2.2.4).

The vectors $\hat{\mathbf{k}}=\mathbf{k} /|\mathbf{k}|, \mathbf{e}_{i}^{(1)}$ and $\mathbf{e}_{j}^{(2)}$ are not unique. Any two vectors that satisfy the orthogonality conditions can be used to define the polarization tensors. These can be obtained by rotating through an angle $\theta$ about the unit vector $\hat{\mathbf{k}}$, i.e. $p_{i j}^{s \prime}=$ $e^{ \pm 2 i \theta} p_{i j}^{s}(\mathbf{k})$, with $(s=L, R)$. This corresponds to a symmetry in terms of a rotation by $180^{\circ}$, in terms of which we define the helicity of the gravitational waves to be $h= \pm 2$ for the right and left circular polarizations, respectively. From this we can see that the axes of linear polarization are inclined by $90^{\circ} / h=45^{\circ}$. In standard Einstein gravity the amplitude of the left and right states are the same. But, as we will see later, this is not true when we allow parity violating corrections to Einstein's gravity.

\subsection{Primordial gravitational waves}

In this section we depart from the linearised theory by treating gravitational waves as perturbations over a slowly varying curved spacetime. Let us consider the quadratic action for canonical Einstein gravity in (1.1.14). Perturbing the metric around an FRW background gives (Appendix A.6)

$$
S_{G R}^{(2)}=\frac{M_{P l}^{2}}{8} \int \mathrm{d} \eta \mathrm{d}^{3} x a^{2}\left[\left(h_{i j}\right)^{\prime}\left(h^{i j}\right)^{\prime}-\partial_{k} h_{i j} \partial^{k} h^{i j}\right]
$$

where $\eta$ denotes the conformal time and we omit summation over the polarizations. We define the Fourier transform for tensor perturbations as

$$
h_{i j}(\mathbf{x}, \eta)=\frac{1}{(2 \pi)^{\frac{3}{2}}} \int \mathrm{d}^{3} k \sum_{s} h^{s}(\mathbf{k}, \eta) p_{i j}^{s}(\mathbf{k}) e^{i \mathbf{k} \cdot \mathbf{x}} .
$$

Substituting for (2.3.2) into (2.3.1), and expressing our result in terms of the canonically normalized mode functions ${ }^{1}$

$$
\mu_{s}=\frac{M_{P l}}{2} a h_{s}
$$

we find

$$
S_{G R}^{(2)}=\frac{1}{2} \sum_{s} \int \mathrm{d} \eta \mathrm{d}^{3} k\left[\left(\mu_{s}^{\prime}\right)^{2}-\left(k^{2}-\frac{a^{\prime \prime}}{a}\right) \mu_{s}^{2}\right] .
$$

This is the action for a harmonic oscillator with a time-dependent frequency $\omega^{2}(k, \eta) \equiv$ $k^{2}-a^{\prime \prime} / a$. Therefore, we can proceed to quantize the modes as we would do in the

\footnotetext{
${ }^{1}$ Here we use the conventions in [37].
} 
case of a scalar field. We choose to work with the tensor perturbations $h_{i j}(\mathbf{x}, \eta)$ which can be decomposed into positive and negative frequency modes (i.e. the fields are expanded as sums of creation and annihilation operators as in [42])

$$
\begin{aligned}
h_{i j}(\mathbf{x}, \eta) & =h_{i j}^{+}(\mathbf{x}, \eta)+h_{i j}^{-}(\mathbf{x}, \eta) \\
& =\frac{1}{(2 \pi)^{\frac{3}{2}}} \int \mathrm{d}^{3} k\left[h^{s}(\mathbf{k}, \eta) p_{i j}^{s}(\mathbf{k}) \hat{a}_{s}(\mathbf{k}) e^{i \mathbf{k} \cdot \mathbf{x}}+h^{s *}(\mathbf{k}, \eta) p_{i j}^{s *}(\mathbf{k}) \hat{a}_{s}^{\dagger}(\mathbf{k}) e^{-i \mathbf{k} \cdot \mathbf{x}}\right] \\
& =\frac{1}{(2 \pi)^{\frac{3}{2}}} \int \mathrm{d}^{3} k\left[h^{s}(\mathbf{k}, \eta) p_{i j}^{s}(\mathbf{k}) \hat{a}_{s}(\mathbf{k})+h^{s *}(-\mathbf{k}, \eta) p_{i j}^{s *}(-\mathbf{k}) \hat{a}_{s}^{\dagger}(-\mathbf{k})\right] e^{i \mathbf{k} \cdot \mathbf{x}},
\end{aligned}
$$

where the sum over $s$ has been omitted. To make this more explicit, we can write the above expression in terms of left and right helicity modes as

$$
\begin{aligned}
h_{i j}(\mathbf{x}, \eta) & =\frac{1}{(2 \pi)^{\frac{3}{2}}} \int \mathrm{d}^{3} k\left\{\left[h_{R}(\mathbf{k}, \eta) p_{i j}(\mathbf{k}) \hat{a}_{R}(\mathbf{k})+h_{L}(\mathbf{k}, \eta) p_{i j}^{*}(\mathbf{k}) \hat{a}_{L}(\mathbf{k})\right] e^{i \mathbf{k} \cdot \mathbf{x}}\right. \\
& \left.+\left[h_{R}^{*}(\mathbf{k}, \eta) p_{i j}^{*}(\mathbf{k}) \hat{a}_{R}^{\dagger}(\mathbf{k})+h_{L}^{*}(\mathbf{k}, \eta) p_{i j}(\mathbf{k}) \hat{a}_{L}^{\dagger}(\mathbf{k})\right] e^{-i \mathbf{k} \cdot \mathbf{x}}\right\},
\end{aligned}
$$

where, if we recall from $(2.2 .4), p_{i j}^{L}=\left(p_{i j}^{R}\right)^{*}$. As we saw earlier, gravitational interactions are described by a massless spin-2 particle known as the graviton. The quantization of the gravitational field proceeds the same as in the case of scalar fields. The raising and lowering operators obey the following commutator

$$
\left[\hat{a}_{s}(\mathbf{k}), \hat{a}_{s^{\prime}}^{\dagger}(-\mathbf{q})\right]=(2 \pi)^{3} \delta_{s s^{\prime}} \delta(\mathbf{k}+\mathbf{q}), \quad\left[\hat{a}_{s}(\mathbf{k}), \hat{a}_{s^{\prime}}(-\mathbf{q})\right]=0
$$

with the normalization condition

$$
h^{*}(k, \eta) \partial_{\eta} h(\mathbf{k}, \eta)-h(\mathbf{k}, \eta) h^{*}(\mathbf{k}, \eta) \partial_{\eta} h(\mathbf{k}, \eta)=-\frac{2 i}{M_{P l} a^{2}(\eta)},
$$

where $\hat{a}_{s}(\mathbf{k})|0\rangle=0$ annihilates the initial free vacuum state which is usually called the Bunch-Davies vacuum state and it is defined as the one that is annihilated by the positive mode

$$
h_{i j}^{+}|0\rangle=0 \quad \text { and } \quad\langle 0| h_{i j}^{-}=0 .
$$

The inverse Fourier transform can be read off directly from (2.3.5), as

$$
\begin{aligned}
h_{i j}(\mathbf{k}, \eta) & =h_{i j}^{+}(\mathbf{k}, \eta)+h_{i j}^{-}(\mathbf{k}, \eta) \\
& =\left[h(\mathbf{k}, \eta) p_{i j}^{s}(\mathbf{k}) \hat{a}_{s}(\mathbf{k})+h^{*}(-\mathbf{k}, \eta) p_{i j}^{s *}(-\mathbf{k}) \hat{a}_{s}^{\dagger}(-\mathbf{k})\right] .
\end{aligned}
$$

The two-point function is then defined to be 


$$
\left\langle h_{i j}(\mathbf{k}, \eta) h^{l m}(-\mathbf{q}, \eta)\right\rangle=P_{i j, l m}(\mathbf{k})(2 \pi)^{3} \delta(\mathbf{k}+\mathbf{q}),
$$

where we have adopted the notation in $[42,43]$

$$
P_{i j, l m}(\mathbf{k})=|h(\mathbf{k}, \eta)|^{2} \Pi_{i j, l m}(\mathbf{k}), \quad \text { with } \quad \Pi_{i j, l m}(\mathbf{k})=p_{i j}^{s}(\mathbf{k}) p_{i j}^{s *}(\mathbf{k}) .
$$

Finally, the dimensionless tensor power spectrum is given by

$$
P_{h}(k)=\frac{k^{3}}{2 \pi^{2}} P_{i j, i j}
$$

To evaluate the two-point function we need to know the form of the solutions. The equations of motion for the action in (2.3.1), are given by

$$
h^{\prime \prime}(\mathbf{k}, \eta)+2 \frac{a^{\prime}}{a} h^{\prime}(\mathbf{k}, \eta)+k^{2} h(\mathbf{k}, \eta)=0
$$

where $\mathbf{k}=k=2 \pi a / \lambda=a f$ are the comoving momenta and $f$ is the frequency of the gravitational wave. In terms of the canonically normalized field $\mu(\mathbf{k}, \eta)$, we have

$$
\mu^{\prime \prime}(\mathbf{k}, \eta)+\left(k^{2}-\frac{a^{\prime \prime}(\eta)}{a(\eta)}\right) \mu(\mathbf{k}, \eta)=0
$$

This way one can rewrite (2.3.14) as the equation of a harmonic oscillator with a negative time-dependent mass term $m^{2}(\eta)=-a^{\prime \prime} / a=-a^{2}\left(2 H^{2}+\dot{H}\right)$. From this we see that $m \propto a H$ where $(a H)^{-1}$ is the comoving horizon (i.e. the size of the observable Universe). As this equation cannot be always solved analytically, this enalbes us to split the problem into two regimes, as follows. At subhorizon scales $k^{2} \gg a^{\prime \prime} / a \Rightarrow k \gg a H$ the wavelength of the modes is well within the horizon $\lambda=a / k \ll H^{-1}$ therefore, we can neglect the friction term in (2.3.14). This gives just the equation for a harmonic oscillator, which admits plain wave solutions known as the Bunch-Davies vacuum solution (here we take the positive frequency mode), where gravitons propagate with a speed $c=1$, given by

$$
\lim _{|k \eta| \rightarrow \infty} \mu^{s}(\mathbf{k}, \eta)=-\frac{e^{-i k \eta}}{\sqrt{2 k}} .
$$

In this limit, spacetime is taken to be approximately flat. We say the wavelength of the fluctuations is too small so they do not "feel" the curvature of spacetime. At superhorizon scales $k^{2} \ll a^{\prime \prime} / a \Rightarrow k \ll a H$ the wavelength of the modes is outside the horizon $\lambda=a / k \gg H^{-1}$ therefore, the negative time-dependent mass in (2.3.15) dominates. At this limit the solution is a linear combination of a growing and a decaying mode 


$$
\mu(\mathbf{k}, \eta)=c_{1} a(\eta)+c_{2} a(\eta) \int \mathrm{d} \eta a^{-2}(\eta)
$$

In de Sitter space $\left(a^{\prime \prime} / a=2 / \eta^{2}\right)$, equation (2.3.15) has the exact solution

$$
\mu(\mathbf{k}, \eta)=\beta_{1} \frac{e^{-i k \eta}}{\sqrt{2 k}}\left(1-\frac{i}{k \eta}\right)+\beta_{2} \frac{e^{i k \eta}}{\sqrt{2 k}}\left(1+\frac{i}{k \eta}\right)
$$

where we can use the initial condition in (2.3.16) to fix the constant parameters to $\beta_{1}=1$ and $\beta_{2}=0$, giving

$$
\mu(\mathbf{k}, \eta)=\frac{e^{-i k \eta}}{\sqrt{2 k}}\left(1-\frac{i}{k \eta}\right)
$$

From this, the superhorizon solution is

$$
\lim _{|k \eta| \rightarrow 0} \mu(\mathbf{k}, \eta)=-\frac{i}{\sqrt{2 k^{3}} \eta}
$$

We can express the solution in terms of the tensor fluctuations $h(\mathbf{k}, \eta)$, by using (2.3.3) and that $a=-(H \eta)^{-1}$ in de Sitter, giving

$$
h(\mathbf{k}, \eta)=-\frac{H \eta}{M_{P l} \sqrt{k}}\left(1-\frac{i}{k \eta}\right) e^{-i k \eta}
$$

We find that outside the horizon the quantum fluctuations are constant, i.e. we say they "freeze" which can be seen by taking the small scale limit of the solution in $(2.3 .21)$

$$
\lim _{|k \eta| \rightarrow 0} h(\mathbf{k}, \eta)=\frac{i H}{M_{P l} k^{\frac{3}{2}}}
$$

From this we see that the amplitude of tensor fluctuations at superhorizon scales is proportional to the Hubble parameter $H$, which can be used to define the energy scale of inflation. We can now obtain the power spectrum which is calculated to be

$$
P_{h}=\frac{k^{3}}{2 \pi^{2}} P_{i j, i j}=\frac{k^{3}}{2 \pi^{2}} \frac{H^{2}}{k^{3}} p_{i j}^{s}(\mathbf{k}) p_{i j}^{s}(\mathbf{k})=\frac{2}{\pi^{2}} \frac{H^{2}}{M_{P l}^{2}},
$$

where we have summed over the two polarizations. From this we see that during inflation, due to the rapid expansion of spacetime, quantum fluctuations of spacetime are stretched to classical scales. The power spectrum is scale-invariant if the Hubble parameter is constant, i.e. all the modes have the same amplitude $\sim H$, or nearly 
scale-invariant if the Hubble parameter is allowed to slowly vary during inflation. At horizon exit the tensor fluctuations freeze and remain unchanged till they re-enter the horizon, at some later time, and start to oscillate. This could be during the radiation or matter domination era.

As we do not have measurements of the tensor power spectrum we usually normalize the tensor amplitudes against measurements of the scalar amplitudes by defining the tensor-to-scalar ratio

$$
r \equiv \frac{P_{h}(k)}{P_{s}(k)}
$$

In terms of slow-roll inflation the scalar-tensor ratio is given by $r=16 \epsilon$. Additionally, in quasi-de Sitter there will be a mild scale dependence due to that the Hubble parameter is allowed to vary. We quantify this in terms of the scalar and tensor spectral indexes, which are defined as [40]

$$
n_{s}-1 \equiv \frac{d \ln P_{s}}{\mathrm{~d} \ln k} \quad \text { and } \quad n_{t}=\equiv \frac{d \ln P_{h}}{\mathrm{~d} \ln k},
$$

respectively. One can show that to first-order in slow-roll parameters these can be expressed as [40]

$$
n_{s}-1=2 \eta-4 \epsilon, \quad n_{t}=-2 \epsilon .
$$

The current bound from the Planck collaboration [33] on the scalar spectral index is $n_{s}=0.956 \pm 0.004$, which is very close to unity. The spectral indexes could be a useful tool to help distinguish between the predictions from different models of inflation, as any deviation from scale invariance will require $n_{s} \neq 1$ and $n_{t} \neq 0$. Additionally, any variation of the spectral index (or running) would have to arise at second-order in the slow-roll parameters and therefore it is expected to be very suppressed.

One can relate the scalar-tensor ratio and the tensor spectral index via the consistency relation

$$
r=-8 n_{t}
$$

which would be useful in the future, if we could measure the scale dependence of tensor modes. Next, we examine the observational consequences for primordial gravitational waves.

\subsection{Detecting primordial gravitational waves}

In the previous section we saw that the theory of inflation predicts the existence of a nearly scale invariant primordial stochastic gravitational wave background $[44,45]$. 
Their amplitude is described by the scalar-tensor ratio $r$, which is defined as the ratio between the scalar and tensor power spectra. The current best upper bound we have is by using a combination of data from BAO, BICEP2 and Keck Array, $r<0.06$ at the pivot scale $k_{*}=0.05 \mathrm{Mpc}^{-1}$ [33]. A definite detection of primordial gravitational waves will help to determine the energy scale of inflation, which we currently know little about, and can be anywhere between the TeV and GUT scale. Additionally it could help us discriminate between inflationary models that produce different observational signatures for gravitational waves.

One way to examine this is by studying the statistical properties of the fluctuations generated during inflation. In particular, tensor perturbations can leave a characteristic imprint in the CMB polarization with a curl-like pattern known as B-modes [46,47], shown in Figure (2.2). In what follows, we give a brief introduction of the CMB and how it can be used to identify the B-mode pattern.

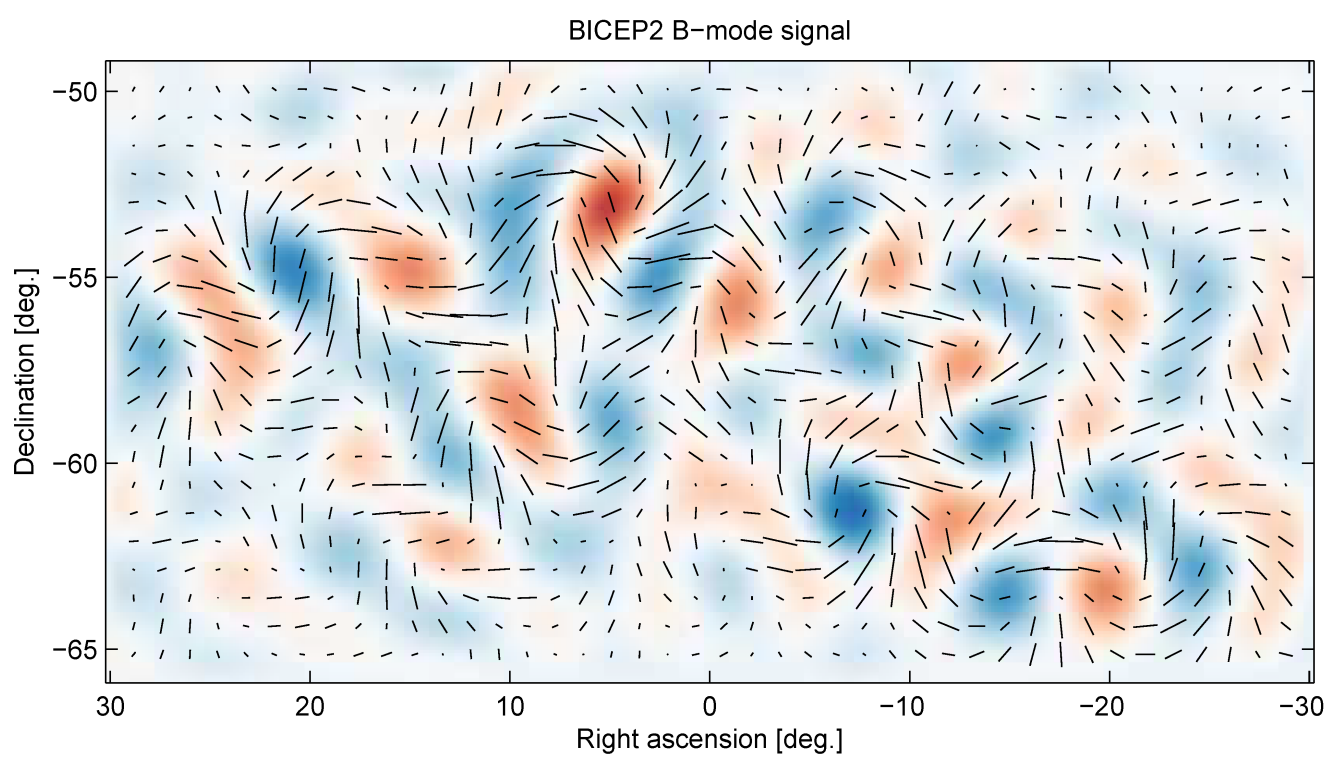

Figure 2.2: The B-mode pattern, thought to be generated by gravitational waves in the CMB, which was observed with the BICEP2 telescope. The twisting behaviour of the B-mode pattern in clockwise or anti-clockwise directions is shown in red and blue colour. Image credit: BICEP2 2014 Release Image Gallery

Soon after the Big Bang there was a cosmic soup of highly energetic particles undergoing continuous collisions which resulted in them being in thermal equilibrium, i.e. the average temperature and kinetic energy were the same everywhere. When the Universe had expanded and cooled enough for stable hydrogen to form, around a redshift of $z \sim 1091$ [48], photons were able to decouple from the cosmic plasma and freely travel in the Universe. These are the photons that form the blackbody spectrum that we measure in the CMB with experiments, such as the COBE satellite [49], WMAP [48] and more recently Planck [15]. The data from these experiments has shown that the observable Universe is homogeneous and isotropic with a uniform temperature $T=2.7 \mathrm{~K}$. 

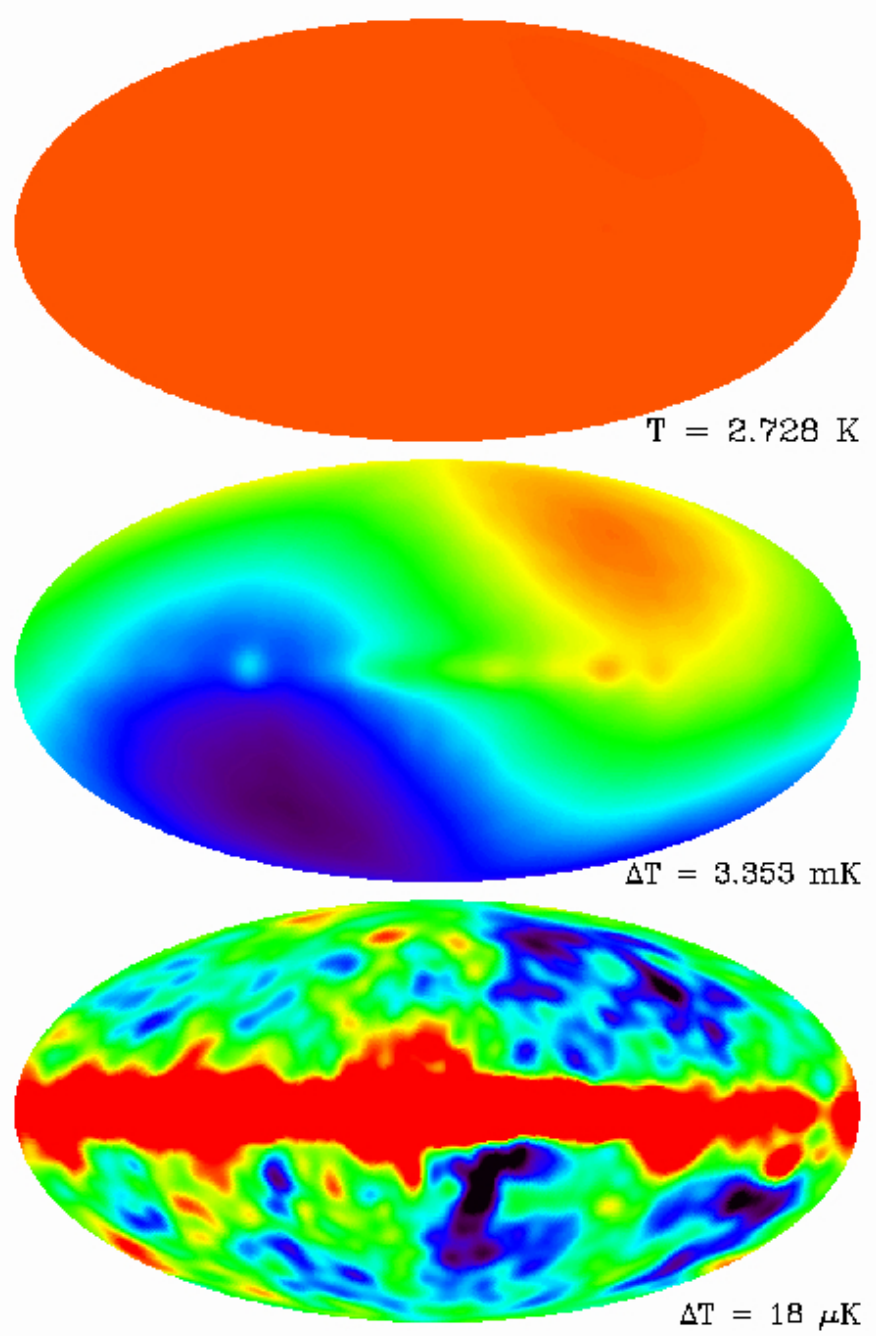

Figure 2.3: The first map shows the uniform temperature of the CMB, as measured by COBE, while the second shows the dipole anisotropy $\Delta T / T \sim 10^{-3}$ at $l=1$ due to the motion of the Earth relative to the frame of the CMB. The third map includes emissions from the Milky way but the dipole contribution has been subtracted. It shows the CMB anisotropies for $l>1$ at $\Delta T / T \sim 10^{-5}$. Image credit: COBE Image Gallery. 
The CMB also contains small temperature anisotropies, see Figure (2.3), which are represented in terms of an expansion in spherical harmonics, given by

$$
\delta T(\hat{n})=T(\hat{n})-\bar{T}(\hat{n})=\sum_{l m} a_{l m} Y_{l m}(\hat{n})
$$

where the multipole moments $l$ are associated with the scale of the fluctuations, $m=-l, \cdots,+l, \bar{T}$ is the background temperature and $\hat{n}$ points the direction in the sky. We define the spherical harmonics as

$$
Y_{l m}(\theta, \phi)=\sqrt{\frac{(2 l+1)}{4 \pi} \frac{(l-m) !}{(l+m) !}} P_{l m}(\cos \theta) e^{i m \phi}
$$

where $P_{l m}$ are associated Legendre polynomials. We quantify the amount of correlation there is at a given angular separation in the sky in terms of the angular power spectrum which, given $l$, is estimated by averaging over all $m$-modes for a single realization of $\delta T$, given by

$$
\mathcal{C}_{l}=\frac{1}{2 l+1} \sum_{m=-l}^{l}\left\langle a_{l m}^{*} a_{l m}\right\rangle
$$

and where the coefficients $a_{l m}$ are determined by the CMB data. This way we can define the CMB angular power spectra for temperature, E-modes and B-modes, respectively as

$$
\mathcal{C}_{l}^{T}=\left\langle a_{l m}^{T *} a_{l m}^{T}\right\rangle, \quad \mathcal{C}_{l}^{E}=\left\langle a_{l m}^{E *} a_{l m}^{E}\right\rangle, \quad \mathcal{C}_{l}^{B}=\left\langle a_{l m}^{B *} a_{l m}^{B}\right\rangle
$$

The TT power spectra have been measured to great precision by the Planck satellite [3], which can be seen in Figure (2.4). On the left we can see that there is greater uncertainty for multipole moments $l \leq 30$ due to cosmic variance. This is because there is an intrinsic uncertainty in the CMB measurements which relates to that we can only measure a set amount of fluctuations per given scale, determined by $2 l+1$ in (2.4.3). From this it is easy to see what we can expect less variance at small scales (large $l$ ) and larger variance at large scales (small $l$ ), i.e. there is only a set amount of modes over which to average at large scales, resulting to greater uncertainty.

Most of the photons that we measure from the CMB have no net polarization i.e. the temperature component of the $\mathrm{CMB}$ radiation is completely unpolarized. DESI [50] and subsequent CMB experiments found that the CMB radiation is partially $(10 \%)$ polarized due to Thomson scattering. The predominant mechanism that sources a quadrupole anisotropy in the CMB is due to the scalar (density) oscillations known as E-modes [46,47]. At the end of the recombination era, light from the hot and cold spots of the quadrupole temperature anisotropy in the CMB is scattered by electrons with different intensities resulting in a net linear polarization. Quadrupole anisotropy can also be sourced by vorticity modes (vectors) but 


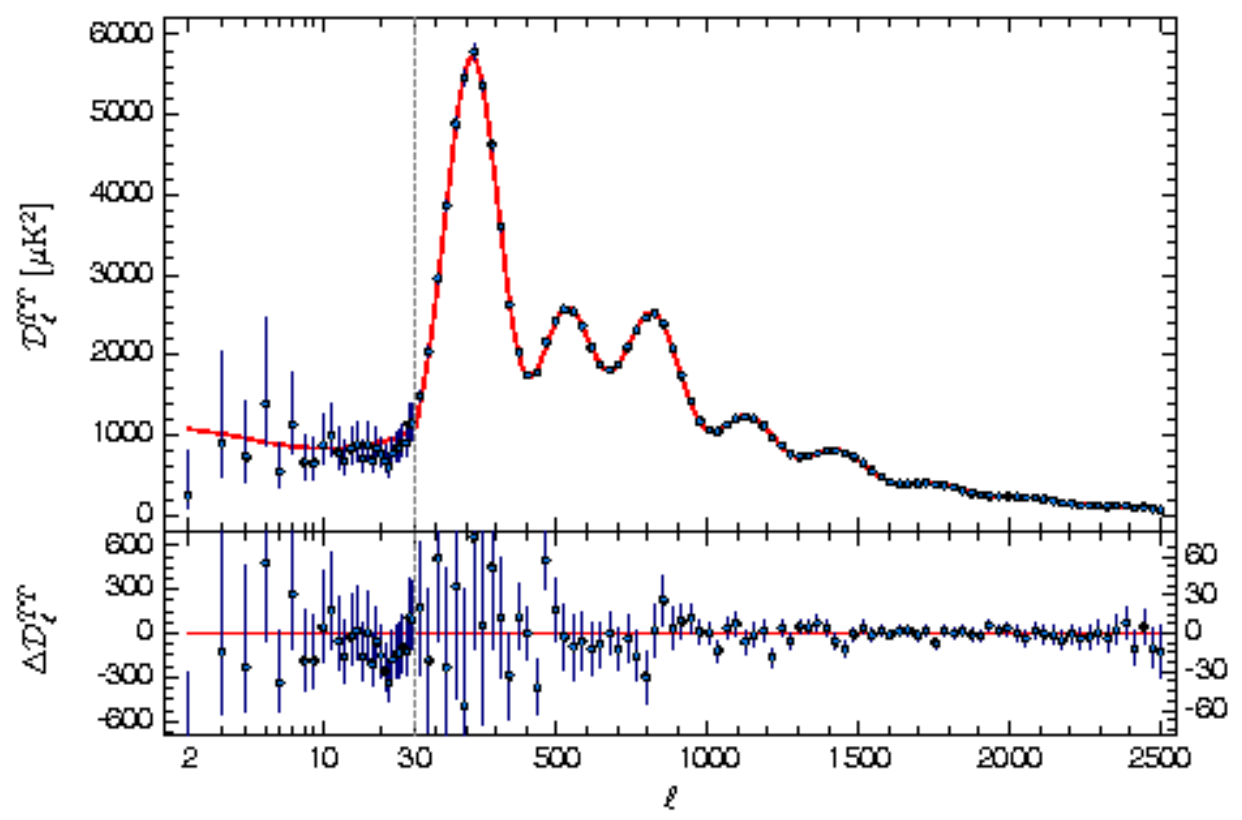

Figure 2.4: The Planck (2015) temperature power spectrum [3]. The vertical axis displays the quantity $\mathcal{D}_{l}=l(l+1) \mathcal{C}_{l} / 2 \pi$. The multipole moments $l$ are displayed on the horizontal axis. The scale is treated logarithmically till $l=50$ and linearly after that. Large angles correspond to small $l$. The error bars show uncertainties of $\pm 1 \sigma$ due to cosmic variance. We use the peaks to extact information about the cosmological parameters. The damping tail at high multipoles is because decoupling of photons from baryons does not happen instantaneously. 
it is assumed to be negligible. More interestingly, a quadrupole anisotropy can be sourced by gravitational waves as their effect is to distort spacetime. Depending on the direction in which a gravitational wave squeezes and stretches spacetime, it can generate both $E$-modes and $B$-modes. The latter cannot be sourced by scalar fluctuations therefore, it is said that detection of B-modes will be a definite confirmation of the production of gravitational waves from inflation [51].

The CMB polarization pattern is usually described by Stoke parameters in analogy to the way we express polarization of fields in electromagnetism. The $E$-modes are curl free $\nabla \times E=0$, similar to the gradient of a potential, while the $B$-modes are divergence free $\nabla \cdot B=0$. We do not consider this analysis in detail here. Instead we can simplify these arguments by visualizing a plane wave propagating at the direction of a vector $\mathbf{k}$. The $E$-mode polarization is parallel or perpendicular to the direction of $\mathbf{k}$, while the $B$-modes are at $\pm 45^{\circ}$ with respect to the direction $\mathbf{k}$, as shown in Figure (2.5).
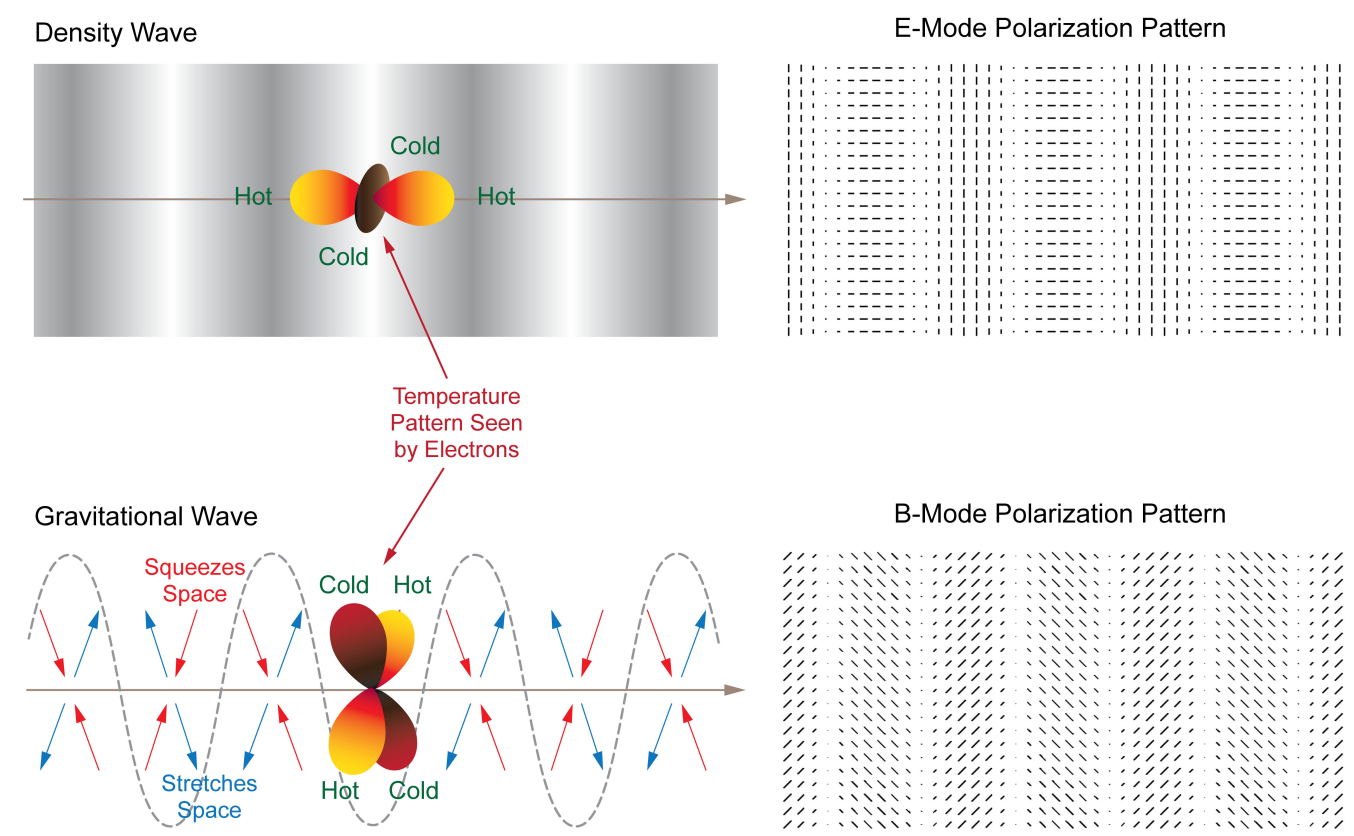

Figure 2.5: The mechanism that produces E- and B-modes in the CMB. Image credit: BICEP2 2014 Release Image Gallery

We can gain more information by cross-correlating the different CMB radiation modes. The angular cross-power spectra are

$$
\mathcal{C}_{l}^{T E}=\left\langle a_{l m}^{T *} a_{l m}^{E}\right\rangle, \quad \mathcal{C}_{l}^{T B}=\left\langle a_{l m}^{T *} a_{l m}^{B}\right\rangle, \quad \mathcal{C}_{l}^{E B}=\left\langle a_{l m}^{E *} a_{l m}^{B}\right\rangle
$$

Under parity inversion the $T$ and the $E$ modes stay the same, while the $B$-modes have the opposite parity as they transform as $B \rightarrow-B$. Therefore the last two cross-correlations vanish $\mathcal{C}_{l}^{T B}=\mathcal{C}_{l}^{E B}=0$, if the physical processes that give rise to the $\mathrm{CMB}$ polarizations are parity conserving and only the first four power spectra in 
(2.4.4) and (2.4.5) contribute to the CMB statistics [34]. On the other hand, if parity violating processes are present, we expect to find an additional two cross-correlating power spectra, which are the last two in (2.4.5).

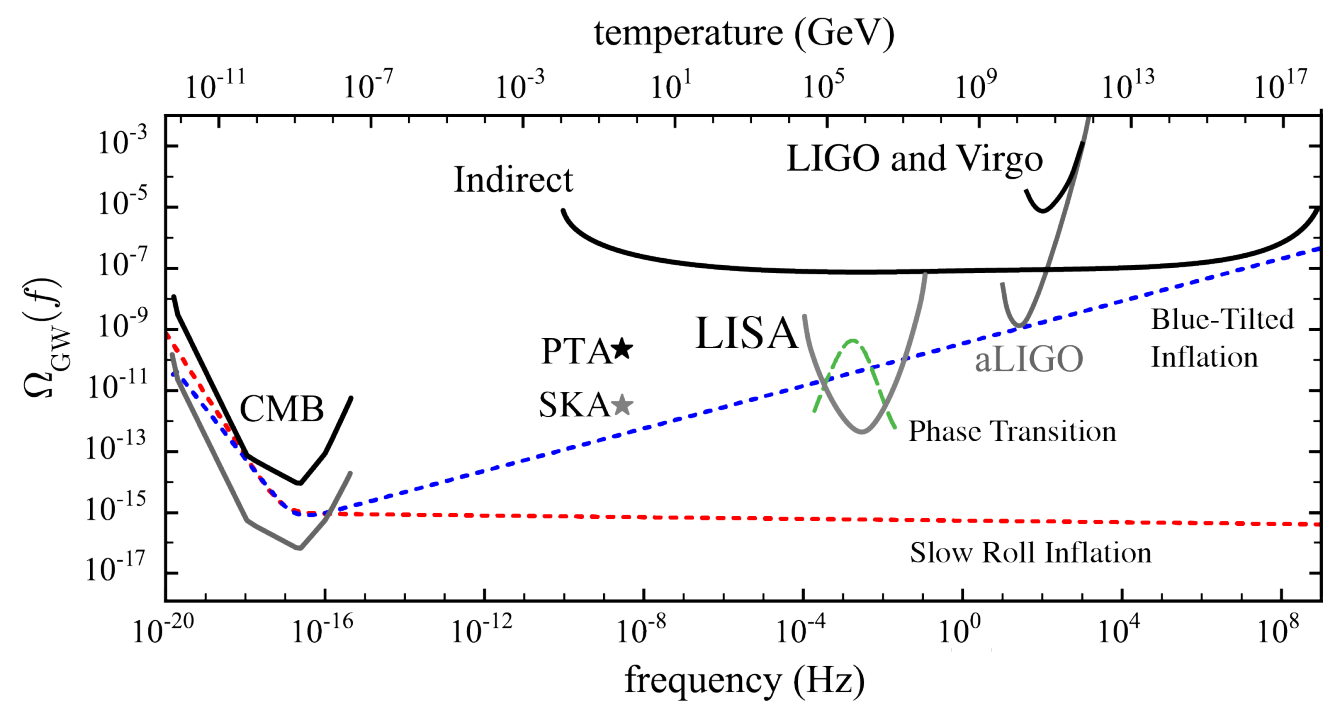

Figure 2.6: Here we see the sensitivity of LISA to a stochastic GW background relative to other experiments [4]. The observational predictions from inflation, maximum blue-tilted spectrum and electroweak phase transition are indicated with red, blue and green dashed lines. Current and future sensitivity of experiments is indicated by black and grey curves. The sensitivities displayed are from LIGO and Virgo, aLIGO, PTA and SKA. On the left of the diagram we can see the sensitivity for current and future CMB experiments where the black curve shows the current bound for $r<0.1$, while the grey curve shows the projected sensitivity for future $C M B$ experiments $r<0.001$. Finally, the sensitivity to measurements from Big Bang Nucleosynthesis is displayed by the indirect curve.

So far we have not detected $B B$ power spectra and consequently $E B$ and $T B$ power spectra. $\mathrm{CMB}$ measurements are inhibited by galactic foregrounds such as galactic synchrotron radiation, dust from thermal emission in the interstellar medium, detector noise and systematic errors. There can also be lensing-induced Bmodes which is due to photons traversing the gravitational structure of the Universe at large scales $[52,53]$.

In 2014 the BICEP2 Collaboration which operated from the South Pole presented what was thought to be evidence for B-mode polarization in the CMB at the level of $r \simeq 0.2[54,55]$, which was then followed by a joint analysis with Planck in 2015 [56] concluding that the entire signal could be attributed to cosmic dust.

So far we can extract the signal from galactic foregrounds by using multi-frequency techniques. We hope that improvements on detector noise and de-lensing will help in the future to probe B-Modes with experiments that are sensitive to a scalar-tensor ratio of $r \sim 10^{-3}$.

There are several current and forthcoming experiments which are especially equipped to look for B-modes such as the Polarbear experiment in Chile [57], the 
AdvAct telescope at the Atacama desert of northern Chile [58], CLASS at the Atacama desert of Chile [59] and the balloon-born experiment SPIDER [60]. Several next generation experiments have been proposed, such as the LiteBIRD satellite [61], PRISM [62] and many more.

It may also be possible, in the future to detect a primordial stochastic background with interferometers. Figure (2.6) shows the sensitivity curves of current and future experiments [4]. The primordial gravitational wave spectrum predicted by standard inflationary modes is way below the sensitivity of the space interferometer LISA. On the other hand, models that predict a maximally blue-tilted spectrum could enter the sensitivity of detectors (see references therein). In this work we consider models with rich phenomenology that can produce observational signatures which may be enter the sensitivity curves of future CMB experiments, as well they may be detectable with interferometers.

\subsection{Non-gaussianity}

In Sections (1.3) and (2.3) we saw that inflation predicts a nearly scale invariant spectrum of scalar and tensor fluctuations. We also found in Section (2.4) that the temperature distribution of the $\mathrm{CMB}$ is very close to Gaussian i.e. on average there is the same amount of colder and hotter patches in the CMB sky. If the primordial density perturbations were sourced by quantum fluctuations then we do expect them, at lowest order, to obey a Gaussian distribution. On the other hand, non-Gaussianities, if present in the $\mathrm{CMB}$, will be the result of interactions and non-linear dynamics of the quantum fields, which we represent as $n$-point functions.

If the spectrum is Gaussian then all the information about the perturbations is contained in the two-point function and we expect that the three-point function or higher odd $n$-point correlators to vanish. Also, using Wick's theorem one can show that all the even $n$-point functions can be represented as products of the two-point function.

So far, CMB experiments have not detected any non-Gaussianity, but if we do this could be used to probe the physics of the early Universe, as different classes of inflationary models predict very specific shapes of non-Gaussianity in the temperature and polarization anisotropies of the CMB [63].

It is worth mentioning that this should not be confused with large scale nonGaussianity, i.e. that there is much more empty space in the late-time Universe compare to regions where there is high density of concentration of matter, such as galaxies and galaxy clusters. Our present universe is highly non-Gaussian due to the non-linear effects of gravity which have been accumulated over time. This is know as secondary non-Gaussianity and it does not concern us in this work.

The methods for studying non-Gaussianities were developed in [42,64]. Here we introduce the key steps and notation needed for the subsequent sections. We are interested to compute the leading-order contribution to the three-point function in the interaction picture of the field theory. In curved spacetime, the quantized fields are taken, in general, to propagate on some classical time-dependent background. When working at quadratic order the usual prescription is to split the field, say 
$u(x, \tau)$, into a classical part $u_{b}$ (the background field) and a quantum part $u_{0}$ (perturbation). Following a similar procedure, as we are interested into the three-point function, it is convenient to split the Hamiltonian into three parts,

$$
\mathcal{H}=\mathcal{H}_{b}+\mathcal{H}_{0}+\mathcal{H}_{\text {int }}
$$

where the background Hamiltonian $\mathcal{H}_{b}$ contains contributions to the background (i.e. such as the metric $g_{\mu \nu}$, the scale factor $a(t)$, the Hubble parameter and background quantities associated with the inflaton field) which are c-numbers and commute with everything. We expand the Hamiltonian around the classical background and split the perturbation Hamiltonian into the free Hamiltonian $\mathcal{H}_{0}$ which contains the contributions to the quadratic part and the interaction Hamiltonian $\mathcal{H}_{\text {int }}$ which contains the $n$-order contributions. The later contributes to the three-point function and is given by

$$
\mathcal{H}_{\text {int }}=-\mathcal{L}_{\text {int }}
$$

where the Lagrangian density $\mathcal{L}_{\text {int }}$ is taken to be $n \geq 3$ order in perturbations of the fields. The time evolution of the state is given by

$$
|0(t)\rangle=T e^{-i \int_{t_{0}}^{t} \mathrm{~d} t^{\prime} \mathcal{H}_{i n t}\left(t^{\prime}\right)}\left|0\left(t_{0}\right)\right\rangle
$$

where the initial time is taken to be in the infinite past $t_{0} \rightarrow-\infty$ and therefore the initial state is defined as $|0\rangle=\left|0\left(t_{0}\right)\right\rangle=|0(-\infty)\rangle$. Since we are interested in super-Hubble fluctuations (i.e. modes that their wavelengths have been stretched to be much larger than the horizon size) the final time $t$ is taken to be when all the modes of interest have left the horizon. Once the integrals have been evaluated we can take the limit $t \rightarrow 0$ to find the contribution to the three-point function at the end of inflation.

In practise, the three-point function is evaluated in the vacuum state of the free theory at $t_{0} \rightarrow-\infty$, which is identified with the vacuum state of the full theory. Any non-trivial vacuum fluctuations which may be generated by excitations from interaction terms are smoothed out by using the $i \epsilon$ prescription, which also regulates oscillatory behaviour of the integrand at the infinite past, making the integral well defined (for more details see the pedagogical treatment in [65]).

We denote the three-point function as a product of field operators $W_{\text {int }}(t)$, i.e. in terms of tensor perturbations we have that $\left\langle W_{\text {int }}(t)\right\rangle$ is equivalent to $\left\langle h^{3}(\tau)\right\rangle=$ $\left\langle h_{t m}\left(\mathbf{k}_{1}, \tau\right) h_{n r}\left(\mathbf{k}_{2}, \tau\right) h_{p v}\left(\mathbf{k}_{3}, \tau\right)\right\rangle$. Then, the three-point function is given by in terms of time and anti-time ordered integrals in (2.5.3), with the fields sandwiched between the Bunch-Davies vacua. This reads 


$$
\begin{aligned}
\left\langle W_{\text {int }}(t)\right\rangle & =\left\langle\left[\bar{T} \exp \left(i \int_{-\infty(1-i \epsilon)}^{t} \mathrm{~d} t^{\prime} \mathcal{H}_{\text {int }}\left(t^{\prime}\right)\right) W_{\text {int }}(t)\right.\right. \\
& \left.\left.\times T \exp \left(-i \int_{-\infty(1-i \epsilon)}^{t} \mathrm{~d} t^{\prime} \mathcal{H}_{\text {int }}\left(t^{\prime}\right)\right)\right]\right\rangle .
\end{aligned}
$$

Since the interaction Hamiltonian is small, the time-ordered arguments (exponentials) can be Taylor expanded (using a Dyson series), i.e at next to leading order in the expansion we have

$$
\bar{T} \exp \left(i \int_{-\infty(1-i \epsilon)}^{t} \mathrm{~d} t^{\prime} \mathcal{H}_{i n t}\left(t^{\prime}\right)\right)=1-i \int_{-\infty(1-i \epsilon)}^{t} \mathrm{~d} t^{\prime} \mathcal{H}_{\text {int }}\left(t^{\prime}\right)+\cdots
$$

where each order in $H_{\text {int }}$ is a vertex and carries a time integral $t$ and a space integral, from the definition of $H_{\text {int }}$, which in Fourier space it enforces momentum conservation. We can now substitute (2.5.5) into (2.5.4) and after some manipulation one finds the next-to leading order term is (a single vertex)

$$
\left\langle W_{\text {int }}(t)\right\rangle=\operatorname{Re}\left\langle\left[-2 i W_{\text {int }}(t) \int_{-\infty(1-i \epsilon)}^{t} \mathrm{~d} t^{\prime} \mathcal{H}_{\text {int }}\left(t^{\prime}\right)\right]\right\rangle,
$$

where we have used Hermiticity. Once the interaction Hamiltonian is known, we can substitute it into the expression above. Without delving into the details, we would like to point out that the interaction Hamiltonian is constructed by the interaction picture fields (and background quantities). These are defined with Bunch-Davies initial conditions so that at the infinite past $t \rightarrow-\infty$ they can be identified with the fields of the quadratic free theory. All that this means is that the interaction fields are solutions to the linearized equations of motion.

To evaluate the right-hand side of (2.5.6) one needs to expand the fields as sums of creation and annihilation operators and contract them. One can then substitute for the solutions from the equations of motion. We are usually interested at the leading-order contribution to the three-point function of the fluctuations, which in the case of tensors, this is given by

$$
\begin{aligned}
\left\langle h^{3}\left(\tau_{0}\right)\right\rangle & =\left\langle h_{t m}\left(\mathbf{k}_{1}, \tau_{0}\right) h_{n r}\left(\mathbf{k}_{2}, \tau_{0}\right) h_{p v}\left(\mathbf{k}_{3}, \tau_{0}\right)\right\rangle \\
& \left.=-i \int_{-\infty}^{\tau_{0}} \mathrm{~d} \tau\left\langle h_{t m}\left(\mathbf{k}_{1}, \tau_{0}\right) h_{n r}\left(\mathbf{k}_{2}, \tau_{0}\right) h_{p v}\left(\mathbf{k}_{3}, \tau_{0}\right), \mathcal{H}_{i n t}(\tau)\right]\right\rangle
\end{aligned}
$$

where $\tau$ is the conformal time. The usual prescription is to evaluate the correlation functions by employing the "in-in" formalism [42], which is suitable for calculating correlation functions in time-dependent backgrounds (as opposed to calculating the "in-out" matrix element). We do not review the formal derivation here. For more details see [65]. 
We calculate contractions between two fields by normal ordering. This ensures that all annihilation operators stand to the left of all creation operators, so that they hit the vacuum as in (2.3.9). These terms drop out leaving just two contracted fields. The details are shown in the Appendix (A.14).

Next we look at how to make contact with observations. The terms in the interaction Hamiltonian are mode-dependent and contribute to the bispectrum shape. It is convenient to define the bispectrum via the three-point correlation function in Fourier space. For curvature perturbations, this is defined as

$$
\left\langle\mathcal{R}\left(\mathbf{k}_{1}\right) \mathcal{R}\left(\mathbf{k}_{2}\right) \mathcal{R}\left(\mathbf{k}_{3}\right)\right\rangle=(2 \pi)^{3} \delta^{(3)}\left(\mathbf{k}_{1}+\mathbf{k}_{2}+\mathbf{k}_{3}\right) B_{\mathcal{R}}\left(\mathbf{k}_{1}, \mathbf{k}_{2}, \mathbf{k}_{3}\right)
$$

where the delta function ensures momentum is conserved $\left(\mathbf{k}_{1}+\mathbf{k}_{2}+\mathbf{k}_{3}=0\right)$ so that the amplitudes of the three momenta form the sides of a closed triangle in Fourier space. The function $B_{\mathcal{R}}$ depends on the momenta and, due to rotational invariance, it has only two independent variables, namely the ratios of $k_{2} / k_{1}$ and $k_{3} / k_{2}$. In general, the bispectrum takes maximal values for the three modes that have the strongest interaction. This is best described by assigning a shape to the bispectrum, as shown in Figure (2.7)

(a) squeezed triangle $\left(k_{1} \sim k_{2}>>k_{3}\right)$

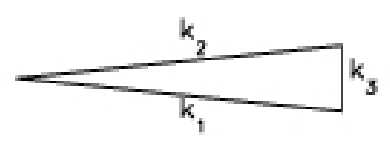

(d) isosceles triangle $\left(k_{1}>k_{2}=k_{3}\right)$

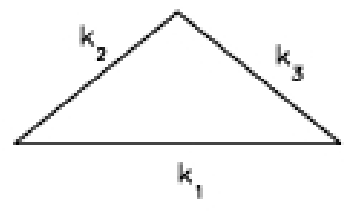

(b) elongated triongle $\left(k_{1}=k_{2}+k_{3}\right)$

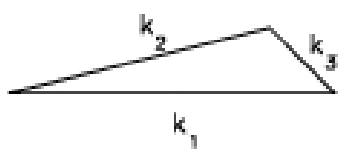

(e) equilateral triangle

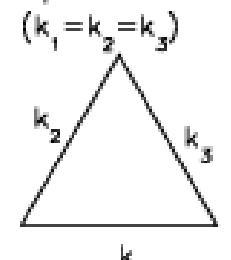

(c) folded triangle $\left(k_{1}=2 k_{2}=2 k_{3}\right)$
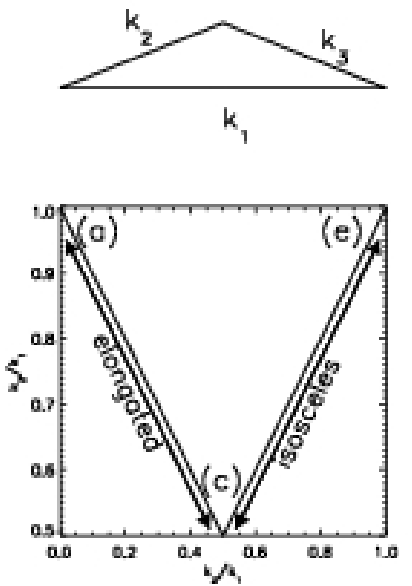

Figure 2.7: Shapes of the bispectrum [5] with momenta satisfying $\mathbf{k}_{3} \leq \mathbf{k}_{2} \leq \mathbf{k}_{1}$.

To describe the shapes, it is useful to define the local non-Gaussianity for a primordial curvature perturbation $\mathcal{R}(\mathbf{x})$, as in [66], via the relation

$$
\mathcal{R}(\mathbf{x})=\mathcal{R}_{L}(\mathbf{x})+\frac{3}{5} f_{\mathrm{NL}}^{\text {local }}\left[\mathcal{R}_{L}^{2}(\mathbf{x})-\left\langle\mathcal{R}_{L}^{2}(\mathbf{x})\right\rangle\right]
$$


In this way $\mathcal{R}(\mathbf{x})$ is defined as a function of the position, locally in real space, as opposed to momentum space. The factor $3 / 5$ is for agreement with the literature as this model was first considered in terms of the Newtonian potential $\Phi=3 / 5 \mathcal{R}$. This relation describes perturbations beyond linear order in $\mathcal{R}_{L}(\mathbf{x})$ which is the linear Gaussian perturbation. The variance is subtracted from the quadratic term, in the brackets, to ensure that the expectation value of $\langle\mathcal{R}(\mathbf{k})\rangle$ vanishes [67]. The parameter $f_{N L}$ describes the amount of non-linearity of the three-point function $\left\langle\mathcal{R}^{3}(\mathbf{x})\right\rangle$. The parameter $f_{N L}$ is a constant for slow-roll inflation or it can be scale dependent, i.e. $f_{N L}=f_{N L}\left(k_{1}, k_{2}, k_{3}\right)$, if the slow-roll condition is not obeyed [68]. In momentum space this can be expressed as

$$
\mathcal{R}(\mathbf{k})=\mathcal{R}_{L}(\mathbf{k})+\frac{3}{5} f_{\mathrm{NL}} \frac{1}{(2 \pi)^{3}} \int \mathrm{d}^{3} \mathbf{q} \mathcal{R}_{L}(\mathbf{q}) \mathcal{R}_{L}(\mathbf{k}-\mathbf{q})
$$

Using (2.5.8) and (2.5.9) one can obtain the expression for the local bispectrum

$$
B_{\mathcal{R}}^{\text {local }}\left(\mathbf{k}_{1}, \mathbf{k}_{2}, \mathbf{k}_{3}\right)=\frac{6}{5} f_{\mathrm{NL}}^{\text {local }}\left(P_{\mathcal{R}}\left(k_{1}\right) P_{\mathcal{R}}\left(k_{3}\right)+2 \text { perms }\right) .
$$

In the case where the spectrum is scale-invariant, i.e. $\mathcal{P}_{\mathcal{R}}(k)=A / k^{3}$ where $A=$ const., then the bispectrum is found to be [37]

$$
B_{\mathcal{R}}^{\text {local }}\left(\mathbf{k}_{1}, \mathbf{k}_{2}, \mathbf{k}_{3}\right)=\frac{6}{5} f_{\mathrm{NL}}^{\text {local }} A^{2}\left(\frac{1}{\left(k_{1} k_{3}\right)^{3}}+2 \text { perms }\right) .
$$

In general, the bispectrum takes maximal values when the three modes have the strongest interaction which leads to different triangle configurations, which can help differentiate between inflationary models. We briefly mention a few of the possible shapes.

In the squeezed limit, also known as the local shape, one of the momenta is taken to be much smaller than the other two, which are taken to be approximately equal $k_{3} \ll k_{1} \sim k_{2}$. During inflation, small wavelengths (large momenta) exit the horizon first while large wavelengths (small momenta) are already super Hubble therefore, classical and conserved. These just contribute to the background dynamics, i.e. their effect is to shift the background by a constant value. From this one finds that in the squeezed limit this reduces into a two-point function between the momenta. As an example, consider the squeezed limit $k_{3} \ll k_{1} \sim k_{2}$ in (2.5.12). The bispectrum for local non-Gaussianity becomes

$$
\lim _{k_{3} \ll k_{1} \sim k_{2}} B_{\mathcal{R}}^{\text {local }}\left(\mathbf{k}_{1}, \mathbf{k}_{2}, \mathbf{k}_{3}\right)=\frac{12}{5} f_{\mathrm{NL}}^{\text {local }} \frac{A^{2}}{\left(k_{1} k_{3}\right)^{3}}=\frac{12}{5} f_{\mathrm{NL}}^{\text {local }} P_{\mathcal{R}}\left(k_{1}\right) P_{\mathcal{R}}\left(k_{2}\right) .
$$

In general multi-field inflation models are known to peak in this shape while nonGaussianity in the squeezed limit for single field models is expected to vanish. This is because of the Maldacena consistency relation for single-field inflation $[64,69]$ 
where they showed that the three-point function can be expressed as the product of two-point functions, given by

$$
f_{\mathrm{NL}}^{l o c a l} \sim \lim _{k_{1} \rightarrow 0}\left\langle\zeta_{k_{1}} \zeta_{k_{2}} \zeta_{k_{3}}\right\rangle \sim\left(n_{s}-1\right)\left\langle\zeta_{k_{2}} \zeta_{-k_{2}}\right\rangle\left\langle\zeta_{k_{3}} \zeta_{-k_{3}}\right\rangle
$$

Therefore, we expect that we should not find non-Gaussianity in the squeezed limit for most single-field inflationary models (i.e. $n_{s} \sim 1$ ). Later on we present a set-up for single-field inflation in which it is possible to enhance the tensor bispectrum in the squeezed limit. The Planck collaboration constraints, using a combination of temperature and polarization data, give $f_{N L}^{\text {local }}=0.8 \pm 5.0$ at $(68 \% \mathrm{CL})$ which corresponds to a very small amount of non-Gaussianity in the CMB at the squeezed limit.

In the case of equilateral non-Gaussianity, one gets a maximal value for the bispectrum when all three modes have equal wavelengths. This is particularly interesting for models with higher-derivative corrections, such as DBI inflation [70], G-inflation [36] and Galileon inflation [71], which are known to be suppressed if one of the modes is outside the horizon. When all the modes are inside the horizon this leads to large non-Gaussianity. These models are known to have non-canonical kinetic terms where fluctuations propagate with non-trivial speeds $c_{s}$. In the nonrelativistic limit one can have $f_{N L} \sim c_{s}^{-2}$ which is constrained by observations to have the lower bound $c s^{2}>0.1[72]$. We say that the bispectrum shape peaks in the equilateral configuration $k_{1}=k_{2}=k_{3}$ (equilateral triangle). The Planck constraints for these shapes are $f_{N L}^{\text {equil }}=-4 \pm 43$ and $f_{N L}^{\text {ortho }}=-26 \pm 21$ at (68\% CL) [73].

There are several excellent reviews on the topic of primordial non-Gaussianity, some of which are $[63,65,67,74-76]$. 


\section{Beyond Einstein gravity}

Effective field theories are at the heart of modern theoretical physics. Physical processes in nature occur at a wide range of energy scales, of which we can experimentally access only the low-energy part. With regard to cosmological applications, in the early universe, we expect the energy scale of the Cosmic Microwave Background radiation to have been several orders of magnitude smaller than that of the Planck scale, where quantum processes are thought to become important [15]. Yet, by continuously improving the sensitivity of experiments, in the future, we may be able to access physical processes at much larger energies and potentially find new physics. While physical processes at very high energies are largely unknown to us, the effective field theory machinery enables theoretical physicists to probe such regimes and make predictions that can be both qualitative but also quantitative. Below, we review this approach.

\subsection{Bottom-up approach}

Quantum gravity is known to be non-renormalizable in four dimensions, meaning the theory loses its predictivity as there can be an infinite amount of corrections and therefore an infinite amount of parameters present in the theory. String theory is considered among the best candidates for a complete theory of quantum gravity. In the so called top-down approach, one can start from a UV complete theory (i.e String theory or SUGRA) and produce a low energy theory in which highenergy physics appear as corrections to the inflaton potential. Here we refrain from discussing such approaches, although they can be phenomenologically interesting, and we focus instead on the so called bottom-up approach, where one assumes no previous knowledge of what the fundamental theory may be.

Since the 1980's, gravity is seen as a low-energy effective field theory $[77,78]$. At low energies classical gravity is a diffeomorphism invariant theory with a spin two particle, the graviton [79]. Quantum effects of gravity are expected to become important when spacetime is strongly curved, making them negligible with regards to current experimental applications. On the other hand, if physical processes in the early Universe took part at very high energy scales, we may be able, in the future, to find evidence of new physics in our data [80]. In particular, the upper bound to the energy scale of inflation, from the Planck collaboration [15], is currently at $H \sim 10^{13} \mathrm{GeV}$. So it is not completely unreasonable to wonder whether inflation could be sensitive to high-energy physics, which can occur at energies $M_{P l} \sim 10^{18}$ 
$\mathrm{GeV}$, or below.

Inflation is seen as a period of rapid expansion of spacetime with $a \sim e^{H t}$. During this process, the wavelengths of highly energetic modes are stretched into lower energy scales. These are the modes that we measure at the end of inflation. The background geometry of spacetime is treated classically and only the perturbations are quantized, which are expected to be strongly coupled when their wavelength is of order $M_{P l}$, i.e. deep within the horizon.

To construct an effective description for inflation we borrow from methods that have been used and tested, for a long time now, in particle physics [81]. At very high energies we expect that heavy degrees of freedom may be excited. These processes are not energetically accessible to our experiments and are said to be offshell, as we can deal only with initial and final states of light particles. In cases where the fundamental theory is known, the usual prescription is to integrate the heavy degrees of freedom out of the Lagrangian. This in turn introduces higher dimensional operators that are suppressed by the heavy mass scale of the physics that we integrated out. The effect of the UV physics to our low-energy theory is encoded in the couplings of these operators.

If the fundamental theory is not known then one can employ Weinberg's theorem [82], which states that the process described above is equivalent to powercounting subleading operators to the leading order action. As long as one writes down the most general set of operators consistent with the symmetries of the full theory, then it should yield the same Green's functions as the fundamental theory.

This can be expressed as an energy expansion where the expansion parameter is given by $E / \Lambda$, with $E$ being the energy of our low-energy system and $\Lambda$ being a heavy mass scale. The effective Lagrangian is given by the leading order terms plus an expansion of the form

$$
\mathcal{L}_{E f f}=\mathcal{L}[\psi]+\sum c_{i} \frac{O_{i}[\psi]}{\Lambda^{d_{i}-4}},
$$

where $c_{1}$ are the dimensionless constant coefficients whose strengths are determined by the couplings of the full theory and $O_{i}[\psi]$ are local operators of dimension $d_{i}$. The operators that dominate in the IR (infrared scales) are known as relevant operators and have dimension less than four, while operators that dominate in the UV (ultraviolet scales) are known as irrelevant and have dimension larger than four. Finally, operators with dimension equal to four are called marginal operators. In this work we are interested on irrelevant operators which appear as small corrections to the leading order action in the IR. Although the sum in (3.1.1) is infinite, in practise only a few terms are needed to reach our current experimental accuracy. Irrelevant operators of very high dimension are usually suppressed by large powers of $\Lambda$ and are not experimentally accessible. For this reason, although EFTs go under the category of non-renormalizable theories, this is not a problem because they are finite. That is, in a finite expansion only a few parameters will have to be determined. These should be estimated by using observations.

When we construct effective descriptions in a cosmological background, the background geometry of spacetime is treated classically and only the perturbations are 
quantized. The energy expansion ceases to be valid when the energy ratio $E / \Lambda$ reaches order-one. This is when interaction terms become important and the effective description breaks down. The theory is said to be strongly coupled as this is the energy scale at which new particles are produced. To put this into a Feynman language, the strong coupling scale is where loop diagrams are the same size as tree diagrams (i.e. the energy scale at which the loop expansion breaks down and perturbative unitarity in graviton scattering is violated).

The scale $\Lambda$ can be equivalent to the Planck scale or smaller but not arbitrarily small, i.e. the energy expansion makes sense only for as long as $E / \Lambda \ll 1$. In the bottom-up approach for single-field inflation we do not have knowledge of the explicit UV completion of our system, so instead we assume that heavy modes have been integrated out meaning that we can only trust the effective description at horizon crossing where the energy scale of inflation is given by $H \ll \Lambda$. Therefore for energies below the scale $\Lambda$, we treat this as a weakly coupled system and can safely assume that the vacuum state is given by Bunch-Davies.

\subsection{Extended theories of gravity}

Modified theories of gravity were developed to explore physics beyond the standard model of cosmology $(\Lambda \mathrm{CDM})$. They offer a rich phenomenology for dark energy which is otherwise identified with a very small and otherwise fine-tuned value for the cosmological constant. They are also ideal for studying deviations from the canonical inflation paradigm, where a scalar field is thought to be responsible for the accelerated expansion of the early universe. Modified theories of gravity fall under the more general category of extended theories of gravity, whose prerequisite is to relax the assumptions governing Lovelock's theorem [83,84] which states that only Einstein's equations plus a cosmological constant have second-order equations of motion in four-dimensions, i.e. the Lagrangian is taken to depend solely on the metric $\mathcal{L}\left[g_{\mu \nu}\right]$. The most usual extensions to gravity come by introducing a scalar field that is minimally or non-minimally coupled to gravity or by adding higher derivative corrections to the action.

In particular, in Scalar-Tensor theories the geometry of spacetime is affected not only by the curvature of spacetime, which is the usual case in Einstein gravity, but also by an additional scalar field. In that sense, the Lagrangian is taken to depend on both the metric and the scalar field $\mathcal{L}\left[g_{\mu \nu}, \phi\right]$. During the inflationary epoch, where scalar fields dominate the dynamics, matter fields are neglected all together. In the case where the scalar field is non-minimally coupled to curvature, the effect of the scalar degree of freedom is to alter the strength of the gravitational constant, so that the gravitational action acquires a time-dependent Planck mass, i.e. a modified Newton's constant, such as

$$
\frac{M_{P l}^{2}}{2} \int \mathrm{d}^{4} x f(\phi) R=\frac{1}{2} \int \mathrm{d}^{4} x \tilde{M} R, \quad \tilde{M}=M_{P l}^{2} f(\phi) .
$$

The Horndeski theories fall under the category of extended Scalar-Tensor theories. 
The action contains scalar-tensor modifications to gravity which lead to at most second-order equations of motion.

The Horndeski action was rediscovered through the Galileon theory which was originally formulated on flat spacetime [85], where the scalar field (or Galileon) enjoys a shift symmetry $\phi \rightarrow \phi+b_{\mu} x^{\mu}+c$ analogous to the classical Galilean transformations and where $b^{\mu}$ and $c$ are vector and scalar constants. Schematically, the Galileon Lagrangian is given by

$$
\mathcal{L}_{\text {Galil }} \sim \sum_{n=2}^{5} c_{n} \phi \mathcal{E} \mathcal{E}(\partial \partial \phi)^{n-1} \eta^{5-n}
$$

where $\mathcal{E}$ is the Levi-Civita symbol, $\eta$ represents the Minkowski metric and the contraction of indices is omitted. The Galileon effective description is well defined for as long as $\partial^{n} \phi / \Lambda^{n+1} \ll 1$, with $n \geq 3$.

The Galileon theory was later on generalized to curved spacetime but at the cost of breaking the Galilean symmetry. This is know as the covariant Galileon theory $[86,87]$, in which one promotes the Minkowski metric to $g_{\mu \nu}$ and partial derivatives to covariant derivatives. The latter can introduce additional curvature terms in the field equations, through their commutation relations (i.e. by application of the Ricci identity), which can be amended by adding suitable counterterms which help to realize second-order equations of motion. This can be extended further to the generalized Galileon theories which correspond to the most general covariant ScalarTensor setup of operators with second-order equations of motion. In this way the Horndeski theory [88] was rediscovered, albeit starting from different assumptions.

The most general model is known as Generalized inflation (G-inflation for short) [36] which can be used to realize models of canonical single-field inflation as well several extensions of the canonical inflationary paradigm, some of which are nonminimally coupled models [89], kinetically driven inflation (k-inflation) [90, 91], Dirac-Born-Infeld (DBI) inflation [92], $R^{2}$ inflation and many more.

Below we quickly review the key ingredients of G-inflation which we will employ throughout this work. This analysis was done in [36]. One can construct phenomenologically viable inflation models by starting from the following action

$$
S=\sum_{i=2}^{5} \int \mathrm{d}^{4} x \sqrt{-g} \mathcal{L}_{i}(\phi, X),
$$

where the Lagrangians are given by

$$
\begin{aligned}
& \mathcal{L}_{2}=G_{2}(\phi, X), \\
& \mathcal{L}_{3}=-G 3(\phi, X), \\
& \mathcal{L}_{4}=G_{4}(\phi, X) R+G_{4 X}\left[(\square \phi)^{2}-\left(\nabla_{\mu} \nabla_{\nu} \phi\right)^{2}\right], \\
& \mathcal{L}_{5}=G_{5}(\phi, X) G_{\mu \nu} \nabla^{\mu} \nabla^{\nu} \phi-\frac{G_{5 X}}{6}\left[(\square \phi)^{3}-3(\square \phi)\left(\nabla_{\mu} \nabla_{\nu} \phi\right)^{2}+2\left(\nabla_{\mu} \nabla_{\nu} \phi\right)^{3}\right],
\end{aligned}
$$


and

$$
X=-\frac{1}{2} \partial_{\mu} \phi \partial^{\mu} \phi
$$

Here, $G_{2}, G_{3}, G_{4}, G_{5}$ are generic functions of the scalar field and its first derivatives. We have adopted the notation $G_{\phi}=\partial G / \partial \phi$ and $G_{X}=\partial G / \partial X$. Standard inflation is described by the following choices $G_{2}=X-V(\phi), G_{4}=\frac{1}{2}, G_{3}=G_{5}=0$, where $V(\phi)$ is the inflaton potential and for simplicity $M_{P l}=1$. It is easy to see that in this case $\mathcal{L}_{4}$ corresponds to the Einstein-Hilbert term.

In this work we are interested only in kinetically driven inflationary models, therefore we take the functions $G_{a}, a=2, \cdots, 5$ to depend only on $X$. Working with a homogeneous scalar field $\phi=\phi(t)$ in the comoving gauge $\delta \phi=0$, one can obtain the background and constraint equations, in FRW spacetime, from the Lagrangian in (3.2.3). The field equations are given by

$$
\sum_{i=2}^{5} \mathcal{E}_{i}=\dot{\phi} J-G_{2}+2 X\left(6 H^{2} G_{4 X}+2 H^{3} \dot{\phi} G_{5 X}\right)-6 H^{2} G_{4}=0,
$$

and

$$
\sum_{i=2}^{5}\left(\mathcal{E}_{i}+\mathcal{P}_{i}\right)=\dot{\phi} J-2 X \ddot{\phi} G_{3 X}+2 \frac{\mathrm{d}}{\mathrm{d} t}\left[2 H\left(G_{4}-2 X G_{4 X}\right)-H^{2} X \dot{\phi} G_{5 X}\right]=0,
$$

where

$$
J=\dot{\phi}\left[G_{2 X}+3 H \dot{\phi} G_{3 X}+6 H^{2}\left(G_{4 X}+2 X G_{4 X X}\right)+H^{3} \dot{\phi}\left(3 G_{5 X}+2 X G_{5 X X}\right)\right] .
$$

Here we employ the ADM formalism [93], which we do not review. The contributions to the constraint equation (energy constraint) $\mathcal{E}_{i}$ are produced by variation of (3.2.3) with respect to $\mathrm{N}(\mathrm{t})$, where the metric is define as $\mathrm{d} s^{2}=-N^{2}(t) \mathrm{d} t^{2}+a^{2}(t) \mathrm{d} x^{2}$. The contributions to the evolution equation (pressure constraint) $\mathcal{P}_{i}$ are produced by variation with respect to $a(t)$. Finally, the generalized Friedmann equations given above are combined with the scalar-field equation, from which one gets

$$
\frac{1}{a^{3}} \frac{\mathrm{d}}{\mathrm{d} t}\left(a^{3} J\right)=0
$$

One can obtain the tensor action, expanded to second-order in metric perturbations, which is expressed as 


$$
S^{(2)}=\frac{1}{8} \int \mathrm{d} t \mathrm{~d}^{3} x a^{3}\left[\mathcal{G}_{T} \dot{h}_{i j}^{2}-\frac{\mathcal{F}_{T}}{a^{2}}\left(\nabla h_{i j}\right)^{2}\right]
$$

where $\mathcal{G}_{T}$ and $\mathcal{F}_{T}$ are defined as

$$
\begin{aligned}
& \mathcal{F}_{T}=2\left[G_{4}-X\left(\ddot{\phi} G_{5 X}+G_{5 \phi}\right)\right] \\
& \mathcal{G}_{T}=2\left[G_{4}-2 X G_{4 X}-X\left(H \dot{\phi} G_{5 X}-G_{5 \phi}\right)\right] .
\end{aligned}
$$

This introduces corrections to the kinetic term, whose overall effect is to scale the spatial coordinates with respect to the time coordinates. This leads to tensor fields propagating with a non-luminal speed

$$
c_{T}^{2}=\frac{\mathcal{F}_{T}}{\mathcal{G}_{T}}
$$

We demand positive values for the tensor speed

$$
c_{T}^{2}>0, \quad \text { with } \quad \mathcal{F}_{T}>0, \quad \mathcal{G}_{T}>0
$$

to ensure no gradient instability or else known as Laplacian instability. This is when a system becomes classically unstable against fluctuations of the background energy density. Demanding positive values for $\mathcal{F}_{T}, \mathcal{G}_{T}$ ensures correct signs for the kinetic term so to avoid ghost instabilities. Furthermore, causality considerations imply that perturbations of the fields should propagate with at most luminal speeds. The form of the action (3.2.10) was derived in [36].

At superhorizon scales this has solutions

$$
h_{i j}(\mathbf{x}, t) \simeq C_{i j}(\mathbf{x})+D_{i j}(\mathbf{x}) \int \frac{\mathrm{d} t^{\prime}}{a^{3}\left(t^{\prime}\right) \mathcal{G}_{T}\left(t^{\prime}\right)},
$$

where $C_{i j}, D_{i j}$ come from integrating the gravitational wave equations. It is customary to assume slow-varying functions which can be approximated as $\mathcal{G}_{T} \simeq$ const. [36]. In this case, the second term in (3.2.14), also known as the decaying mode, becomes negligible and can be ignored. However, there are cases in which $\mathcal{G}_{T}$ can be taken to be a rapidly varying function of time resulting into the second-term in (3.2.14) behaving as a growing mode [8]. This case will be discussed later in the text.

In the case where $\mathcal{F}_{T}, \mathcal{G}_{T}$ are taken to be slow-varying functions of time the tensor powerspectum is found to be [36]

$$
P_{h}=\frac{2 H^{2}}{4 \pi^{2} c_{T} \mathcal{F}_{T}}
$$


Note that the speed of propagation for primordial gravitational waves is still largely unconstrained. Next we look at the scalar sector. The action for scalar-fluctuations is given by

$$
S^{(2)}=\frac{1}{2} \int \mathrm{d} t \mathrm{~d}^{3} x a^{3}\left[\mathcal{G}_{S} \dot{\mathcal{R}}^{2}-\frac{\mathcal{F}_{S}}{a^{2}}(\nabla \mathcal{R})^{2}\right]
$$

where $\mathcal{G}_{S}$ and $\mathcal{F}_{S}$ are defined as

$$
\begin{aligned}
\mathcal{F}_{S} & =\frac{\Sigma}{\Theta^{2}} \mathcal{G}_{T}^{2}+3 \mathcal{G}_{T}, \\
\mathcal{G}_{S} & =\frac{1}{a} \frac{\mathrm{d}}{\mathrm{d} t}\left(\frac{a}{\Theta} \mathcal{G}_{T}^{2}\right)-\mathcal{F}_{T},
\end{aligned}
$$

where $\Sigma$ and $\Theta$ are expressed in terms of the functions $G_{a}(X)$ and are given by

$$
\begin{aligned}
\Sigma & =X G_{2 X}+2 X^{2} G_{2 X X}+12 H \dot{\phi} X G_{3 X}+6 H \dot{\phi} X^{2} G_{3 X X}-6 H^{2} G_{4} \\
& +6 H^{2}\left(7 X G_{4 X}+16 X^{2} G_{4 X X}+4 X^{3} G_{4 X X X}\right) \\
& +30 H^{3} \dot{\phi} X G_{5 X}+26 H^{3} \dot{\phi} X^{2} G_{5 X X}+4 H^{3} \dot{\phi} X^{3} G_{5 X X X}
\end{aligned}
$$

and

$$
\begin{aligned}
\Theta & =-\dot{\phi} X G_{3 X}+2 H G_{4}-8 H X G_{4 X}-8 H X^{2} G_{4 X X} \\
& -H^{2} \dot{\phi}\left(5 X G_{5 X}+2 X^{2} G_{5 X X}\right) .
\end{aligned}
$$

For recent pedagogical reviews, on Horndeski theories and beyond, see [94]. For a more comprehensive review on modified theories of gravity, we point the reader to $[95]$.

Finally, there are several theories that go beyond the canonical paradigm such as $P(X)$ theories which involve only single derivatives of the scalar field with $X=$ $-(\partial \phi)^{2} / \Lambda^{4}$ and $\Lambda$ being the strong coupling scale. These have been used to examine kinetic-inflation models, i.e. see [90,91]. Additionally, there are the so called DBI (Dirac-Born-Infield) theories $\mathcal{L}_{D B I} \sim-\Lambda^{4} \sqrt{1-X}$ where the scalar field enjoys a non-linearly realized symmetry $\phi(x) \rightarrow \phi(x)+b_{\mu} x^{\mu}+\phi(x) b^{\mu} \partial_{\mu} \phi(x) / \Lambda^{4}$, with $x=x^{\mu}$, which derive from the five-dimensional Poincaré invariance $[70,96]$. These type of inflationary theories are known to produce large non-Gaussianity in the scalar sector.

There is, indeed, a large family of theories that go beyond Einstein gravity with a wide spectrum of observational consequences for both inflation and theories of the late-time acceleration of the Universe (Dark Energy). In this work we use modifications to gravity to find new observational signatures for gravitational waves from inflation. 


\section{Chiral primordial gravitational waves in extended theories of Scalar- Tensor gravity}

\subsection{Abstract}

We re-examine the problem of parity violation in single field inflation. We look for a systematic way to parametrically approach the scale at which maximal parity violation occurs, which is where we expect to find the presence of the Chern Simons instability. We do so by considering possible realizations of the effective field theory of Scalar-Tensor gravity, which could offer a rich phenomenology. The gravitational action is extended to include derivatively coupled interactions which, by means of a disformal transformation, are scaled by negative powers of a small parameter which is identified with the graviton speed. This results in suppressing the cutoff scale of the effective theory leading to parametrically large chiral tensor fluctuations. We conclude that a change in the physical description of the system is necessary in order to maintain sufficient parity violation as well ensure stability of the modes.

\subsection{Introduction}

In Einstein's theory of General Relativity parity is conserved. On the other hand, extensions to gravity, motivated by high energy physics, require the addition of parity violating terms to the Einstein-Hilbert action [97]. These corrections create a difference in the intensities of the left and right gravitational wave polarizations resulting to a net circular polarization in the gravitational wave background.

It has been shown that if such an asymmetry was generated during inflation it could leave an observable trace in the cosmic microwave background (CMB), i.e. by producing non-vanishing TB and EB mode correlations [98-101], whose amplitude is characterized by the scalar-tensor ratio $r$ and the degree of polarization $\Pi$. The latter is defined as the difference between the tensor power spectra of left- and right-helicity modes, at the end of inflation, normalized by the total amplitude

$$
\Pi=\frac{\mathcal{P}_{h}^{L}-\mathcal{P}_{h}^{R}}{\mathcal{P}_{h}^{L}+\mathcal{P}_{h}^{R}}, \quad-1<\Pi<1
$$


and it can take values between -1 and 1 for maximally right or left-handed signal or zero when circular polarization is completely absent from the gravitational wave background.

Future experiments, such as SPIDER, CMBpol and LiteBIRD, will measure the B-Mode polarization anisotropies with expected precision $r \sim 10^{-3}[10,11,102$, 103]. Additionally, a direct detection of a primordial circularly polarized stochastic gravitational wave background may be possible with space-based interferometers, such as LISA, DECIGO (Deci-Hertz) or their proposed successor BBO (Big Bang Observer) [6,12,13,104-106], as well with PTAs (Pulsar Timing Arrays) [14].

It has been argued that it, ideally, requires a large circular polarization degree $\Pi$ in order to make a detection of parity violation $[99,107,108]$ (for a more recent summary see [109]). Unfortunately, theoretical predictions for single field inflation tend to suffer quantitatively due to the Chern Simons instability [16,110-114], leading to a negligible chirality enhancement.

Alternative approaches have been to consider two-field inflationary models [111], by introducing a curvaton or several gauge fields [114], couplings in term of a massive field [115] or to consider models of inflation involving non-Abelian gauge fields [116]. The latter was an extension of the work in [117] where parity violating tensor perturbations, during inflation, were used to build a leptogenesis model in which they focused on the short distance modes. This model was further extended in [118] to examine baryogenesis in the dark sector. Other approaches have been to study gravitational wave polarisation in Horava-Lifshitz gravity [119,120]. See also [121] for a top-down approach.

In this work we re-examine the problem of parity violation in the context of single field inflation. The generic effective field theory (EFT) for single field inflation was studied by Weinberg in [80] in which they produced the most general set of operators that contribute to the lower energy physics. The gravitational action was extended at next-to-leading order (NLO) to include the Weyl squared tensor and the gravitational Chern Simons term, with the latter being responsible for parity violation. There it was shown that for the energy expansion to be finite ${ }^{1}$ the heavy mass scale $\Lambda$ suppressing the higher-derivative operators cannot be much smaller than the reduced Planck mass $M_{P l}$. Considering that $M_{P l} \simeq 10^{18} \mathrm{GeV}$ and the energy scale of inflation is constrained to be $H \lesssim 10^{13} \mathrm{GeV}$ [15] one would expect parity violating effects to be very small. Therefore, different assumptions are needed if one wishes to examine a regime where new physics are expected to be much closer to the scales that we can measure in the CMB.

In this work we aim to approach this regime parametrically. We ideally want to keep the heavy mass scale $\Lambda \simeq M_{P l}$ fixed and instead introduce a parameter whose effect will be to parametrically suppress the value of $\Lambda$. The most straightforward way to achieve this is by introducing a non-trivial dispersion relation. This process is well understood in, for example, the effective field theory of inflation (EFTI) studied by Cheung et al. [122]. There it is the broken time diffeomorphism (Stückelberg trick) which introduces extra pieces to the action. This, naturally, results to a non-

\footnotetext{
${ }^{1}$ This is a generic effective field theory where various coefficients are assumed to be of order unity and the strength of the higher-order corrections is determined by dimensional analyses.
} 


\section{CHIRAL PRIMORDIAL GRAVITATIONAL WAVES IN EXTENDED THEORIES OF SCALAR-TENSOR GRAVITY}

trivial speed for the scalar and tensor sectors whose effect is to suppress the heavy mass scale of the non-quadratic fluctuations resulting to the strong coupling scale of the theory appearing parametrically below the scale at which the background was integrated out, leading to large scalar non-Gaussianity. Later on, they addressed this issue in $[123,124]$ by studying weakly coupled completions of the EFTI action for scalar fluctuations. $P(X)$ theories, where $X=-1 / 2 \partial_{\mu} \phi \partial^{\mu} \phi$, are also known to produce a similar scenario in the non-relativistic limit [125].

Here we approach this from the point of view of Horndeski theories (or beyond) which are known to be characterized by non-trivial dispersion relations. Assuming there exists a UV completion for the Horndeski theory, one could take a bottomup approach in which the Horndeski theory could be extended, from an effective field theory point of view, by writing down the most general set of operators that agree with the symmetries and particle content of the full theory. So far only partial extensions to parity-preserving Scalar-Tensor theories have been attempted [126,127] while ghost-free parity violating corrections to Scalar-Tensor theories were separately examined in [128].

Here we produce an extension to the Horndeski theory (or beyond) by employing a disformal transformation of the metric. It is well known that disformal transformations can generate Horndeski or beyond Horndeski theories. Special disformal transformations, where the disformal function depends only on the scalar field $\phi$, were analysed in [129]. General disformal mappings of the Einstein-Hilbert action were considered in [130], while general disformal transformations of quadratic DHOST Lagrangians were investigated in $[131,132]$. There it was shown that disformal transformations introduce extra pieces to the action which, naturally, change the dispersion relation for gravitons.

We extend on these ideas by including disformally transformed higher-curvature operators from an EFT point of view. As one expects, the formulations can quickly grow to be too cumbersome when working with disformal transformations at the covariant level. While we have already taken steps towards that direction (as an example see Appendix A.11), it is convenient to first examine disformal transformations of higher-curvature operators for cosmological perturbations as they are simpler. The results of this work are to be understood as indicative of what can be achieved when one considers chiral scalar-tensor extensions to Horndeski's theory and beyond.

The aim of this work is to parametrically suppress the heavy mass scale $\Lambda$ of the higher-derivative operators. To examine this in a systematic way and in the simplest way possible, we employ an inverse disformal transformation [133] on the extended action for tensors in [80]. We find that if the higher-curvature operators are scaled by inverse powers of a small parameter $c_{T}$, which we identify with the graviton speed, this pushes their contributions into the "UV sensitive" regime, where the energy scale suppressing these corrections is well below the scale of the reduced Planck mass $M_{P l}$, leading to parametrically large chiral tensor fluctuations. In this sense the effective field theory is organized into an energy expansion, where $E / \Lambda_{*}$ is the expansion parameter and $\Lambda_{*}$ is an effective mass scale which parametrically depends on some power of $c_{T}$. We find maximal parity violation occurs for parametrically 
small values of $c_{T} \ll 1$ in the limit that $E^{2} / \Lambda_{*}^{2} \rightarrow 1$. This signals the breakdown of the perturbative expansion and the presence of the Chern Simons instability. Furthermore we expect cubic or higher-order interactions to become important resulting to a strongly coupled theory.

We conclude that a change in the physical description of the system is necessary in order to maintain sufficient parity violation as well ensure stability of the theory. Preliminary results on cubic interactions indicate that one would ideally need to work with a weakly coupled completion of our system. Such completions have already been attempted in the scalar sector of the effective field theory of inflation (EFTI) studied by Cheung et al. [123,124].

The paper is organized as follows. In Section 4.3 we introduce the quadratic action which we disformally transform in Section 4.3.1. We then examine the validity of the EFT in Section 4.3.2 and proceed to produce second-order equations of motion in Section 4.3.3. Next, we identify the conditions for a stable Chern-Simons theory in Section 4.4 and proceed to solve the linearised theory in the simplest way possible in Section 4.5. Finally we evaluate the power spectrum in Section 4.5.1 and briefly look at the different representations of the theory in Section 4.6. We conclude our results in Section 4.7 .

\subsection{Parametric amplification of chiral primordial gravitational waves}

We consider possible realizations of the effective field theory of Scalar-Tensor gravity which could offer a rich phenomenology. One can start with a gravity + scalar system and extend it with higher-derivative operators from an EFT point of view. Here we achieve this with an inverse disformal transformation of the quadratic action. We take a bottom-up approach, in which the action is organized as an energy expansion, where the leading-order Lagrangian is that of standard canonical Einstein gravity, while the higher-order Lagrangians are treated perturbatively. At next-to-leading order (NLO), i.e. four-derivative operators, the action reads [80]

$$
S^{(0)}=\frac{M_{P l}^{2}}{2} \int \mathrm{d}^{4} x \sqrt{-g}\left\{R+\frac{f_{1}}{M_{P l}^{2}} W^{\mu \nu \rho \sigma} W_{\mu \nu \rho \sigma}+\varepsilon^{\mu \nu \rho \sigma} \frac{f_{2}}{M_{P l}^{2}} W_{\mu \nu \kappa \lambda} W_{\rho \sigma}^{\kappa \lambda}\right\}
$$

where $W_{\mu \nu \rho \sigma}$ is the Weyl tensor, and $\varepsilon^{\mu \nu \rho \sigma}=(-g)^{-\frac{1}{2}} \epsilon^{\mu \nu \rho \sigma}$ is the totally antisymmetric Levi-Civita tensor density. The reduced Planck mass is defined as $M_{P l}^{-1}=\sqrt{8 \pi G}$, with $G$ being the gravitational Newton's constant. The last term in (4.3.1) is the gravitational Chern Simons term, which is sometimes denoted as $W \tilde{W}$ and it is responsible for parity violation.

We denote with $\Lambda \simeq M_{P l}$ the energy scale of the heavy degrees of freedom that have been integrated out of the Lagrangian. Such high energy processes are not experimentally accessible to us, but instead they enter the low energy action, orderby-order, though the coefficients in the derivative expansion. Therefore, higher-order 


\section{CHIRAL PRIMORDIAL GRAVITATIONAL WAVES IN EXTENDED THEORIES OF SCALAR-TENSOR GRAVITY}

corrections are expected to be subdominant as they are suppressed by the heavy mass scale $\Lambda$ which is the energy scale at which we expect to find "new physics". For a great review on EFTs see $[81,134]$ and the excellent reviews by C.P. Burgess.

The functions $f_{1}(\phi)$ and $f_{2}(\phi)$ represent generic couplings of the dimensionless scalar field $\phi=\phi_{c} / \Lambda \simeq \phi_{c} / M_{P l}$ which satisfies inflationary dynamics and is homogeneous and isotropic. In the absence of a UV complete description, the form of the coupling strengths can be motivated from phenomenological considerations and/or experimental observations.

From now on we choose to work with the conformal time $\eta$ and assume an isotropic and homogeneous FRW cosmology with line element

$$
\mathrm{d} s^{2}=a(\eta)^{2}\left[-\mathrm{d} \eta^{2}+\mathrm{d} x^{2}\right]
$$

where $a=-(H \eta)^{-1}$ is the scale factor and take the scalar field to be homogeneous $\phi=\phi(t)$. Although, during inflation, the de Sitter symmetries are taken to be broken we choose, for simplicity, to work in an approximately exact de Sitter space with the Hubble parameter given by $H(t) \sim H=$ const. and where $t$ is the proper cosmic time. Finally, the metric is expanded, up to second-order in perturbations, around a de Sitter background

$$
g_{\mu \nu}=\tilde{g}_{\mu \nu}+h_{\mu \nu}, \quad \frac{h_{\mu \nu}}{g_{\mu \nu}} \ll 1
$$

where the perturbations respect the transverse-traceless (TT) conditions, namely $\partial_{i} h_{j}^{i}=h_{i}^{i}=0$. In what follows we focus only on the tensor sector.

\subsubsection{Disformally transformed action}

We choose to express the quadratic action in terms of barred parameters (see Appendix A.9). Therefore, we consider the effects of the inverse of a disformal transformation of the form

$$
g_{\mu \nu} \rightarrow \bar{g}_{\mu \nu}=c_{T}\left[g_{\mu \nu}+\left(1-c_{T}^{2}\right) n_{\mu} n_{\nu}\right]
$$

Here we have used the normalization $n_{\mu}=\phi_{, \mu} / \sqrt{2 X}$ with $n_{\mu} n^{\mu}=-1$ and $X=$ $-\frac{1}{2} \phi_{, \mu} \phi^{\mu}$. We follow the methods in $[133,135,136]$ where it was shown that in an FRW cosmological setting, with the scalar field $\phi$ taken to be homogeneous, a disformal transformation corresponds to a redefinition of the time-coordinate and the scale factor. In particular, for an inverse disformal transformation, one can make the following redefinitions

$$
\mathrm{d} \bar{\eta}=c_{T} \mathrm{~d} \eta, \quad \bar{a}=c_{T}^{\frac{1}{2}} a
$$


where for simplicity we take the disformal parameter $c_{T}=$ const. and $\bar{f}_{1}, \bar{f}_{2}$ to be functions of the conformal time $\bar{\eta}$. At second-order in perturbations of the metric ${ }^{2}$ the contributions to (4.3.1) transform as follows (see Appendix A.9 for more general expressions)

$$
\begin{aligned}
S^{(2)} & =\frac{M_{P l}^{2}}{2} \int \mathrm{d}^{4} x\left\{\frac{a^{2}}{4}\left[\left(h_{i j}^{\prime}\right)^{2}-c_{T}^{2}\left(\nabla h_{i j}\right)^{2}\right]+\epsilon^{i j k 0} \frac{8 \bar{f}_{2}}{c_{T}^{2} M_{P l}^{2}} \partial_{i} h_{l j}^{\prime}\left[\mathcal{H} h_{l k}^{\prime}+h_{l k}^{\prime \prime}\right]\right. \\
& \left.+\frac{\bar{f}_{1}}{M_{P l}^{2}}\left[\frac{\left(h_{i j}^{\prime \prime}\right)^{2}}{2 c_{T}^{3}}+\frac{h_{i j}^{\prime \prime} \nabla^{2} h_{i j}}{c_{T}}+\frac{c_{T}\left(\nabla^{2} h_{i j}\right)^{2}}{2}-\frac{2\left(\nabla h_{i j}^{\prime}\right)^{2}}{c_{T}}\right]\right\},
\end{aligned}
$$

where we denote time derivatives with a prime $\left(\partial_{\eta}=^{\prime}\right)$. The Hubble parameter in terms of the conformal time is given by $\mathcal{H}=a H$ and we use Latin indices to denote spatial components. At leading order in (4.3.6) we find a quadratic action with gravitons having a non-trivial speed, given by

$$
S_{L O}^{(2)}=\frac{M_{P l}^{2}}{2} \int \mathrm{d}^{4} x \frac{a^{2}}{4}\left[\left(h_{i j}^{\prime}\right)^{2}-c_{T}^{2}\left(\nabla h_{i j}\right)^{2}\right]
$$

This is because, at the level of the perturbations, the effect of a disformal transformation can be seen as a stretching of the time-coordinate with respect to the spatial coordinate. This can also be understood by considering the effect of a disformal transformation at the covariant level. For example, in [130] (see Appendix B.1, relation (B10) in [130]) it was shown that the effect of a pure disformal transformation to the Einstein-Hilbert action translates to adding extra pieces to the action which results in producing a Horndeski like theory. Such theories are known to be characterised by non-trivial dispersion relations. In this sense, the next natural step is to identify the parameter $c_{T}$ in (4.3.6) to be the leading order contribution to the tensor speed. Similarly, the higher-curvature contributions are modified by acquiring extra pieces (as an example see Appendix A.11).

It is interesting to note that the leading order action in (4.3.7) is related to the quadratic action for tensors in [122], namely

$$
S^{(2)}=\frac{M_{P l}^{2}}{2} \int \mathrm{d}^{4} x \frac{a^{2}}{4} c_{T}^{-2}\left[\left(h_{i j}^{\prime}\right)^{2}-c_{T}^{2}\left(\nabla h_{i j}\right)^{2}\right]=\frac{\tilde{M}_{P l}^{2}}{2} \int \mathrm{d}^{4} x \frac{a^{2}}{4}\left[\left(h_{i j}^{\prime}\right)^{2}-c_{T}^{2}\left(\nabla h_{i j}\right)^{2}\right],
$$

by a conformal transformation which can be used to set the modified Planck mass $\tilde{M}_{P l}$ in (4.3.8) to standard. In [122] it is a broken time diffeomorphism (Stückelberg trick) which introduces extra pieces to the action. This naturally results to a nontrivial speed for gravitons. It would be interesting to see how parity violation is affected in their setup. In their work they focused on curvature perturbations which

\footnotetext{
${ }^{2}$ Here we used the formulations in [137] and the Mathematica package in [138] to produce the perturbed expressions.
} 


\section{CHIRAL PRIMORDIAL GRAVITATIONAL WAVES IN EXTENDED THEORIES OF SCALAR-TENSOR GRAVITY}

were treated as Goldstone boson modes. There it was found that a small scalar speed reduces the mass scale that suppresses the non-quadratic fluctuations leading to sizeable non-Gaussianity.

Here we find that, in a similar fashion, higher-order quadratic operators (and consequently non-quadratic operators) with more time-derivatives are enhanced, for small parameter $c_{T} \ll 1$, compared to operators with spatial derivatives ${ }^{3}$. In this way the sub-leading terms in (4.3.6) could become sizeable as they are scaled by negative powers of a small parameter, leading to parametrically large chiral tensor fluctuations. Next we look at the phenomenological consequences of our set-up.

\subsubsection{Validity of the EFT}

The main aim of this work is to parametrically approach the regime at which the EFT breaks down, which is where we expect the Chern Simons instability to appear in the system.

The effective action in (4.3.6) is organized into an energy expansion which is in terms of powers of the expansion parameter $E / \Lambda_{*}$, where $\Lambda_{*}$ is an effective mass scale proportional to some power of the parameter $c_{T}$. The energy expansion will continue to be valid until the sub-leading terms become as important as the leading order terms. Therefore we expect that the cosmological perturbation theory will break down in the non-relativistic limit $c_{T} \ll 1$, i.e our effective field theory will cease to be meaningful if the ratio $E / \Lambda_{*} \rightarrow 1$. Putting this into a Feynman language, at this point one would expect the propagator of the free field theory to pick up substantial contributions which can affect the leading order kinematics. This can also result into the presence of unphysical states in the system [80]. The usual prescription is that one will need to add extra pieces to the action in order to restore the validity of the $\mathrm{EFT}^{4}$.

To find when this happens we need to estimate the form of the effective mass scale $\Lambda_{*}$ by examining the action in (4.3.6) which is quadratic in the fields. The case where $c_{T}=1$ was considered in [139]. Here we wish to find the effective mass scale of (4.3.6) for $c_{T} \ll 1$. We employ the methods in [123] (see Section 2). We restore fake Lorentz invariance by defining the following rescaling of the spatial coordinates $x \rightarrow \tilde{x}=c_{T}^{-1} x$ and the canonically normalized tensor perturbation

$$
\tilde{h}_{i j}^{2}=\frac{c_{T}^{3} M_{P l}^{2} h_{i j}^{2}}{4}
$$

From this we find that the action in (4.3.6) becomes

\footnotetext{
${ }^{3} \mathrm{~A}$ similar conclusion was reached in [122] for the scalar sector. In their case it was the spatial derivatives that were enhanced with respect to time-derivatives.

${ }^{4}$ At some stage one may have to work with the UV complete description of the system.
} 


$$
\begin{aligned}
S^{(2)} & =\frac{1}{2} \int \mathrm{d} \eta \mathrm{d}^{3} \tilde{x}\left\{\left[\left(\tilde{h}_{i j}^{\prime}\right)^{2}-\left(\tilde{\nabla} \tilde{h}_{i j}\right)^{2}\right]+\epsilon^{i j k 0} \frac{32 \bar{f}_{2}}{c_{T}^{3} M_{P l}^{2}} \tilde{\partial}_{i} \tilde{h}_{l j}^{\prime}\left[\mathcal{H} \tilde{h}_{l k}^{\prime}+\tilde{h}_{l k}^{\prime \prime}\right]\right. \\
& \left.+\frac{\bar{f}_{1}}{c_{T}^{3} M_{P l}^{2}}\left[2\left(\tilde{h}_{i j}^{\prime \prime}\right)^{2}+4 \tilde{h}_{i j}^{\prime \prime} \tilde{\nabla}^{2} \tilde{h}_{i j}+2\left(\tilde{\nabla}^{2} \tilde{h}_{i j}\right)^{2}-8\left(\tilde{\nabla} \tilde{h}_{i j}^{\prime}\right)^{2}\right]\right\} .
\end{aligned}
$$

The effective mass scale of the theory $\Lambda_{*}^{2} \simeq c_{T}^{3} M_{P l}^{2}$ can be read off directly from (4.3.10), where for simplicity we have ignored the time-dependence in $\bar{f}_{1}$ and $\bar{f}_{2}$ and treat them as order-one parameters. To ensure the validity of low energy observables we need our perturbative expansion to hold at the relevant scales that we can measure in the CMB, i.e. at horizon crossing where the fluctuations freeze. Therefore, we demand that the size of the fluctuations at the de Sitter scale $k_{p h} \sim H$, where $k_{p h}=k / a$ is the physical momentum, coming from the higher-order corrections, is much less than $\mathcal{O}(1)$. Therefore, we have

$$
\frac{k_{p h}^{2}}{\Lambda_{*}^{2}} \simeq \frac{H^{2}}{c_{T}^{3} M_{P l}^{2}} \ll 1,
$$

which translates to a lower bound on the graviton speed.

Additionally, the need for a finite perturbative expansion may require us to include next-to-next-to-leading order (NNLO) operators, i.e. six-derivative terms, as they can pick up enhancements that could stand them relevant to the calculation. This is because at (NNLO) the energy expansion ratio will be of order $H^{4} / \Lambda_{*}^{4}$ (see Appendix A.12). Higher-derivative parity preserving extensions to scalar-tensor gravity were discussed in [126] while higher-derivative parity-violating operators for Scalar-Tensor chiral theories were discussed in [128], where alongside the gravitational Chern Simons term they included first- and second-derivatives of the scalar field. These were subsequently studied in [140]. We only tentatively look at these in Appendix A.12.

Additionally, in the limit $c_{T} \ll 1$ non-quadratic terms can become important i.e. terms of the form $\sim h h h$. Parity violation in tensor non-Gaussianity was investigated in $[137,141]$. They showed that there is no parity violation in de Sitter, but found non-vanishing contributions to the bispectrum when slow-roll inflation is taken into account. In particular the three-point correlators $\langle T T B\rangle\langle T E B\rangle\langle E E B\rangle$ could become non-vanishing, as opposed to the parity conserving case, which could be observed in the CMB data. Furthermore, mixed correlators were more recently studied in [142].

We do not consider in detail the effect of higher-order interaction terms in this work although it would be interesting to examine how the bispectrum is affected in a more realistic scenario of our setup. In terms of our arguments, we require that cubic interactions remain sub-leading. As an example, take a cubic interaction of the form ${ }^{5}$

\footnotetext{
${ }^{5}$ Such interaction terms can be found by expanding the Weyl squared tensor to third-order in perturbations of the metric.
} 


\section{CHIRAL PRIMORDIAL GRAVITATIONAL WAVES IN EXTENDED THEORIES OF SCALAR-TENSOR GRAVITY}

$$
S^{(3)}=\frac{M_{P l}^{2}}{2} \int \mathrm{d} \eta \mathrm{d}^{3} x \frac{f_{1}}{M_{P l}^{2}}\left[\frac{1}{c_{T}^{3}} \mathcal{H} h_{i j}^{\prime} h_{i l}^{\prime} h_{l j}^{\prime}-2 c_{T} h_{i j} \partial_{i} \partial_{j} h^{l m} \nabla^{2} h_{l m}+\cdots\right]
$$

Once we restore fake Lorentz invariance, we find

$$
S^{(3)}=\frac{1}{2} \int \mathrm{d} \eta \mathrm{d}^{3} x c_{T}^{-\frac{9}{2}} \frac{f_{1}}{M_{P l}^{3}}\left[8 \mathcal{H} h_{i j}^{\prime} h_{i l}^{\prime} h_{l j}^{\prime}-16 h_{i j} \partial_{i} \partial_{j} h^{l m} \nabla^{2} h_{l m}+\cdots\right] .
$$

From this we can see that the energy expansion ratio is of the order $E^{3} / \Lambda_{*}^{3}$. We can approximate the following constraint coming from demanding that the cubic interactions do not dominate at around the energy scale of inflation $k_{p h} \sim H$, which is

$$
\frac{k_{p h}^{3}}{\Lambda_{*}^{3}} \simeq c_{T}^{-\frac{9}{2}} \frac{H^{3}}{M_{P l}^{3}} \ll 1,
$$

We see that in the limit $c_{T} \ll 1$ new physics could appear at energies not far above the energy scale of inflation. Consequently, the higher-order interactions may acquire large couplings which could leave measurable evidence, from the new physics, in the CMB data. Furthermore, the Chern Simons instability will appear in our system for some $\mathcal{O}(1)$ value of (4.3.11) and (4.3.14) for which we find the limiting value

$$
c_{T} \simeq\left(\frac{H}{M_{P l}}\right)^{\frac{2}{3}} .
$$

We will reach the same conclusions when we examine the effective potential, later on in the text. The behaviour of the coefficients for the quadratic and cubic interactions around the limit (4.3.15) is shown in figure (4.1). From this we conclude that the Chern Simons instability is a consequence of the strongly coupled theory. This can be seen in the graph, as $c_{T}$ approaches the limit in (4.3.15) the cubic interactions (in blue) shoot up with respect to the quadratic interactions (in red) which, by definition, leads to the theory being strongly coupled.

\subsubsection{The equations of motion}

Before we begin with our analysis, we need to briefly discuss the Ostrogradsky instability whose no-go theorem $[143,144]$ is central in the study of higher-order corrections to gravity. The effective Lagrangian in (4.3.6) includes contributions that lead to higher than second-order equations of motion, which cannot always be removed by partial integration. While we cannot excite the Ostrogradsky ghost, as long as we remain in the low-energy regime of the EFT, the system can still exhibit unphysical effects if the equations of motion are higher than order-two ${ }^{6}$.

\footnotetext{
${ }^{6}$ It will require additional initial conditions in order to eliminate unwanted solutions. At best such systems can only be solved numerically.
} 


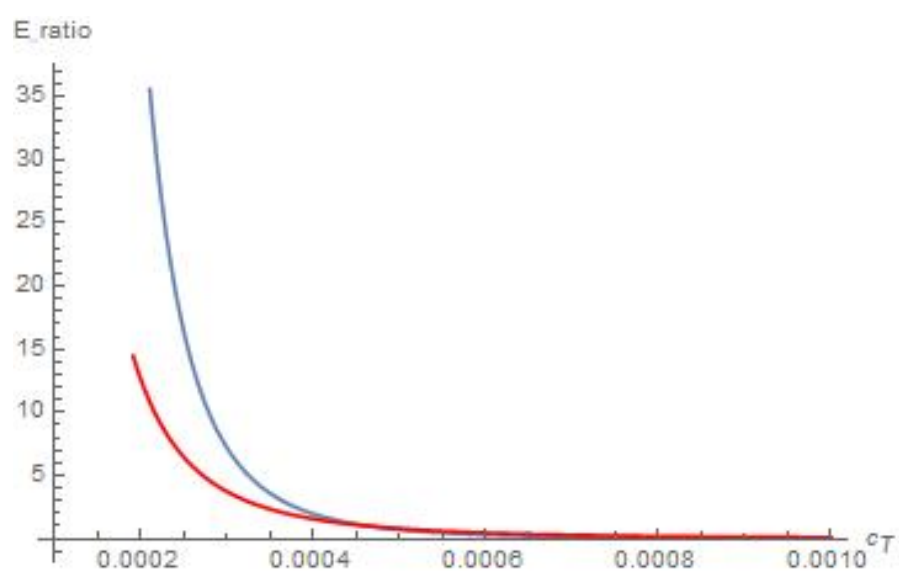

Figure 4.1: Once the parameter $c_{T}$ attains the value in (4.3.15) cubic interactions (in blue) become more important than the quadratic ones (in red), signalling the break down of the effective description. For this plot we take $M_{P l}=10^{18} \mathrm{GeV}$, and $H=10^{13} \mathrm{GeV}$. The horizontal axis displays the values of $c_{T}$ in the interval $\left\{10^{-4}, 10^{-3}\right\}$ which is the range in which we expect to find the value for $c_{T}$ in (4.3.15) for this particular numerical example, while the vertical axis displays the energy expansion ratio at the non-relativistic limit $E_{\text {ratio }} \sim \mathcal{O}(1)$.

For (NLO) contributions to the action it is possible to obtain second-order equations of motion via a field redefinition, which amounts to a substitution in terms of the equations of motion obtained from the leading order action in (4.3.7)

$$
h_{i j}^{\prime \prime}+2 \mathcal{H} h_{i j}^{\prime}-c_{T}^{2} \nabla^{2} h_{i j}=0 .
$$

This way one can shift the offending terms at higher-orders in the expansion. As one expects, this can complicate things when working at (NNLO). It is not, in general, trivial to find field redefinitions that ensure second-order equations of motion when the action contains a combination of (NLO) and (NNLO) operators. Although, such field redefinitions may be possible to find, they tend to be quite involved. As an example, we would like to point the reader to the analysis that was performed in [145] for the scalar sector. It would be interesting to see if, in the future, we could obtain something similar for tensors. As we do not wish to enter into lengthy discussions regarding these issues we refer the reader to [146-148] and references therein (see also [149] for a recent pedagogical treatment).

For these reasons, here we focus at (NLO) corrections to gravity and only briefly discuss (NNLO) operators in Appendix A.12. At second-order in perturbations of the metric, we expect the disformally transformed action to contain, schematically, the following type of contributions 


$$
\begin{aligned}
S^{(2)} & =\frac{M_{P l}^{2}}{8} \int \mathrm{d}^{3} x \mathrm{~d} \eta a^{2}\left\{(1+\alpha)\left(h_{i j}^{\prime}\right)^{2}-\left(c_{T}^{2}+\beta\right)\left(\nabla h_{i j}\right)^{2}-\gamma\left(\nabla h_{i j}^{\prime}\right)^{2}+\delta\left(\nabla^{2} h_{i j}\right)^{2}\right. \\
& \left.-\epsilon^{i j k}\left[\varepsilon\left(h_{i}^{q}\right)^{\prime}\left(\partial_{j} h_{k q}\right)^{\prime}-\zeta\left(\partial^{r} h_{i}^{q}\right) \partial_{j} \partial_{r} h_{k q}\right]\right\},
\end{aligned}
$$

where the coefficients $\alpha-\zeta$ are functions of the conformal time. This form of the action guarantees second-order equations of motion. Using (4.3.16) we find that the action in (4.3.6) takes the following form

$$
\begin{aligned}
S^{(2)} & =\frac{M_{P l}^{2}}{8} \int \mathrm{d}^{3} x \mathrm{~d} \eta a^{2}\left\{\left(1+\frac{\bar{f}_{1} \mathcal{H}^{2}}{c_{T}^{3} a^{2} M_{P l}^{2}}\right)\left(h_{i j}^{\prime}\right)^{2}-\left[c_{T}^{2}+\frac{\left(\bar{f}_{1}^{\prime} \mathcal{H}+\bar{f}_{1} \mathcal{H}^{\prime}\right)}{c_{T} a^{2} M_{P l}^{2}}\right]\left(\nabla h_{i j}\right)^{2}\right. \\
& \left.-\frac{\bar{f}_{1}}{c_{T} a^{2} M_{P l}^{2}}\left(\nabla h_{i j}^{\prime}\right)^{2}+\frac{\bar{f}_{1} c_{T}}{a^{2} M_{P l}^{2}}\left(\nabla^{2} h_{i j}\right)^{2}-\frac{\bar{f}_{2}^{\prime}}{a^{2} M_{P l}^{2}} \epsilon^{i j k}\left[\frac{1}{c_{T}^{2}} h_{q i}^{\prime} \partial_{j} h_{k q}^{\prime}-\left(\partial^{r} h^{q}{ }_{i}\right) \partial_{j} \partial_{r} h_{k q}\right]\right\},
\end{aligned}
$$

where we have absorbed numerical factors into the definitions of $\bar{f}_{1}$ and $\bar{f}_{2}$. It is easy to see that (4.3.18) has the same form with (4.3.17) which guarantees secondorder equations of motion. Next, we produce the Euler-Lagrange equations which are expressed in terms of the canonically normalized field $\mu^{5}=\left(M_{P l} / 2\right) h^{s} z^{s}$, as ${ }^{8}$ (see Appendix A.9)

$$
\left(\mu^{s}\right)^{\prime \prime}+\left[k^{2} \frac{\left(c_{T}^{2}+\frac{\left(\bar{f}_{1} \mathcal{H}^{\prime}+\bar{f}_{1}^{\prime} \mathcal{H}\right)}{c_{T} a^{2} M_{P l}^{2}}-\frac{c_{T} k^{2} \bar{f}_{1}}{a^{2} M_{P l}^{2}}-\frac{\lambda^{s} k \bar{f}_{2}^{\prime}}{a^{2} M_{P l}^{2}}\right)}{\left(1+\frac{\bar{f}_{1} \mathcal{H}^{2}}{c_{T}^{3} a^{2} M_{P l}^{2}}-\frac{k^{2} \bar{f}_{1}}{c_{T} a^{2} M_{P l}^{2}}-\frac{\lambda^{s} k \bar{f}_{2}^{\prime}}{c_{T}^{2} a^{2} M_{P l}^{2}}\right)}-\frac{\left(z_{k}^{s}\right)^{\prime \prime}}{z_{k}^{s}}\right] \mu^{s}=0
$$

where the effective potential is defined in terms of

$$
z^{s}=a \sqrt{1+\frac{\bar{f}_{1} \mathcal{H}^{2}}{c_{T}^{3} a^{2} M_{P l}^{2}}-\frac{k^{2} \bar{f}_{1}}{c_{T} a^{2} M_{P l}^{2}}-\frac{\lambda^{s} k \bar{f}_{2}^{\prime}}{c_{T}^{2} a^{2} M_{P l}^{2}}}
$$

The parameter $s=L, R$ is used to denote left and right graviton modes and we have defined $\lambda^{s}= \pm 1$. Finally, we have omitted summation over left and right modes. It is now evident that the effective potential depends on the polarization modes. This produces an asymmetry in the amplitude of the solutions for left and right modes which leads to a circularly polarized gravitational wave background [99]. Next we look at the Chern Simons instability.

\footnotetext{
${ }^{7}$ Here we use the conventions in [37].

${ }^{8}$ We have dropped, for brevity, the $\mathbf{k}, \eta$ dependencies.
} 


\subsection{On the stability of parity breaking theories}

Let us for a moment simplify our arguments by setting $c_{T}=1$ in (4.3.20). It is convenient to express the effective potential in terms of the Chern Simons and Weyl squared tensor dynamical mass-scales [16] by defining

$$
M_{c s}=\frac{a M_{P l}^{2}}{f_{2}^{\prime}}, \quad \text { and } \quad M_{w}^{2}=\frac{M_{P l}^{2}}{f_{1}},
$$

respectively, where $k_{p h}=k / a$ is the physical wavenumber and as we will soon find out, $M_{w}^{2}<0$. Therefore, (4.3.20) becomes

$$
z^{s}=a \sqrt{1+\frac{\mathcal{H}^{2}}{a^{2} M_{w}^{2}}-\frac{k_{p h}^{2}}{M_{w}^{2}}-\frac{k_{p h} \lambda^{s}}{M_{c s}}} .
$$

If we ignore the Weyl squared tensor contributions, for a moment, it is then straight forward to see that the linear theory will break down for $\left(z^{s}\right)^{2}=0$, i.e. at $k_{p h}=M_{c s}$. The amplitude of one of the helicity mode develops an instability which appears as a logarithmic divergence [110]. In what follows we derive a constraint that can ensure the stability of all modes within the regime of the validity of the EFT. For the stability of the solutions it requires

$$
\left(z^{s}\right)^{2}>0 \Rightarrow \frac{4}{M_{w}^{2}}+\frac{4 \mathcal{H}^{2}}{a^{2} M_{w}^{4}}<-\left(\frac{\lambda^{s}}{M_{c s}}\right)^{2} .
$$

The quantity on the RHS of the inequality is negative. This implies that the only way to satisfy this relationship is to demand that the Weyl squared dynamical mass scale is tachyonic with

$$
f_{1}<0 \text {. }
$$

This unfortunately implies the loss of perturbative unitarity due to positivity bounds requiring $f_{1}>0$ [150]. We can now try to simplify relation (4.4.3) using that $\mathcal{H}^{2} a^{-2}=H^{2}$ in de Sitter. In the next step, we make it explicit that the inequality is satisfied by substituting for $M_{w}^{2}=-\left|M_{w}^{2}\right|$. Solving for $M_{c s}$, gives the simple relation

$$
M_{c s}^{2}>\frac{\left|M_{w}\right|^{4}}{4\left(\left|M_{w}\right|^{2}-4 H^{2}\right)} .
$$

As this involves dynamical quantities, in the more general cases, it translates to a constraint on the time-evolution of the theory. But there is a special case, which we would like to emphasize, where the dynamical contributions completely drop out. For specific choices ${ }^{9}$ of the functions $f_{1}$ and $f_{2}$ one finds a simple relationship between the physical scales involved ${ }^{10}$, namely $\Lambda^{2}>H^{2}$. Notice that this relationship is

\footnotetext{
${ }^{9}$ The functions $f_{1}$ and $f_{2}$ have to depend on time in such a way so that all time-variables exactly cancel in (4.4.5). We give such an example later in the text.

${ }^{10}$ For simplicity, here we have neglected a small constant contribution to the leading order term.
} 


\section{CHIRAL PRIMORDIAL GRAVITATIONAL WAVES IN EXTENDED THEORIES OF SCALAR-TENSOR GRAVITY}

always satisfied in EFTs as the scale where we expect to find new physics is always larger than the energy scale of inflation (i.e. in our case we have $\Lambda \simeq M_{P l}>H^{2}$ ). A similar conclusion was reached in [112] in the case of slow-roll inflation where dynamical parameters depend only weakly on time.

\subsection{A toy model}

We proceed to solve the equations of motion in (4.3.19). We choose to work in pure de Sitter $\left(a=-(H \eta)^{-1}\right)$ and look at a particularly simple example, where $^{11}$ $\left|f_{1}\right|=8\left|f_{10}\right|$ and

$$
f_{2}=8 f_{20} \int \eta^{-1} \mathrm{~d} \eta,
$$

with the coupling constants having magnitudes of $\left|f_{10}\right|, f_{20} \sim \mathcal{O}(1) \sim 1$. This way we have that $\bar{f}_{1}=f_{1}$ and $\bar{f}_{2}=f_{2}$. We introduce the relative parameters $\omega_{1}, \omega_{2}, g_{1}$ and $g_{2}$. These are defined as follows:

$$
\omega_{1}=\frac{f_{1}}{c_{T}}, \quad \omega_{2}=f_{1} c_{T}, \quad g_{1}=\frac{f_{2}}{c_{T}^{2}}, \quad g_{2}=f_{2} .
$$

This way the relationships between the functions $\omega_{1}, \omega_{2}, g_{1}$, and $g_{2}$, simplify to

$$
\omega_{1}=\frac{\omega_{2}}{c_{T}^{2}} \quad \text { and } \quad g_{1}=\frac{g_{2}}{c_{T}^{2}}
$$

We can make contact with (4.3.17) by making the following identifications $\gamma=$ $\omega_{1}\left(a M_{P l}\right)^{-2}, \delta=\omega_{2}\left(a M_{P l}\right)^{-2}, \varepsilon=g_{1}^{\prime}\left(a M_{P l}\right)^{-2}$ and $\zeta=g_{2}^{\prime}\left(a M_{P l}\right)^{-2}$. Here we have set $\alpha=\beta=0$ as, with our definitions, these terms only add a negligible constant to the leading order term. The equations of motion simplify to

$$
\left(\mu^{s}\right)^{\prime \prime}+\left[k^{2} \frac{\left(c_{T}^{2}-\frac{k^{2} \omega_{2}}{a^{2} M_{P l}^{2}}-\frac{\lambda^{s} k g_{2}^{\prime}}{a^{2} M_{P l}^{2}}\right)}{\left(1-\frac{k^{2} \omega_{1}}{a^{2} M_{P l}^{2}}-\frac{\lambda^{s} k g_{1}^{\prime}}{a^{2} M_{P l}^{2}}\right)}-\frac{\left(z^{s}\right)^{\prime \prime}}{z^{s}}\right] \mu^{s}=0 .
$$

The speed of gravitons contains corrections coming from the higher-order operators which, as we shall soon see, they drop out. The effective potential is given in terms of

$$
z^{s}=a \sqrt{1-\frac{k^{2} \omega_{1}}{a^{2} M_{P l}^{2}}-\frac{\lambda^{s} k g_{1}^{\prime}}{a^{2} M_{P l}^{2}}} .
$$

\footnotetext{
${ }^{11}$ Here we have reinstated the numerical factors that we had absorbed earlier into the functions $f_{1}$ and $f_{2}$.
} 
To ensure stability for all modes, within the regime of validity of the EFT, we derive the simple constraint

$$
\left(z^{s}\right)^{2}>0 \Rightarrow M_{c s}^{2}>\frac{\left|M_{w}\right|^{2}}{4 c_{T}^{3}}, \quad f_{1}<0
$$

which can be re-expressed as a lower bound to the speed of gravitons

$$
c_{T}^{3}>\frac{\left|M_{w}\right|^{2}}{4 M_{c s}^{2}} \simeq \frac{H^{2}}{M_{P l}^{2}} .
$$

Comparing this to (4.3.11) it is now evident that the Chern Simons instability will kick in for parametrically small values of $c_{T}$, in the limit $E^{2} / \Lambda_{*}^{2} \rightarrow 1$. With all this in place we can now treat the higher-order corrections as being energetically negligible for as long as we remain within the regime of validity of the EFT. From now on we make explicit the minus sign in $f_{1}=-\left|f_{1}\right|<0$, as demanded by (4.5.6) and, for simplicity, drop the absolute notation. The effective potential is expressed as

$$
\begin{aligned}
\frac{\left(z^{s}\right)^{\prime \prime}}{z^{s}} & =\frac{2}{\eta^{2}}-\frac{1}{\eta}\left(\frac{2 \omega_{10} k^{2} \eta-g_{10} \lambda^{s} k}{1+\omega_{10} k^{2} \eta^{2}-g_{10} \lambda^{s} k \eta}\right) \\
& -\frac{1}{4}\left(\frac{2 \omega_{10} k^{2} \eta-g_{10} \lambda^{s} k}{1+\omega_{10} k^{2} \eta^{2}-g_{10} \lambda^{s} k \eta}\right)^{2}+\left(\frac{\omega_{10} k^{2}}{1+\omega_{10} k^{2} \eta^{2}-g_{10} \lambda^{s} k \eta}\right)
\end{aligned}
$$

where we have simplified our arguments by introducing the following redefinitions

$$
\omega_{10}=\frac{8 f_{10} H^{2}}{c_{T} M_{P l}^{2}}, \quad \omega_{20}=\frac{8 f_{10} c_{T} H^{2}}{M_{P l}^{2}}, \quad g_{10}=\frac{8 f_{20} H^{2}}{c_{T}^{2} M_{P l}^{2}}, \quad g_{20}=\frac{8 f_{20} H^{2}}{M_{P l}^{2}} .
$$

Using the definitions given above it is easy to see that the corrections to the leading order contribution to the speed of gravitons drop out, giving

$$
\tilde{c}_{T}^{2}=\frac{c_{T}^{2}+\omega_{20} k^{2} \eta^{2}-g_{20} \lambda^{s} k \eta}{1+\omega_{10} k^{2} \eta^{2}-g_{10} \lambda^{s} k \eta}=c_{T}^{2} .
$$

Note that if we set $\omega_{10}=0$ and $c_{T}=1$ in (4.5.8) we obtain the form of the effective potential in [110]. Similarly, if we set $g_{10}=0$ we correctly recover the equation for inflation which corresponds to a harmonic oscillator with a time-dependent frequency. Taking the small scale limit $(|k \eta| \rightarrow \infty)$ of the equation of motion gives

$$
\left(\mu^{s}\right)^{\prime \prime}+k^{2} c_{T}^{2} \mu^{s}=0 .
$$

This is satisfied by the Bunch-Davies vacuum solution where gravitons propagate with a speed $c_{T}$, given by 


\section{CHIRAL PRIMORDIAL GRAVITATIONAL WAVES IN EXTENDED THEORIES OF SCALAR-TENSOR GRAVITY}

$$
\lim _{|k \eta| \rightarrow \infty} \mu^{s}=-\frac{e^{-i c_{T} k \eta}}{\sqrt{2 c_{T} k}} .
$$

Taking the large scale limit $(|k \eta| \rightarrow 0)$, gives

$$
\left(\mu^{s}\right)^{\prime \prime}+\left(k^{2} c_{T}^{2}-\frac{2}{\eta^{2}}-\frac{g_{10} k \lambda^{s}}{\eta}\right) \mu^{s}=0
$$

where, for simplicity, we have neglected subdominant contributions to the graviton speed. Equation (4.5.13) can be solved exactly. We bring it into the Whittaker form

$$
\left(\mu^{s}\right)^{\prime \prime}+\left[-\frac{1}{4}+\frac{\nu}{\chi}+\frac{\frac{1}{4}-\mu^{2}}{\chi^{2}}\right] \mu^{s}=0,
$$

by making a substitution of the form

$$
\chi=2 i c_{T} k \eta
$$

and therefore, $\mu^{s}=\mu^{s}(\mathbf{k}, \chi)$. Hence, equation to solve is

$$
\left(\mu^{s}\right)^{\prime \prime}+\left[-\frac{1}{4}+\frac{i g_{10} \lambda^{s}}{2 c_{T} \chi}+\frac{(-2)}{\chi^{2}}\right] \mu^{s}=0 .
$$

Solutions are in terms of Whittaker functions ${ }^{12}$ (the details can be found in Appendix A.10). The tensor power spectrum, per polarization, is found to be

$$
k^{3} P_{h}^{s}(k)=\frac{1}{\pi^{2}} \frac{H^{2}}{M_{P l}^{2} c_{T}^{3}}\left|\Gamma\left(2-\frac{i g_{10} \lambda^{s}}{2 c_{T}}\right)\right|^{-2} e^{-\frac{\pi \lambda^{s} g_{10}}{2 c_{T}}},
$$

and it is scale-invariant, as expected. We conclude that, for $\lambda=-1$, the left modes are enhanced.

\subsubsection{Chirality enhancement}

In this work we are interested to parametrically approach the regime at which maximal parity violation occurs. Therefore we consider the non-relativistic limit $c_{T} \ll 1$. To find the maximum possible enhancement for the left modes we have to take into account the condition in (4.3.11) which is equivalent to the lower bound for $c_{T}$ in (4.5.7). We find that as we parametrically approach the limit $E^{2} / \Lambda_{*}^{2} \rightarrow 1$ the exponential argument in (4.5.17), namely

\footnotetext{
12 The prescription for finding inflationary solutions in terms of the Whittaker equations can be found in $[110,113,151]$.
} 


$$
e^{\frac{\pi g_{10}}{2 c_{T}}} \simeq e^{\frac{4 \pi H^{2}}{c_{T}^{3} M_{P l}^{2}}} \simeq e^{\frac{H^{2}}{\Lambda_{*}^{2}}},
$$

becomes of order one ${ }^{13}$ leading to maximal parity violation with circular polarization ratio $\Pi \rightarrow 1$ indicating a fully left-handed signal. This is the point at which we expect the quadratic theory to break down and the Chern Simons instability to appear in our system. If we were to include cubic interactions we would also expect the theory, at this point, to be strongly coupled resulting to large parity violation in tensor non-Gaussianity.

At this point we would like to emphasize that by using this framework it becomes explicit that the Chern Simons instability is a consequence of the strongly coupled theory. From this we conclude that if maximal parity violation is to occur new physics are ought to be included. This translates to adding extra degrees of freedom so that we can admit a weakly coupled description of our system which could enable us to consistently parametrize it when we extrapolate it to higher energies. Such approaches have been attempted in the scalar sector in $[123,124]$ where they studied weakly coupled completions of the EFTI action in [122]. It would be interesting to do something similar for the tensor sector.

Our conclusions are in agreement with the literature, as so far we have not been able to produce an observable amount of circular polarization of gravitational waves in single field inflation. Our approach is to be understood as being complimentary to previously examined cases in the literature.

Another important constraint may come from requiring a small scalar-tensor ratio as per the results from the Planck collaboration [15]. Looking at the tensor power spectrum in (4.5.17) we see there is an inverse factor of $c_{T}^{3}$ scaling the overall power spectrum, therefore, when we enhance chirality at the same time we also enhance the overall amplitude of the gravitational waves, so care must be taken. Taking again the limit $E^{2} / \Lambda_{*}^{2} \rightarrow 1$ and summing over the polarizations we find the power spectrum can take the maximal value ${ }^{14}$

$$
k^{3} P_{h}^{s}(k) \sim \mathcal{O}(1),
$$

leading to a large tensor amplitude. Either way, if the scalar-tensor ratio is too small then it would be very difficult to detect circular polarization due to cosmic variance. Therefore, a large tensor amplitude is preferable. The presence of $c_{T}^{-3}$ in the power spectrum could be used to parametrically enhance the amplitude of gravitational waves which also enhances detectability.

\subsection{Relation between frames}

It has been shown that disformal transformations cannot remove four- or higherderivative corrections to the quadratic action for tensors [152]. Additionally, suffi-

\footnotetext{
${ }^{13}$ Here we used relation (4.3.11) to substitute for $c_{T}^{-3} \sim M_{P l}^{2} / H^{2}$.

14 For simplicity, here we have ignored various order one parameters.
} 


\section{CHIRAL PRIMORDIAL GRAVITATIONAL WAVES IN EXTENDED THEORIES OF SCALAR-TENSOR GRAVITY}

ciently complicated theories do not guarantee to have an Einstein frame. For example, consider the scalar-tensor action in (4.3.17) for arbitrary parameters $\alpha-\zeta$. Upon disformal transformation, in the new frame, one may expect to find a quadratic action in terms of canonical Einstein gravity (i.e. as in [135]) but with a non-standard higher-curvature extension. As here we work with theories that go beyond Einstein's gravity, we do not technically consider physics in the Einstein frame. Therefore, in what follows we simply dub different frames as A and B.

In our case, we started in a frame, say A, where the action in (4.3.1) is described by the canonical Einstein quadratic term plus the extension to gravity given by Weinberg in [80]. This theory is understood to be valid up to some fixed heavy mass scale $\Lambda \simeq M_{P l}$. We then moved our formulations to another frame, say $\mathrm{B}$, by disformally mapping the operators at the level of the perturbations. We found an action (4.3.6) in which gravitons propagate with a non-trivial speed $c_{T}$. We demonstrated that if $c_{T}$ becomes parametrically small for a fixed scale $\Lambda \simeq M_{P l}$ it could spoil the validity of the EFT. We showed that this is the point where we expect maximal parity violation to occur resulting to the presence of the Chern Simons instability. Additionally cubic operators would become important resulting into tensor modes appearing strongly coupled well below the energy scale $\Lambda \simeq M_{P l}$.

It is to be understood that we work, essentially, with a different representation of extensions to Einstein's gravity. In this sense, the A and B frame theories are said to be mathematically equivalent [153]. In the limit where $c_{T}=1$ the theories are also said to be physically equivalent. On the other hand, when we consider arguments on the physical equivalence between frames, at the non-relativistic limit $c_{T} \ll 1$, we stumble across a noticeable difference. We cannot bypass the effective field theory machinery which instructs us that we should have to include additional degrees of freedom to the B frame theory, so that we can produce a theory that is valid and consequently weakly coupled all the way from the energy scale of inflation $H$ to the fixed cutoff scale $\Lambda \simeq M_{P l}$. This is very likely to affect the effective description of the theory (as in $[123,124]$ ) and therefore, the observables as they are sensitive to the higher-curvature corrections. We find that arguments on physical equivalence between frames are more difficult to reconcile once we go beyond leading order effects.

Finally, let us remark that one could build an effective description having the same form with (4.3.6) by starting with operators that do not admit an inverse disformal transformation. These were examined in [154].

\subsection{Outlook}

In this work we re-examined the problem of parity violation in single field inflation. In particular, we looked for a systematic way to parametrically approach the scale at which maximal parity violation occurs. We achieved this by introducing a small parameter, by means of a disformal transformation, whose effect was to suppress the heavy cutoff scale $\Lambda \simeq M_{P l}$ of the effective theory leading to parametrically large chiral tensor fluctuations.

We found that sub-leading quadratic operators can become important signalling 
the presence of the Chern Simons instability. This inevitably implies the existence of non-trivial cubic interactions which could stand the theory strongly coupled for parametrically small values of the parameter $c_{T}$ and, consequently, lead to large parity violation in tensor non-Gaussianity. We showed that by using this framework it becomes explicit that the Chern Simons instability is a consequence of the strongly coupled theory.

We concluded that, at this point, a change in the physical description of the theory is necessary so that one can consistently parametrize our system as the energy increases up to the heavy cutoff scale $\Lambda \simeq M_{P l}$. The addition of new degrees of freedom could help, in a consistent manner, to maintain sufficient parity violation while at the same time ensure stability of the modes. We hope to examine this in a future work.

Our approach is to be understood as being complimentary to previously examined cases in the literature. In particular, extra field content could be incorporated into our EFT framework in a systematic way.

At this point we would like to emphasize that our work is only indicative of the many open questions one needs to carefully tackle when considering higher-order extensions to Scalar-Tensor gravity, especially in the presence of parity violation. We do not wish to answer these questions in one go. As a first attempt we kept the formulations as simple as possible and only tentatively looked at these problems. Indeed, more complicated effective descriptions can come from considering disformal transformations of operators at the covariant level. Below we address the possible directions one can take so that progress can be made in the future.

The next step would be to find a phenomenologically viable inflationary model and do a complete and concrete analysis in quasi-de Sitter. Additionally, one could take into account the complete set of non-redundant six-derivative operators, as higher-curvature terms can become important. Such considerations are subject to finding a field redefinition that can ensure second-order equations of motion. It would also be interesting to see how the bispectrum is affected in this setup by considering cubic operators. In particular we are interested in the regime in which these contributions become important, leading to large tensor non-Gaussianity.

Finally, at the end of inflation the scalar field has reached its minimum $(\phi=$ const.) and the gravitational Chern Simons term becomes a total derivative. Therefore, we do not expect to find parity violation in the subsequent evolution of the classical gravitational dynamics [112]. On the other hand, the six-derivative parity violating terms may not necessarily become surface terms which may affect postinflationary predictions. This deserves more investigation. 


\section{Non-attractor inflation}

\subsection{Abstract}

We discuss a new mechanism to enhance the spectrum of primordial tensor fluctuations in single field inflationary scenarios. The enhancement relies on a transitory non-attractor inflationary phase, which amplifies the would-be decaying tensor mode and gives rise to a growth of tensor fluctuations at superhorizon scales. We show that the enhancement produced during this phase can be neatly treated via a tensor duality between an attractor and non-attractor phase, which we introduce. We illustrate the mechanism and duality in a kinetically driven scenario of inflation with non-minimal couplings between the scalar and the metric.

\subsection{Introduction}

The linearized action for the comoving scalar curvature fluctuation $\mathcal{R}$, in standard single-field inflation, is given by $[38,155]$

$$
S^{(0)}=\int \mathrm{d} \tau \mathrm{d}^{3} x \frac{z_{S}^{2}}{2}\left[\left(\mathcal{R}^{\prime}\right)^{2}-(\nabla \mathcal{R})^{2}\right], \quad z_{S} \equiv a \frac{\dot{\phi}}{H}
$$

where $\phi$ is a homogeneous scalar field and prime denotes differentiation with respect to the conformal time $\tau$, while dots are reserved for differentiation with respect to the cosmic time $t$. We can obtain the evolution equations in Fourier space, which are expressed in terms of the canonically normalized variable $u=z_{S} \mathcal{R}$ and are given by

$$
u^{\prime \prime}+\left(k^{2}-\frac{z_{S}^{\prime \prime}}{z_{S}}\right) u=0,
$$

where $v=v(\mathbf{k}, \tau)$. This is nothing more than the equation of a harmonic oscillator with a time-dependent mass $m^{2}(\tau)=-z_{S}^{\prime \prime} / z_{S}$. The perturbations are quantized using equal-time commutation relations. At early times, when the perturbations are well within the horizon $(k \gg a H)$, we simply get a Klein-Gordon equation

$$
u^{\prime \prime}+k^{2} u=0
$$


This correctly defines the Bunch-Davies vacuum solution

$$
\lim _{|k \eta| \rightarrow \infty} u=\frac{1}{\sqrt{2 k}} e^{-i k \eta}
$$

On the other hand, at superhorizon scales $(k \ll a H)$, we find the general solution

$$
\lim _{k \eta \rightarrow 0} u=c_{1}+c_{2} \int^{y} \frac{\mathrm{d} y^{\prime}}{z_{S}^{2}\left(y^{\prime}\right)}
$$

where we have used the substitution $y=k \tau$. In the standard case of slow-roll inflation, the effective potential is defined to be

$$
z_{S}=\frac{a \dot{\phi}}{H}
$$

At large scales where $\dot{\phi}$ and the Hubble parameter $H$ are taken to be constant, we have that $z_{S} \sim a$ is an increasing function of time and we say that inflation is in an attractor phase. From this it follows that the first term in (5.2.5) is growing, while the second is decaying. As a consequence $\mathcal{R} \simeq$ const is conserved at large scales, resulting in a scale invariant spectrum [79].

However, when we study deviations from the canonical inflation paradigm, there are cases where, for a short period, $z$ can be a rapidly decreasing function of time. Then the inflationary evolution is no longer an attractor. In that case the second term in (5.2.5) becomes a growing mode. This in turn enhances the amplitudes of modes, leaving the horizon during that time, leading to new observational signatures in the power spectrum. This can occur for example in models where the scalar derivative rapidly decreases for a short period, as in inflection point ultra slow-roll and in constant roll inflationary systems (see e.g. [156-163]) or in the Starobinsky model due to a rapid change in the potential slope [164]. Recently these scenarios have received a renewed interest, since an amplification of scalar perturbations can lead to the production of primordial black holes from single field inflation (see e.g. [165-167] for general reviews and [168-177] for specific models).

Although the non-attractor phase of inflation lies well outside the slow-roll regime, a duality exists [178] which allows an analytical description of the statistical features of the enhanced spectrum of fluctuations.

The aim of this work is to present a framework that can produce a similar enhancement of primordial tensor modes during a phase of non-attractor singlefield inflation. This is phenomenologically interesting because interferometers can probe a stochastic background of gravitational waves at much smaller scales (see the textbooks $[179,180])$ as opposed to CMB polarization that can be used to probe the amplitude of the gravitational wave background at large CMB scales (see e.g. the reviews $[181,182]$ ). Inflationary models that enhance the primordial tensor spectrum at interferometer scales could be tested with current or future ground or space based interferometers. 
One way to produce such an enhancement is by exploiting instabilities of additional source fields during inflation. For example, primordial gravity waves can be enhanced by coupling fields driving inflation with additional scalars [183-188], U(1) gauge vectors [114,189-192], non-Abelian vector fields [193-203] or Standard Model fields [204]. Another approach is to implement space-time symmetry breaking during inflation. Ways to do so are scenarios of (super)solid inflation (see e.g. [205-214]) or massive gravity/bigravity models, [215-217]. See e.g. [218] for a more extensive survey of various models proposed so far, focussing on the detectability of inflationary tensor modes with LISA.

The aim of this work is to present a mechanism that can enhance the spectrum of primordial tensor fluctuations, in single-field inflation, at arbitrary scales. We do so by considering a model of kinetically driven single field inflation. At superhorizon scales the would-be decaying mode experiences growth for a short period leading to an enhanced primordial tensor spectrum. This is achieved by introducing discontinuities in the kinetic functions which is analogous to systems with discontinuities in the potential, such as in the Starobinsky model [164].

The paper is organized as follows. In Section 5.3 we study the second-order action for primordial tensor fluctuations in single-field inflation. We identify conditions for obtaining a large enhancement in the tensor spectrum. This is achieved by realizing a non-attractor phase for tensors which only lasts for a few $N$-folds. During that time the would-be decaying tensor mode is enhanced. These conditions are analogous to the requirements discussed in various works, starting with [219-221], for enhancing scalar modes during non-attractor phases and motivate our search for models of inflation with specific non-minimal couplings of tensors to the inflationary scalar field.

In order to gain analytic control of the dynamics of fluctuations in a non-attractor phase, we identify a criterium, in Section 5.4, which we call tensor duality, that ensures identical behaviour, up to an overall factor, for the dynamics of perturbations in two different regimes of the inflationary evolution. This is the generalization to the tensor case of the duality discussed by Wands [178] for the scalar sector. We determine the tensor dual of a phase of standard slow-roll inflation, which corresponds to a period of non-attractor inflation, resulting to a scale invariant spectrum of tensor fluctuations which is amplified with respect to the standard case.

Using this tensor duality as a guide, in Section 5.5 we build and analyse in detail a representative model of single-field kinetically driven inflation, belonging to the G-inflation set-up of [36], which is able to amplify tensor modes during the non-attractor era. Our system is analogous to the Starobinsky model [164], where instead of having discontinuities in the potential, we have a discontinuity in the kinetic functions which causes a short non-attractor phase. Through the tensor duality, we are then able to analytically investigate the dynamics of fluctuations during the non-attractor era, showing that the amplitude of the spectra of tensor (and scalar) fluctuations increases by several orders of magnitude with respect to a standard slow-roll regime.

Finally, we conclude in Section 5.8 with a discussion of possible future directions to explore and provide technical Appendices for some of the results in the main text. 


\subsection{Formulation}

The action for G-inflation for tensor perturbations [36], is given by $\left(M_{P l}=1\right)$

$$
S^{(2)}=\frac{1}{8} \int \mathrm{d} t \mathrm{~d}^{3} x a^{3}\left[\mathcal{G}_{T} \dot{h}_{i j}^{2}-\frac{\mathcal{F}_{T}}{a^{2}}\left(\nabla h_{i j}\right)^{2}\right],
$$

where $\mathcal{G}_{T}$ and $\mathcal{F}_{T}$ are defined as

$$
\begin{aligned}
& \mathcal{F}_{T}=2\left[G_{4}-X\left(\ddot{\phi} G_{5 X}+G_{5 \phi}\right)\right], \\
& \mathcal{G}_{T}=2\left[G_{4}-2 X G_{4 X}-X\left(H \dot{\phi} G_{5 X}-G_{5 \phi}\right)\right],
\end{aligned}
$$

and $h_{i j}$ is the transverse traceless spin-2 tensor perturbation. Going to conformal time, with

$$
\frac{\mathrm{d} t}{a}=\mathrm{d} \tau
$$

this becomes

$$
S_{T}^{(2)}=\frac{1}{8} \int \mathrm{d} \tau \mathrm{d}^{3} x a^{2}\left[\mathcal{G}_{T}\left(\partial_{\tau} h_{i j}\right)^{2}-\mathcal{F}_{T}\left(\nabla h_{i j}\right)^{2}\right]
$$

where the time-dependent factors $\mathcal{G}_{T}$ and $\mathcal{F}_{T}$ scale the spatial and time parts of the quadratic action. The speed of gravitons is given by

$$
c_{T}^{2}=\frac{\mathcal{F}_{T}}{\mathcal{G}_{T}} .
$$

It is convenient to define a new time variable

$$
\mathrm{d} y=\frac{c_{T}}{a} \mathrm{~d} t=c_{T} \mathrm{~d} \tau .
$$

With this redefinition the action for tensor fluctuations describes a free field propagating in a time-dependent background, given by

$$
S_{T}^{(2)}=\frac{1}{2} \int \mathrm{d} y \mathrm{~d}^{3} x z_{T}^{2}(y)\left[\left(\partial_{y} h_{i j}\right)^{2}-\left(\nabla h_{i j}\right)^{2}\right] .
$$

In terms of the above, the effective potential for tensors is given by

$$
z_{T}^{2}=\frac{a^{2}}{4} c_{T} \mathcal{G}_{T}=\frac{a^{2}}{4} \sqrt{\mathcal{F}_{T} \mathcal{G}_{T}}
$$


The evolution equations for tensors in Fourier space read

$$
h_{i j}^{\prime \prime}+2 \frac{z_{T}^{\prime}}{z_{T}} h_{i j}^{\prime}+k^{2} h_{i j}=0
$$

At superhorizon scales $\left(k^{2} \ll\left|z_{T}^{\prime \prime} / z_{T}\right|\right)$ the last term in (5.3.9) can be neglected, giving the general solution

$$
h_{i j}(y)=\mathcal{C}_{1}+\mathcal{C}_{2} \int^{y} \frac{\mathrm{d} y^{\prime}}{z_{T}^{2}\left(y^{\prime}\right)},
$$

with $\mathcal{C}_{1}, \mathcal{C}_{2}$ being integration constants. In the standard case of slow-roll inflation $z_{T}$ increases rapidly with time so that the second term becomes negligible. The usual prescription is to neglect this term and fix the constant $\mathcal{C}_{1}$ by matching the suband superhorizon solutions at horizon crossing. This means that tensor modes are conserved at superhorizon scales.

In what follows, we consider the case where, for a short period, $z_{T}$ is a decreasing function of time, i.e. the second term in (5.3.9) changes sign so that the second term in (5.3.10) starts to grow and dominates the tensor dynamics. Consequently the tensor modes exiting the horizon at that time are enhanced as they do not "freeze" like in the usual case of slow-roll inflation, but instead they continue to grow at superhorizon scales. Since the would-be decaying mode is no longer suppressed by inverse powers of the scale factor, the system enters a non-attractor regime for the tensor sector:

$$
\frac{z_{T}^{\prime}}{z_{T}}<0 \Rightarrow \text { non-attractor phase } \Rightarrow \text { amplification of tensor modes. }
$$

A short period where the system exhibits such behaviour can be realized if the functions $\mathcal{F}_{T}$ and $\mathcal{G}_{T}$ in (5.3.8) are heavily time-dependent [220]. This is similar to mechanisms employed for producing primordial black holes from single field inflation [221].

We propose that this mechanism can be used to amplify the tensor spectrum resulting from inflation, as long as there is a short period where slow-roll inflation is violated. This introduces discontinuities in the field dynamics making it difficult to treat the problem analytically as we can no longer analytically describe it by means of the usual slow-roll formulae. For this reason we employ a tensor duality which enables us to have analytic control during the phase that the system experiences heavy time-dependence. Next, we briefly review this tensor duality.

\subsection{Tensor duality}

The idea of duality for scalar fluctuations was first explored in [178], followed by the works in [222-229]. It allows one to identify scenarios that are able to produce a 
scale invariant spectrum of fluctuations without invoking a phase of quasi-de Sitter expansion, as in bouncing cosmologies (see e.g. [230,231] for recent reviews on this broad topic). The same concept can be applied to the description of brief transient phases of non-attractor evolution during inflation, as first discussed in [220,221], explaining some of the key features of scalar power spectra in these regimes. In this Section we develop further this idea, extending it to the physics of tensor modes, with the aim of setting the stage for determining scenarios that enhance tensor fluctuations during non-attractor inflationary regimes.

We start by looking at the action in (5.3.7) which can be rewritten in terms of the canonically normalized tensor field

$$
h_{i j}=\frac{q_{i j}}{z_{T}} .
$$

In this way, we bring the quadratic action for tensors to the standard form, given by

$$
S_{T}^{(2)}=\frac{1}{2} \int \mathrm{d} y \mathrm{~d}^{3} x\left[\left(q_{i j}^{\prime}\right)^{2}-\left(\nabla q_{i j}\right)^{2}+\frac{z_{T}^{\prime \prime}}{z_{T}} q_{i j}^{2}\right]
$$

where we use a prime to denote derivatives with respect to the variable $y$. This corresponds to the action of a harmonic oscillator with a time-dependent effective mass $\sim-z_{T}^{\prime \prime} / z_{T}$. In [178] it was shown that $z_{T}^{\prime \prime} / z_{T}$ remains invariant under transformations of the effective potential $z_{T}(y)$, which has the general form

$$
\tilde{z}_{T}(y)=z_{T}(y)\left(c_{1}+c_{2} \int^{y} \frac{\mathrm{d} y^{\prime}}{z_{T}^{2}(y)}\right)
$$

where $c_{1}, c_{2}$ are constants. This process leaves the quadratic action and therefore the equations of motion unchanged. Consequently the solutions to the evolution equations $q_{i j}$ remain invariant, i.e. under this transformation, we have that

$$
\frac{\tilde{z}_{T}^{\prime \prime}}{\tilde{z}_{T}}=\frac{z_{T}^{\prime \prime}}{z_{T}}
$$

which leaves the canonically normalized tensor field $q_{i j}$ unaffected. Since the action in (5.4.2) contains the same canonical tensor field $q_{i j}$ after the redefinition of $z_{T}$, we can use this to relate the original tensor fluctuations $h_{i j}$ in (5.4.1) to the new tensor fluctuations $\tilde{h}_{i j}=q_{i j} / \tilde{z}_{T}$, by

$$
\left\{\begin{array}{l}
h_{i j}=q_{i j} / z_{T} \\
\tilde{h}_{i j}=q_{i j} / \tilde{z}_{T}
\end{array} \quad \Rightarrow \tilde{h}_{i j}=\left(\frac{z_{T}}{\tilde{z}_{T}}\right) h_{i j},\right.
$$

where we call $\tilde{h}_{i j}$ the tensor dual of $h_{i j}$. The quadratic action describing the dynamics of $\tilde{h}_{i j}$ has the same structure as (5.3.7), but contains $\tilde{z}_{T}$ instead of $z_{T}$. From this 
it follows that the power spectra associated with these two tensor fluctuations are simply related by

$$
P_{\tilde{h}}=\left(\frac{z_{T}}{\tilde{z}_{T}}\right)^{2} P_{h}
$$

This implies that if we have analytic control over the dynamics of the perturbations $h_{i j}$ and their spectrum, we can easily control the spectrum for the dual fluctuations $\tilde{h}_{i j}$. From (5.4.6) we see that if the ratio $z_{T} / \tilde{z}_{T}$ is large, the dual tensor spectrum is enhanced.

\subsubsection{The tensor dual of a slow-roll phase}

We can use this duality to make contact between the dynamics of the tensor fluctuations $h_{i j}$ and their dual $\tilde{h}_{i j}$. The dynamics of the tensor field $h_{i j}$, in the slow-roll regime, were analysed in [36] where they considered a quasi-de Sitter background with $\mathcal{F}_{T}$ and $\mathcal{G}_{T}$ taken to be almost constant. The powerspectrum was found to be almost scale invariant, with amplitude at horizon exit

$$
P_{h}=\frac{2 H^{2}}{\pi^{2}} \frac{\mathcal{G}^{\frac{1}{2}}}{\mathcal{F}^{\frac{3}{2}}}
$$

where here we neglect the (weak) time-dependence of the Hubble parameter and of the functions $\mathcal{F}_{T}, \mathcal{G}_{T}$ (see e.g. [36] for more complete expressions). In the quasi-de Sitter slow-roll phase, the effective potential in (5.3.8), is given by

$$
z_{T}^{2}=\frac{a^{2}}{4} \sqrt{\mathcal{F}_{T} \mathcal{G}_{T}} \sim \text { const. } \times a^{2}
$$

We are interested in the case where the dual tensor spectrum $P_{\tilde{h}}$ in (5.4.6) is enhanced, i.e. that's when $z_{T} / \tilde{z}_{T} \gg 1$. We can use our knowledge of $z_{T}$ during the slow-roll phase, i.e. $z_{T}^{2} \sim a^{2}$ in (5.4.8), to make contact with $\tilde{z}_{T}$ where, for simplicity, we work in a pure de Sitter background and take $\mathcal{F}_{T}$ and $\mathcal{G}_{T}$ to be constant. Using relation (5.4.3), we find

$$
\partial_{y}\left(\frac{\tilde{z}_{T}}{z_{T}}\right)=\partial_{y}\left(c_{1}+c_{2} \int^{y} \frac{\mathrm{d} y^{\prime}}{z_{T}^{2}(y)}\right) \propto \frac{1}{z_{T}^{2}} .
$$

We can express this, using (5.3.6) in terms of the old time coordinate $t$, as

$$
\partial_{t}\left(\frac{\tilde{z}_{T}}{z_{T}}\right) \propto \frac{1}{a^{3}}
$$

where $a \sim e^{H t}$ in de Sitter. Integrating this result gives 


$$
\frac{\tilde{z}_{T}}{z_{T}} \propto \frac{1}{a^{3}}
$$

from which we get

$$
\tilde{z}_{T}^{2} \propto \frac{1}{a^{4}}
$$

where we have used that during the slow-roll phase $z_{T}^{2} \sim a^{2}$. From this we see that in the slow-roll phase $z_{T}$ increases with time, while in the dual tensor phase $\tilde{z}_{T}$ decreases, meaning that we are in a non-attractor regime in which tensor fluctuations can grow at superhorizon scales, as we discussed in Section 5.3. From this we find that the power spectrum of the dual tensor perturbations, given in (5.4.6), is almost scale invariant and considerably enhanced

$$
P_{\tilde{h}}=\left(\frac{z_{T}}{\tilde{z}_{T}}\right)^{2} P_{h} \propto a^{6} P_{h} .
$$

Since the scale factor $a \sim e^{H t}$ is an increasing function of time, this mechanism allows one to considerably amplify the tensor spectrum in the regime where the relations (5.4.9), (5.4.10), (5.4.11) and (5.4.12)hold. This mechanism is the analogue for the tensor sector, of the mechanism introduced by [178] and well explored in the scalar sector by $[175]$.

As we have taken that during slow-roll $\sqrt{\mathcal{F}_{T} \mathcal{G}_{T}} \simeq$ const., then using the result in (5.4.12), we find that

$$
\tilde{z}_{T}^{2}(y)=\frac{a^{2}}{4} \sqrt{\tilde{\mathcal{F}}_{T} \tilde{\mathcal{G}}_{T}} \propto \frac{1}{a^{4}},
$$

which implies that during the dual tensor phase we must have that

$$
\sqrt{\tilde{\mathcal{F}}_{T} \tilde{\mathcal{G}}_{T}} \propto \frac{1}{a^{6}} .
$$

This defines a non-attractor regime for tensor fluctuations. In the next Section we present an explicit example in which we realise such regime during a short phase of non-attractor inflationary evolution.

\subsection{Amplifying tensor modes and realising the tensor duality}

\subsubsection{Our aims}

We now seek a realization of the amplification mechanism and the tensor duality of Sections 5.3 and 5.4 in a single-field inflationary system, whose background evolution 
is controlled by the scalar field $\phi$. When non-minimal derivative couplings between scalar and metric are present, the functions $\mathcal{F}_{T}$ are $\mathcal{G}_{T}$ can have non-trivial timedependent profiles. We look for a set-up where they can be expressed as

$$
\mathcal{G}_{T} \propto \frac{\dot{\phi}^{2}}{H^{2}} \quad \text { and } \quad \mathcal{F}_{T} \propto \frac{\dot{\phi}^{2}}{H^{2}}
$$

which implies that

$$
\sqrt{\mathcal{G}_{T} \mathcal{F}_{T}} \propto \frac{\dot{\phi}^{2}}{H^{2}} \quad \text { and } \quad z_{T} \sim a \frac{\dot{\phi}}{H}
$$

The reason for this choice is to 'mimic' the behaviour of the action for scalar fluctuations, as we now explain. There have been several recent studies utilizing the mechanism described in the previous sections to enhance scalar fluctuations (see e.g. [168-175] for recent studies). In particular one can start with the action in (5.2.1) and apply the scalar duality in [178], namely

$$
\tilde{z}_{S}(\tau) \propto z_{S}(\tau) \int^{\tau} \frac{\mathrm{d} \tau^{\prime}}{z_{S}^{2}\left(\tau^{\prime}\right)},
$$

which states that the statistics of scalar fluctuations do not change in regimes related by condition (5.5.3). In the dual of a slow-roll, quasi-de Sitter phase of expansion with $\dot{\phi}=$ const., the scalar velocity must decrease as

$$
\dot{\tilde{\phi}} \propto \dot{\phi} \int \frac{\mathrm{d} t^{\prime}}{a^{3}\left(t^{\prime}\right) \dot{\phi}\left(t^{\prime}\right)} \propto \frac{1}{a^{3}}
$$

precisely the behaviour one encounters in a non-attractor, ultra slow-roll regime of inflation. In the scalar dual of a slow-roll phase, scalar fluctuations are enhanced by a factor $P_{\tilde{\mathcal{R}}} \propto a^{6} P_{\mathcal{R}}$.

Here we extend these ideas to the tensor sector. In particular, we are interested in scenarios in which a phase of de Sitter expansion (where $H$ and $\dot{\phi}$ are approximately constant) is briefly interrupted by a phase of non-attractor inflation with de Sitter expansion, but where $\dot{\phi} \sim 1 / a^{3}$. In this case, one passes from $\sqrt{\mathcal{F}_{T} \mathcal{G}_{T}}=$ const. during slow-roll to $\sqrt{\mathcal{F}_{T} \mathcal{G}_{T}} \propto 1 / a^{6}$ during non-attractor inflation, precisely what we need to amplify the tensor modes and realise the tensor duality (see equations (5.4.15), (5.5.1) and (5.5.2)). The simplest possibility for having a regime where $\dot{\phi}$ transiently decreases during inflation is the scenario of Starobinsky [164] (see Appendix A of [175] for a detailed analysis of this scenario), in which a linear inflationary potential $V(\phi)$ is continuous but has an abrupt change in its slope for a certain value of the scalar field. In this case, the scalar field velocity $\dot{\phi}$ rapidly changes during a short fraction of the inflationary period to adapt its value from the first to the second slow-roll regime which is characterised by a different potential. 
During the transition its value decreases as $\dot{\phi} \sim 1 / a^{3}$ for an appropriate choice of the parameters involved. Whilst the Starobinsky model leads to an enhancement of scalar fluctuations, tensor modes remain small as $\mathcal{F}_{T} \sim \mathcal{G}_{T} \sim 1$.

In what follows we put forward a mechanism that can realize a brief period during which the effective potential is decreasing. We build a version of the Starobinsky model [164], working in the framework of the Horndeski theory introduced earlier in the text. We consider an inflationary system which is kinetically driven by a single scalar field $\phi$ and where the functions $\mathcal{F}_{T}$ and $\mathcal{G}_{T}$ exhibit heavy time-dependence. We select the parameters of the system in such a way such as to ensure that conditions (5.5.1) and (5.5.2) are satisfied, so that during the short non-attractor regime, in which $\dot{\phi} \sim 1 / a^{3}$, tensor modes are enhanced and the tensor duality applies.

Our purpose is specifically to show that our mechanism for enhancing tensor fluctuations can be realised in a toy model for inflation. Therefore, we do not aim at building a realistic inflationary model which matches with CMB observations at large scales and has a realistic exit from inflation. A more realistic set-up will be explored elsewhere.

\subsubsection{The model}

The Horndeski theory was introduced in Section (3.2) where we showed that the Horndeski action is characterized by non-minimal derivative couplings of the scalar field to the metric and where $G_{a}(\phi, X), a=1, \cdots 5$ are generic functions of the scalar field $\phi$ and $X=1 / 2 \partial_{\mu} \phi \partial^{\mu} \phi$. Scenarios of ultra slow-roll, non-attractor G-inflation have been discussed in [232], concentrating on the dynamics of scalar fluctuations. Other single field models of kinetically driven non-attractor inflation have been explored in $[233,234]$ (see also [235]), focussing especially on the enhancement of scalar non-Gaussianity in the squeezed limit.

We are interested in the particular case where a de Sitter inflationary expansion with $H \sim$ const. and $\dot{\phi} \sim$ const. is briefly interrupted by a change to the velocity of the scalar field, namely $\dot{\phi} \sim a^{-3}$. For simplicity, we focus on scenarios of kinetically driven inflation $G_{a}=G_{a}(X)$ and build a version of the Starobinski model [164] in this context, by choosing the following structure for the functions $G_{a}(X)$ :

$$
\begin{aligned}
G_{2}^{(i)} & =\rho_{i} X+\frac{\sqrt{2}}{3} H_{0}^{2} \alpha_{i} \sqrt{X}-V_{i}, \\
G_{3}^{(i)} & =\frac{\sqrt{2}}{3 H_{0}} \delta_{i} \sqrt{X}, \\
G_{4}^{(i)} & =-\frac{\beta_{i}}{6 H_{0}^{2}} X, \\
G_{5}^{(i)} & =\frac{\sigma_{i}}{\sqrt{2} H_{0}^{3}} \sqrt{X},
\end{aligned}
$$

where $H_{0}$ has units of mass and we denote the dual and non-dual phases of inflation with $i=1,2$, respectively. We introduce a discontinuity in the functions $G_{a}$ by demanding that the magnitude of the dimensionless parameters $\alpha_{i}, \beta_{i}, \delta_{i}, \rho_{i}, \sigma_{i}, V_{i}$ 
changes at some time $t=t_{0}$, where $i=1$ corresponds to $t<t_{0}$ and $i=2$ corresponds to $t>t_{0}$. Schematically, we have

$$
\left(\alpha_{i}, \beta_{i}, \delta_{i}, \rho_{i}, \sigma_{i}, V_{i}\right)= \begin{cases}\alpha_{1}, \beta_{1}, \delta_{1}, \rho_{1}, \sigma_{1}, V_{1}, & t<t_{0} \\ \alpha_{2}, \beta_{2}, \delta_{2}, \rho_{2}, \sigma_{2}, V_{2}, & t>t_{0}\end{cases}
$$

With these in place, the slope of the scalar field velocity will abruptly change during inflation (i.e. at time $t_{0}$ ) signalling the start of the dual phase. Abrupt changes/discontinuities at time $t_{0}$ could be smoothed out by introducing step functions, as in [175]. Another important point to make is that our system enters the dual phase, where tensor fluctuations are amplified, only for a short period of time. It is important that the system transitions from kinetic domination to slow-roll inflation, with $z \sim a$, before inflation ends. A graceful exit from inflation could be ensured by allowing $\phi$ to be time-dependent so that we can ensure a standard Einstein-Hilbert term with $G_{4}=$ const. after inflation. We leave these considerations for future investigations.

\subsubsection{Background evolution}

Using the definitions for the functions $G_{a}(X)$ in (5.5.5), the background equations for the scalar field in (3.2.9) read

$$
\frac{\mathrm{d}}{\mathrm{d} t}\left\{a^{3}(t)\left[\alpha_{i} \frac{H_{0}^{2}}{3}+\left(-\rho_{i}+\delta_{i} \frac{H(t)}{H_{0}}+\beta_{i} \frac{H^{2}(t)}{H_{0}^{2}}+\sigma_{i} \frac{H^{3}(t)}{H_{0}^{3}}\right) \dot{\phi}\right]\right\}=0,
$$

where $J$ is given by the expression inside the square brackets and $i=1,2$ correspond to the two phases of evolution, before and after the transition at $t=t_{0}$. We focus on solutions where the scalar field velocity is monotonic (with convention $\dot{\phi}<0$ ) and the scale factor is exponentially increasing (de Sitter space) with constant Hubble parameter $H_{0}$ during the entire inflationary evolution. We first consider the case $t \leq t_{0}$, which corresponds to parameters with $(i=1)$. We solve for $\dot{\phi}$ in (3.2.8), with $J=0$, to find

$$
\dot{\phi}=-\frac{H_{0}^{2} \alpha_{1}}{3\left(-\rho_{1}+\delta_{1}+\beta_{1}+\sigma_{1}\right)}, \quad t \leq t_{0},
$$

where $H(t)=H_{0}$ is the Hubble constant and the scale factor in de Sitter is given by

$$
a(t)=e^{H_{0}\left(t-t_{0}\right)}
$$

Therefore, in the first part of the evolution $\left(t \leq t_{0}\right)$ the scalar field velocity is constant.

Next we consider the case $t \geq t_{0}$, which corresponds to parameters with $(i=2)$. We solve for $\dot{\phi}$ in (3.2.8), with $\bar{J}=c_{2} / a^{3}(t)$, to find 


$$
\dot{\phi}=-\frac{3 c_{2}+H_{0}^{2} \alpha_{2} a^{3}(t)}{3\left(\beta_{2}+\delta_{2}-\rho_{2}+\sigma_{2}\right) a^{3}(t)} .
$$

We ensure that the scalar velocity is continuous by matching the solutions in (5.5.7) and (5.5.9) at time $t=t_{0}$ where $a\left(t_{0}\right)=1$. This is possible if

$$
c_{2}=\frac{H_{0}^{2}}{3}\left(-\alpha_{2}+\frac{\alpha_{1}\left(\beta_{2}+\delta_{2}-\rho_{2}+\sigma_{2}\right)}{\beta_{1}+\delta_{1}-\rho_{1}+\sigma_{1}}\right) .
$$

Substituting for $c_{2}$ into (5.5.9), we find that the scalar velocity for $t \geq t_{0}$ becomes

$\dot{\phi}=-\frac{H_{0}^{2} \alpha_{1}}{3\left(-\rho_{2}+\delta_{2}+\beta_{2}+\sigma_{2}\right)}\left[\alpha_{2}\left(1-\frac{1}{a(t)^{3}}\right)+\frac{\alpha_{1}\left(-\rho_{2}+\delta_{2}+\beta_{2}+\sigma_{2}\right)}{a(t)^{3}\left(-\rho_{1}+\delta_{1}+\beta_{1}+\sigma_{1}\right)}\right], \quad t \leq t_{0}$.

Therefore, in the second part of the evolution $\left(t \geq t_{0}\right)$ the scalar field velocity is allowed to vary. Assuming that for $t \geq t_{0}$ we have $\alpha_{2} \ll \alpha_{1}$ (here we take $\alpha_{2}$ to be very small i.e. $\alpha_{2} \ll 1$ and neglect its contributions all together), the system enters a short phase, lasting from $t=t_{0}$ until $t=t_{1}$, during which the scalar velocity is rapidly decreasing with

$$
\dot{\phi} \simeq-\frac{H_{0}^{2} \alpha_{1}}{3\left(-\rho_{1}+\delta_{1}+\beta_{1}+\sigma_{1}\right) a(t)^{3}} \Rightarrow \dot{\phi} \propto \frac{1}{a(t)^{3}} .
$$

Therefore, the solutions for the scalar equation in (5.5.7) and (5.5.11) produce the desired behaviour for the scalar field velocity.

The scalar field velocity returns to a constant value, at time $t>t_{1}$, when the last term in (5.5.11) becomes comparable to the first one, with its value defined in terms of the parameters with $(i=2)$

$$
\dot{\phi}=-\frac{H_{0}^{2} \alpha_{2}}{3\left(-\rho_{2}+\delta_{2}+\beta_{2}+\sigma_{2}\right)} .
$$

To ensure the scalar velocity remains continuous at that time we match (5.5.12) and (5.5.13) at $t=t_{1}$. This is possible if

$$
\alpha_{2} \simeq \frac{1}{a^{3}\left(t_{1}\right)} \frac{\alpha_{1}\left(-\rho_{2}+\delta_{2}+\beta_{2}+\sigma_{2}\right)}{\left(-\rho_{1}+\delta_{1}+\beta_{1}+\sigma_{1}\right)} .
$$

In Figure (5.1) we give a schematic behaviour of the field derivative.

The energy constraint (Friedmann) equation for $\mathcal{E}_{j}$ in (3.2.6) becomes

$$
V_{i}=\frac{\dot{\phi}^{2}(t)}{2}\left(-\rho_{i}+2 \delta_{i} \frac{H(t)}{H_{0}}+3 \beta_{i} \frac{H^{2}(t)}{H_{0}^{2}}+4 \sigma_{i} \frac{H^{3}(t)}{H_{0}^{3}}\right) .
$$




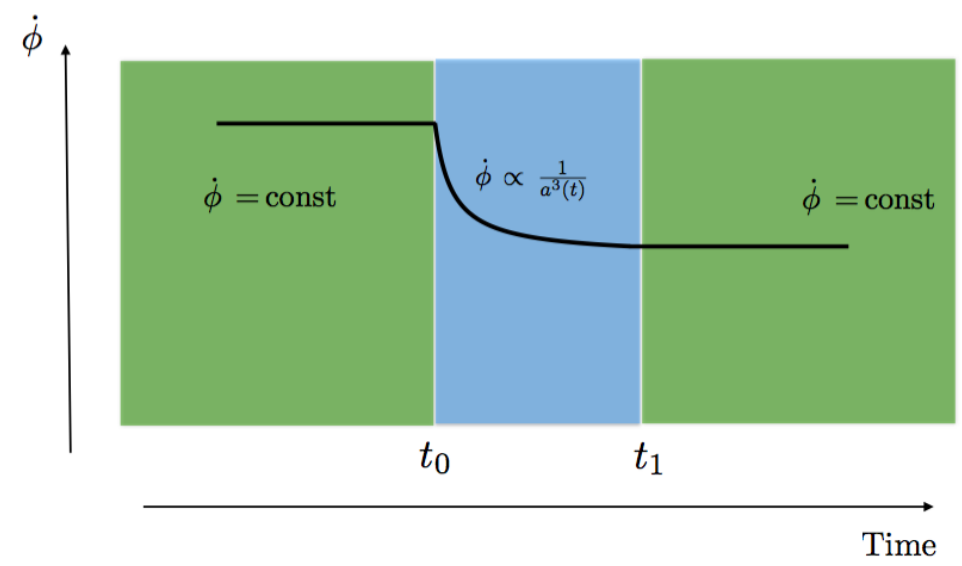

Figure 5.1: Schematic behaviour of the scalar field derivative in our system. Notice the intermediate non-attractor phase $\dot{\phi} \sim 1 / a^{3}$. During the entire inflationary evolution the background geometry corresponds to pure de Sitter space. Image credit: G. Tasinato.

Similarly, the pressure constraint $\mathcal{P}_{j}$ becomes

$$
\begin{aligned}
V_{i} & =\frac{\dot{\phi}(t)}{6}\left\{2 \alpha_{i} H_{0}^{2}+\dot{\phi}(t)\left[3 \rho_{i}+3 \beta_{i} \frac{H^{2}(t)}{H_{0}^{2}}+6 \sigma_{i} \frac{H^{3}(t)}{H_{0}^{3}}+2 \dot{H}(t)\left(\frac{\beta_{i}}{H_{0}^{2}}\right.\right.\right. \\
& \left.\left.\left.+3 \sigma_{i} \frac{H(t)}{h_{0}^{3}}\right)\right]+\ddot{\phi}(t)\left(2 \frac{\delta_{i}}{H_{0}}+4 \beta_{i} \frac{H(t)}{H_{0}^{2}}+6 \sigma_{i} \frac{H^{2}(t)}{H_{0}^{3}}\right)\right\} .
\end{aligned}
$$

In the first phase of the evolution $t<t_{0}$, with $i=1, \dot{\phi}=$ const. and $H(t)=H_{0}$, the constraints (5.5.15) and (5.5.16) correspond to

$$
V_{1}=\frac{H_{0}^{4}}{18} \frac{\alpha_{1}^{2}\left(-\rho_{1}+2 \delta_{1}+3 \beta_{1}+4 \sigma_{1}\right)}{\left(-\rho_{1}+\beta_{1}+\delta_{1}+\sigma_{1}\right)^{2}},
$$

and satisfy (3.2.7) with $\mathcal{E}_{j}=-\mathcal{P}_{j}$, namely

$$
\sum_{j=2}^{5}\left(\mathcal{E}_{j}+\mathcal{P}_{j}\right)=0
$$

In the second phase of the evolution $t>t_{0}$, with $i=2$, the scalar field in (5.5.12) is time-dependent. In order to satisfy both constraints for $H(t)=H_{0}$, we take $V_{2} \rightarrow 0$. The background evolution in (5.5.15) becomes

$$
0=\frac{\dot{\phi}^{2}(t)}{2}\left(-\rho_{2}+2 \delta_{2} \frac{H(t)}{H_{0}}+3 \beta_{2} \frac{H^{2}(t)}{H_{0}^{2}}+4 \sigma_{2} \frac{H^{3}(t)}{H_{0}^{3}}\right) .
$$


It is easy to see that the RHS in (5.5.19) will vanish for $H(t)=H_{0}$, if we choose

$$
\rho_{2}=2 \delta_{2}+3 \beta_{2}+4 \sigma_{2}
$$

Similarly, we find that if we set $V_{2}=0$ and solve (5.5.20) in terms of $\beta_{2}$, giving

$$
\beta_{2}=\frac{1}{3}\left(-2 \delta_{2}+\rho_{2}-4 \sigma_{2}\right)
$$

the pressure constraint in (5.5.16) vanishes $^{1}$ and we have that (3.2.7) is satisfied. This way we ensure that we have a pure de Sitter expansion during the phase where, due to the non-standard kinetic terms, the scalar field is rapidly varying.

After determining the homogeneous background configurations for our system, we now analyze the behaviour of tensor and scalar fluctuations.

\subsubsection{Dynamics of fluctuations}

The dynamics of primordial tensor and scalar fluctuations for inflationary models based on Horndeski theory have been explored in detail in [36]. See instead e.g. [236, 237] for systematic studies on the dynamics of cosmological fluctuations in inflationary models with parameter discontinuities. The dynamics of tensor fluctuations around a FRW background metric with homogeneous scalar profile are described by the quadratic action in (5.3.1). We substitute the definitions in (5.5.5) to find the form of the functions $\mathcal{G}_{T}, \mathcal{F}_{T}$ in (5.3.2). We find

$$
\begin{aligned}
& \mathcal{G}_{T}=\frac{\dot{\phi}^{2}\left(H_{0} \beta_{i}+3 \sigma_{i} H(t)\right)}{6 H_{0}^{3}}, \\
& \mathcal{F}_{T}=-\frac{\dot{\phi}(t)\left(H_{0} \beta_{i} \dot{\phi}(t)-3 \sigma_{i} \ddot{\phi}\right)}{6 H_{0}^{3}} .
\end{aligned}
$$

For the first part of the evolution $t \leq t_{0}$ with $i=1$, we use the expression for $\dot{\phi}=$ const. in (5.5.7), $\ddot{\phi}=0$ and $H(t)=H_{0}$, to find

$$
\mathcal{G}_{T}=\frac{\left(\beta_{1}+3 \sigma_{1}\right)}{6} \frac{\dot{\phi}^{2}}{H_{0}^{2}}=g_{t 1} \frac{\dot{\phi}^{2}}{H_{0}^{2}} \quad \text { and } \quad \mathcal{F}_{T}=-\frac{\beta_{1}}{6} \frac{\dot{\phi}^{2}}{H_{0}^{2}}=f_{t 1} \frac{\dot{\phi}^{2}}{H_{0}^{2}} .
$$

To ensure correct signs for the kinetic term we demand that $\beta_{1}<0, \beta_{1}+3 \sigma_{1}>0 \Rightarrow$ $\sigma_{1}>\left|\beta_{1}\right| / 3>0$.

In the second part of the evolution $t_{0}<t<t_{1}, i=2$, the scalar field is timedependent, as per the expression in (5.5.12). We find that the functions $\mathcal{G}_{T}, \mathcal{F}_{T}$ become $^{2}$

\footnotetext{
${ }^{1}$ The expression for $\mathcal{P}_{i}$ is too large to include in the text. To simplify the expression we substituted for $\dot{H}(t)=-\epsilon(t) H^{2}(t)$ and used that $\epsilon(t)=0$ in de Sitter.

${ }^{2}$ Here we simplified $\mathcal{F}_{T}$ by expressing $\ddot{\phi}$ in terms of the field velocity, $H(t)$ and $\epsilon(t)$, i.e. $\dot{a}(t)=$ $H(t) a(t)$ and $\dot{H}(t)=-\epsilon(t) H^{2}(t)$. Finally we used that during this phase $\alpha_{2} \rightarrow 0, a\left(t_{0}\right)=a_{0}=1$ and that $H(t)=H_{0}, \epsilon=0$ in de Sitter.
} 


$$
\mathcal{G}_{T}=\frac{\left(\beta_{2}+3 \sigma_{2}\right)}{6} \frac{\dot{\phi}^{2}}{H_{0}^{2}}=g_{t 2} \frac{\dot{\phi}^{2}}{H_{0}^{2}} \quad \text { and } \quad \mathcal{F}_{T}=-\frac{\beta_{2}+9 \sigma_{2}}{6} \frac{\dot{\phi}^{2}}{H_{0}^{2}}=f_{t 2} \frac{\dot{\phi}^{2}}{H_{0}^{2}} .
$$

To ensure correct signs for the kinetic term we again demand that $\beta_{2}+3 \sigma_{2}>0$ and $\beta_{2}+9 \sigma_{2}<0$. This is satisfied with $3\left|\sigma_{2}\right|<\beta_{2}<9\left|\sigma_{2}\right|$, where $\beta_{2}>0$ and $\sigma_{2}<0$.

The dynamics for scalar fluctuations are described by the quadratic action in (3.2.16). The find expressions for the functions $\mathcal{G}_{S}, \mathcal{F}_{S}$, in $(3.2 .17)$, we first need to calculate the functions $\Sigma$ and $\Theta$ in (3.2.18) and (3.2.19), in terms of our definitions in (5.5.5). We find

$$
\begin{aligned}
& \Sigma=\dot{\phi}^{2}\left(\frac{1}{2} \rho_{i}-\frac{3}{2} \delta_{i} \frac{H(t)}{H_{0}}-3 \beta_{i} \frac{H^{2}(t)}{H_{0}^{2}}-5 \sigma_{i} \frac{H^{3}(t)}{H_{0}^{3}}\right), \\
& \Theta=\dot{\phi}^{2}\left(\frac{\delta_{i}}{6 H_{0}}+\frac{\beta_{i} H(t)}{2 H_{0}^{2}}+\frac{\sigma_{i} H^{2}(t)}{H_{0}^{3}}\right) .
\end{aligned}
$$

The expressions for $\mathcal{G}_{S}, \mathcal{F}_{S}$ are long winded so we won't display them. We go directly to expressing their behaviour for the first part of the evolution $t \leq t_{0}$ with $i=1$, in the same way as we did earlier for tensors. To simplify things we take that $\delta_{1}=0$. We find

$$
\begin{aligned}
& \mathcal{G}_{S}=\frac{\left(\beta_{1}+3 \sigma_{1}\right)\left[3 \beta_{1}^{2}+\rho_{1}\left(\beta_{1}+3 \sigma_{1}\right)+2 \sigma_{1}\left(4 \beta_{1}+3 \sigma_{1}\right)\right]}{18\left(\beta_{1}+2 \sigma_{1}\right)^{2}} \frac{\dot{\phi}^{2}}{H_{0}^{2}}=g_{s 1} \frac{\dot{\phi}^{2}}{H_{0}^{2}}, \\
& \mathcal{F}_{S}=\frac{\left(2 \beta_{1}+3 \sigma_{1}\right)^{2}}{18\left(\beta_{1}+2 \sigma_{1}\right)} \frac{\dot{\phi}^{2}}{H_{0}^{2}}=f_{s 1} \frac{\dot{\phi}^{2}}{H_{0}^{2}} \quad \text { and } \quad f_{s 1}=\frac{\left(f_{t 1}-g_{t 1}\right)^{2}}{2 g_{t 1}-f_{t 1}},
\end{aligned}
$$

where in the last step we related $f_{s 1}$ to the tensor parameters $g_{t 1}, f_{t 1}$ in (5.5.23). We ensure correct signs for the kinetic terms by demanding $\beta_{1}+2 \sigma_{1}>0 \Rightarrow \sigma_{1}>\left|\beta_{1}\right| / 2$ where, as before, $\beta_{1}<0$ and $\sigma_{1}>0$. By combining the conditions for stability in the scalar and tensor sectors, we find the simple condition $\beta_{1}+3 \sigma_{1}>-\beta_{1} / 2$. Furthermore, condition $g_{s 1}>0$ can easily be satisfied by making appropriate choices for the parameter $\rho_{1}$.

For the second part of the evolution $t_{0}<t<t_{1}, i=2$, we find

$$
\begin{aligned}
& \mathcal{G}_{S}=\frac{\left(\beta_{2}+3 \sigma_{2}\right)\left(2 \beta_{2}+\delta_{2}+3 \sigma_{2}\right)}{2\left(3 \beta_{2}+\delta_{2}+6 \sigma_{2}\right)} \frac{\dot{\phi}^{2}}{H_{0}^{2}}=g_{s 2} \frac{\dot{\phi}^{2}}{H_{0}^{2}}, \\
& \mathcal{F}_{S}=\frac{-2 \beta_{2}^{2}+\delta_{2}\left(\beta_{2}+9 \sigma_{2}\right)+3 \sigma_{2}\left(\beta_{2}+3 \sigma_{2}\right)}{6\left(3 \beta_{2}+\delta_{2}+6 \sigma_{2}\right)} \frac{\dot{\phi}^{2}}{H_{0}^{2}}=f_{s 2} \frac{\dot{\phi}^{2}}{H_{0}^{2}}
\end{aligned}
$$

where we used the expression for $\rho_{2}$ in (5.5.20) to simplify $g_{s 2}$. We also find the following relations

$$
\begin{aligned}
& g_{s 2}=\frac{3}{5}\left(f_{s 2}+f_{t 2}+5 g_{t 2}\right) \\
& \rho_{2}=-\frac{f_{t 2}\left(f_{s 2}+f_{t 2}\right)+19 g_{t 2}\left(f_{s 2}+f_{t 2}\right)+60 g_{t 2}^{2}}{f_{s 2}+f_{t 2}}
\end{aligned}
$$


As we have already chosen $\beta_{2}>0$, and $\sigma_{2}<0$, we demand that the nominator and denominator of $f_{s 2}$ are negative which guarantees $f_{s 2}>0$, by appropriately choosing values for $\delta_{2}<0$. This also implies that $\rho_{2}<0$. Finally we can see from relation (5.5.28) that $g_{s 2}>0$ as long as $f_{t 2}, g_{t 2}, f_{s 2}>0$.

In this work we are only interested to study the enhancement of tensors during the second part of the evolution. In subsequent parts of the evolution $\left(t \geq t_{1}\right)$ the behaviour of the fluctuations and therefore the dynamics of the system, that we are currently studying, will change in order to accommodate a smooth transition to slow-roll inflation. Consequently, we expect that the parameter $\alpha_{2}$ will become important. We leave these considerations for another work.

Next, we analyse an explicit choice of quantities within the available parameter space which satisfies the aforementioned stability conditions and discuss the corresponding physical consequences.

\subsection{Enhancement of fluctuations}

With all of the above in place we can now examine the physical consequences of our model. We find that in the second part of the phase, both the scalar and tensor fluctuations can be enhanced.

In the first part of the phase $t<t_{0}$, using (5.5.7), we have that the effective potential for tensor fluctuations, is given by

$$
z_{T}=\frac{a}{2}\left(\mathcal{G}_{T} \mathcal{F}_{T}\right)^{\frac{1}{4}}=\frac{a \dot{\phi}}{2 H_{0}}\left(g_{t 1} f_{t 1}\right)^{\frac{1}{4}}=-a(t) \frac{\alpha_{1} H_{0}\left(f_{t 1} g_{t 1}\right)^{\frac{1}{4}}}{6\left(-\rho_{1}+\delta_{1}+\beta_{1}+\sigma_{1}\right)},
$$

while the effective potential for scalars is given by

$$
z_{S}=\sqrt{2} a\left(\mathcal{G}_{S} \mathcal{F}_{S}\right)^{\frac{1}{4}}=\frac{\sqrt{2} a \dot{\phi}}{H_{0}}\left(g_{s 1} f_{s 1}\right)^{\frac{1}{4}}=-a(t) \frac{\sqrt{2} \alpha_{1} H_{0}\left(f_{s 1} g_{s 1}\right)^{\frac{1}{4}}}{3\left(-\rho_{1}+\delta_{1}+\beta_{1}+\sigma_{1}\right)} .
$$

In the second part of the phase $t_{0}<t<t_{1}$, using (5.5.12), we have

$$
\tilde{z}_{T}=\frac{a}{2}\left(\mathcal{G}_{T} \mathcal{F}_{T}\right)^{\frac{1}{4}}=\frac{a \dot{\phi}}{2 H_{0}}\left(g_{t 2} f_{t 2}\right)^{\frac{1}{4}}=-\frac{1}{a^{2}(t)} \frac{\alpha_{1} H_{0}\left(f_{t 2} g_{t 2}\right)^{\frac{1}{4}}}{6\left(-\rho_{1}+\delta_{1}+\beta_{1}+\sigma_{1}\right)},
$$

and

$$
\tilde{z}_{S}=\sqrt{2} a\left(\mathcal{G}_{S} \mathcal{F}_{S}\right)^{\frac{1}{4}}=\frac{\sqrt{2} a \dot{\phi}}{H_{0}}\left(g_{s 2} f_{s 2}\right)^{\frac{1}{4}}=-\frac{1}{a^{2}(t)} \frac{\sqrt{2} \alpha_{1} H_{0}\left(f_{s 2} g_{s 2}\right)^{\frac{1}{4}}}{3\left(-\rho_{1}+\delta_{1}+\beta_{1}+\sigma_{1}\right)} .
$$

It is now clear that, with the scalar velocity behaving like $\dot{\phi} \propto a^{-3}$, we realize the condition in (5.4.15) and the tensor duality indicated in (5.4.13). Therefore, we expect that modes leaving the horizon during the time interval $t_{0}<t<t_{1}$ will 
receive an exponential enhancement proportional to $a^{6}$ (recall $a \sim \exp \left[H_{0} t\right]$ in de Sitter). Please note that since, during this phase, fluctuations evolve beyond the Hubble horizon, the power spectra should be evaluated at the end of this phase at $t=t_{1}$, as opposed to taking the limit $t \rightarrow 0$, which is when we recover standard slow-roll inflation dynamics.

Using the above, we find that the tensor power spectrum at the end of the second part of the evolution can be amplified with respect to the one during the preceding slow-roll phase, by the following amount

$$
\left.\frac{P_{\tilde{h}}}{P_{h}}\right|_{t=t_{1}}=\left.\left(\frac{z_{T}}{\tilde{z}_{T}}\right)^{2}\right|_{t=t_{1}}=a\left(t_{1}\right)^{6}\left(\frac{g_{t 1} f_{t 1}}{g_{t 2} f_{t 2}}\right)^{\frac{1}{2}}=a\left(t_{1}\right)^{6}\left(\frac{\beta_{1}\left(\beta_{1}+3 \sigma_{1}\right)}{\left(\beta_{2}+3 \sigma_{2}\right)\left(\beta_{2}+9 \sigma_{2}\right)}\right)^{\frac{1}{2}},
$$

where we have used (5.5.14) to express the scale factor at $t=t_{1}$, as

$$
a^{6}\left(t_{1}\right) \simeq\left(\frac{\alpha_{1}}{\alpha_{2}}\right)^{2} \frac{\left(-\rho_{2}+\delta_{2}+\beta_{2}+\sigma_{2}\right)^{2}}{\left(-\rho_{1}+\delta_{1}+\beta_{1}+\sigma_{1}\right)^{2}} .
$$

It is worth emphasizing that we can enhance the amplitude of the fluctuations while maintaining a scale invariant statistics in the non-attractor regime ${ }^{3}$. This behaviour can be particularly interesting to build models with an enhanced tensor power spectrum detectable at interferometer scales. See also [238] for a scenario able to amplify the tensor-to-scalar ratio $r$ at small scales, by reducing the size of the spectrum of scalar fluctuations.

Similarly to the tensor fluctuations, scalar fluctuations grow during the nonattractor regime since the scalar pump field $\tilde{z}_{S}$ has a similar structure to $\tilde{z}_{T}$. Using Wands' duality, the scalar power spectrum reads

$$
\left.\frac{P_{\tilde{\mathcal{R}}}}{P_{\mathcal{R}}}\right|_{t=t_{1}}=\left.\left(\frac{z_{S}}{\tilde{z}_{S}}\right)^{2}\right|_{t=t_{1}}=a\left(t_{1}\right)^{6}\left(\frac{g_{s 1} f_{s 1}}{g_{s 2} f_{s 2}}\right)^{\frac{1}{2}}
$$

where $g_{s 1}, f_{s 1}, g_{s 2}, f_{s 2}$ were defined in (5.5.26), (5.5.27) and (5.5.28). Again, we do not display the evaluation of the terms inside the brackets as the expression is too long winded.

\subsection{Numerical example}

Let us now look at a specific example. We define the following set of parameters

$$
\left(\alpha_{i}, \beta_{i}, \delta_{i}, \rho_{i}, \sigma_{i}\right)= \begin{cases}(1,-1,0,1,2.5) & t<t_{0}, \\ \left(10^{-3}, 1.5,-4,-4.3,-0.2\right) & t>t_{0} .\end{cases}
$$

\footnotetext{
${ }^{3}$ At scales corresponding to the modes leaving the horizon slightly before the transition to nonattractor regime, we expect peaks or features in the fluctuation power spectra, whose study require more careful numerical investigations, see e.g. [221].
} 
For this choice, the corresponding field velocity $\dot{\phi}$ and the amplification of $\left|h_{i j}\right|$, using the super-horizon expression in (5.3.10), are shown in Figure 5.2. However, we would like to note that equation (5.3.10) can be only used as a rough indicator of the enhancement of the fluctuations as it relies on strict super-horizon limit $k=0$ (see for example [221] for a detailed discussion on this issue). On the other hand, to obtain a more accurate estimate on the enhancement we use the expressions based on the duality in (5.6.5) and (5.6.7) with the parameter choices in (5.7.1).

From this we find that the tensor power spectrum in (5.6.5) can be enhanced by a factor of $\left(z_{T} / \tilde{z}_{T}\right)^{2} \approx 5 \times 10^{7}$, while the scalar power spectrum in (5.6.5) can be enhanced by a factor of $\left(z_{S} / \tilde{z}_{S}\right)^{2} \approx 5 \times 10^{6}$. At the same time we ensure that the system obeys all the stability constraints for both scalars and tensors ${ }^{4}$.

We can also use these parameter choices to investigate the behaviour of the scalar velocity and the tensor amplitudes. We find, using (5.5.7), that the velocity of the scalar field at time $t \leq t_{0}$ becomes

$$
\left|\frac{\dot{\phi}}{H_{0}^{2}}\right|=\left|-\frac{\alpha_{1}}{3\left(-\rho_{1}+\delta_{1}+\beta_{1}+\sigma_{1}\right)}\right|=|-0.67| .
$$

For the second part of the evolution, we use that the number of e-folds can be written as $\mathrm{d} N=H \mathrm{~d} t=\mathrm{d} \ln a$, to express the scale factor ${ }^{5}$ as $a(t) \sim \exp \left[\int \mathrm{d} t H\right]=$ $\exp [N(t)]$. As the second part of the phase ends at $t=t_{1}$, we solve for $N$ using $(5.5 .14)$

$$
a^{3}\left(t_{1}\right)=e^{3 N} \simeq \frac{\alpha_{1}}{\alpha_{2}} \frac{\left(-\rho_{2}+\delta_{2}+\beta_{2}+\sigma_{2}\right)}{\left(-\rho_{1}+\delta_{1}+\beta_{1}+\sigma_{1}\right)} \Rightarrow e^{3 N} \simeq 3200 \Rightarrow N \simeq 2.7
$$

From this we find that the enhancement of the scalar and tensor fluctuations is mainly controlled by the duration of the second part of the evolution, namely by the ratio $\alpha_{1} / \alpha_{2}$. We find this is the dominant contribution towards the number of e-folds with $\alpha_{1} / \alpha_{2} \approx 1000 \Rightarrow N \approx 2.3$. The rest of the model parameters contribute only a factor of $\approx 3$ which gives a negligible contribution of $\approx 0.36$ towards the number of e-folds. Therefore, the role of these parameters is simply to ensure that the stability conditions are satisfied for both sectors, as they do not add much else into the model.

We can express the velocity of the field at $t_{0}<t<t_{1}$, using (5.5.11), in terms of the number of e-folds $a(t) \sim \exp [N]$, giving

\footnotetext{
${ }^{4}$ Interestingly, we learn that scalar fluctuations are less enhanced than tensor ones. This could be useful when building more realistic scenarios of our mechanism, to avoid constraints from excessive primordial black hole production.

${ }^{5}$ The usual convention, found in the literature is to adopt a minus sign so that $N$ is large and decreasing as we approach the end of inflation (i.e. while the scale factor increases). Here we are interested to find how many e-folds the second part of the evolution lasts for, where $N=0$ corresponds to the transition point, so we adopt sign convention opposite to that of the literature with $a \sim \exp [N]$.
} 


$$
\left|\frac{\dot{\phi}}{H_{0}^{2}}\right|=\left|-0.208 e^{-3 N}\left(3.199+\frac{e^{3 N}}{1000}\right)\right| .
$$

We use the above to plot the behaviour of the scalar field velocity $\dot{\phi}$ during the transition. This is displayed on the left graph in Figure (5.2). The scalar field has constant velocity until the time $t=t_{0}$ where its velocity starts decreasing, lasting $\Delta N=2.7$ e-folds. During this time we have, using (5.5.12), that $\dot{\phi} \propto \exp [-3 N]$.

To find the behaviour of the tensor amplitudes during this time, we make use of the relationship between the tensor field and its dual in (5.4.5). Using (5.3.10), we have

$$
h_{i j}=\frac{\tilde{z}_{T}}{z_{T}} \tilde{h}_{i j}=\frac{\tilde{z}_{T}}{z_{T}}\left(\mathcal{C}_{1}+\mathcal{C}_{2} \int_{a_{0}}^{a_{1}} \mathrm{~d} t \frac{c_{T}}{H_{0} a^{2}(t) \tilde{z}_{T}^{2}(t)}\right)
$$

where we have rewritten the integrand as shown below

$$
\int_{y_{0}}^{y_{1}} \mathrm{~d} y \frac{1}{\tilde{z}_{T}^{2}(y)}=\int_{t_{0}}^{t_{1}} \mathrm{~d} t \frac{c_{T}}{a(t) \tilde{z}_{T}^{2}(t)}=\int_{a_{0}}^{a_{1}} \mathrm{~d} a \frac{c_{T}}{H_{0} a^{2}(t) \tilde{z}_{T}^{2}(t)} .
$$

In the second step we expressed the integrand with respect to the coordinate time and in the last step we used $a(t)=\exp [H t] \Rightarrow \mathrm{d} a=H a \mathrm{~d} t$ to express it in terms of the scale factor. We can use the expression for $\tilde{z}_{T}$ in (5.6.3) and the relations for $f_{t 1}$ and $f_{t 2}$ in (5.5.24) to evaluate the integrand. We find

$$
\int_{a_{0}}^{a_{1}} \mathrm{~d} a \frac{c_{T}}{H_{0} a^{2}(t) \tilde{z}_{T}^{2}(t)}=\frac{64000 \sqrt{3}\left(-1+e^{3 N}\right)}{3199+e^{3 N}},
$$

where we substituted for $a\left(t_{1}\right)=\exp [3 N]$. Therefore, in the second part of the evolution we have

$$
h_{i j}=\frac{\tilde{z}_{T}}{z_{T}} \tilde{h}_{i j}=10^{-7}\left(1+\frac{64000 \sqrt{3}\left(-1+e^{3 N}\right)}{3199+e^{3 N}}\right),
$$

where we have used the approximate value $\left(z_{T} / \tilde{z}_{T}\right)^{2} \approx 10^{7}$, meaning $\left(\tilde{z}_{T} / z_{T}\right)^{2} \approx 10^{-7}$ and have set $\mathcal{C}_{2}=\mathcal{C}_{1}\left(H_{0} / c_{T}\right)$. The behaviour of $\left|h_{i j}\right|$ is shown in the right graph of Figure (5.2). As expected, the tensor fluctuations are enhanced, starting at the transition point $N=0$ and lasting for 2.7 e-folds.

Finally, we quickly remark on the speed of propagation for tensor and scalar fluctuations, in our model. The kinetic functions for both scalars and tensors are characterised by the relations $\mathcal{G}_{S} \neq \mathcal{F}_{S}$ and $\mathcal{G}_{T} \neq \mathcal{F}_{T}$, which lead to fluctuations propagating with non-trivial speeds. These are given by 

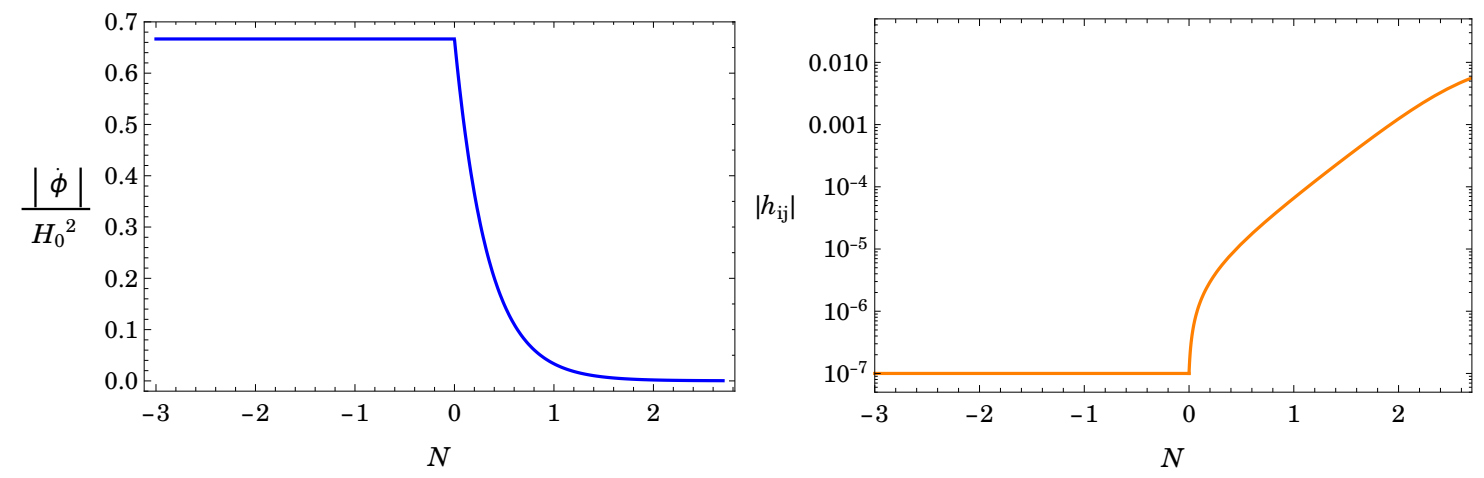

Figure 5.2: The behaviour of $\dot{\phi}$ and $\left|h_{i j}\right|$ through the transition to the non-attractor phase where we have used the expressions (5.5.12) and (5.3.10), respectively. On the right, we have set $\mathcal{C}_{2}=\mathcal{C}_{1}\left(H_{0} / c_{T}\right)$ with $\mathcal{C}_{1}=10^{-7}$ in (5.3.10) to show the evolution of $\left|h_{i j}\right|$. For both plots we have the following choice of parameters: $\alpha_{1}=$ $1, \beta_{1}=-1, \sigma_{1}=2.5, \rho_{1}=1, \delta_{1}=0, \alpha_{2}=10^{-3}, \beta_{2}=1.5, \sigma_{2}=-0.2, \delta_{2}=$ $-4, \rho_{2}=-4.3$ where $N=0$ corresponds to the transition point. In these plots the non-attractor phase lasts approximately $\Delta N \simeq 2.7$ e-folds.

$$
c_{S}^{2}= \begin{cases}f_{s 1} / g_{s 1} & t<t_{0} \\ f_{s 2} / g_{s 2} & t>t_{0}\end{cases}
$$

and

$$
c_{T}^{2}= \begin{cases}f_{t 1} / g_{t 1} & t<t_{0} \\ f_{t 2} / g_{t 2} & t>t_{0} .\end{cases}
$$

Using our parameter choices, we find that during both phases of the inflationary evolution, $t<t_{0}$ and $t>t_{0}$, the speed of propagation for the fluctuations is less than unity, i.e. $c_{s}^{2}<1$ and $c_{T}^{2}<1$. In particular, we have $c_{T}^{2} \approx 0.15$ during $t<t_{0}$ and $c_{T}^{2} \approx 0.33$ during $t>t_{0}$. Similarly, we have $c_{s}^{2} \approx 0.69$ during $t<t_{0}$ and $c_{s}^{2} \approx 0.89$ during $t>t_{0}$.

\subsection{Outlook}

In this work we discussed a new mechanism to amplify tensor fluctuations during single field inflation, by exploiting a phase of non-attractor evolution. We have identified the necessary condition for amplifying the tensor spectrum at super-horizon scales, in terms of (5.3.8), which decreases with time during a phase of the inflationary evolution. Through this process the would-be decaying tensor mode gets enhanced and increases the size of tensor fluctuations. We determined a criterium, which we dub tensor duality, which allows us to analytically estimate the statistical properties of the amplified tensor fluctuations during the non-attractor era. We then built and investigated in detail a concrete model of kinetically driven inflation able 
to satisfy our conditions and analytically determined the properties of the enhanced spectrum of tensor modes in this set-up. Much work is left for the future:

- Our concrete scenario is based on G-inflation, since we need a non-trivial kinetic mixing between scalar and metric to realise our mechanism. It will be interesting to understand whether other realisations can exist, for example by means of sudden changes in the tensor sound speed due to effects of new heavy physics or string theory, as in [239].

- The quadratic tensor action we obtained in our system is distinct from the one of single-field inflation with standard kinetic terms, but a sequence of conformal and disformal transformations can recast it in standard form [135]. In Appendix A.13 we show that our system, during the non-attractor era, can be disformally related to a rapidly contracting universe. It will be interesting to further explore the physical implications of disformal transformations during non-attractor regimes.

- We provided evidence that the spectrum of tensor fluctuations can be nonGaussian, besides being enhanced. It will be important to analytically study in more details the amplitude and shape of non-Gaussianity of tensor modes in our set-up.

- Finally, it will be important to build a complete and realistic scenario (based on G-inflation or on other theories) able to sufficiently amplify tensor modes at interferometer scales and study prospects for the detectability of the stochastic primordial tensor background and its non-Gaussianity.

Finally, tensor non-Gaussianity, is an observable that can be useful for discriminating among primordial and astrophysical stochastic gravitational wave background detectable with interferometers [240]. Tensor non-Gaussianity is also an important observable for characterizing the primordial stochastic gravitational wave background at CMB scales and have been explored in other contexts, see e.g. $[200,203,241]$. We plan to examine this subject, in the tensor-dual regime, in the next Chapter. 


\section{Non-Gaussianity in non-attractor inflation}

\subsection{Abstract}

We investigate primordial tensor non-Gaussianity in single field inflation, during a phase of non-attractor evolution when the spectrum of primordial tensor modes can be enhanced to a level detectable at interferometer scales. Making use of a tensor duality we introduced in [8], we analytically compute the full bispectrum of primordial tensor fluctuations during the non-attractor era. During this epoch the shape of the tensor bispectrum is enhanced in the squeezed limit, its amplitude can be amplified with respect to slow-roll models and tensor non-Gaussianity can exhibit a scale dependence distinctive of our set-up. We prove that our results do not depend on the frame used for the calculations.

\subsection{Introduction}

The possibility to directly detect a stochastic background of primordial tensor modes with gravitational wave experiments would offer new ways to probe the physics of inflation. Such an opportunity would allow us to probe a much larger range of frequency scales than what can be tested with CMB physics. Various scenarios have been proposed for enhancing the primordial tensor spectrum at interferometer scales: from coupling the inflation to additional fields, whose dynamics are characterised by instabilities that amplify the tensor spectrum (see e.g. [114, 183-191, 193-204, $242]$ ), to models that break space-time symmetries during inflation, leading to a blue spectrum for primordial tensor modes (see e.g. [205-217]). See the general discussion in [218].

Here we consider scenarios of single field inflation in which the inflationary slowroll expansion is briefly interrupted in which case the inflationary expansion undergoes a brief phase of non-attactor dynamics that amplify the tensor modes. Non-attractor cosmological evolution is known to enhance the scalar sector of fluctuations, for example during ultra-slow roll or in constant roll inflationary systems [156-164]: this property has been exploited in models producing primordial black holes in single-field inflation (see e.g. [165-167] for reviews and [168-176] for specific models). 
During this time the amplitude of the would-be decaying mode becomes amplified (while the growing mode has a constant amplitude) instead of being suppressed at superhorizon scales, which is the usual case for slow-roll inflation. This can enhance the tensor spectrum which may be detectable at interferometer scales. This type of system was discussed in the previous Chapter [8] where it was demonstrated that one can maintain analytic control over the dynamics of the tensor modes during the short period that slow-roll inflation is violated, by employing a tensor duality [178] which maps the evolution of tensor fluctuations during the non-attractor phase to the dynamics of tensor fluctuations in a slow-roll phase of expansion. We use the duality to obtain an analytic control on the physics of tensor modes during the phase of non-attractor evolution, even if we are far from a slow-roll approximation and to analytically compute the properties of tensor non-Gaussianity during the non-attractor phase. This enables us to derive analytic expressions for the tensor bispectra, which would be mostly the focus of this Chapter.

Tensor non-Gaussianity is an interesting observable which can help to characterise and distinguish different scenarios of inflation that enhance tensor modes at small or large frequency scales (see e.g. [64,137,141,142,200,203, 243-251] and the review in Section 5 of [240] for more a comprehensive reference list).

In what follows, we show that the tensor bispectrum can be enhanced if slowroll inflation is violated for short period of time, as in our setup. We find that its shape is amplified in the squeezed limit, as during this time the bispectrum does not satisfy Maldacena's consistency relation [64], which simply states that single-field inflation cannot result to large non-Gausianity at the squeezed limit. We find that the bispectrum, in our case, is parametrically amplified with respect to the standard slow-roll scenario. Additionally, we find the bispectrum is scale dependent, in such a way that it could help to distinguish it, in the future, from other frameworks that produce large tensor non-Gaussianity. Finally, we show that our results remain the same after applying a disformal plus a conformal transformation to our system. These transformations, at quadratic level in a perturbative expansion in tensor fluctuations, render the system identical to Einstein gravity minimally coupled with a scalar field $[135,136]$. On the other hand, as we shall discuss, at cubic level in a perturbative expansion tensor interactions include terms as $\dot{h}_{i j}^{3}$ which cannot be associated with contributions of standard Einstein gravity.

In Figure (6.1) we show how, in our system, the spectrum of the superhorizon modes can be amplfied to enter within the sensitivity curves for GW detectors, using formula (5.6.5) and assuming, for simplicity, instantaneous transitions between attractor and non-attractor eras. The Figure in (6.1) is only indicative, because it does not take into account the transition phases during different epochs and, above all, does not take into consideration additional model-dependent constraints from amplification of scalar modes.

\subsection{The two-point function}

The behaviour of the system during the first and second part of the evolution were analysed in the previous Chapter. The action in (5.4.2) was given in terms of 

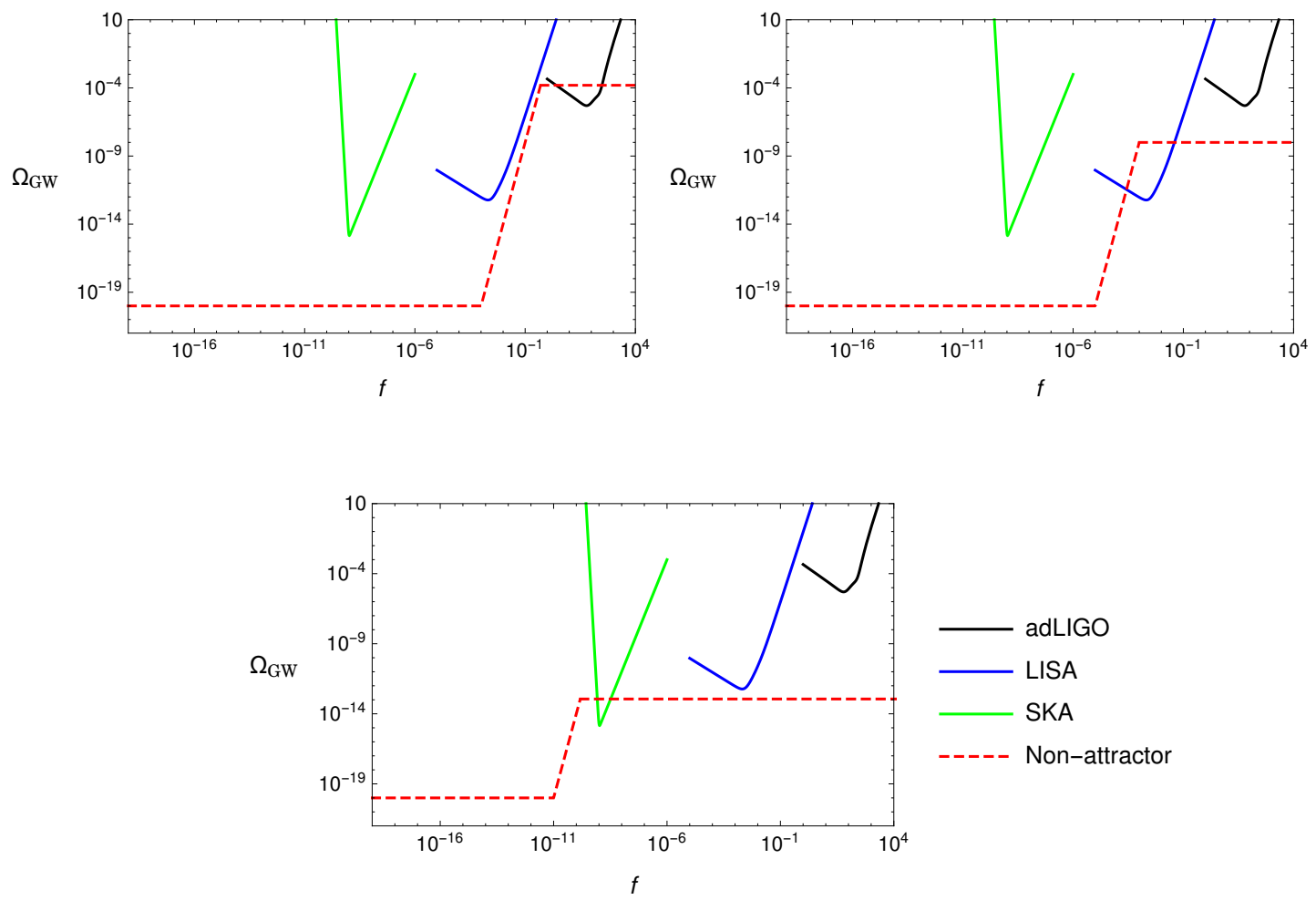

Figure 6.1: We show qualitatively how primordial tensor modes get amplified during a non-attractor phase. They can contribute to the $G W$ energy density $\Omega_{G W}$ and thus enter within the sensitivity curves for $G W$ detectors in their appropriate frequency ranges (expressed in $\mathrm{Hz}$ ). We model inflation as a pure de Sitter phase, during which a short period of non-attractor evolution occurs - whose starting time and duration depend on the model one considers - enhancing the tensor spectrum. We use formula (5.6.5) and assume for simplicity instantaneous transitions between attractor and non-attractor eras. Our conventions for the definition of the $G W$ energy density $\Omega_{G W}$ are the same as in [6]. Image credit: Plots by Cari Powell.

the canonically normalized variable $q_{i j}$ in (5.4.1). From this we get the evolution equation for tensor fluctuations in Fourier space is given by the standard expression

$$
q^{\prime \prime}+\left(k^{2}-\frac{z_{T}^{\prime \prime}}{z_{T}}\right) q=0,
$$

where $q=q(\mathbf{k}, y)$. Using (5.4.4) and the expression for $z_{T}$ in (5.3.8), we find

$$
q^{\prime \prime}+\left(k^{2}-\frac{2}{y^{2}}\right) q=0
$$

which has exact solution

$$
q=-\frac{1}{\sqrt{2 k}}\left(1-\frac{i}{k y}\right) e^{-i k y}
$$




\section{NON-GAUSSIANITY IN NON-ATTRACTOR INFLATION}

The solution $q$ at small scales becomes

$$
\lim _{|k y| \rightarrow \infty} q=-\frac{1}{\sqrt{2 k}} e^{-i k y}
$$

Using (5.4.1), the tensor perturbations can be expressed as

$$
h=\frac{q}{z_{T}}=-\frac{e^{-i k y}\left(1-\frac{i}{k y}\right) \sqrt{2} H y}{\sqrt{\mathcal{F}_{T} c_{T} k^{3}}}=\frac{i \sqrt{2} H}{\sqrt{\mathcal{F}_{T} c_{T} k^{3}}}(1+i k y) e^{-i k y},
$$

which is the same with the solution in [252] (see eq (11)) during slow-roll inflation. Note that we evaluated $z_{T}$ in terms of $y$ by using that $a=-\left[H\left(y / c_{T}\right)\right]^{-1}$ in de Sitter. At large scales, the solution becomes

$$
\lim _{|k y| \rightarrow 0} h=-\frac{i \sqrt{2} H}{\sqrt{\mathcal{F}_{T} c_{T} k^{3}}} .
$$

The tensor two-point function was defined in (2.3.11) and (2.3.12). Using (2.3.23), we evaluate the tensor powerspectrum to be

$$
P_{h}=\frac{k^{3}}{2 \pi^{2}} P_{i j, i j}=\frac{k^{3}}{2 \pi^{2}} \frac{2 H^{2}}{\mathcal{F}_{T} c_{T} k^{3}} p_{i j}^{s}(\mathbf{k}) p_{i j}^{s}(\mathbf{k})=\frac{2}{\pi^{2}} \frac{H^{2}}{\mathcal{F}_{T} c_{T}} .
$$

During the second part of the evolution $\left(t_{0}<t<t_{1}\right)$ the tensor kinetic term evolves as $\mathcal{F}_{T} \propto a^{-6}$, which is heavily-time dependent. This can be easily seen by substituting (5.5.12) in (5.5.24). Therefore the amplitude of the tensor spectrum grows on super-horizon scales and it has to be evaluated at the end of the nonattractor era.

Using (5.5.13) and (5.5.24), we define

$$
\mathcal{F}_{T}(y)=\mathcal{F}_{T}^{(0)}\left(\frac{y}{y_{0}}\right)^{6}, \quad \mathcal{G}_{T}(y)=\mathcal{G}_{T}^{(0)}\left(\frac{y}{y_{0}}\right)^{6},
$$

where we have absorbed constant parameters in

$$
\mathcal{F}_{T}^{(0)}=\frac{f_{t}}{\Lambda^{2}} \frac{H_{0}^{2} \alpha_{1}^{2}}{9\left(-\rho_{1}+\delta_{1}+\beta_{1}+\sigma_{1}\right)^{2}}, \quad \mathcal{G}_{T}^{(0)}=\frac{g_{t}}{\Lambda^{2}} \frac{H_{0}^{2} \alpha_{1}^{2}}{9\left(-\rho_{1}+\delta_{1}+\beta_{1}+\sigma_{1}\right)^{2}}
$$

therefore, $c_{T}=\left(\frac{f_{T}}{g_{T}}\right)^{\frac{1}{2}}$ and we have $y_{0}=-\frac{c_{T}}{H_{0}}$. In the non-attractor era the solution in (6.3.5) takes the form

$$
h=\frac{i \sqrt{2} H}{\sqrt{\mathcal{F}_{T}^{(0)} c_{T} k^{3}}}(1+i k y) e^{-i k y}\left(\frac{y_{0}}{y}\right)^{3} .
$$


From this we find the power spectrum in, (6.3.7), becomes

$$
P_{h}=\frac{2}{\pi^{2}} \frac{H_{0}^{2}}{F_{T}^{(0)} c_{T}}\left(\frac{y_{0}^{6}}{y_{\text {end }}^{6}}\right)
$$

\subsection{Tensor non-Gaussianity in non-attractor in- flation}

Experiments, so far, have shown that the bispectrum for scalar fluctuations is Gaussian. On the other hand if, in the future, we make a detection of large tensor non-Gaussianity with interferometers, then it is likely that it will be of primordial origin, since astrophysical stochastic gravitational backgrounds, which are usually formed by many unresolved sources, are likely to be Gaussian due to the central limit theorem.

The bispectrum has many important properties that can help us distinguish between different inflationary models or other primordial sources that can produce an amplified tensor spectrum at interferometer scales. Some of these are the shape, scale dependence and its value at the squeeze limit [136].

In this Section we study the tensor bispectrum resulting during the short regime where slow-roll inflation is violated. Our starting point is the Horndeski cubic action for tensor perturbations, expanded around an FRW background

$$
S_{T}^{(3)}=\frac{1}{4} \int \mathrm{d} \tau \mathrm{d}^{3} x\left[a^{2} \mathcal{F}_{T}\left(h_{j l} h_{i k}-\frac{1}{2} h_{k l} h_{i j}\right) \partial_{k} \partial_{l} h_{i j}+\frac{X \phi^{\prime} G_{5 X}}{12} h_{i j}^{\prime} h_{j k}^{\prime} h_{k i}^{\prime}\right]
$$

where prime denotes differentiation with respect to the conformal time $\tau$ and $M_{P l}=$ 1. The first term in (6.4.1) contains only spatial derivatives and for $\mathcal{F}_{T}=1$ it is proportional to the Einstein-Hilbert term expanded to cubic order. For convenience we dub it $S_{T(\mathrm{GR})}^{(3)}$. The last contribution, which contains three time-derivatives, is unique to the Horndeski theory so we dub it $S_{T(\text { new })}^{(3)}$. Contributions with $\left(h_{i j}^{\prime}\right)^{3}$ usually appear at the cubic Lagrangian for gravitational square curvature terms, i.e. from the Weyl squared tensor.

The action in (6.4.1) was studied in the slow-roll case in $[43,252]$, where they found that the "GR" term is enhanced in the squeezed limit and the "new" term peaks in equilateral configurations. In what follows we study tensor non-Gaussianity during the second part of the evolution where slow-roll conditions are violated.

As in the previous Sections, we choose to work in an exact de Sitter background with $H=H_{0}=$ const. We first compute the "GR" contribution. Relation (2.5.6) becomes 


$$
\begin{aligned}
\left\langle h^{3}\left(\tau_{e}\right)\right\rangle & =2 \operatorname{Re}\left\{-i h_{t m}\left(\mathbf{k}_{1}, \tau_{e}\right) h_{n r}\left(\mathbf{k}_{2}, \tau_{e}\right) h_{p v}\left(\mathbf{k}_{3}, \tau_{e}\right)\right. \\
& \times \int_{-\infty(1-i \epsilon)}^{\tau_{e}} \mathrm{~d} \tau \int \mathrm{d}^{3} x\left(-\frac{1}{4} a^{2} \mathcal{F}_{T}\right)\left[h^{j l}(\mathbf{x}, \tau) h^{i k}(\mathbf{x}, \tau)\right. \\
& \left.\left.-\frac{1}{2} h^{k l}(\mathbf{x}, \tau) h^{i j}(\mathbf{x}, \tau)\right] \partial_{k} \partial_{l} h_{i j}(\mathbf{x}, \tau)\right\},
\end{aligned}
$$

where the initial time $\tau_{0}$ is taken to be some early time in the far past when perturbations are still within the horizon $\tau_{0} \rightarrow-\infty$. The upper limit $\tau_{e}=\tau_{\text {end }}$ denotes the end of the second part of the evolution (in the usual case of slow-roll inflation, it would denote the end of inflation and therefore $\tau_{e} \rightarrow 0$ ). The details of this calculation can be found in Appendix [A.14]. The three-point function becomes

$$
\left\langle h^{3}\left(\tau_{e}\right)\right\rangle=(2 \pi)^{3} \delta\left(\mathbf{k}_{1}+\mathbf{k}_{2}+\mathbf{k}_{3}\right) \mathcal{T}_{\text {tmnrpv }}^{(\mathrm{GR})}\left(2 \operatorname{Re}\left[\mathcal{I}^{(\mathrm{GR})}(\tau)\right]\right),
$$

with the polarization expressed in terms of the relations in (2.3.12), as

$$
\begin{aligned}
\mathcal{T}_{\text {tmnrpv }}^{(\mathrm{GR})} & =\left\{\left(\mathbf{k}_{3 l} \cdot \mathbf{k}_{3 k}\right)\left[\Pi_{t m, j l}\left(\mathbf{k}_{1}\right) \Pi_{n r, i k}\left(\mathbf{k}_{2}\right)-\frac{1}{2} \Pi_{t m, k l}\left(\mathbf{k}_{1}\right) \Pi_{n r, i j}\left(\mathbf{k}_{2}\right)\right]\right. \\
& \left.\times \Pi_{p v, i j}\left(\mathbf{k}_{3}\right)+5 \text { perms of } 1,2,3\right\}
\end{aligned}
$$

and where the final result is given in terms of the power spectrum in the nonattractor era (A.14.12), as

$$
2 \operatorname{Re}\left[\mathcal{I}^{(\mathrm{GR})}\right]=-\frac{(2 \pi)^{4} P_{h}^{2}}{\prod_{i} k_{i}^{3}} \frac{K^{3}}{64}\left(1-\frac{3 \sum_{i \neq j} k_{i}^{2} k_{j}}{K^{3}}-\frac{6 \prod_{i} k_{i}}{K^{3}}\right) y_{\text {end }}^{2}+\cdots,
$$

where $K=k_{1}+k_{2}+k_{3}$ and dots indicate terms higher-order in $K y_{\text {end }}$ i.e. $\sim\left(K y_{\text {end }}\right)^{3}$. Here we use "end" to denote the end of the non-attractor phase. In the above, we expressed the result in (6.4.5) in terms of the variable $y$ in (5.3.6). With these ingredients we rewrite the bispectra associated with the "GR" term as

$$
\left\langle h^{3}\left(\tau_{e}\right)\right\rangle=(2 \pi)^{7} \delta\left(\mathbf{k}_{1}+\mathbf{k}_{2}+\mathbf{k}_{3}\right) \frac{P_{h}^{\mathrm{end}}}{\prod k_{i}^{3}} \mathcal{A}_{\text {tmnrpv }}^{\mathrm{GR}},
$$

where we have defined $\mathcal{A}_{\text {tmnrpv }}^{\mathrm{GR}}=\mathcal{A}^{G R}\left(k_{1}, k_{2}, k_{3}\right) \mathcal{T}_{\text {tmnrpv }}^{(\mathrm{GR})}$, with $\mathcal{A}^{\mathrm{GR}}\left(k_{1}, k_{2}, k_{3}\right)=$ $2 \operatorname{Re}\left[\mathcal{I}^{(\mathrm{GR})}\right]$. Next, we consider the second term in (6.4.1),

$$
\mathcal{L}=\frac{a X G_{5 X}}{12} \frac{\phi^{\prime}}{a} h_{i j}^{\prime} h_{j k}^{\prime} h_{k i}^{\prime}=\frac{1}{12} \frac{\sigma_{i} A_{1}^{2} H_{0}}{18 \sqrt{2}} a^{-5} h_{i j}^{\prime} h_{j k}^{\prime} h_{k i}^{\prime}
$$


where

$$
\begin{aligned}
& \frac{\phi^{\prime}}{a}=-\frac{H_{0}^{2} A_{1}}{3} a^{-3}, \quad X=\frac{1}{2}\left(\frac{\phi^{\prime}}{a}\right)^{2}=\frac{1}{2} \frac{H_{0}^{4} A_{1}^{2}}{9} a^{-6}, \\
& G_{5 X}=\frac{\sigma_{i}}{\sqrt{2} H_{0}^{3}} \frac{a}{\phi^{\prime}}=\frac{\sigma_{i}}{\sqrt{2} H_{0}^{3}}\left(-\frac{3}{H_{0}^{2} A_{1}}\right) a^{3} .
\end{aligned}
$$

The three-point function becomes

$$
\begin{aligned}
\left\langle h^{3}\left(\tau_{e}\right)\right\rangle & =2 \operatorname{Re}\left\{-i h\left(k_{1}, \tau_{e}\right) h\left(k_{2}, \tau_{e}\right) h\left(k_{3}, \tau_{e}\right) \int_{-\infty(1-i \epsilon)}^{\tau_{e}} \mathrm{~d} \tau\left(-\frac{1}{2} \frac{\sigma_{1} A_{1}^{2} H_{0}}{18 \sqrt{2}}\right) a^{-5}\right. \\
& \partial_{\tau} h_{s}^{*}\left(k_{1}, \tau\right) \partial_{\tau} h_{s}^{*}\left(k_{2}, \tau\right) \partial_{\tau} h_{s}^{*}\left(k_{3}, \tau\right) \Pi_{t m, i j}\left(\mathbf{k}_{1}\right) \Pi_{n r, j k}\left(\mathbf{k}_{2}\right) \Pi_{p v, k i}\left(\mathbf{k}_{3}\right) \\
& \left.\times(2 \pi)^{3} \delta\left(\mathbf{k}_{1}+\mathbf{k}_{2}+\mathbf{k}_{3}\right)\right\}
\end{aligned}
$$

where the minus sign inside the brackets in the integral comes from taking $\mathcal{L}=-\mathcal{H}_{\text {int }}$ and we have accounted for a symmetry factor of 6 . Again, we express the three-point function as in (6.4.3). We have

$$
\left\langle h^{3}\left(\tau_{e}\right)\right\rangle=(2 \pi)^{3} \delta\left(\mathbf{k}_{1}+\mathbf{k}_{2}+\mathbf{k}_{3}\right) \mathcal{T}_{\text {tmnrpv }}^{\text {(new) }}\left(2 \operatorname{Re}\left[\mathcal{I}^{\text {(new) }}(\tau)\right]\right),
$$

where the polarizations are given by

$$
\mathcal{T}_{\text {tmnrpv }}^{\text {(new) }}=\Pi_{t m, i j}\left(\mathbf{k}_{1}\right) \Pi_{n r, j k}\left(\mathbf{k}_{2}\right) \Pi_{p v, k i}\left(\mathbf{k}_{3}\right),
$$

and where we have used the form of the solutions in (A.14.7), (A.14.8), (A.14.9), and that $a^{-5}=-\left(H_{0} \tau\right)^{5}$, to express

$$
\begin{aligned}
\mathcal{I}^{\text {(new) }} & =-i \frac{y_{0}^{12}}{2^{3}\left(k_{1} k_{2} k_{3}\right)^{3} z_{0}^{6} c_{T}^{18}} \frac{1}{2} \frac{\sigma_{1} A_{1}^{2} H_{0}^{6}}{18 \sqrt{2}} \\
& \times\left(i-c_{T} k_{1} \tau_{e}\right)\left(i-c_{T} k_{2} \tau_{e}\right)\left(i-c_{T} k_{3} \tau_{e}\right) \tau_{e}^{-9} e^{-i c_{T} K \tau_{e}} \\
& \times \int_{-\infty(1-i \epsilon)}^{\tau_{0}} \mathrm{~d} \tau \tau^{-7}\left(3 i+3 c_{T} k_{1} \tau-i c_{T}^{2} k_{1}^{2} \tau^{2}\right)\left(3 i+3 c_{T} k_{2} \tau\right. \\
& \left.-i c_{T}^{2} k_{2}^{2} \tau^{2}\right)\left(3 i+3 c_{T} k_{3} \tau-i c_{T}^{2} k_{3}^{2} \tau^{2}\right) e^{i c_{T} K \tau}
\end{aligned}
$$

where the minus sign from the evaluation of the scale factor cancelled the minus sign from the interaction Hamiltonian and therefore, we have an overall minus sign. The integrals are evaluated by following the same procedure as before. We find that to leading-order this gives

$$
\begin{aligned}
\operatorname{Re}\left[\mathcal{I}^{\text {(new) }}\right] & =\frac{y_{0}^{12}}{2^{3}\left(k_{1} k_{2} k_{3}\right)^{3} z_{0}^{6} c_{T}^{18}} \frac{1}{2} \frac{\sigma_{1} A_{1}^{2} H_{0}^{6}}{18 \sqrt{2}} \\
& \times \tau_{e}^{-12} \frac{3 c_{T}^{3}}{2} K^{3}\left(-1+\frac{3 \sum_{i \neq j} k_{i}^{2} k_{j}}{K^{3}}+\frac{6 \prod_{i} k_{i}}{K^{3}}\right) .
\end{aligned}
$$


The coefficient is evaluated to be

$$
\frac{y_{0}^{12}}{2^{3} z_{0}^{6} c_{T}^{18}} \frac{1}{2} \frac{\sigma_{i} A_{1}^{2} H_{0}^{6}}{18 \sqrt{2}} \frac{3 c_{T}^{3}}{2}=\frac{1}{2} \frac{243 \sqrt{2} \sigma_{1}}{A_{1}^{4} f_{t}^{3} H_{0}^{12}}=\frac{6 \cdot 81 \sigma_{1}}{2 \sqrt{2} A_{1}^{4} f_{t}^{3} H_{0}^{12}}
$$

We finally have

$$
\begin{aligned}
\operatorname{Re}\left[\mathcal{I}^{(\text {new })}\right] & =\frac{6 \cdot 81 \sigma_{1}}{2 \sqrt{2} A_{1}^{4} f_{t}^{3} H_{0}^{12}}\left(\prod_{i} k_{i}\right)^{-3} \\
& \times \tau_{e}^{-12} K^{3}\left(-1+\frac{3 \sum_{i \neq j} k_{i}^{2} k_{j}}{K^{3}}+\frac{6 \prod_{i} k_{i}}{K^{3}}\right) .
\end{aligned}
$$

This can be written in terms of the power spectrum, per polarization, in the nonattractor era (A.14.12), as

$$
\begin{aligned}
2 \operatorname{Re}\left[\mathcal{I}^{(\text {new })}\right] & =\frac{3}{16} \frac{\sigma_{i}}{2 \sqrt{2}} \frac{1}{g_{t}} P_{h}^{2}(2 \pi)^{4}\left(\prod_{i} k_{i}\right)^{-3} \\
& \times K^{3}\left(-1+\frac{3 \sum_{i \neq j} k_{i}^{2} k_{j}}{K^{3}}+\frac{6 \prod_{i} k_{i}}{K^{3}}\right)+\cdots
\end{aligned}
$$

where dots indicate terms higher order in $K y_{\text {end }}$ i.e. $\sim\left(K y_{\text {end }}\right)^{2}$. We can put this into a more condensed form, by defining

$$
\dot{\phi}=\dot{\phi}^{(0)} a^{-3}, \quad X=X^{(0)} a^{-6}, \quad G_{5 X}=G_{5 X}^{(0)} a^{3},
$$

where

$$
\begin{aligned}
\dot{\phi}^{(0)} & =-\frac{H_{0}^{2} \alpha_{1}}{3\left(-\rho_{1}+\delta_{1}+\beta_{1}+\sigma_{1}\right)}, \quad X^{(0)}=\frac{1}{2}\left(\dot{\phi}^{(0)}\right)^{2} \text { and } \\
G_{5 X}^{(0)} & =\frac{\sigma_{1}}{\sqrt{2} H_{0}^{3}}\left(\dot{\phi}^{(0)}\right)^{-1} .
\end{aligned}
$$

Let us also denote the constant

$$
\mu^{(0)}=\dot{\phi}^{(0)} X^{(0)} G_{5 X}^{(0)}
$$

From this we find that the result in (6.4.16) becomes 


$$
\begin{aligned}
2 \operatorname{Re}\left[\mathcal{I}^{(\text {new })}\right] & =\frac{(2 \pi)^{4} P_{h}^{(\text {end })^{2}}}{\prod_{i} k_{i}^{3}} \frac{3 H \mu^{(0)}}{16 \mathcal{G}_{T}^{(0)}} \\
& \times\left\{K^{3}\left(-1+\frac{3 \sum_{i \neq j} k_{i}^{2} k_{j}}{K^{3}}+\frac{6 \prod_{i} k_{i}}{K^{3}}\right)\right. \\
& \left.-\frac{1}{4}\left(K^{3}-5 \sum_{i \neq j} k_{i}^{2} k_{j}+\frac{2}{K^{2}} \sum_{i \neq j} k_{i}^{3} k_{j}^{2}\right)\left(K y_{\text {end }}\right)^{2}\right\} .
\end{aligned}
$$

The contribution of this term to the three-point function can be written similarly to the expression in (6.4.6). In a similar manner, we define the amplitude $\mathcal{A}_{\text {tmnrpv }}^{\text {new }}=$ $\mathcal{A}^{\text {new }}\left(k_{1}, k_{2}, k_{3}\right) \mathcal{T}_{\text {tmnrpv }}^{\text {(new) }}$, with $\mathcal{A}^{\text {new }}\left(k_{1}, k_{2}, k_{3}\right)=2 \operatorname{Re}\left[\mathcal{I}^{\text {(new) }}\right]$.

At leading-order we find that the result is scale-independent. The difference between the scale dependence of the $\mathcal{A}^{\text {GR }}$ and $\mathcal{A}^{\text {new }}$ can be understood by analyzing the time dependence of each term in the interaction Lagrangian (6.4.1). Using that $\mathcal{F}_{T}$ scales like $a^{-6}$ during the non-attractor phase, the "GR" term can be written schematically as $a^{-8} h h h$, while the "new" term scales as $a^{-6} h h h$. From this it is evident why the contribution from each term differs by a factor $\tau^{-2}$ at leading order in the amplitude of the bispectrum.

Before proceeding we should mention that our expressions for the tensor bispectrum contain a characteristic scale dependence $\sim\left(K y_{\text {end }}\right)$ that is distinctive to our scenario - being absent in other frameworks with large tensor non-Gaussianity. We have computed the bispectrum at the end of the non-attractor phase, for which reason there is an explicit dependence in the time $y_{\text {end }}$ in our results. Here, for simplicity, we assume that this era is immediately followed by a slow-roll phase where tensor fluctuations freeze at super-horizon scales. The overall scale dependence of the tensor bispectrum, controlled by $K$, is also distinctive in our setup. This is interesting because a scale-dependent non-Gaussianity might be different at different interferometer scales i.e. LIGO-VIRGO and LISA. It would be interesting to further explore the phenomenological consequences of this property, which goes beyond the scope of our work.

Next, we discuss the physical consequences of the tensor three-point function during a non-attractor era. In order to express our results more concisely it is useful to decompose the transverse-traceless tensor $h_{i j}$ into the helicity basis

$$
\xi^{s}(\mathbf{k}) \equiv h_{i j}(\mathbf{k}) e_{i j}^{* s}(\mathbf{k})
$$

This is because it is more convenient to work with the two independent scalar degrees of freedom in $h_{i j}$ where the right- and left-handed circular polarizations are given by the scalar field operators $\xi^{R} \equiv h_{i j} e_{i j}^{*(+)}$ and $\xi^{L} \equiv h_{i j} e_{i j}^{*(-)}$, respectively $[137,203,253]$. This allows us to express the three-point function in the non-attractor era as 


$$
\begin{aligned}
\left\langle\xi^{\left(s_{1}\right)}\left(\mathbf{k}_{1}\right) \xi^{\left(s_{2}\right)}\left(\mathbf{k}_{2}\right) \xi^{\left(s_{3}\right)}\left(\mathbf{k}_{3}\right)\right\rangle & =(2 \pi)^{7} \delta\left(\mathbf{k}_{1}+\mathbf{k}_{2}+\mathbf{k}_{1}\right) \frac{P_{h}^{(\mathrm{end})^{2}}}{\prod_{i} k_{i}^{3}}\left(\mathcal{A}_{(\mathrm{new})}^{s_{1} s_{2} s_{3}}+\mathcal{A}_{(\mathrm{GR})}^{s_{1} s_{2} s_{3}}\right) \\
& =(2 \pi)^{7} \delta\left(\mathbf{k}_{1}+\mathbf{k}_{2}+\mathbf{k}_{1}\right) B^{s_{1} s_{2} s_{3}}\left(\mathbf{k}_{i}\right)
\end{aligned}
$$

where the bispectrum $B^{s_{1} s_{2} s_{3}}\left(\mathbf{k}_{i}\right)$ is defined as the coefficient of the $\delta$-function and it depends both on the momenta as well the polarization indices. Using (6.4.4) and (6.4.11), the amplitudes

$$
\mathcal{A}_{(\text {new })(\mathrm{GR})}^{s_{1} s_{2} s_{3}}=e_{t m}^{*\left(s_{1}\right)}\left(\mathbf{k}_{1}\right) e_{n r}^{*\left(s_{2}\right)}\left(\mathbf{k}_{2}\right) e_{p v}^{*\left(s_{3}\right)}\left(\mathbf{k}_{3}\right) \mathcal{A}_{\text {tmnrpv }}^{(\text {new })(\mathrm{GR})}
$$

can be calculated following the method in [254,255].

In terms of the helicity notation used here, we are, essentially, calculating the following three-point function $\left\langle\xi^{(R, L)}\left(y_{\text {end }}, \mathbf{k}_{1}\right) \xi^{(R, L)}\left(y_{\text {end }}, \mathbf{k}_{2}\right) \xi^{(R, L)}\left(y_{\text {end }}, \mathbf{k}_{3}\right)\right\rangle$, while on the RHS we will have, schematically, the product $e_{t m}^{* \pm}\left(\mathbf{k}_{1}\right) e_{n r}^{* \pm}\left(\mathbf{k}_{2}\right) e_{p v}^{* \pm}\left(\mathbf{k}_{3}\right)$ contracted with the expressions in (A.14.14) for the "GR" term and in (A.14.15) for the "new", but with the tensor perturbations decomposed in terms of left and right helicity modes (see [256] for more details). Here we do not perform the explicit calculation as in [256], but instead we make direct use of the results in [254,255]. This results in the following two expressions for the scalar amplitudes

$$
\begin{aligned}
& \mathcal{A}_{\text {(new) }}^{s_{1} s_{2} s_{3}}=\mathcal{A}_{(\text {new })}\left(k_{1}, k_{2}, k_{3}\right) F\left(s_{1} k_{1}, s_{2} k_{2}, s_{3} k_{3}\right), \\
& \mathcal{A}_{(\mathrm{GR})}^{s_{1} s_{2} s_{3}}=\mathcal{A}_{(\mathrm{GR})}\left(k_{1}, k_{2}, k_{3}\right) \frac{\left(s_{1} k_{1}+s_{2} k_{2}+s_{3} k_{3}\right)^{2}}{2} F\left(s_{1} k_{1}, s_{2} k_{2}, s_{3} k_{3}\right),
\end{aligned}
$$

where

$$
\begin{aligned}
F\left(s_{1} k_{1}, s_{2} k_{2}, s_{3} k_{3}\right) & =e_{i j}^{*+}\left(\mathbf{k}_{1}\right) e_{j k}^{*+}\left(\mathbf{k}_{2}\right) e_{k i}^{*+}\left(\mathbf{k}_{3}\right)=e_{i j}^{*-}\left(\mathbf{k}_{1}\right) e_{j k}^{*-}\left(\mathbf{k}_{2}\right) e_{k i}^{*-}\left(\mathbf{k}_{3}\right) \\
& =-\frac{\left(k_{1}+k_{2}+k_{3}\right)^{3}\left(k_{1}+k_{2}-k_{3}\right)\left(k_{2}+k_{3}-k_{1}\right)\left(k_{3}+k_{1}-k_{2}\right)}{64\left(k_{1} k_{2} k_{3}\right)^{2}}
\end{aligned}
$$

The derivation of this expression can be found in [256] (Appendix B) and [257] (Appendix C). The amplitudes $\mathcal{A}^{(G R)}$ and $\mathcal{A}^{(\text {new })}$ were given in (6.4.5) (6.4.20), respectively.

Using these results, we can compute the bispectrum in the limit of squeezed isosceles triangle, to find that for $s_{1}=s_{2}$

$$
B^{s_{1} s_{2} s_{3}}\left(\mathbf{k}_{1},-\mathbf{k}_{\mathbf{1}}, \mathbf{k}_{3} \rightarrow 0\right)=\frac{\left(P_{h}^{(\mathrm{end})}\right)^{2}}{32 k_{1}^{3} k_{3}^{3}}\left[\frac{3 H \mu^{(0)}}{\mathcal{G}^{(0)}}\left(1+\frac{\left(k_{1} y_{\mathrm{end}}\right)^{2}}{2}\right)+\frac{\left(k_{1} y_{\mathrm{end}}\right)^{2}}{2}\right]
$$


while for $s_{1} \neq s_{2}$ we find zero. With our notation, Maldacena's consistency condition reads, in this case, as

$$
B^{s_{1} s_{2} s_{3}}\left(\mathbf{k}_{1},-\mathbf{k}_{\mathbf{1}}, \mathbf{k}_{3} \rightarrow 0\right)=\frac{\left(3 P_{h}^{(\text {end })}\right)^{2}}{64 k_{1}^{3} k_{3}^{3}} .
$$

Therefore, we find that Maldacena's consistency relation is violated as during the non-attractor era, besides the usual growing tensor mode, the would-be decaying mode is excited as well and we are no longer working in a single-tensor adiabatic system where Maldacena's arguments apply. This implies that, by tuning the parameters of this model, the amplitudes of the tensor bispectrum can be enhanced in the squeezed limit with potentially interesting phenomenological consequences. Similar considerations have been developed in the scalar sector, see e.g. [159, 258-261], where they found non-attractor models with an enhanced scalar bispectrum in the squeezed limit. See also $[207,208,251,262,263]$ for different scenarios with enhanced tensor bispectrum.

We introduce the following definition of the non-linearity parameter $f_{N L}$, as in $[254,255]$ which is analogous to the standard $f_{N L}$ for the curvature perturbation and it is defined in terms of equilateral configurations for tensor bispectra, as well its value depends on the polarizations

$$
\left.f_{N L(\text { new })(\mathrm{GR})}^{s_{1} s_{2} s_{3}} \equiv 30 \frac{\mathcal{A}_{(\text {new })(\mathrm{GR})}^{s_{1} s_{2} s_{3}}}{K^{3}}\right|_{k_{1}=k_{2}=k_{3}} .
$$

Also, in $[254,255]$ it was found that due the dependence of the non-linearity parameter to the polarization (this can be seen from the definitions in (6.4.24)) one has the following symmetry $f_{N L(\text { new })(\mathrm{GR})}^{++-}=f_{N L(\text { new })(\mathrm{GR})}^{+--}$and $f_{N L(\text { new })(\mathrm{GR})}^{+++}=f_{N L(\text { new })(\mathrm{GR})}^{---}$ Using the definitions for the amplitudes in (6.4.24), we find

$$
f_{N L(\text { new })}^{+++}=\frac{135}{512} \frac{H \mu^{(0)}}{\mathcal{G}^{(0)}}\left(1+\frac{5}{36}\left(K y_{\text {end }}\right)^{2}\right)
$$

and

$$
f_{N L \text { (new) }}^{++-}=\frac{15}{512} \frac{H \mu^{(0)}}{\mathcal{G}^{(0)}}\left(1+\frac{5}{36}\left(K y_{\text {end }}\right)^{2}\right)
$$

Similarly, for the "GR" term we have

$$
f_{N L(\mathrm{GR})}^{+++}=\frac{45}{4096}\left(K y_{\mathrm{end}}\right)^{2} \quad \text { and } \quad f_{N L(\mathrm{GR})}^{++-}=\frac{5}{36864}\left(K y_{\mathrm{end}}\right)^{2} .
$$

From this we conclude that the $f_{N L}$ parameter is positive during the non-attractor phase, which is similar to the contracting Universe considered in [228]. Additionally, due to the strong scale-dependence of $f_{N L(\mathrm{GR})}$ the bispectrum is dominated by 

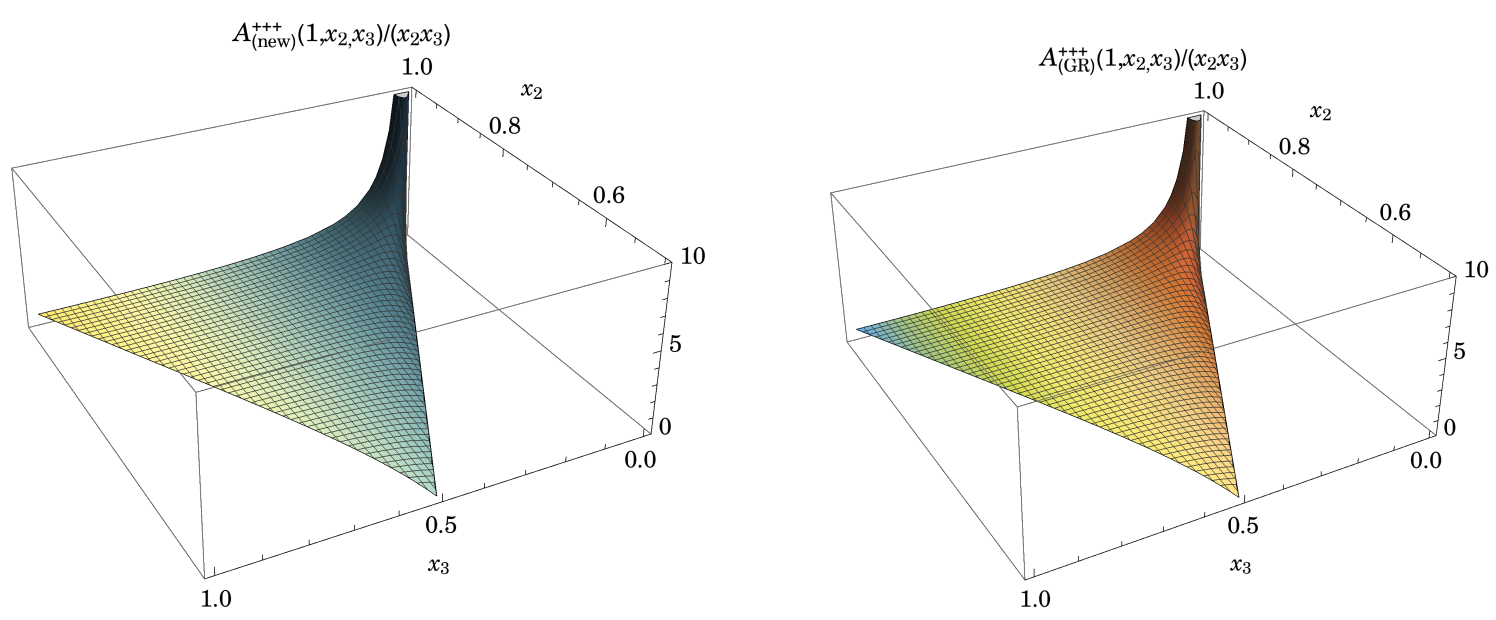

Figure 6.2: $\mathcal{A}_{\text {(new) }}^{+++}\left(1, x_{2}, x_{3}\right) /\left(x_{2} x_{3}\right)$ as a function of $x_{2}$ and $x_{3}$ where we set $H \mu^{\text {end }} / \mathcal{G}_{T}^{\text {end }} \rightarrow 1$ (Left). $\mathcal{A}_{(\mathrm{GR})}^{+++}\left(1, x_{2}, x_{3}\right) /\left(x_{2} x_{3}\right)$ as a function of $x_{2}$ and $x_{3}$ (Right). In both plots we normalized the amplitudes $\mathcal{A}_{(\text {new })}^{+++}$and $\mathcal{A}_{(\mathrm{GR})}^{+++}$to unity for equilateral configurations $x_{2}=x_{3}=1$ and took $-K y_{\mathrm{end}}=10^{-2}$. Image credit: Plots by $O$. Ozsoy.

$f_{N L \text { (new) }}$ for $K y_{\text {end }} \ll 1$ and $H \mu^{(0)} / \mathcal{G}_{T}^{(0)} \sim \mathcal{O}(1)$, in terms of the background Horndeski model we are considering here $\mathcal{G}_{T}=2\left(G_{4}-2 X G_{4 X}-\mu H\right)$. This means that in order to achieve $H \mu^{(0)} / \mathcal{G}_{T}^{(0)} \gg 1$ we need accidental cancellations between the first two terms in $\mathcal{G}_{T}>0$ and $\mu H$. Note that this situation is not special to the model considered here as it can arise for general slow-roll scenarios [43].

In Figure (6.2) we plot the shapes of tensor non-Gaussianity in our model. Since both of the amplitudes have non-trivial scale dependence, we examine the shape of the amplitude for fixed $K y_{\text {end }}$. Here we focus on the dimensionless ratio $\mathcal{A}_{\text {(new)(GR) }}^{s_{1} s_{2} s_{3}} /\left(k_{1} k_{2} k_{3}\right)$ of both amplitudes in (6.4.24) following the literature for scalar perturbations [264]. We see that both the interaction terms in the cubic action give rise to non-Gaussianity that peaks in the squeezed limit. This is due to the fact that during the non-attractor era tensor fluctuations keep growing outside the horizon due to the dynamics of the would-be decaying mode. In standard slow-roll inflation tensor fluctuations freeze at large scales and therefore only wavenumbers comparable to the size of the horizon can contribute to the non-Gaussianity for the "new" term. It was found in $[254,255]$ that this peaks in the equilateral configuration.

\subsection{Disformal transformations and tensor non-Gaussianity}

The general quadratic action for tensors in (5.3.1) can be transformed into a form identical to the action for tensor fluctuations in General Relativity by applying a disformal + conformal transformation to the metric $[135,136]$. In this Appendix, we 
discuss the implications ${ }^{1}$ of such transformations for the background dynamics and for the tensor bispectrum discussed in this Chapter. For our system, the corresponding combination of disformal and conformal transformation, in terms of redefinitions of the time-coordinate and the scale factor, were given in (A.13.6), namely

$$
\mathrm{d} \hat{t}=\left(c_{T} \mathcal{F}_{T}\right)^{1 / 2} \mathrm{~d} t, \quad \hat{a}(\hat{t})=\left(\frac{\mathcal{F}_{T}}{c_{T}}\right)^{1 / 2} a(t) .
$$

Using these, we found that the quadratic action in (5.3.1) takes the standard form of canonical Einstein gravity, as it appears in GR, given by

$$
\begin{aligned}
S_{T}^{(2)} & =\frac{1}{8} \int \mathrm{d} \hat{t} \mathrm{~d}^{3} x \hat{a}(\hat{t})^{3}\left[\left(\partial_{\hat{t}} h_{i j}\right)^{2}-\frac{1}{\hat{a}(\hat{t})^{2}}\left(\nabla h_{i j}\right)^{2}\right] \\
& =\frac{1}{2} \int \mathrm{d} y \mathrm{~d}^{3} x \frac{\hat{a}^{2}}{4}\left[\left(\partial_{y} h_{i j}\right)^{2}-\left(\nabla h_{i j}\right)^{2}\right],
\end{aligned}
$$

where in the second line we have used the fact that the conformal time in the GR frame is defined by the coordinate $y$, namely $\mathrm{d} \hat{t} / \hat{a}(\hat{t}) \equiv \mathrm{d} y$, which can be seen by combining the expressions given in (6.5.1).

In order to describe the time evolution of the background in the Einstein frame, we make use of the relation between two scale factors in (A.13.6) together with the fact that $\mathcal{F}_{T} \propto a^{-6}$ and $a \propto 1 / y$, i.e. $a \propto \mathcal{F}_{T}^{-\frac{1}{2}} \hat{a} \Rightarrow \hat{a} \propto a^{-2}$. This procedure leads to the conclusion that, in the Einstein frame, the Universe appears to be collapsing as in a dust dominated Universe, that is

$$
\hat{a} \propto y^{2}
$$

as $y \rightarrow 0$. Similarly, we can relate the Hubble rate in the Einstein frame, $\hat{H}=$ $\mathrm{d} \ln \hat{a} / \mathrm{d} \hat{t}$, to the Hubble rate in the Jordan frame using (A.13.6), which leads to

$$
\hat{H}=-\frac{2 H}{\left(c_{T} \mathcal{F}_{T}\right)^{\frac{1}{2}}} \propto \hat{a}^{-\frac{3}{2}}
$$

as expected from a dust dominated universe. Using the transformation (6.5.4) for the Hubble rate, the power spectrum of tensor fluctuations in the Jordan frame can be expressed in terms of the quantities in the Einstein frame, as

$$
P_{h}=\frac{\hat{H}^{2}}{2 \pi^{2}} \propto \hat{a}^{-3}
$$

\footnotetext{
${ }^{1}$ See also $[133,265]$ for a general analysis of the consequences of disformal transformations on cosmological fluctuations.
} 
This expression reflects the equivalence of the interpretation of the results in both frames. In the Einstein frame, the power spectrum of tensor fluctuations also appears to be increasing during the transient collapsing ${ }^{2}$ phase as $\hat{a} \rightarrow 0$.

The equivalence of the results in both frames also extends to the observables such as the tensor non-Gaussianity. In the following, we prove that the calculation of the bispectrum is equivalent in both frames. For this purpose, we first realize from (6.5.2) and (6.5.3) that the canonical variable $v_{i j}=z_{T} h_{i j}$ with $z_{T}=\hat{a} / 2$ satisfies the same equation in Fourier space (i.e. see (6.3.1)) similar to the case during the nonattractor phase. Therefore, in the Einstein frame, the mode functions that reduce to the Bunch-Davies vacuum, are given by

$$
h(\mathbf{k}, y)=\frac{-i \hat{H}}{\sqrt{2 k^{3}}}(1+i k y) e^{-i k y} .
$$

Notice that using the relation (6.5.4), the mode functions appear to have the same form as the one in the non-attractor phase, namely

$$
h(\mathbf{k}, y)=\frac{i \sqrt{2} H}{\sqrt{c_{T} \mathcal{F}_{T} k^{3}}}(1+i k y) e^{-i k y},
$$

where $\mathcal{F}_{T}=\mathcal{F}_{T}^{(\text {end })}\left(y / y_{\text {end }}\right)^{6}$. In order to establish the equivalence of the in-in calculation in both frames we therefore, only need to focus on the time-dependence of the interaction Hamiltonian in the Einstein frame, which is given by

$$
H_{\text {int }}(y)=-\int \mathrm{d}^{3} x\left[\frac{Q_{\text {new }}(y)}{12} h_{i j}^{\prime} h_{j k}^{\prime} h_{k i}^{\prime}+\frac{\hat{a}^{2}(y)}{4}\left(h_{i k} h_{j l}-\frac{1}{2} h_{i j} h_{k l}\right) \partial_{k} \partial_{l} h_{i j}\right],
$$

where prime denotes a time derivative with respect to $y$ and we have defined the time dependent pre-factor of the new interaction as

$$
Q_{\text {new }}=\frac{\hat{a} \mathcal{F}_{T}^{3 / 4}}{\mathcal{G}_{T}^{5 / 4}} X \dot{\phi} G_{5 X}
$$

We proceed to the in-in calculation in the Einstein frame by defining analogues of the functions $\mathcal{I}^{\text {(new) }}$ and $\mathcal{I}^{(\mathrm{GR})}$, that we defined earlier in the Jordan frame. Following the same steps as we before, these functions in the Einstein frame, are given by

$$
\hat{\mathcal{I}}^{(\mathrm{GR})}=h\left(\mathbf{k}_{1}, y_{\text {end }}\right) h\left(\mathbf{k}_{2}, y_{\text {end }}\right) h\left(\mathbf{k}_{3}, y_{\text {end }}\right) \int_{-\infty}^{y_{\text {end }}} \mathrm{d} y \frac{\hat{a}^{2}(y)}{4} h^{*}\left(\mathbf{k}_{1}, y\right) h^{*}\left(\mathbf{k}_{2}, y\right) h^{*}\left(\mathbf{k}_{3}, y\right)
$$

\footnotetext{
${ }^{2}$ Note that similar to the time span $y_{0}<y<y_{\text {end }}$ of the non-attractor era in the Jordan frame, the collapsing phase in the Einstein frame will last for a finite time.
} 
$\hat{\mathcal{I}}^{(\text {new })}=-h\left(\mathbf{k}_{1}, y_{\text {end }}\right) h\left(\mathbf{k}_{2}, y_{\text {end }}\right) h\left(\mathbf{k}_{3}, y_{\text {end }}\right) \int_{-\infty}^{y_{\text {end }}} \mathrm{d} y \frac{Q_{\text {new }}(y)}{2} h^{*}\left(\mathbf{k}_{1}, y\right) h^{*}\left(\mathbf{k}_{2}, y\right) h^{*}\left(\mathbf{k}_{3}, y\right)$,

where $y_{\text {end }}$ denotes the end of the collapsing phase. Noting $\hat{a} \propto y^{2}$ and $Q_{\text {new }} \propto \hat{a}^{5 / 2} \propto$ $y^{5}$ and the mode functions in (6.5.7), we see that we need to deal with identical integrals, to the ones we solved earlier in the Jordan frame, in the calculation of the bispectrum amplitude in the Einstein frame.

\subsection{Conclusions}

We investigated the consequences of a non-attractor phase of cosmological evolution for the dynamics of primordial tensor modes, focussing on the properties of primordial tensor non-Gaussianity in scenarios with non-minimal couplings of gravity to the scalar sector. Thanks to a tensor duality, we have been able to analytically compute the properties of the tensor bispectrum during this phase. We have shown that the tensor bispectrum is enhanced in the squeezed limit with respect to standard slow-roll scenarios and can parametrically violate Maldacena's consistency relations. Moreover, tensor non-Gaussianity exhibits a scale dependence characteristic of our set-up, that can help to distinguish our model from other scenarios with large tensor non-Gaussianity.

Much work is left for the future. It would be interesting to apply our approach to more general scenarios then the ones considered so far, including theories of beyond Horndeski or DHOST [130,131,266-268]. This would also allow one to study in more general terms the transition phase between attractor and non-attractor and related possible instabilities associated with violations of energy conditions (see the discussion in the Appendix of [8]). We plan to return on these topics soon. 


\section{A. Appendix}

\section{A.1 Covariant energy-momentum conservation law}

The generalized stress-tensor describes the coupling of matter and radiation to the gravitational field through the Einstein's field equations [269]. The variation of the action for the matter fields $S_{M}$ with respect to variations in the metric $g_{\mu \nu}(x)$ leads to the covariant energy-momentum conservation law. We start with an infinitesimal coordinate transformation of spacetime $x^{\mu} \rightarrow x^{\prime \mu}=x^{\mu}-\epsilon^{\mu}(x)$ and take $g_{\mu \nu}(x)$ to transform as

$$
g_{\mu \nu}(x) \rightarrow g_{\mu \nu}^{\prime}\left(x^{\prime}\right)=\frac{\partial x^{\lambda}}{\partial x^{\prime \mu}} \frac{\partial x^{\sigma}}{\partial x^{\prime \nu}} g_{\lambda \sigma}(x),
$$

where $x$ and $x^{\prime}$ refer to the same event in spacetime and $\epsilon^{\mu}(x)$ is an infinitesimal quantity. Under this variation the metric becomes, see (Appendix A.3)

$$
\delta g_{\mu \nu}(x) \rightarrow g_{\mu \nu}^{\prime}\left(x^{\prime}\right)-g_{\mu \nu}(x)=\left(\nabla_{\mu} \epsilon_{\nu}+\nabla_{\nu} \epsilon_{\mu}\right) \delta g_{\mu \nu}(x)=\mathcal{L}_{\epsilon} \delta g_{\mu \nu}(x),
$$

where $\mathcal{L}_{\epsilon}=\nabla_{\mu} \epsilon_{\nu}+\nabla_{\nu} \epsilon_{\mu}$ is the Lie derivative. In the following we will drop the index $M$ from the matter action.

Consider variation of the matter action with respect to a variation in the metric. Then, using the formulas above, we have

$$
\begin{aligned}
\delta S & =\int \mathrm{d}^{n} x \frac{\delta S}{\delta g_{\mu \nu}(x)} g_{\mu \nu}(x)=\int \mathrm{d} v_{x}|g|^{-\frac{1}{2}} \frac{\delta S}{\delta g_{\mu \nu}(x)} g_{\mu \nu}(x) \\
& =\int \mathrm{d} v_{x}|g|^{-\frac{1}{2}} \frac{\delta S}{\delta g_{\mu \nu}(x)}\left(\nabla_{\mu} \epsilon_{\nu}+\nabla_{\nu} \epsilon_{\mu}\right)=2 \int \mathrm{d} v_{x}|g|^{-\frac{1}{2}} \frac{\delta S}{\delta g_{\mu \nu}(x)} \nabla_{\mu} \epsilon_{\nu} \\
& =-\int \mathrm{d} v_{x} T^{\mu \nu} \nabla_{\mu} \epsilon_{\nu}=0,
\end{aligned}
$$

where we have used that $\partial_{\mu} \epsilon^{\nu}$ vanish at the boundaries of the region $\mathcal{R}$ and required that $\delta S=0$. Now, using the product rule for covariant differentiation one can write

$$
\nabla_{\mu}\left(T_{\mu \nu} \epsilon_{\nu}\right)=\left(\nabla_{\mu} T^{\mu \nu}\right) \epsilon_{\nu}+T^{\mu \nu}\left(\nabla_{\mu} \epsilon_{\nu}\right) \Leftrightarrow T^{\mu \nu}\left(\nabla_{\mu} \epsilon_{\nu}\right)=\nabla_{\mu}\left(T^{\mu \nu} \epsilon_{\nu}\right)-\left(\nabla_{\mu} T^{\mu \nu}\right) \epsilon_{\nu}
$$

Then, the variation of the action becomes 


$$
\delta S=-\int \mathrm{d} v_{x} \nabla_{\mu}\left(T^{\mu \nu} \epsilon_{\nu}\right)+\int \mathrm{d} v_{x}\left(\nabla_{\mu} T^{\mu \nu}\right) \epsilon_{\nu}=\int \mathrm{d} v_{x}\left(\nabla_{\mu} T^{\mu \nu}\right) \epsilon_{\nu}=0
$$

where the surface integral $\int \mathrm{d} v_{x} \nabla_{\mu}\left(T^{\mu \nu} \epsilon_{\nu}\right)$ vanishes, as $\epsilon_{\nu}$ is taken to be zero at the boundary. As $\epsilon_{\nu}(x)$ is just an arbitrary term, then for the last equation to hold, one requires that

$$
\nabla_{\mu} T^{\mu \nu}=0
$$

That is, the generalized energy momentum tensor is conserved, i.e. this is the covariant generalization of $\partial_{\mu} T^{\mu \nu}=0$. Finally, lowering the indices of the generalized EMT, will give

$$
T_{\mu \nu} \equiv \frac{2}{|g|^{\frac{1}{2}}} \frac{\delta S_{M}}{\delta g^{\mu \nu}(x)},
$$

where the variation of the inverse metric relates to $\delta g_{\mu \nu}$ by $\delta g^{\mu \nu}=-g^{\mu \rho} g^{\nu \sigma} \delta g_{\rho \sigma}$ i.e. one cannot use the unperturbed metric to raise and lower indices of the perturbed metric (see Appendix A.2).

\section{A.2 Variation of the inverse metric}

The variation of the inverse metric is most easily achieved by noting that

$$
g^{\mu \rho} g_{\rho \nu}=\delta^{\mu}{ }_{\nu}
$$

and using the fact that the tensor $\delta^{\mu}{ }_{\nu}$ is constant and it does not change under a variation, i.e. $\delta \delta^{\mu}{ }_{\nu}=0$. Using the product rule and performing the variation to first-order, this becomes

$$
\delta\left(g^{\mu \rho} g_{\rho \nu}\right)=\left(\delta g^{\mu \rho}\right) g_{\rho \nu}+g^{\mu \rho}\left(\delta g_{\rho \nu}\right)=0 .
$$

Multiplying by $g^{\nu \sigma}$, relabelling indices and rearranging gives

$$
\begin{aligned}
& \Leftrightarrow g^{\rho \sigma} g_{\rho \nu} \delta g^{\mu \nu}+g^{\mu \rho} g^{\nu \sigma} \delta g_{\rho \nu}=0 \\
& \Leftrightarrow \delta^{\sigma}{ }_{\nu} \delta g^{\mu \nu}=-g^{\mu \rho} g^{\nu \sigma} \delta g_{\rho \nu} \\
& \Leftrightarrow \delta g^{\mu \nu}=-g^{\mu \rho} g^{\nu \sigma} \delta g_{\rho \sigma} .
\end{aligned}
$$

\section{A.3 Coordinate transformation}

Consider a coordinate transformation of spacetime

$$
g_{\mu \nu}(x) \rightarrow g_{\mu \nu}^{\prime}\left(x^{\prime}\right)=\frac{\partial x^{\lambda}}{\partial x^{\prime \mu}} \frac{\partial x^{\sigma}}{\partial x^{\prime \nu}} g_{\lambda \sigma}(x) .
$$


This is solved by using an infinitesimal coordinate transformation, of the form

$$
x^{\mu} \rightarrow x^{\prime \mu}=x^{\mu}-\epsilon^{\mu}(x),
$$

where $x$ and $x^{\prime}$ refer to the same event in spacetime and $\epsilon^{\mu}(x)$ is an infinitesimal quantity. To first-order in powers of $\epsilon$, this becomes

$$
\frac{\partial x^{\lambda}}{\partial x^{\prime \mu}}=\frac{\partial x^{\prime \lambda}}{\partial x^{\prime \mu}}+\frac{\partial \epsilon^{\lambda}}{\partial x^{\prime \mu}}=\delta_{\mu}^{\lambda}+\frac{\partial \epsilon^{\lambda}}{\partial x^{\mu}}+O(\epsilon)^{2}
$$

where

$$
\frac{\partial \epsilon^{\lambda}}{\partial x^{\prime \mu}}=\frac{\partial \epsilon^{\lambda}}{\partial x^{\beta}} \frac{\partial x^{\beta}}{\partial x^{\prime \mu}}=\frac{\partial \epsilon^{\lambda}}{\partial x^{\beta}} \frac{\partial\left(x^{\prime \beta}+\epsilon^{\beta}\right)}{\partial x^{\prime \mu}}=\delta_{\mu}^{\beta} \frac{\partial \epsilon^{\lambda}}{\partial x^{\beta}}+O(\epsilon)^{2}=\frac{\partial \epsilon^{\lambda}}{\partial x^{\mu}}+O(\epsilon)^{2},
$$

and where $\partial_{\nu^{\prime}} x^{\sigma}=\delta^{\sigma}{ }_{\nu}+\partial_{\nu} \epsilon^{\sigma}$. Here we switched to condensed notation where the index carries the prime of the coordinate for convenience. Finally, we Taylor expand $g_{\lambda \sigma}(x+\epsilon)=g_{\lambda \sigma}(x)+\epsilon^{\alpha} \partial_{\alpha} g_{\lambda \sigma}(x)$, which yields the variation

$$
\begin{aligned}
\delta g_{\mu \nu}(x) & =g_{\mu \nu}^{\prime}\left(x^{\prime}\right)-g_{\mu \nu}(x) \\
& =\left(\delta^{\lambda}{ }_{\mu}+\partial_{\mu} \epsilon^{\lambda}\right)\left(\delta^{\sigma}{ }_{\nu}+\partial_{\nu} \epsilon^{\sigma}\right)\left(g_{\lambda \sigma}+\epsilon^{\alpha} \partial_{\alpha} g_{\lambda \sigma}(x)\right)-g_{\mu \nu} \\
& =\left(\delta^{\lambda}{ }_{\mu} \delta^{\sigma}{ }_{\nu}+\delta^{\lambda}{ }_{\mu} \partial_{\nu} \epsilon^{\sigma}+\partial_{\mu} \epsilon^{\lambda} \delta^{\sigma}{ }_{\nu}+\delta^{\lambda}{ }_{\mu} \delta^{\sigma}{ }_{\nu} \epsilon^{\alpha} \partial_{\alpha}\right) g_{\lambda \sigma}-g_{\mu \nu} \\
& =\left(\partial_{\nu} \epsilon^{\sigma}\right) g_{\mu \sigma}+\left(\partial_{\mu} \epsilon^{\lambda}\right) g_{\lambda \nu}+\epsilon^{\alpha}\left(\partial_{\alpha} g_{\mu \nu}\right) \\
& =\partial_{\nu}\left(\epsilon^{\sigma} g_{\mu \sigma}\right)-\epsilon^{\sigma}\left(\partial_{\nu} g_{\mu \sigma}\right)+\partial_{\mu}\left(\epsilon^{\lambda} g_{\lambda \nu}\right)-\epsilon^{\lambda}\left(\partial_{\mu} g_{\lambda \nu}\right)+\epsilon^{\alpha}\left(\partial_{\alpha} g_{\mu \nu}\right),
\end{aligned}
$$

where we have neglected terms of order $\epsilon^{2}$ and in the last step we used the product rule.

Next, we rewrite the contravariant components in terms of covariant components. For example, the first term becomes $\partial_{\nu}\left(\epsilon^{\sigma} g_{\mu \sigma}\right)=\partial_{\nu}\left(\epsilon_{\beta} g_{\mu \sigma} g^{\sigma \beta}\right)=\partial_{\nu}\left(\epsilon_{\beta} \delta^{\beta}{ }_{\mu}\right)=\partial_{\nu} \epsilon_{\mu}$ and similar for the rest terms. Finally, after relabelling indices this becomes

$$
\begin{aligned}
& =\partial_{\nu} \epsilon_{\mu}+\partial_{\mu} \epsilon_{\nu}-\epsilon_{\alpha} g^{\rho \alpha}\left(\partial_{\mu} g_{\rho \nu}+\partial_{\nu} g_{\rho \mu}-\partial_{\rho} g_{\mu \nu}\right) \\
& =\partial_{\nu} \epsilon_{\mu}+\partial_{\mu} \epsilon_{\nu}-2 \epsilon_{\alpha} g^{\rho \alpha} \Gamma_{\rho \mu \nu} \\
& =\partial_{\nu} \epsilon_{\mu}+\partial_{\mu} \epsilon_{\nu}-2 \epsilon_{\alpha} \Gamma^{\alpha}{ }_{\mu \nu} \\
& =\partial_{\mu} \epsilon_{\nu}-\epsilon_{\alpha} \Gamma^{\alpha}{ }_{\mu \nu}+\partial_{\nu} \epsilon_{\mu}-\epsilon_{\alpha} \Gamma_{\nu \mu}^{\alpha} \\
& =\nabla_{\mu} \epsilon_{\nu}+\nabla_{\nu} \epsilon_{\mu} \\
& =\mathcal{L}_{\epsilon} g_{\mu \nu}
\end{aligned}
$$

where the Christoffel symbols were defined earlier in the text. Here, $\mathcal{L}_{\epsilon}$ denotes the Lie derivative.

\section{A.4 Friedmann Equations}

To evaluate the EFE (1.1.25) in terms of the FRW metric (1.2.6) one first needs to produce the following Christoffel symbols. Using (1.1.3) then we find that 


$$
\begin{aligned}
& \Gamma_{r r}^{0}=\frac{a \dot{a}}{1-\mathcal{K} r^{2}}, \quad \Gamma_{\theta \theta}^{0}=a \dot{a} r^{2}, \quad \Gamma_{\phi \phi}^{0}=a \dot{a} \sin ^{2} \theta r^{2}, \quad \Gamma_{\phi \theta}^{\phi}=\frac{1}{\tan \theta}, \\
& \Gamma_{0 r}^{r}=\Gamma_{0 \theta}^{\theta}=\Gamma_{0 \phi}^{\phi}=\frac{\dot{a}}{a}, \quad \Gamma_{r \theta}^{\theta}=\Gamma_{r \phi}^{\phi}=\frac{1}{r}, \quad \Gamma_{\phi \phi}^{\theta}=-\cos \theta \sin \theta, \\
& \Gamma_{r r}^{r}=\frac{\mathcal{K} r}{1-\mathcal{K} r^{2}}, \quad \Gamma_{\theta \theta}^{r}=-r\left(1-\mathcal{K} r^{2}\right), \quad \Gamma_{\phi \phi}^{r}=-r \sin ^{2} \theta\left(1-\mathcal{K} r^{2}\right), \\
& \Gamma_{00}^{0}=\Gamma_{\theta \theta}^{\theta}=\Gamma_{\phi \phi}^{\phi}=\Gamma_{00}^{r}=\Gamma_{r r}^{\theta}=\Gamma_{r r}^{\phi}=\Gamma_{\theta r}^{r}=\Gamma_{\phi r}^{r}=0,
\end{aligned}
$$

where Christoffel symbols containing more than two different indices also vanish, i.e. $\Gamma_{\phi 0}^{r}=0$ and so on. The time-component of the Ricci tensor (1.1.6) is evaluated to be

$$
R_{00}=R_{0 r 0}^{r}+R_{0 \theta 0}^{\theta}+R_{0 \phi 0}^{\phi}=-3 \frac{\ddot{a}}{a},
$$

and the spatial-components become

$$
3 R_{r r}=3\left(R_{r 0 r}^{0}+R_{r \theta r}^{\theta}+R_{r \phi r}^{\phi}\right)=3\left[\frac{a \ddot{a}}{1-\mathcal{K} r^{2}}+2 \frac{(\dot{a})^{2}}{1-\mathcal{K} r^{2}}+2 \frac{\mathcal{K}}{1-\mathcal{K} r^{2}}\right] .
$$

Then the Ricci scalar (1.1.7) is evaluated to be

$$
\begin{aligned}
R & =g^{\beta \delta} R_{\beta \delta}=g^{00} R_{00}+g^{i j} R_{i j}=3 \frac{\ddot{a}}{a}+3\left[\frac{\ddot{a}}{a}+2\left(\frac{\dot{a}}{a}\right)^{2}+2 \frac{\mathcal{K}}{a^{2}}\right] \\
& =6\left[\frac{\ddot{a}}{a}+\left(\frac{\dot{a}}{a}\right)^{2}+\frac{\mathcal{K}}{a^{2}}\right],
\end{aligned}
$$

where we only had to calculate the radial component of the Ricci tensor and mutiply it by 3, as the FRW metric describes a homogeneous and isotropic universe and therefore it does not distinguish between the $r, \theta$, and $\phi$ directions. Here, we are working from the frame where galaxies are at rest therefore, we set $u_{\mu}=(1,0,0,0)$, and the EMT has components $T_{\mu \nu}=\operatorname{diag}\left(\rho, p g_{r r}, p g_{\theta \theta}, p g_{\phi \phi}\right)$ and trace $g^{\mu \nu} T_{\mu \nu}=$ $T=\operatorname{diag}(-\rho, p, p, p)$. The time-component of the EFE becomes

$$
\begin{aligned}
& R_{00}-\frac{1}{2} R g_{00}+\Lambda g_{00}=8 \pi G T_{00} \Rightarrow-3 \frac{\ddot{a}}{a}+\frac{1}{2} 6\left[\frac{\ddot{a}}{a}+\left(\frac{\dot{a}}{a}\right)^{2}+\frac{\mathcal{K}}{a^{2}}\right]-\Lambda=8 \pi G \rho \\
\Rightarrow & \left(\frac{\dot{a}}{a}\right)^{2}+\frac{\mathcal{K}}{a^{2}}-\frac{\Lambda}{3}-\frac{8 \pi G}{3} \rho=0,
\end{aligned}
$$

which is the first Friedmann equation. The spatial-components of the EFE evaluate to (i.e. consider the radial component as the factors of 3 drops at the end of the calculation) 


$$
\begin{aligned}
& R_{r r}-\frac{1}{2} R g_{r r}+\Lambda g_{r r}=8 \pi G T_{r r} \\
\Rightarrow & \frac{g_{r r}}{a^{2}}\left(a \ddot{a}+2(\dot{a})^{2}+2 \mathcal{K}\right)-\frac{1}{2} R g_{r r}+\Lambda g_{r r}=8 \pi G T_{r r} \\
\Rightarrow & -2 \frac{\ddot{a}}{a}-\left(\frac{\dot{a}}{a}\right)^{2}-\frac{\mathcal{K}}{a^{2}}+\Lambda=8 \pi G g^{r r} T_{r r} \\
\Rightarrow & \frac{\ddot{a}}{a}+\frac{1}{2}\left(\frac{\dot{a}}{a}\right)^{2}+\frac{1}{2} \frac{\mathcal{K}}{a^{2}}-\frac{1}{2} \Lambda+4 \pi G p=0,
\end{aligned}
$$

where we expressed the radial component of the metric as $\left(1-\mathcal{K} r^{2}\right)^{-1}=g_{r r} a^{-2}$. This is the second Friedmann equation. Subtracting the second from the first Friedmann equation gets rid of the factor $\left(\dot{a} a^{-1}\right)^{2}$, giving the more familiar form of the Friedmann equation

$$
\frac{\ddot{a}}{a}+4 \pi G\left(p+\frac{\rho}{3}\right)-\frac{\Lambda}{3}=0 .
$$

\section{A.5 Scalar field equations of motion}

Here we consider the Lagrangian density

$$
\mathcal{L}=\sqrt{-g} L=\sqrt{-g}\left[\frac{1}{2} g^{\mu \nu} \partial_{\mu} \phi \partial_{\nu} \phi+U(\phi)\right]
$$

The Euler-Lagrange equations read

$$
\partial^{\mu}\left[\frac{\partial \mathcal{L}}{\partial\left(\partial_{\mu} \phi\right)}\right]=\square \phi-\frac{\partial U(\phi)}{\partial \phi}
$$

where $\square \phi=g^{\mu \nu} \nabla_{\mu} \nabla_{\nu} \phi$. Using that

$$
\nabla_{\mu} \nabla_{\nu} \phi \equiv \frac{1}{\sqrt{-g}} \partial_{\mu}\left(\sqrt{-g} g^{\mu \nu} \partial_{\nu} \phi\right)
$$

for a homogeneous scalar field $\phi=\phi(t)$ in FRW spacetime $\sqrt{-g}=a^{3}$, we find

$$
\ddot{\phi}+3 H \dot{\phi}+\frac{\partial U(\phi)}{\partial \phi}=0
$$

where an overdot indicates a derivative with respect to time $t$ and the Hubble parameter is defined by $H=\dot{a} / a$. 


\section{A.6 Perturbations in de Sitter spacetime}

The leading order term is given by the Einstein-Hilbert action with a cosmological constant, which in de Sitter it is defined as $\Lambda=3 \mathcal{H}^{2} a^{-2}$, where $\mathcal{H}=a H$. Therefore, we have

$$
S_{E H}=\int \mathrm{d}^{4} x \sqrt{-g} \frac{M_{p l}^{2}}{2}\left(R-6 \frac{\mathcal{H}^{2}}{a^{2}}\right) .
$$

Expanding the first term to second-order in perturbations of the metric, over an FRW background, gives

$$
\begin{aligned}
\frac{M_{p l}^{2}}{2} \sqrt{-g} R & =a^{2} \frac{M_{p l}^{2}}{4}\left[-3\left(h_{i j}\right)^{\prime}\left(h^{i j}\right)^{\prime}-4\left(h^{i j}\right)\left(h_{i j}\right)^{\prime \prime}-12 \mathcal{H}\left(h^{i j}\right)\left(h_{i j}\right)^{\prime}\right. \\
& -6 \mathcal{H}^{2}\left(h_{i j}\right)\left(h^{i j}\right)-6 \mathcal{H}^{\prime}\left(h_{i j}\right)\left(h^{i j}\right)+4\left(h^{i j}\right)\left(\partial_{r} \partial^{r} h_{i j}\right)-2\left(\partial_{j} h_{i r}\right)\left(\partial^{r} h^{i j}\right) \\
& \left.+3\left(\partial_{r} h_{i j}\right)\left(\partial^{r} h^{i j}\right)\right]
\end{aligned}
$$

where primes denote differentiation with respect to time. Next, we perform partial integrations and drop the total derivatives. The first and the last term stay as they are. The second term is partially integrated to give contributions that will be added to the first and the third term

$$
-4 a^{2}\left(h^{i j}\right)\left(h_{i j}\right)^{\prime \prime}=4 a^{2}\left(h_{i j}\right)^{\prime}\left(h^{i j}\right)^{\prime}+8 a a^{\prime}\left(h_{i j}\right)^{\prime}\left(h^{i j}\right) .
$$

The sixth term is partially integrated to give contributions to the last term

$$
4 a^{2}\left(h^{i j}\right)\left(\partial_{r} \partial^{r} h_{i j}\right)=-4 a^{2}\left(\partial_{r} h^{i j}\right)\left(\partial^{r} h_{i j}\right)
$$

The seventh term is partially integrated and removed by using TT-gauge i.e. $\partial_{j} h^{i j}=$ 0 . Adding terms gives

$$
\begin{aligned}
\frac{M_{p l}^{2}}{2} \sqrt{-g} R & =\frac{M_{p l}^{2}}{4}\left[a^{2}\left(h_{i j}\right)^{\prime}\left(h^{i j}\right)^{\prime}-a^{2}\left(\partial_{r} h_{i j}\right)\left(\partial^{r} h^{i j}\right)\right. \\
& \left.-4 a^{2} \mathcal{H}\left(h_{i j}\right)^{\prime}\left(h^{i j}\right)-6 a^{2}\left(\mathcal{H}^{2}+\mathcal{H}^{\prime}\right)\left(h_{i j}\right)\left(h^{i j}\right)\right],
\end{aligned}
$$

where we have used that $a a^{\prime}=a^{2} \mathcal{H}$. The third term in the above expression is partially integrated again, to give contributions to the last term 


$$
-4 a^{2} \mathcal{H}\left(h^{i j}\right)\left(h_{i j}\right)^{\prime}=4 a a^{\prime} \mathcal{H}\left(h_{i j}\right)\left(h^{i j}\right)+2 a^{2} \mathcal{H}^{\prime}\left(h_{i j}\right)\left(h^{i j}\right) .
$$

The expression becomes

$$
\begin{aligned}
\frac{M_{p l}^{2}}{2} \sqrt{-g} R & =a^{2} \frac{M_{p l}^{2}}{4}\left[\left(h_{i j}\right)^{\prime}\left(h^{i j}\right)^{\prime}-\left(\partial_{r} h_{i j}\right)\left(\partial^{r} h^{i j}\right)\right. \\
& \left.-2\left(\mathcal{H}^{2}+2 \mathcal{H}^{\prime}\right)\left(h_{i j}\right)\left(h^{i j}\right)\right],
\end{aligned}
$$

where $a a^{\prime} \mathcal{H}=\left(a^{\prime}\right)^{2}=a^{2} \mathcal{H}^{2}$. Next, we expand the second term in (A.6.1) where $\sqrt{-g}=\sqrt{-\bar{g}}\left(1-\frac{1}{4} \delta_{\gamma}^{\gamma} h^{\mu \nu} h_{\mu \nu}\right)=\sqrt{-\bar{g}}\left(1-h^{\mu \nu} h_{\mu \nu}\right)$ to get

$$
\begin{gathered}
-\frac{M_{p l}^{2}}{2} \sqrt{-g} 6 \frac{\mathcal{H}^{2}}{a^{2}}=-a^{2} \frac{M_{p l}^{2}}{4}\left(-h_{i j} h^{i j}\right) 6 \mathcal{H}^{2}=a^{2} \frac{M_{p l}^{2}}{4} 6 \mathcal{H}^{2}\left(h_{i j} h^{i j}\right) \\
S_{E H}^{(2)}=\int \mathrm{d}^{4} x a^{2} \frac{M_{p l}^{2}}{4}\left[\left(h_{i j}\right)^{\prime}\left(h^{i j}\right)^{\prime}-\left(\partial_{r} h_{i j}\right)\left(\partial^{r} h^{i j}\right)\right] .
\end{gathered}
$$

where $\left(-2 \mathcal{H}^{2}+6 \mathcal{H}^{2}-4 \mathcal{H}^{\prime}\right)\left(h_{i j} h^{i j}\right)=0$ in de Sitter, because for $a=-(H \eta)^{-1}$, we get $\mathcal{H}^{2}=\mathcal{H}^{\prime}=\eta^{-2}$. From this we get that the leading order equations of motion are given by

$$
h_{i j}^{\prime \prime}+2 \frac{a^{\prime}}{a} h_{i j}^{\prime}-\partial_{k} \partial^{k} h_{i j}=0
$$

\section{A.7 Weyl tensor squared}

At the background level the Weyl squared tensor is given by

$$
S_{W^{2}}^{(0)}=\frac{M_{P l}^{2}}{2} \int \mathrm{d}^{4} x \sqrt{-g} \frac{f_{9}}{M^{2}} W^{\mu \nu \rho \sigma} W_{\mu \nu \rho \sigma} .
$$

We expand to second-order in metric perturbations, around an FRW background. In conformal time we get,

$$
S_{W^{2}}^{(2)}=\frac{M_{P l}^{2}}{2} \int \mathrm{d}^{4} x a^{4} \frac{f_{9}}{M^{2}}\left\{2\left[\frac{h_{i j}^{\prime \prime}}{a^{2}}+\frac{\nabla^{2}}{a^{2}} h_{i j}\right]^{2}+8\left(h^{i j}\right)^{\prime} \frac{\nabla^{2}}{a^{4}}\left(h_{i j}\right)^{\prime}\right\} .
$$

We expand the square 


$$
\begin{aligned}
S_{W^{2}}^{(2)} & =\frac{M_{P l}^{2}}{2} \int \mathrm{d}^{4} x \frac{f_{9}}{M^{2}}\left\{2\left[\left(h_{i j}^{\prime \prime}\right)^{2}+2 h_{i j}^{\prime \prime} \nabla^{2} h_{i j}+\left(\nabla^{2} h_{i j}\right)^{2}\right]\right. \\
& \left.+8\left(h^{i j}\right)^{\prime} \nabla^{2}\left(h_{i j}\right)^{\prime}\right\} .
\end{aligned}
$$

The leading order equations of motion for tensors, read

$$
h_{i j}^{\prime \prime}+2 \mathcal{H} h_{i j}^{\prime}-\nabla^{2} h_{i j}=0,
$$

where $\mathcal{H}(\tau)=\frac{a^{\prime}(\tau)}{a(\tau)}$ is the Hubble parameter in conformal time. We can use it to reduce the order of time derivatives, as long as the contributions coming from the Weyl squared term are treated perturbatively. We find

$$
\begin{aligned}
S_{W^{2}}^{(2)} & =\frac{M_{P l}^{2}}{2} \int \mathrm{d}^{4} x \frac{f_{9}}{M^{2}}\left\{2 \left[\left(\nabla^{2} h_{i j}-2 \mathcal{H} h_{i j}^{\prime}\right)^{2}+2\left(\nabla^{2} h_{i j}-2 \mathcal{H} h_{i j}^{\prime}\right) \nabla^{2} h_{i j}\right.\right. \\
& \left.\left.+\left(\nabla^{2} h_{i j}\right)^{2}\right]+8\left(h^{i j}\right)^{\prime} \nabla^{2}\left(h_{i j}\right)^{\prime}\right\} .
\end{aligned}
$$

This simplifies to

$$
\begin{aligned}
S_{W^{2}}^{(2)} & =\frac{M_{P l}^{2}}{2} \int \mathrm{d}^{4} x \frac{f_{9}}{M^{2}}\left\{8 \mathcal{H}^{2}\left(h_{i j}^{\prime}\right)^{2}-16 \mathcal{H} h_{i j}^{\prime} \nabla^{2} h_{i j}\right. \\
& \left.+8\left(h^{i j}\right)^{\prime} \nabla^{2}\left(h_{i j}\right)^{\prime}+8\left(\nabla^{2} h_{i j}\right)^{2}\right\} .
\end{aligned}
$$

Integrating the second term by parts, gives

$$
\begin{aligned}
S_{W^{2}}^{(2)} & =\frac{M_{P l}^{2}}{2} \int \mathrm{d}^{4} x\left[\frac{8 f_{9}}{M^{2}} \mathcal{H}^{2}\left(h_{i j}^{\prime}\right)^{2}-8\left(\frac{f_{9}^{\prime}}{M^{2}} \mathcal{H}+\frac{f_{9}}{M^{2}} \mathcal{H}^{\prime}\right)\left(\nabla h_{i j}\right)^{2}\right. \\
& \left.+\frac{8 f_{9}}{M^{2}} h_{i j}^{\prime} \nabla^{2} h_{i j}^{\prime}+\frac{8 f_{9}}{M^{2}}\left(\nabla^{2} h_{i j}\right)^{2}\right],
\end{aligned}
$$

Finally, taking a factor of $1 / 4$ out, this can be written as

$$
\begin{aligned}
S_{W^{2}}^{(2)} & =\frac{M_{P l}^{2}}{8} \int \mathrm{d}^{4} x\left[\frac{32 f_{9}}{M^{2}} \mathcal{H}^{2}\left(h_{i j}^{\prime}\right)^{2}-32\left(\frac{f_{9}^{\prime}}{M^{2}} \mathcal{H}+\frac{f_{9}}{M^{2}} \mathcal{H}^{\prime}\right)\left(\nabla h_{i j}\right)^{2}\right. \\
& \left.+\frac{32 f_{9}}{M^{2}} h_{i j}^{\prime} \nabla^{2} h_{i j}^{\prime}+\frac{32 f_{9}}{M^{2}}\left(\nabla^{2} h_{i j}\right)^{2}\right] .
\end{aligned}
$$




\section{A.8 Weyl tilde squared}

Let us consider the following parity breaking operator as given in [80]

$$
\begin{aligned}
S_{\tilde{W} W}^{(2)} & =\frac{M_{P l}^{2}}{2} \int \mathrm{d}^{4} x \frac{f_{10}}{M^{2}} \epsilon^{\mu \nu \rho \sigma} g^{\kappa \eta} g^{\lambda \zeta} W_{\mu \nu \eta \zeta}^{(1)} W_{\rho \sigma \kappa \lambda}^{(1)} \\
& =\frac{M_{P l}^{2}}{2} \int \mathrm{d}^{4} x \frac{f_{10}}{M^{2}} \epsilon^{i j k 0} a^{-4}\left[4 W_{l m i j}^{(1)} W_{l m k 0}^{(1)}-8 W_{l 0 i j}^{(1)} W_{l 0 k 0}^{(1)}\right] .
\end{aligned}
$$

The Weyl tensor components as given in [137], relations (A.15 - A.17), are

$$
\begin{gathered}
W_{l 0 k 0}^{(1)}=-\frac{a}{2}\left(a h_{l k}^{\prime}\right)^{\prime}, \\
W_{l 0 i j}^{(1)}=-a^{2} h_{l[i, j]}^{\prime}=-\frac{a^{2}}{2}\left(h_{l i ; j}^{\prime}-h_{l j ; i}^{\prime}\right), \\
W_{l m k 0}^{(1)}=-a^{2} h_{l[k, m]}^{\prime}=-\frac{a^{2}}{2}\left(h_{l k ; m}^{\prime}-h_{l m ; k}^{\prime}\right), \\
W_{l m i j}^{(1)}=a\left[-\delta_{i[l}\left(a h_{m] j}^{\prime}\right)^{\prime}+\delta_{j[l}\left(a h_{m] i}^{\prime}\right)^{\prime}\right] \\
=\frac{a}{2}\left[-\delta_{i l}\left(a h_{m j}^{\prime}\right)^{\prime}+\delta_{i m}\left(a h_{l j}^{\prime}\right)^{\prime}+\delta_{j l}\left(a h_{m i}^{\prime}\right)^{\prime}-\delta_{j m}\left(a h_{l i}^{\prime}\right)^{\prime}\right],
\end{gathered}
$$

where we relabelled and placed all the indices down so that they match the notation in [80]. Then, at second-order in metric perturbations, we find that (A.8.1) becomes

$$
\begin{aligned}
S_{W^{2}}^{(2)} & =\frac{M_{P l}^{2}}{2} \int \mathrm{d}^{4} x \frac{f_{10}}{M^{2}} \epsilon^{i j k 0}\left[4 a^{-1} h_{m j ; i}^{\prime}\left(a h_{m k}^{\prime}\right)^{\prime}+4 a^{-1} h_{l j ; i}^{\prime}\left(a h_{l k}^{\prime}\right)^{\prime}\right] \\
& =\frac{M_{P l}^{2}}{2} \int \mathrm{d}^{4} x \frac{f_{10}}{M^{2}} 8 \epsilon^{i j k 0} a^{-1} h_{l j ; i}^{\prime}\left(a h_{l k}^{\prime}\right)^{\prime} .
\end{aligned}
$$

Using the equation of motion for tensor fluctuations in conformal spacetime as defined in (A.7.4). Then we can approximate (A.8.6) as

$$
\begin{aligned}
S_{W^{2}}^{(2)} & =\frac{M_{P l}^{2}}{2} \int \mathrm{d}^{4} x \frac{f_{10}}{M^{2}} 8 \epsilon^{i j k 0} h_{l j ; i}^{\prime}\left(\mathcal{H} h_{l k}^{\prime}+h_{l k}^{\prime \prime}\right) \\
& =\frac{M_{P l}^{2}}{2} \int \mathrm{d}^{4} x \frac{f_{10}}{M^{2}} 4 \epsilon^{i j k 0} h_{l j, i}^{\prime}\left(h_{l k}^{\prime \prime}+\nabla^{2} h_{l k}\right) \\
& =\frac{M_{P l}^{2}}{2} \int \mathrm{d}^{4} x \frac{f_{10}}{M^{2}} 2 \epsilon^{i j k 0} \partial_{\eta}\left[\left(h_{l j ; i}^{\prime} h_{l k}^{\prime}\right)+\left(h_{l i} \nabla^{2} h_{l k ; j}\right)\right] .
\end{aligned}
$$


Integrating by parts once with respect to conformal time and then integrating by parts the second term with respect to the spatial index $r$, gives the expression for the parity breaking term in [110], relation (3)

$$
\begin{aligned}
S_{W^{2}}^{(2)} & =-\frac{M_{P l}^{2}}{2} \int \mathrm{d}^{4} x \frac{f_{10}^{\prime}}{M^{2}} 2 \epsilon^{i j k 0} f\left[h_{q j ; i}^{\prime} h_{q k}^{\prime}+h_{q i} \nabla^{2} h_{q k ; j}\right] \\
& =-\frac{M_{P l}^{2}}{2} \int \mathrm{d}^{4} x \frac{f_{10}^{\prime}}{M^{2}} 2 \epsilon^{i j k 0}\left[h_{q j ; i}^{\prime} h_{q k}^{\prime}-h_{q i ; r} h_{q k ; j r}\right] .
\end{aligned}
$$

Finally, taking a factor of $1 / 4$ out, this can be written as

$$
S_{W^{2}}^{(2)}=-\frac{M_{P l}^{2}}{8} \int \mathrm{d}^{4} x \frac{f_{10}^{\prime}}{M^{2}} 8 \epsilon^{i j k 0}\left[h_{q j ; i}^{\prime} h_{q k}^{\prime}-h_{q i ; r} h_{q k ; j r}\right]
$$

Another approximation to (A.8.6) is to choose to integrate the $h_{i j}^{\prime \prime}$ term by parts, instead of using the leading-order equations of motion. In that case one gets

$$
\begin{aligned}
S_{W^{2}}^{(2)} & =\frac{M_{P l}^{2}}{2} \int \mathrm{d}^{4} x \frac{f_{10}}{M^{2}} 8 \epsilon^{i j k 0} a^{-1} h_{l j ; i}^{\prime}\left(a h_{l k}^{\prime}\right)^{\prime} \\
& \frac{M_{P l}^{2}}{2} \int \mathrm{d}^{4} x \frac{f_{10}}{M^{2}} 8 \epsilon^{i j k 0} h_{l j ; i}^{\prime}\left(\mathcal{H} h_{l k}^{\prime}+h_{l k}^{\prime \prime}\right) \\
& =\frac{M_{P l}^{2}}{2} \int \mathrm{d}^{4} x \frac{1}{M^{2}}\left(8 f_{10} \mathcal{H} h_{l j ; i}^{\prime} h_{l k}^{\prime}-4 f_{10}^{\prime} h_{l j ; i}^{\prime} h_{l k}^{\prime}\right) \\
& =\frac{M_{P l}^{2}}{2} \int \mathrm{d}^{4} x \frac{1}{M^{2}} \epsilon^{i j k 0}\left(8 f_{10} \mathcal{H}-4 f_{10}^{\prime}\right) h_{l j ; i}^{\prime} h_{l k}^{\prime} .
\end{aligned}
$$

In this case there is only one contribution coming from the parity violating term.

\section{A.9 Disformal transformation of linearised (NLO) operators}

Our starting point is the, most familiar to us, extension to Einstein's gravity as discussed in [80]. At the background level the action is given by

$$
S=\frac{M_{P l}^{2}}{2} \int \mathrm{d}^{4} x \sqrt{-g}\left[R+\frac{f_{1}}{\Lambda^{2}} W^{\mu \nu \rho \sigma} W_{\mu \nu \rho \sigma}+\frac{f_{2}}{\Lambda^{2}} \epsilon^{\mu \nu \rho \sigma} g^{\kappa \beta} g^{\lambda \zeta} W_{\mu \nu \beta \zeta} W_{\rho \sigma \kappa \lambda}\right]
$$

This is expanded around an FRW background to second-order in perturbations of the metric (see Appendices (A.6) and (A.7), (A.8)). We choose to express the linearised action in term of barred parameters. The action reads 


$$
\begin{aligned}
S^{(2)} & =\int \mathrm{d}^{3} x \mathrm{~d} \bar{\eta}\left\{\frac{M_{P l}^{2}}{8} \bar{a}^{2}\left[\left(\partial_{\bar{\eta}} h_{i j}\right)^{2}-\left(\partial_{k} h_{i j}\right)^{2}\right]\right. \\
& +\frac{M_{P l}^{2}}{4 \Lambda^{2}} \bar{f}_{1}\left[\left(\partial_{\bar{\eta}}\left(\partial_{\bar{\eta}} h_{i j}\right)+\nabla^{2} h_{i j}\right)^{2}-4\left(\partial_{k} \partial_{\bar{\eta}} h_{i j}\right)^{2}\right] \\
& \left.-\frac{M_{P l}^{2}}{2 \Lambda^{2}} 8 \bar{f}_{2} \epsilon^{i j k}\left(\partial_{\bar{\eta}} \partial_{i} h_{l j}\right)\left[\overline{\mathcal{H}}\left(\partial_{\bar{\eta}} h_{l k}\right)+\partial_{\bar{\eta}}\left(\partial_{\bar{\eta}} h_{l k}\right)\right]\right\},
\end{aligned}
$$

where $\bar{f}_{1}$ and $\bar{f}_{2}$ are dimensionless functions of time. We introduce modifications to gravity by performing an inverse disformal transformation following the prescription in $[133,135,136]$.

When dealing with only (NLO) operators one can employ a suitable field redefinition to ensure second-order equations of motion. There are two ways to do this here. One is to first transform the action and then use the equations of motion of the leading order contributions to the quadratic action in the new frame to reduce the order of the time derivatives. Equivalently one can use the leading order equations of motion in the barred frame to reduce the order of the time derivatives and then transform the action to the new frame. The result will be the same. In this Appendix we choose the latter. Therefore, using the equations of motion for the quadratic action in the Einstein frame, namely

$$
\partial_{\bar{\eta}}\left(\partial_{\bar{\eta}} h_{i j}\right)+2 \overline{\mathcal{H}} \partial_{\bar{\eta}} h_{i j}-\nabla^{2} h_{i j}=0
$$

the action reduces to

$$
\begin{aligned}
S^{(2)} & =\int \mathrm{d}^{3} x \mathrm{~d} \bar{\eta}\left\{\frac{M_{P l}^{2}}{8} \bar{a}^{2}\left[\left(\partial_{\bar{\eta}} h_{i j}\right)^{2}-\left(\partial_{k} h_{i j}\right)^{2}\right]\right. \\
& +\frac{M_{P l}^{2}}{\Lambda^{2}}\left[\bar{f}_{1} \overline{\mathcal{H}}^{2}\left(\partial_{\bar{\eta}} h_{i j}\right)^{2}-\left[\left(\partial_{\bar{\eta}} \bar{f}_{1}\right) \overline{\mathcal{H}}+\bar{f}_{1}\left(\partial_{\bar{\eta}} \overline{\mathcal{H}}\right)\right]\left(\nabla h_{i j}\right)^{2}-\bar{f}_{1}\left(\partial_{\bar{\eta}} \nabla h_{i j}\right)^{2}+\bar{f}_{1}\left(\nabla^{2} h_{i j}\right)^{2}\right] \\
& \left.-\frac{M_{P l}^{2}}{\Lambda_{C S}}\left(\partial_{\bar{\eta}} \bar{f}_{2}\right) \epsilon^{i j k}\left[\left(\partial_{\bar{\eta}} h_{q i}\right)\left(\partial_{\bar{\eta}} \partial_{j} h_{k q}\right)+\left(\nabla^{2} h_{q i}\right) \partial_{j} h_{k q}\right]\right\} .
\end{aligned}
$$

We choose the following redefinition of the time-coordinate and the scale factor

$$
\mathrm{d} \bar{\eta}=c_{T} \mathrm{~d} \eta, \quad \bar{a}=c_{T}^{-\frac{1}{2}} \mathcal{F}^{\frac{1}{2}} a
$$

where $c_{T}=\mathcal{F} \mathcal{G}^{-1}$ and with the parameters $\mathcal{F}, \mathcal{G}$ being functions of time. We find the Hubble parameter transforms as

$$
\overline{\mathcal{H}}=c_{T}^{-1}\left(\mathcal{H}-\frac{1}{2} \frac{c_{T}^{\prime}}{c_{T}}+\frac{1}{2} \frac{\mathcal{F}^{\prime}}{\mathcal{F}}\right) .
$$


In the new frame the action becomes

$$
\begin{aligned}
S^{(2)} & =\frac{M_{P l}^{2}}{8} \int \mathrm{d}^{3} x \mathrm{~d} \eta a^{2}\left\{\left(\mathcal{G}+\frac{8 \bar{f}_{1} \mathcal{A}}{c_{T}^{3} a^{2} \Lambda^{2}}\right)\left(h_{i j}^{\prime}\right)^{2}-\left[\mathcal{F}+\frac{8\left(\bar{f}_{1}^{\prime} \mathcal{B}_{1}+\bar{f}_{1} \mathcal{B}_{2}\right)}{c_{T} a^{2} \Lambda^{2}}\right]\left(\nabla h_{i j}\right)^{2}\right. \\
& -\frac{8 \bar{f}_{1}}{c_{T} a^{2} \Lambda^{2}}\left(\nabla h_{i j}^{\prime}\right)^{2}+\frac{8 \bar{f}_{1} c_{T}}{a^{2} \Lambda^{2}}\left(\nabla^{2} h_{i j}\right)^{2} \\
& \left.-\frac{8 \bar{f}_{2}^{\prime}}{a^{2} \Lambda^{2}} \epsilon^{i j k}\left[\frac{1}{c_{T}^{2}} h_{q i}^{\prime} \partial_{j} h_{k q}^{\prime}-\left(\partial^{r} h^{q}{ }_{i}\right) \partial_{j} \partial_{r} h_{k q}\right]\right\}
\end{aligned}
$$

where we denote derivatives with respect to the conformal as ${ }^{\prime}=\partial_{\eta}$ and have defined the following parameters

$$
\begin{aligned}
& \mathcal{A}=\mathcal{H}^{2}+\mathcal{H}\left(\frac{\mathcal{F}^{\prime}}{\mathcal{F}}-\frac{c_{T}^{\prime}}{c_{T}}\right)+\left(\frac{1}{2} \frac{\mathcal{F}^{\prime}}{\mathcal{F}}-\frac{1}{2} \frac{c_{T}^{\prime}}{c_{T}}\right)^{2}, \\
& \mathcal{B}_{1}=\mathcal{H}+\frac{1}{2} \frac{\mathcal{F}^{\prime}}{\mathcal{F}}-\frac{1}{2} \frac{c_{T}^{\prime}}{c_{T}}, \quad \mathcal{B}_{2}=\mathcal{H}^{\prime}-\mathcal{H} \frac{c_{T}^{\prime}}{c_{T}}-\frac{1}{2} \frac{c_{T}^{\prime}}{c_{T}} \frac{\mathcal{F}^{\prime}}{\mathcal{F}}+\left(\frac{c_{T}^{\prime}}{c_{T}}\right)^{2}-\frac{1}{2}\left(\frac{\mathcal{F}^{\prime}}{\mathcal{F}}\right)^{2}+\frac{1}{2} \frac{\mathcal{F}^{\prime \prime}}{\mathcal{F}}-\frac{1}{2} \frac{c_{T}^{\prime \prime}}{c_{T}} .
\end{aligned}
$$

We do not know yet how the functions $\bar{f}_{1}$ and $\bar{f}_{2}$ transform. At the limit of constant parameter $c_{T}$ the parameters $\mathcal{A}, \mathcal{B}_{1}$ and $\mathcal{B}_{2}$ reduce to

$$
\mathcal{A}=\mathcal{H}^{2}+\mathcal{H} \frac{\mathcal{F}^{\prime}}{\mathcal{F}}+\frac{1}{4}\left(\frac{\mathcal{F}^{\prime}}{\mathcal{F}}\right)^{2}, \quad \mathcal{B}_{1}=\mathcal{H}+\frac{1}{2} \frac{\mathcal{F}^{\prime}}{\mathcal{F}}, \quad \mathcal{B}_{2}=\mathcal{H}^{\prime}-\frac{1}{2}\left(\frac{\mathcal{F}^{\prime}}{\mathcal{F}}\right)^{2}+\frac{1}{2} \frac{\mathcal{F}^{\prime \prime}}{\mathcal{F}}
$$

In the case where $\mathcal{F}, \mathcal{G}$ are constants we simply have

$$
\mathcal{A}=\mathcal{H}^{2}, \quad \mathcal{B}_{1}=\mathcal{H}, \quad \mathcal{B}_{2}=\mathcal{H}^{\prime}
$$

i.e. the Hubble parameter simply transforms as a derivative $\overline{\mathcal{H}}=c_{T}^{-1} \mathcal{H}$. We proceed to produce the equations of motion. The Fourier transform for tensor perturbations is given by

$$
h_{i j}(\mathbf{x}, \eta)=\frac{1}{(2 \pi)^{\frac{3}{2}}} \int \mathrm{d} \mathbf{k} \sum_{s=R, L} p_{i j}^{s}(\mathbf{k}) h^{s}(\mathbf{k}, \eta) e^{i \mathbf{k} \cdot \mathbf{x}}
$$

where the tensor polarizations are defined in a circular basis as

$$
p_{i j}^{R} \equiv \frac{1}{\sqrt{2}}\left(p_{i j}^{+}+i p_{i j}^{\times}\right), \quad \text { and } \quad p_{i j}^{L} \equiv \frac{1}{\sqrt{2}}\left(p_{i j}^{+}-i p_{i j}^{\times}\right)=\left(p_{i j}^{R}\right)^{*},
$$


where $p_{i j}^{+}, p_{i j}^{\times}$are the two linear polarization tensors and $p_{i j}^{R}, p_{i j}^{L}$ are polarizations that rotate in the right $(R)$ and left $(L)$ handed directions, respectively. These satisfy the following transversality and traceless conditions

$$
p_{i j}^{s} k_{j}=0, \quad \text { and } \quad\left(p_{i}^{i}\right)^{s}=0, \quad s=L, R,
$$

where $\mathbf{k}=k_{i}$ are momenta in the spatial directions. In momentum space the EulerLagrange equations read $^{1}$

$$
\begin{aligned}
& \left(h^{s}\right)^{\prime \prime}\left(1+\frac{8 \bar{f}_{1} \mathcal{A}}{c_{T}^{3} \mathcal{G} a^{2} \Lambda^{2}}-\frac{8 k^{2} \bar{f}_{1}}{c_{T} \mathcal{G} a^{2} \Lambda^{2}}-\frac{8 k \lambda^{s} \bar{f}_{2}^{\prime}}{c_{T}^{2} \mathcal{G} a^{2} \Lambda^{2}}\right) \\
+ & \left(h^{s}\right)^{\prime}\left(2 \mathcal{H}+\frac{8 \bar{f}_{1} \mathcal{A}^{\prime}}{c_{T}^{3} \mathcal{G} a^{2} \Lambda^{2}}-\frac{24 \bar{f}_{1} \mathcal{A} c_{T}^{\prime}}{c_{T}^{4} \mathcal{G} a^{2} \Lambda^{2}}+\frac{\mathcal{G}^{\prime}}{\mathcal{G}}+\frac{8 \bar{f}_{1}^{\prime} \mathcal{A}}{c_{T}^{3} \mathcal{G} a^{2} \Lambda^{2}}\right. \\
- & \left.\frac{8 k^{2} \bar{f}_{1}^{\prime}}{c_{T} \mathcal{G} a^{2} \Lambda^{2}}+\frac{8 k^{2} \bar{f}_{1} c_{T}^{\prime}}{c_{T}^{2} \mathcal{G} a^{2} \Lambda^{2}}-\frac{8 k \lambda^{s} \bar{f}_{2}^{\prime \prime}}{c_{T}^{2} \mathcal{G} a^{2} \Lambda^{2}}+\frac{16 k \lambda^{s} \bar{f}_{2}^{\prime} c_{T}^{\prime}}{c_{T}^{3} \mathcal{G} a^{2} \Lambda^{2}}\right) \\
+ & k^{2} h^{s}\left(c_{T}^{2}+\frac{8 \bar{f}_{1}^{\prime} \mathcal{B}_{1}}{c_{T} \mathcal{G} a^{2} \Lambda^{2}}+\frac{8 \bar{f}_{1} \mathcal{B}_{2}}{c_{T} \mathcal{G} a^{2} \Lambda^{2}}-\frac{8 k^{2} \bar{f}_{1} c_{T}}{\mathcal{G} a^{2} \Lambda^{2}}-\frac{8 k \lambda^{s} \bar{f}_{2}^{\prime}}{\mathcal{G} a^{2} \Lambda^{2}}\right)=0,
\end{aligned}
$$

where we have used the following identity to simplify the result

$$
i \frac{k_{p}}{k} \epsilon^{p j k} p_{i k}=-\lambda^{s}\left(p_{i}^{j}\right)^{s}, \quad \lambda^{s}= \pm 1,
$$

and have omitted summation over left and right modes. Next, for simplicity we consider the case where $c_{T}=$ const. and $\mathcal{F}, \mathcal{G}$ are functions of time as in (A.9.9). We can obtain the evolution equations for tensor fluctuations in momentum space in terms of the canonically normalized amplitudes

$$
\mu^{s}=\frac{M_{P l}}{2} h^{s} z^{s}
$$

We find

$$
\left(\mu^{s}\right)^{\prime \prime}+\left[k^{2} \frac{\left(c_{T}^{2}+\frac{8\left(\bar{f}_{1} \mathcal{B}_{2}+\bar{f}_{1}^{\prime} \mathcal{B}_{1}\right)}{c_{T} \mathcal{G} a^{2} \Lambda^{2}}-\frac{8 c_{T} k^{2} \bar{f}_{1}}{\mathcal{G} a^{2} \Lambda^{2}}-\frac{8 \lambda^{s} k \bar{f}_{2}^{\prime}}{\mathcal{G} a^{2} \Lambda^{2}}\right)}{\left(1+\frac{8 \bar{f}_{1} \mathcal{A}}{c_{T}^{3} \mathcal{G} a^{2} \Lambda^{2}}-\frac{8 k^{2} \bar{f}_{1}}{c_{T} \mathcal{G} a^{2} \Lambda^{2}}-\frac{8 \lambda^{s} k \bar{f}_{2}^{\prime}}{c_{T}^{2} \mathcal{G} a^{2} \Lambda^{2}}\right)}-\frac{\left(z^{s}\right)^{\prime \prime}}{z^{s}}\right] \mu^{s}=0,
$$

where the effective potential is defined in terms of

$$
z^{s}=z_{1}(\tau) \sqrt{1+\frac{8 \bar{f}_{1} \mathcal{A}}{c_{T}^{3} \mathcal{G} a^{2} \Lambda^{2}}-\frac{8 k^{2} \bar{f}_{1}}{c_{T} \mathcal{G} a^{2} \Lambda^{2}}-\frac{8 \lambda^{s} k \bar{f}_{2}^{\prime}}{c_{T}^{2} \mathcal{G} a^{2} \Lambda^{2}}}, \quad z_{1}(\tau)=z_{0} a \sqrt{2 \mathcal{G} c_{T}^{3}} \Lambda
$$

\footnotetext{
${ }^{1}$ We have dropped, for brevity, the $\mathbf{k}, \eta$ dependencies.
} 
and it is given by

$$
\begin{aligned}
\frac{\left(z^{s}\right)^{\prime \prime}}{z^{s}} & =\frac{z_{1}^{\prime \prime}}{z_{1}}+\frac{z_{1}^{\prime}}{z_{1}}\left[\frac{\left(\frac{8 \bar{f}_{1} \mathcal{A}}{c_{T}^{3} \mathcal{G} a^{2} \Lambda^{2}}\right)^{\prime}-k^{2}\left(\frac{8 \bar{f}_{1}}{c_{T} \mathcal{G} a^{2} \Lambda^{2}}\right)^{\prime}-k \lambda^{s}\left(\frac{8 \bar{f}_{2}^{\prime}}{c_{T}^{2} \mathcal{G} a^{2} \Lambda^{2}}\right)^{\prime}}{1+\frac{8 \bar{f}_{1} \mathcal{A}}{c_{T}^{3} \mathcal{G} a^{2} \Lambda^{2}}-\frac{8 k^{2} \bar{f}_{1}}{c_{T} \mathcal{G} a^{2} \Lambda^{2}}-\frac{8 \lambda^{s} k \bar{f}_{2}^{\prime}}{c_{T}^{2} \mathcal{G} a^{2} \Lambda^{2}}}\right] \\
& +\frac{1}{2}\left[\frac{\left(\frac{8 \bar{f}_{1} \mathcal{A}}{c_{T}^{3} \mathcal{G} a^{2} \Lambda^{2}}\right)^{\prime \prime}-k^{2}\left(\frac{8 \bar{f}_{1}}{c_{T} \mathcal{G} a^{2} \Lambda^{2}}\right)^{\prime \prime}-k \lambda^{s}\left(\frac{8 \bar{f}_{2}^{\prime}}{c_{T}^{2} \mathcal{G} a^{2} \Lambda^{2}}\right)^{\prime \prime}}{1+\frac{8 \bar{f}_{1} \mathcal{A}}{c_{T}^{3} \mathcal{G} a^{2} \Lambda^{2}}-\frac{8 k^{2} \bar{f}_{1}}{c_{T} \mathcal{G} a^{2} \Lambda^{2}}-\frac{8 \lambda^{s} k \bar{f}_{2}^{\prime}}{c_{T}^{2} \mathcal{G} a^{2} \Lambda^{2}}}\right]^{\prime} \\
& -\frac{1}{4}\left[\frac{\left(\frac{8 \bar{f}_{1} \mathcal{A}}{c_{T}^{3} \mathcal{G} a^{2} \Lambda^{2}}\right)^{\prime}-k^{2}\left(\frac{8 \bar{f}_{1}}{c_{T} \mathcal{G} a^{2} \Lambda^{2}}\right)^{\prime}-k \lambda^{s}\left(\frac{8 \bar{f}_{2}^{\prime}}{c_{T}^{2} \mathcal{G} a^{2} \Lambda^{2}}\right)^{\prime}}{1+\frac{8 \bar{f}_{1} \mathcal{A}}{c_{T}^{3} \mathcal{G} a^{2} \Lambda^{2}}-\frac{8 k^{2} \bar{f}_{1}}{c_{T} \mathcal{G} a^{2} \Lambda^{2}}-\frac{8 \lambda^{s} k \bar{f}_{2}^{\prime}}{c_{T}^{2} \mathcal{G} a^{2} \Lambda^{2}}},\right.
\end{aligned}
$$

with

$$
\frac{z_{1}^{\prime \prime}}{z_{1}}=\frac{a^{\prime \prime}}{a}+\frac{1}{2} \frac{\mathcal{G}^{\prime \prime}}{\mathcal{G}}+\frac{a^{\prime}}{a} \frac{\mathcal{G}^{\prime}}{\mathcal{G}}-\frac{1}{4}\left(\frac{\mathcal{G}^{\prime}}{\mathcal{G}}\right)^{2}, \quad \frac{z_{1}^{\prime}}{z_{1}}=\frac{a^{\prime}}{a}+\frac{1}{2} \frac{\mathcal{G}^{\prime}}{\mathcal{G}}
$$

To ensure correct signs for the kinetic term and a healthy speed for gravitons we define the following constraints

$$
\begin{gathered}
\left(z^{s}\right)^{2}>0 \Rightarrow 1+\frac{8 \bar{f}_{1} \mathcal{A}}{c_{T}^{3} \mathcal{G} a^{2} \Lambda^{2}}-\frac{8 k^{2} \bar{f}_{1}}{c_{T} \mathcal{G} a^{2} \Lambda^{2}}-\frac{8 \lambda^{s} k \bar{f}_{2}^{\prime}}{c_{T}^{2} \mathcal{G} a^{2} \Lambda^{2}}>0 \\
\text { and } \quad c_{T}^{2}+\frac{8\left(\bar{f}_{1} \mathcal{B}_{2}+\bar{f}_{1}^{\prime} \mathcal{B}_{1}\right)}{c_{T} \mathcal{G} a^{2} \Lambda^{2}}-\frac{8 c_{T} k^{2} \bar{f}_{1}}{\mathcal{G} a^{2} \Lambda^{2}}-\frac{8 \lambda^{s} k \bar{f}_{2}^{\prime}}{\mathcal{G} a^{2} \Lambda^{2}}>0
\end{gathered}
$$

\section{A.10 The equations of motion}

The equation to solve is

$$
\left(\mu^{s}\right)^{\prime \prime}+\left[-\frac{1}{4}+\frac{i g_{10} \lambda^{s}}{2 c_{T} \chi}+\frac{(-2)}{\chi^{2}}\right] \mu^{s}=0
$$

where

$$
\chi=2 i c_{T} k \eta,
$$

and $\mu=\mu(\mathbf{k}, \eta)$. Solutions are in terms of Whittaker functions. Here we keep the Whittaker $W$ function which has a growing solution for left modes and a negative decaying solution for right modes. The Whittaker $W$ solution is expressed as [270] (page 1024, relation $9.220,2$ ) 


$$
W_{\nu, \mu}(\chi)=\chi^{\mu+\frac{1}{2}} e^{-\frac{\chi}{2}} U\left(\mu-\nu+\frac{1}{2}, 2 \mu+1 ; \chi\right),
$$

where $U$ is the confluent hypergeometric function. In our case we have that

$$
\nu=\frac{i g_{10} \lambda^{s}}{2 c_{T}}
$$

and $\mu$ is found to be $\frac{1}{4}-\mu^{2}=-2 \Rightarrow \mu^{2}=\left(2+\frac{1}{4}\right)=\frac{9}{4} \Rightarrow \mu= \pm \frac{3}{2}$. Any root works, therefore, we choose

$$
\mu=\frac{3}{2}
$$

The full solution is given by

$$
\mu^{s}=B_{1}(k) W_{\nu, \mu}(\chi)=B_{1}(k) e^{-i c_{T} k \eta}\left(2 i c_{T} k \eta\right)^{2} U\left(2-\frac{i g_{10} \lambda^{s}}{2 c_{T}}, 4 ; 2 i c_{T} k \eta\right)
$$

which is normalized against the Bunch-Davies vacuum as follows. The asymptotic representation for the Whittaker function for large values of $|\chi|$ is given by (see [270] page 1026, relation 9.227 )

$$
\lim _{|\chi| \rightarrow \infty} W_{\nu, \mu}(\chi) \sim e^{-\frac{\chi}{2}} \chi^{\nu}
$$

Therefore, taking the small scale limit of the Whittaker function gives

$$
\begin{aligned}
\lim _{|k \eta| \rightarrow \infty} \mu^{s} & =B_{1}(k) \lim _{|k \eta| \rightarrow \infty} W_{\nu, \mu}(\chi)=B_{1}(k) e^{-i c_{T} k \eta}\left(i c_{T} k \eta\right) \frac{i g_{10} \lambda^{s}}{2 c_{T}} \\
& =B_{1}(k) e^{-i c_{T} k \eta_{e}\left[\frac{i g_{10} \lambda^{s}}{2 c_{T}} \ln \left(2 i c_{T} k \eta\right)\right]} \\
& =B_{1}(k) e^{-i c_{T} k \eta_{e}}\left\{\frac{i g_{10} \lambda^{s}}{2 c_{T}}\left[\ln \left(2 c_{T}|k \eta|\right)-i \frac{\pi}{2}\right]\right\} \\
& =B_{1}(k) e^{-i c_{T} k \eta_{e}\left(\frac{\pi g_{10} \lambda^{s}}{4 c_{T}}\right)}
\end{aligned}
$$

up to a phase. To correctly normalize the solutions we need to compare the above to the Bunch-Davies vacuum solution (4.5.12). We have 


$$
\lim _{|k \eta| \rightarrow \infty} \mu^{s}=-\frac{e^{-i c_{T} k \eta}}{\sqrt{2 c_{T} k}}=B_{1}(k) e^{-i c_{T} k \eta_{e}}\left(\frac{\pi g_{10} \lambda^{s}}{4 c_{T}}\right) .
$$

Finally, the normalization is

$$
B 1(k)=-\frac{1}{\sqrt{2 c_{T} k}} e^{\left(-\frac{\pi g_{10} \lambda^{s}}{4 c_{T}}\right)} .
$$

Substituting for $B_{1}(k)$ into (A.10.6) we find that the correctly normalized Whittaker solutions are given by

$$
\mu^{s}=\left(-2 c_{T} k \eta\right)^{\frac{3}{2}} \sqrt{-\eta} e^{-\frac{\pi \lambda^{s} g_{10}}{4 c_{T}}} U\left(2-\frac{i \lambda^{s} g_{10}}{2 c_{T}}, 4,2 i c_{T} k \eta\right) .
$$

To find the form of the solution at large scales we use (see [271], page 508, relation 13.5.6) $\lim _{z \rightarrow 0} U(a, b, z)=\frac{\Gamma(b-1)}{\Gamma(a)} z^{1-b}, \mathcal{R} b \geq 2, b \neq 2$, giving

$$
\lim _{|k \eta| \rightarrow 0} \mu^{s}=\sqrt{\frac{-\eta}{2\left(-c_{T} k \eta\right)^{3}}} \frac{1}{\Gamma\left(2-\frac{i g_{10} \lambda^{s}}{2 c_{T}}\right)} e^{-\frac{\pi \lambda^{s} g_{10}}{4 c_{T}}} .
$$

We need to find the power spectrum in terms of $h^{s}=2 \mu^{s}\left(z^{s} M_{P l}\right)^{-1}$. We first need to solve for

$$
\frac{\left(z^{s}\right)^{\prime \prime}}{z^{s}}=\frac{2}{\eta^{2}}+\frac{g_{10} k \lambda^{s}}{\eta}
$$

which has the general form (see [272], relation 2.8.24)

$$
\frac{W^{\prime \prime}}{W}=\frac{u^{2}}{4 \xi}+\frac{\left(\nu^{2}-1\right)}{4 \xi^{2}}+\frac{\psi(\xi)}{\xi},
$$

with $\nu= \pm 3, \psi(\xi)=0$. We pick the growing solution (for the left modes) to be

$$
z^{s}=2 z_{0} \sqrt{g_{10} \lambda^{s} k \eta} K_{3}\left(2 \sqrt{g_{10} \lambda^{s} k \eta}\right) .
$$

We use $\lim _{z \rightarrow 0} K_{n}(z)=\frac{1}{2} \Gamma(n)\left(\frac{1}{2} z\right)^{-n}$ to take the large scale limit giving

$$
\lim _{|k \eta| \rightarrow 0} z^{s}=z_{0} 2 \sqrt{g_{10} \lambda^{s} k \eta}\left(\sqrt{g_{10} \lambda^{s} k \eta}\right)^{-3}=-(H \eta)^{-1} .
$$

The tensor power spectrum, per polarization, is found to be

$$
P_{h}^{s}(k)=\frac{1}{\pi^{2}} \frac{H^{2}}{M_{P l}^{2} c_{T}^{3}}\left|\Gamma\left(2-\frac{i g_{10} \lambda^{s}}{2 c_{T}}\right)\right|^{-2} e^{-\frac{\pi \lambda^{s} g_{10}}{2 c_{T}}},
$$

where for $\lambda=-1$ the left modes are enhanced. 


\section{A.11 Disformal transformation of covariant (NLO) operators}

We consider a pure disformal transformation of the higher-curvature operators in [80]. Here we follow the procedure in the Appendix of [130]. Using the following field redefinition

$$
\pi=\int \mathrm{d} \phi \sqrt{B(\phi)}
$$

the barred metric can be expressed in terms of the following pure disformal relations

$$
\bar{g}_{\mu \nu}=g_{\mu \nu}+\pi_{, \mu} \pi_{, \nu}, \quad \bar{g}^{\mu \nu}=g^{\mu \nu}-\gamma_{0}^{2} \pi^{, \mu} \pi^{, \nu}
$$

with

$$
\gamma_{0}^{2}=\frac{1}{1-2 X_{\pi}}, \quad \nabla_{\mu} \gamma_{0}=-\gamma_{0}^{3} \pi^{, \alpha} \pi_{; \alpha \mu}, \quad X=-\frac{1}{2} \pi_{\mu} \pi^{, \mu}
$$

where we use the notation $\pi_{, \mu}=\partial_{\mu} \pi$ and $\pi_{\mu}^{; \mu}=\nabla_{\mu} \nabla^{\mu} \pi$. The action to transform was given in (4.3.1). The transformation of the Einstein-Hilbert term was already considered in [130]. Next we consider the transformation of the Weyl squared tensor which we express as

$$
\bar{W}_{\mu \nu \rho \sigma} \bar{W}^{\mu \nu \rho \sigma}=\bar{R}_{\mu \nu \rho \sigma} \bar{R}^{\mu \nu \rho \sigma}-2 \bar{R}_{\mu \nu} \bar{R}^{\mu \nu}+\frac{1}{3} \bar{R}^{2}
$$

We find the following contributions, where we denote the Weyl squared tensor as $W^{2}$ for short 


$$
\begin{aligned}
\bar{W}^{2} & =W_{\alpha \beta \delta \epsilon} W^{\alpha \beta \delta \epsilon} \\
& +4 \gamma_{0}^{2}\left\{R_{\alpha \delta \beta \epsilon} \Pi^{\beta \alpha} \Pi^{\epsilon \delta}-\left\langle R_{\alpha}^{\delta \epsilon \zeta} R_{\beta \delta \epsilon \zeta}\right\rangle+\left\langle R^{\delta \epsilon} R_{\alpha \delta \beta \epsilon}\right\rangle+R^{\alpha \beta} \Pi_{\delta \beta} \Pi_{\alpha}^{\delta}\right. \\
& \left.+\left\langle R_{\alpha}^{\delta} R_{\beta \delta}\right\rangle-\frac{1}{3} R\left\langle R_{\alpha \beta}\right\rangle-[\Pi] R^{\alpha \beta} \Pi+\frac{1}{6} R\left([\Pi]^{2}-\left[\Pi^{2}\right]\right)\right\} \\
& +4 \gamma_{0}^{4}\left\{\frac{1}{2}\left\langle\left\langle R_{\alpha}^{\zeta}{ }_{\beta}^{\eta} R_{\delta \zeta \epsilon \eta}\right\rangle\right\rangle-\left\langle R_{\alpha \epsilon \beta \zeta} \Pi^{\epsilon \delta} \Pi_{\delta}^{\zeta}\right\rangle+\left\langle R_{\alpha \epsilon \beta \zeta} \Pi^{\zeta \epsilon}\right\rangle\right. \\
& -4\left\langle R_{\beta \epsilon \delta \zeta} \Pi^{\delta}{ }_{\alpha} \Pi^{\zeta \epsilon}\right\rangle-2\left\langle R_{\alpha}^{\delta} \Pi_{\epsilon \delta} \Pi^{\epsilon \beta}\right\rangle+\frac{1}{3}\left\langle R_{\alpha \beta}\right\rangle\left(\left[\Pi^{2}\right]-[\Pi]^{2}\right) \\
& +2\left\langle R_{\alpha}^{\delta} \Pi_{\delta \beta}\right\rangle[\Pi]-\left\langle R^{\delta \epsilon} \Pi_{\delta \alpha} \Pi_{\epsilon \beta}\right\rangle+\langle\Pi\rangle R^{\epsilon \delta} \Pi-\frac{1}{6}\left\langle\left\langle R_{\alpha \beta} R_{\delta \epsilon}\right\rangle\right\rangle \\
& \left.+\frac{1}{3} R\left(\left\langle\Pi^{2}\right\rangle-\langle\Pi\rangle[\Pi]\right)+\frac{1}{12}[\Pi]^{4}-\frac{2}{3}[\Pi]^{2}\left[\Pi^{2}\right]+\frac{7}{12}\left[\Pi^{2}\right]^{2}+[\Pi]\left[\Pi^{3}\right]-\left[\Pi^{4}\right]\right\} \\
& +4 \gamma_{0}^{6}\left\{\langle\Pi\rangle\left\langle R_{\delta \zeta \epsilon \eta} \Pi^{\eta \zeta}\right\rangle-\left\langle\left\langle R_{\delta \zeta \epsilon \eta} \Pi_{\alpha}^{\zeta} \Pi^{\eta}{ }_{\beta}\right\rangle\right\rangle+\frac{1}{3}\langle R \alpha \beta\rangle\left(\langle\Pi\rangle^{2}-\langle\Pi\rangle[\Pi]\right)\right. \\
& +4\left\langle\Pi^{4}\right\rangle-3\left\langle\Pi^{3}\right\rangle[\Pi]+\frac{4}{3}\left\langle\Pi^{2}\right\rangle[\Pi]^{2}-\frac{1}{3}\langle\Pi\rangle[\Pi]^{3}-\frac{7}{3}\left\langle\Pi^{2}\right\rangle\left[\Pi^{2}\right] \\
& \left.+\frac{4}{3}\langle\Pi\rangle[\Pi]\left[\Pi^{2}\right]-\langle\Pi\rangle\left[\Pi^{3}\right]\right\} \\
& +\gamma_{0}^{8}\left\{\frac{4}{3}\left(\left\langle\Pi^{2}\right\rangle^{2}+\langle\Pi\rangle\left\langle\Pi^{2}\right\rangle[\Pi]\right)-\frac{2}{3}\langle\Pi\rangle^{2}[\Pi]^{2}-4\langle\Pi\rangle\left\langle\Pi^{3}\right\rangle+2\langle\Pi\rangle^{2}\left[\Pi^{2}\right]\right\} .
\end{aligned}
$$

Here we have condensed our expressions by using the Galileon notation in [130]. The Chern Simons gravitational term $W \tilde{W}$ can be expressed as

$$
\bar{W} \tilde{W}=-\bar{\varepsilon}^{\mu \nu \rho \sigma} \bar{R}_{\kappa \rho \sigma}^{\lambda} \bar{R}_{\lambda \mu \nu}^{\kappa}-4 \bar{\varepsilon}^{\lambda \mu \nu \rho} \bar{R}_{\kappa \lambda} \bar{R}_{\mu \nu \rho}^{\kappa}+\frac{2}{3} \bar{\varepsilon}^{\kappa \lambda \mu \nu} \bar{R} \bar{R}_{\kappa \lambda \mu \nu}
$$

where $\bar{\varepsilon}^{\mu \nu \rho \sigma}=\epsilon^{\mu \nu \rho \sigma} / \sqrt{|\bar{g}|}$ is the Levi-Civita tensor density. The above transforms as 


$$
\begin{aligned}
\bar{W} \tilde{W} & =\bar{\varepsilon}^{\mu \nu \rho \sigma} W_{\mu \nu \kappa \lambda} W_{\rho \sigma}^{\kappa \lambda} \\
& +4 \gamma_{0}^{2}\left\{\frac{1}{6} \bar{\varepsilon}^{\mu \nu \rho \sigma} R_{\mu \nu \rho \sigma}\left([\Pi]^{2}-\left[\Pi^{2}\right]\right)-\frac{1}{2}\left\langle\bar{\varepsilon}^{\nu \rho \sigma \alpha} R_{\kappa \mu \nu \rho} R_{\sigma \alpha}^{\lambda \mu}\right\rangle\right. \\
& +\left\langle\bar{\varepsilon}^{\mu \nu \rho \sigma} R_{\kappa \mu} R_{\lambda \nu \rho \sigma}\right\rangle+\left\langle\bar{\varepsilon}^{\nu \rho \sigma \alpha} R_{\kappa \mu \lambda \nu} R_{\rho \sigma \alpha}^{\mu}\right\rangle-\frac{1}{3}\left\langle\bar{\varepsilon}^{\mu \nu \rho \sigma} R_{\kappa \lambda} R_{\mu \nu \rho \sigma}\right\rangle \\
& \left.+\bar{\varepsilon}^{\mu \nu \rho \sigma} R_{\lambda \nu \rho \sigma} \Pi^{\lambda \kappa} \Pi_{\mu \kappa}-[\Pi] \bar{\varepsilon}^{\mu \nu \rho \sigma} R_{\lambda \nu \rho \sigma} \Pi_{\mu}{ }^{\lambda}+\bar{\varepsilon}^{\lambda \nu \rho \sigma} R_{\kappa \mu \rho \sigma} \Pi_{\lambda}{ }^{\kappa} \Pi_{\nu}{ }^{\mu}\right\} \\
& +4 \gamma_{0}^{4}\left\{\frac{1}{3} \bar{\varepsilon}^{\nu \rho \sigma \alpha} R_{\nu \rho \sigma \alpha}\left(\left\langle\Pi^{2}\right\rangle-\langle\Pi\rangle[\Pi]\right)+[\Pi]\left\langle\bar{\varepsilon}^{\mu \rho \sigma \alpha} R_{\lambda \rho \sigma \alpha} \Pi_{\mu \kappa}\right\rangle\right. \\
& -\left\langle\bar{\varepsilon}^{\nu \rho \sigma \alpha} R_{\mu \rho \sigma \alpha} \Pi^{\mu}{ }_{\kappa} \Pi_{\nu \lambda}\right\rangle-\left\langle\bar{\varepsilon}^{\nu \rho \sigma \alpha} R_{\lambda \rho \sigma \alpha} \Pi^{\mu}{ }_{\kappa} \Pi_{\nu \mu}\right\rangle \\
& \left.+\langle\Pi\rangle \bar{\varepsilon}^{\nu \rho \sigma \alpha} R_{\mu \rho \sigma \alpha} \Pi_{\nu}{ }^{\mu}-2\left\langle\bar{\varepsilon}^{\mu \rho \sigma \alpha} R_{\lambda \nu \sigma \alpha} \Pi_{\mu \kappa} \Pi^{\nu}{ }_{\rho}\right\rangle\right\} .
\end{aligned}
$$

From the above it is evident that the disformal transformation contributes extra pieces to the higher-curvature terms which results in altering the effective description. In the case of a general disformal transformation we would have to include several extra contributions. It is evident that these expressions can quickly grow to be very large. Here we focus on the transformation of linearised operators, which are simpler, and leave such considerations for future work.

\section{A.12 Next-to-next-to leading order operators}

Six-derivative parity preserving operators to scalar-tensor gravity were discussed in [126] while six-derivative parity-violating operators for scalar-tensor chiral theories were discussed in [128], where alongside the gravitational Chern Simons term they included first- and second-derivatives of the scalar field.

The usual prescription when working with EFTs is to write down the most general set of operators consistent with the symmetries of the full theory and use the background equations of motion to eliminate redundant ones as prescribed in [80]. At (NNLO) one may also have to use the first Bianchi identity to relate terms. So far, to our knowledge no one has produced the full set of non-redundant sixderivative corrections to gravity, in the context of single field inflation. Here we only tentatively look at some (NNLO) operators. As an example, let us briefly consider the following six-derivative operators

$$
\begin{aligned}
S_{N N L O}^{(0)} & =\frac{M_{P l}^{2}}{2} \int \mathrm{d}^{4} x \sqrt{-g}\left\{\frac{b_{1}}{\Lambda^{4}} W_{\mu \nu \rho \sigma} R^{\nu \sigma} \phi^{, \mu} \phi^{, \rho}+\frac{b_{2}}{\Lambda^{4}} W_{\mu \nu \rho \sigma} \phi^{; \mu \rho} \phi^{; \nu \sigma}\right. \\
& \left.+\epsilon^{\mu \nu \rho \sigma} \frac{d_{1}}{\Lambda^{4}} W_{\rho \sigma \kappa \lambda} R_{\nu}^{\lambda} \phi^{, \kappa} \phi^{, \mu}\right\} .
\end{aligned}
$$


Following the prescription in Section (4.3.1) and Appendix A.9 we disformally transform these operators, after we have expanded them to second-order in perturbations of the metric, to find the following contributions

$$
\begin{aligned}
S_{N N L O}^{(2)} & =\frac{M_{P l}^{2}}{2} \int \mathrm{d}^{4} x\left\{\frac { b _ { 1 } } { a ^ { 2 } \Lambda ^ { 4 } } \left[-\frac{\left(\phi^{\prime}\right)^{2}}{8 c_{T}^{6}}\left(h_{i j}^{\prime \prime}\right)^{2}-\frac{\mathcal{H}\left(\phi^{\prime}\right)^{2}}{4 c_{T}^{6}} h_{i j}^{\prime} h_{i j}^{\prime \prime}-\frac{\mathcal{H}\left(\phi^{\prime}\right)^{2}}{4 c_{T}^{5}} h_{i j}^{\prime} \nabla^{2} h_{i j}\right.\right. \\
& \left.+\frac{\left(\phi^{\prime}\right)^{2}}{8 c_{T}^{2}}\left(\nabla^{2} h_{i j}\right)^{2}\right]+\frac{b_{2}}{a^{2} \Lambda^{4}}\left[\left(\frac{\phi^{\prime} \phi^{\prime \prime}}{4 c_{T}^{6}}-\frac{\mathcal{H}\left(\phi^{\prime}\right)^{2}}{2 c_{T}^{6}}\right) h_{i j}^{\prime} h_{i j}^{\prime \prime}+\left(\frac{\phi^{\prime} \phi^{\prime \prime}}{4 c_{T}^{5}}-\frac{\mathcal{H}\left(\phi^{\prime}\right)^{2}}{2 c_{T}^{5}}\right) h_{i j}^{\prime} \nabla^{2} h_{i j}\right] \\
& \left.+\frac{2 d_{1}}{a^{2} \Lambda^{4}}\left[\frac{\left(\phi^{\prime}\right)^{2} \partial_{i} h_{l j}^{\prime} h_{l k}^{\prime \prime}}{c_{T}^{5}}+\frac{2 \mathcal{H}\left(\phi^{\prime}\right)^{2} \partial_{i} h_{l j}^{\prime} h_{l k}^{\prime}}{c_{T}^{5}}-\frac{\left(\phi^{\prime}\right)^{2} \partial_{i} h_{l j}^{\prime} \nabla^{2} h_{l k}}{c_{T}^{3}}\right]\right\} .
\end{aligned}
$$

The order of the energy expansion is $E^{4} / \Lambda_{*}^{4}$ where, using the arguments in Section (4.3.2), we can deduce that the effective mass scale is given by $\Lambda_{*}^{4}=c_{T}^{6} \Lambda^{4}$. It is easy to see that at the non-relativistic limit $c_{T} \ll 1$ these operators can pick up enhancements that could stand them relevant to the calculation. Therefore, it may become necessary to consider (NLO) and (NNLO) corrections to gravity. Suppose, we extend the action in (4.3.6) by including the contributions in (A.12.2). As we've already discussed in Section (4.3.3), when working with a combinations of (NLO) and (NNLO) terms, it is not entirely straightforward to find a field redefinition that can ensure second-order equations of motion.

Here we take a much more modest approach. At second-order in perturbations of the metric we expect the disformally transformed action to contain, schematically, the contributions shown in (4.3.17). We recall that this form of the action guarantees second-order equations of motion.

In the absence of a suitable field redefinition one may produce a theory that leads to at most second-order equations of motion by suitably choosing the free functions characterizing the higher derivative contributions. Of course, as these operators are motivated by a quantum mechanical description of gravity one would expect that such tuning may seem unnatural except if it is protected by some underlying symmetry. While this is true, we believe that there is no great loss of generality in doing things in the way indicated here as long as we maintain the form of the action shown in (4.3.17). Therefore, what follows should be understood as a naive approximation to a much more difficult problem.

The (NNLO) contributions have been chosen in such way so that the operator coupled to $b_{1}$ can be used to cancel the contribution $\left(h_{i j}^{\prime \prime}\right)^{2}$ in the Weyl squared tensor while the operators coupled to $b_{2}$ can be used to cancel the contribution $h_{i j}^{\prime} h_{i j}^{\prime \prime}$ in the operators coupled to $b_{1}$. Similarly, the operators coupled to $d_{1}$ can be used to cancel the contribution proportional to $\mathcal{H} h_{i j}^{\prime}$ in the gravitational Chern Simons term and introduce to the action a contribution proportional to $\nabla^{2} h_{l k}$. Therefore, with the following definitions 
$b_{1}=\frac{4 f_{1} \Lambda^{2} a^{2} c_{T}^{3}}{\left(\phi^{\prime}\right)^{2}}, \quad b_{2}=-\frac{b_{1} \mathcal{H} \phi^{\prime}}{2 \mathcal{H} \phi^{\prime}-\phi^{\prime \prime}}=-\frac{4 f_{1} \Lambda^{2} \mathcal{H} a^{2} c_{T}^{3}}{\phi^{\prime}\left(2 \mathcal{H} \phi^{\prime}-\phi^{\prime \prime}\right)}, \quad$ and $\quad d_{1}=-\frac{2 f_{2} \Lambda^{2} a^{2} c_{T}^{3}}{\left(\phi^{\prime}\right)^{2}}$,

the extended action reduces to

$$
\begin{aligned}
S^{(2)} & =\frac{M_{P l}^{2}}{8} \int \mathrm{d}^{3} x \mathrm{~d} \eta a^{2}\left\{\left(h_{i j}^{\prime}\right)^{2}-c_{T}^{2}\left(\nabla h_{i j}\right)^{2}-\frac{\omega_{1}}{a^{2} \Lambda^{2}}\left(\nabla h_{i j}^{\prime}\right)^{2}+\frac{\omega_{2}}{a^{2} \Lambda^{2}}\left(\nabla^{2} h_{i j}\right)^{2}\right. \\
& -\epsilon^{i j k}\left[\frac{g_{1}^{\prime}}{a^{2} \Lambda^{2}}\left(h^{q}{ }_{i}^{\prime}\left(\partial_{j} h_{k q}\right)^{\prime}-\frac{g_{2}^{\prime}}{a^{2} \Lambda^{2}}\left(\partial^{r} h^{q}{ }_{i}\right) \partial_{j} \partial_{r} h_{k q}\right]\right\} .
\end{aligned}
$$

In terms of (4.3.17) we can identify $\alpha=\beta=0, \gamma=\omega_{1}(a \Lambda)^{-2}, \delta=\omega_{2}(a \Lambda)^{-2}, \epsilon=$ $g_{1}^{\prime}(a \Lambda)^{-2}, \zeta=g_{2}^{\prime}(a \Lambda)^{-2}$, ensuring second-order equations of motion.

Note, here we do not consider operators of the form $\nabla_{\alpha} W_{\mu \nu \rho \sigma} \nabla^{\alpha} W^{\mu \nu \rho \sigma}$ which can have up to three derivatives acting on the metric. Such operators carry contributions to the equations motion with very high momenta which, for the sake of simplicity, we choose to ignore these terms in this work and only partially extend our low-energy action to the higher-derivative regime.

\section{A.13 Disformal transformation of the tensor ac- tion}

In [135] it was shown that a combination of conformal and disformal transformations allows one to recast the quadratic tensorial action into a form identical to the action of tensor modes in standard slow-roll inflation. See also [133] for an analysis of the consequences of disformal transformation for cosmological fluctuations. We discuss the implications of such transformations for our set-up.

The action for generalized G-inflation [36], $M_{P l}=1$, can be written as

$$
S=\frac{1}{8} \int \mathrm{d}^{3} x \mathrm{~d} t a^{3} \frac{\mathcal{F}_{T}}{c_{T}^{2}}\left[\left(\partial_{t} h_{i j}\right)^{2}-\frac{c_{T}^{2}}{a^{2}}\left(\nabla h_{i j}\right)^{2}\right]
$$

with $c_{T}^{2}=\frac{\mathcal{F}_{T}}{\mathcal{G}_{T}}$. Here we consider a disformal transformation of the form

$$
g_{\mu \nu} \rightarrow g_{\mu \nu}+\left(1-c_{T}^{2}\right) n_{\mu} n_{\nu}
$$

In [133] it was shown that a cosmological disformal transformation is equivalent to a rescaling of the time-coordinate. Under the disformal transformation (A.13.2), the FRW line-element becomes 


$$
\mathrm{d} \hat{s}^{2}=-c_{T}^{2} \mathrm{~d} t^{2}+a^{2} \mathrm{~d} x^{2}
$$

We see that the spatial part of the metric is left unaffected therefore, we can recover the form of the FRW line-element by simply transforming the time coordinate by $\mathrm{d} t=c_{T}^{-1} \mathrm{~d} \hat{t}$. This way the action becomes

$$
S=\frac{1}{8} \int \mathrm{d}^{3} x \mathrm{~d} \hat{t} a^{3} \frac{\mathcal{F}_{T}}{c_{T}}\left[\left(\partial_{t} h_{i j}\right)^{2}-\frac{1}{a^{2}}\left(\nabla h_{i j}\right)^{2}\right] .
$$

A conformal transformation of the form

$$
g_{\mu \nu} \rightarrow c_{T}^{-1} \mathcal{F}_{T} g_{\mu \nu}
$$

can bring the action into the standard Einstein-Hilbert form. It is convenient here to follow the prescription given in [135] and redefine the time-coordinate and the scale factor. Under the disformal and conformal transformations (A.13.2), (A.13.5) the FRW line-element reads $\mathrm{d} \hat{s}^{2}=c_{T}^{-1} \mathcal{F}_{T}\left(-c_{T}^{2} \mathrm{~d} t^{2}+a^{2} \mathrm{~d} x^{2}\right)$. Therefore, as before, we can recover the form of the FRW line-element, by defining the following

$$
\mathrm{d} t=c_{T}^{-\frac{1}{2}} \mathcal{F}_{\mathcal{T}}^{-\frac{1}{2}} \mathrm{~d} \hat{t}, \quad a=c_{T}^{\frac{1}{2}} \mathcal{F}_{\mathcal{T}}^{-\frac{1}{2}} \hat{a}
$$

With these redefinitions, the tensor perturbations are invariant under this transformation. The action now reads

$$
S=\frac{1}{8} \int \mathrm{d}^{3} x \mathrm{~d} \hat{t} \hat{a}^{3}\left[\left(\partial_{\hat{t}} h_{i j}\right)^{2}-\left(\nabla h_{i j}\right)^{2}\right]
$$

The Hubble parameter transforms as

$$
\hat{H}=\frac{1}{\hat{a}} \frac{\mathrm{d} \hat{a}}{\mathrm{~d} \hat{t}}=c_{T}^{-\frac{1}{2}} \mathcal{F}_{\mathcal{T}}{ }^{-\frac{1}{2}}\left[H+\frac{1}{2}\left(\frac{\dot{\mathcal{F}}_{\mathcal{T}}}{\mathcal{F}_{\mathcal{T}}}-\frac{\dot{c}_{T}}{c_{T}}\right)\right],
$$

for time-dependent parameters $\mathcal{F}_{\mathcal{T}}, \mathcal{G}_{\mathcal{T}}$. Here we use a dot to denote derivative with respect to the coordinate time $t$. From this we see that at leading order in the slow-roll parameters the Hubble parameter transforms as $\hat{H} \sim c_{T}^{-\frac{1}{2}} \mathcal{F}_{T}^{-\frac{1}{2}} H$.

We then use relations (5.5.23) and (5.5.24) to evaluate the right hand side of (A.13.8). When focussing on a slow-roll regime, we find, as expected, that the new Hubble parameter $\hat{H}$ is proportional to the original one. But when evaluated in the non-attractor phase, using the fact that $\dot{\phi}=\phi_{0} / a^{3}$ for a constant $\phi_{0}$, we find the following expression for the Hubble parameter in the Einstein frame 


$$
\hat{H}=-\frac{2 \sqrt{g_{t 2}} H a^{3}}{3 f_{t 2}\left|\phi_{0}\right|}=-\sqrt{\frac{4 H \phi_{0} g_{t 2}^{\frac{5}{4}}}{f_{t 2}^{\frac{3}{4}}}} \hat{a}^{-\frac{3}{2}}
$$

where in the second line we used the second relation in (A.13.6) between the scale factors in the two frames. Hence, in the Einstein frame where the tensor fluctuations are controlled by the action in (A.13.7), the background geometry in the non-attractor regime is described in terms of a dust dominated contracting Universe.

This implies that, within the Einstein frame description developed in this Appendix, the Universe undergoes a short phase of contraction - lasting a few e-folds during which the amplitude of tensor fluctuations can grow. This perspective offers another point of view for the results in the main text, within a frame where the action for quadratic tensor fluctuations is standard. It would be interesting to embed our scenario in a set-up with smooth transition between expanding and contracting phases, and study in detail the matching and stability issues for fluctuations. Possible instabilities in the bouncing transition phase can be tamed in sufficiently rich scalar-tensor systems related to the set-up we use in this work. A detailed analysis of this subject is beyond the scope of this article and we leave it for future investigations.

\section{A.14 The "GR" contribution}

Below we show the computation of the "GR" contribution. Here we evaluate the various parameters that appear throughout our arguments, explicitly. We can express the solution in (6.3.5) as

$$
h(\mathbf{k}, y)=\frac{e^{-i k y}\left(1-\frac{i}{k y}\right) y_{0}^{2}}{\sqrt{2 k} z_{0} y^{2}}
$$

where we express (5.6.3), in terms of the parameter $y$, as

$$
\tilde{z}_{T}=z_{0}\left(\frac{y}{y_{0}}\right)^{2}
$$

with

$$
z_{0}=-\frac{H_{0} \alpha_{1}}{6\left(-\rho_{1}+\delta_{1}+\beta_{1}+\sigma_{1}\right)}\left(g_{t} f_{t}\right)^{\frac{1}{4}} \quad \text { and } \quad y_{0}=-\frac{c_{T}}{H_{0}} .
$$

The leading order contribution at large scales is

$$
\lim _{y \rightarrow 0} h(\mathbf{k}, y)=-\frac{i y_{0}^{2}}{\sqrt{2} k^{\frac{3}{2}} z_{0} y^{3}} .
$$


We find that, to leading order, the power spectrum for each polarization is given by

$$
P_{h}=\frac{y_{0}^{4}}{2 k^{3} z_{0}^{2} y^{6}}
$$

Alternatively, this can be expressed directly in terms of (6.3.7), as

$$
P_{h}=\frac{2}{\pi^{2}} \frac{H^{2}}{\mathcal{F}_{T} c_{T}}=\frac{18}{\pi^{2}} \frac{\left(-\rho_{1}+\delta_{1}+\beta_{1}+\sigma_{1}\right)^{2}}{\alpha_{1}^{2} H_{0}^{6}} \frac{c_{T}^{5}}{f_{t}} y^{-6} .
$$

It is best to express these results in terms of the conformal time $\tau=y c_{T}^{-1}$ so that we can, later on, easily compare results with those in the Einstein frame. The solution in (A.14.1) becomes

$$
h(\mathbf{k}, \tau)=\frac{e^{-i k c_{T} \tau}\left(1-\frac{i}{k c_{T} \tau}\right) y_{0}^{2}}{\sqrt{2 k} z_{0} c_{T}^{2} \tau^{2}}=e^{-i c_{T} k \tau}\left(i-c_{T} k \tau\right) \frac{y_{0}^{2}}{\sqrt{2} k^{\frac{3}{2}}\left|z_{0}\right| c_{T}^{3}} \tau^{-3},
$$

and its conjugate

$$
h_{s}^{*}(\mathbf{k}, \tau)=-e^{i c_{T} k \tau}\left(i+c_{T} k \tau\right) \frac{y_{0}^{2}}{\sqrt{2} k^{\frac{3}{2}}\left|z_{0}\right| c_{T}^{3}} \tau^{-3} .
$$

It is useful to also include the time-derivative of the conjugate which is

$$
\partial_{\tau} h_{s}^{*}(k, \tau)=e^{i c_{T} k \tau} \frac{y_{0}^{2}}{\sqrt{2} k^{\frac{3}{2}}\left|z_{0}\right| c_{T}^{3}}\left(3 i+3 c_{T} k \tau-i c_{T}^{2} k^{2} \tau^{2}\right) \tau^{-4},
$$

where we have used that $z_{0}<0 \Rightarrow z_{0}=-\left|z_{0}\right|$. Then at large scales we have

$$
\lim _{\tau \rightarrow 0} h(\mathbf{k}, \tau)=\frac{i y_{0}^{2}}{\sqrt{2} k^{\frac{3}{2}}\left|z_{0}\right| c_{T}^{3} \tau^{3}}
$$

where

$$
\frac{y_{0}^{2}}{\left|z_{0}\right| c_{T}^{3}}=\left(\frac{g_{t}}{f_{t}^{3}}\right)^{\frac{1}{4}} \frac{6}{A_{1} H_{0}^{3}} .
$$

The power spectrum for each polarization, in conformal time, is evaluated to be

$$
P_{h}=\left(\frac{g_{t}}{f_{t}^{3}}\right)^{\frac{1}{2}} \frac{18}{A_{1}^{2} k^{3} H_{0}^{6} \tau^{6}}
$$


where $A_{1}=\frac{\alpha_{1}}{\left(-\rho_{1}+\delta_{1}+\beta_{1}+\sigma_{1}\right)}$.

Next, we calculate the three-point function for the GR term. Relation (2.5.6) becomes

$$
\begin{aligned}
\left\langle h^{3}\left(\tau_{e}\right)\right\rangle & =2 \operatorname{Re}\left\{-i h_{t m}\left(\mathbf{k}_{1}, \tau_{e}\right) h_{n r}\left(\mathbf{k}_{2}, \tau_{e}\right) h_{p v}\left(\mathbf{k}_{3}, \tau_{e}\right)\right. \\
& \times \int_{-\infty(1-i \epsilon)}^{\tau_{e}} \mathrm{~d} \tau \int \mathrm{d}^{3} x\left(-\frac{1}{4} a^{2} \mathcal{F}_{T}\right)\left[h^{j l}(\mathbf{x}, \tau) h^{i k}(\mathbf{x}, \tau)\right. \\
& \left.\left.-\frac{1}{2} h^{k l}(\mathbf{x}, \tau) h^{i j}(\mathbf{x}, \tau)\right] \partial_{k} \partial_{l} h_{i j}(\mathbf{x}, \tau)\right\}
\end{aligned}
$$

where the initial time $\tau_{0}$ is taken to be some early time in the far past when perturbations are still within the horizon $t_{0} \rightarrow-\infty$. The upper limit $\tau_{e}=\tau_{\text {end }}$ denotes the end of the second part of the evolution (in the usual case of slow-roll inflation, it would denote the end of inflation and therefore $\tau_{e} \rightarrow 0$ ).

Next, the tensor fluctuations $h_{i j}(\mathbf{x}, \tau)$ are decomposed into positive and negative frequency parts using (2.3.5), while the tensor fluctuations $h_{i j}\left(\mathbf{k}, \tau_{e}\right)$ are decomposed using (2.3.10). The modes inside the integral are contracted with the modes outside the integral. Only those that contain an even number of positive and negative modes survive. All other contractions (those that contain odd number of positive and negative modes) are vacuum expectation values and vanish (once we normal order them and apply (2.3.9)). We find the following contractions contribute to the first integral

$$
\begin{aligned}
& {\left[h_{t m}^{+}\left(\mathbf{k}_{1}, \tau_{e}\right), h_{j l}^{-}\left(-\mathbf{q}_{1}, \tau\right) e^{i \mathbf{q}_{1} \cdot \mathbf{x}}\right], \quad\left[h_{n r}^{+}\left(\mathbf{k}_{2}, \tau_{e}\right), h_{i k}^{-}\left(-\mathbf{q}_{2}, \tau\right) e^{i \mathbf{q}_{2} \cdot \mathbf{x}}\right],} \\
& {\left[h_{p v}^{+}\left(\mathbf{k}_{3}, \tau_{e}\right), \partial_{k} \partial_{l} h_{i j}^{-}\left(-\mathbf{q}_{3}, \tau\right) e^{i \mathbf{q}_{3} \cdot \mathbf{x}}\right]}
\end{aligned}
$$

plus five more terms that deal with the remaining permutations of the momenta. Similarly, for the second integral we have

$$
\begin{aligned}
& {\left[h_{t m}^{+}\left(\mathbf{k}_{1}, \tau_{e}\right), h_{k l}^{-}\left(-\mathbf{q}_{1}, \tau\right) e^{i \mathbf{q}_{1} \cdot \mathbf{x}}\right], \quad\left[h_{n r}^{+}\left(\mathbf{k}_{2}, \tau_{e}\right), h_{i j}^{-}\left(-\mathbf{q}_{2}, \tau\right) e^{i \mathbf{q}_{2} \cdot \mathbf{x}}\right]} \\
& {\left[h_{p v}^{+}\left(\mathbf{k}_{3}, \tau_{e}\right), \partial_{k} \partial_{l}\left(h_{i j}^{-}\left(-\mathbf{q}_{3}, \tau\right) e^{i \mathbf{q}_{3} \cdot \mathbf{x}}\right)\right]}
\end{aligned}
$$

plus five more terms that deal with the remaining permutations of the momenta. We evaluate the contractions by using (2.3.11) and the notation introduced in (2.3.12). We get 


$$
\begin{aligned}
\left\langle h^{3}\left(\tau_{e}\right)\right\rangle & =2 \operatorname{Re}\left\{-i h\left(k_{1}, \tau_{e}\right) h\left(k_{2}, \tau_{e}\right) h\left(k_{3}, \tau_{e}\right) \int_{-\infty(1-i \epsilon)}^{\tau_{e}} \mathrm{~d} \tau \int \mathrm{d}^{3} x\right. \\
& \times \int \frac{\mathrm{d} q_{1}}{(2 \pi)^{3}} \frac{\mathrm{d} q_{2}}{(2 \pi)^{3}} \frac{\mathrm{d} q_{3}}{(2 \pi)^{3}}\left(-\frac{1}{4} a^{2} \mathcal{F}_{T}\right) \times\left[h^{*}\left(-q_{1}, \tau\right) h^{*}\left(-q_{2}, \tau\right)\right. \\
& \times p_{t m}^{s}\left(\mathbf{k}_{1}\right) p_{j l}^{s *}\left(-\mathbf{q}_{1}\right) p_{n r}^{s}\left(\mathbf{k}_{2}\right) p_{i k}^{s *}\left(-\mathbf{q}_{2}\right)-\frac{1}{2} h^{*}\left(-q_{1}, \tau\right) h^{*}\left(-q_{2}, \tau\right) \\
& \left.\times p_{t m}^{s}\left(\mathbf{k}_{1}\right) p_{k l}^{s *}\left(-\mathbf{q}_{1}\right) p_{n r}^{s}\left(\mathbf{k}_{2}\right) p_{i j}^{s *}\left(-\mathbf{q}_{2}\right)\right] e^{i\left(\mathbf{q}_{1}+\mathbf{q}_{2}\right) \cdot \mathbf{x}} \partial_{k} \partial_{l} h^{*}\left(-q_{3}, \tau\right) \\
& \left.p_{p v}^{s}\left(\mathbf{k}_{3}\right) p_{i j}^{s *}\left(-\mathbf{q}_{3}\right) e^{i \mathbf{q}_{3} \cdot \mathbf{x}} \delta\left(\mathbf{k}_{1}+\mathbf{q}_{1}\right) \delta\left(\mathbf{k}_{2}+\mathbf{q}_{2}\right) \delta\left(\mathbf{k}_{3}+\mathbf{q}_{3}\right)\right\},
\end{aligned}
$$

where we have applied $s=s^{\prime}$ throughout. Next, we integrate over the momenta and use the properties of delta functions to set $\mathbf{k}_{1}=-\mathbf{q}_{1}, \mathbf{k}_{2}=-\mathbf{q}_{2}, \mathbf{k}_{3}=-\mathbf{q}_{3}$. Finally we differentiate the exponential $\left(\partial_{k} \partial_{l} \exp \left[-i \mathbf{k}_{3} \cdot \mathbf{x}\right]=i^{2} \mathbf{k}_{3 l} \cdot \mathbf{k}_{3 k} \exp \left[i \mathbf{k}_{3} \cdot \mathbf{x}\right]\right)$ and integrate over the spatial part of the integral using $\int \mathrm{d}^{3} x \exp \left[-i\left(\mathbf{k}_{1}+\mathbf{k}_{2}+\mathbf{k}_{3}\right) \cdot \mathbf{x}\right]=$ $\delta\left(\mathbf{k}_{1}+\mathbf{k}_{2}+\mathbf{k}_{3}\right)$. We get

$$
\begin{aligned}
\left\langle h^{3}\left(\tau_{e}\right)\right\rangle & =2 \operatorname{Re}\left\{-i h\left(k_{1}, \tau_{e}\right) h\left(k_{2}, \tau_{e}\right) h\left(k_{3}, \tau_{e}\right) \int_{-\infty(1-i \epsilon)}^{\tau_{e}} \mathrm{~d} \tau \frac{1}{4} \frac{f_{t} H_{0}^{6} A_{1}^{2}}{9} \tau^{4}\left(\mathbf{k}_{3 l} \cdot \mathbf{k}_{3 k}\right)\right. \\
& \times\left[h^{*}\left(k_{1}, \tau\right) h^{*}\left(k_{2}, \tau\right) \Pi_{t m, j l}\left(\mathbf{k}_{1}\right) \Pi_{n r, i k}\left(\mathbf{k}_{2}\right)-\frac{1}{2} h^{*}\left(k_{1}, \tau\right) h^{*}\left(k_{2}, \tau\right)\right. \\
& \left.\left.\times \Pi_{t m, k l}\left(\mathbf{k}_{1}\right) \Pi_{n r, i j}\left(\mathbf{k}_{2}\right)\right] h^{*}\left(k_{3}, \tau\right) \Pi_{p v, i j}\left(\mathbf{k}_{3}\right)(2 \pi)^{3} \delta\left(\mathbf{k}_{1}+\mathbf{k}_{2}+\mathbf{k}_{3}\right)\right\},
\end{aligned}
$$

where the minus sign in the brackets was cancelled by the $i^{2}$ factor from the differentiation of the exponential, giving an overall minus sign, and we used the expression for $\mathcal{F}_{T}$ during the second part of the evolution (5.5.24) and the expression for the field velocity (5.5.12), to write

$$
\frac{1}{4} a^{2} \mathcal{F}=a^{2} f t \frac{H_{0}^{2}}{9} A_{1}^{2} a^{-4}=\frac{1}{4} \frac{f_{t} H_{0}^{6} A_{1}^{2}}{9} \tau^{4}
$$

Using the solution in (A.14.7) and its conjugate in (A.14.8), the three point function becomes

$$
\left\langle h^{3}\left(\tau_{e}\right)\right\rangle=(2 \pi)^{3} \delta\left(\mathbf{k}_{1}+\mathbf{k}_{2}+\mathbf{k}_{3}\right) \mathcal{T}_{\text {tmnrpv }}^{(G R)}\left(2 \operatorname{Re}\left[\mathcal{I}^{(G R)}(\tau)\right]\right)
$$

where

$$
\begin{aligned}
\mathcal{T}_{\text {tmnrpv }}^{(G R)} & =\left\{\left(\mathbf{k}_{3 l} \cdot \mathbf{k}_{3 k}\right)\left[\Pi_{t m, j l}\left(\mathbf{k}_{1}\right) \Pi_{n r, i k}\left(\mathbf{k}_{2}\right)-\frac{1}{2} \Pi_{t m, k l}\left(\mathbf{k}_{1}\right) \Pi_{n r, i j}\left(\mathbf{k}_{2}\right)\right]\right. \\
& \left.\times \Pi_{p v, i j}\left(\mathbf{k}_{3}\right)+5 \text { perms of } 1,2,3\right\}
\end{aligned}
$$


and

$$
\begin{aligned}
\mathcal{I}^{(G R)} & =i \frac{y_{0}^{12}}{2^{3}\left(k_{1} k_{2} k_{3}\right)^{3} z_{0}^{6} c_{T}^{18}} \frac{1}{4} \frac{f_{t} H_{0}^{6} A_{1}^{2}}{9} \\
& \times\left(i-c_{T} k_{1} \tau_{e}\right)\left(i-c_{T} k_{2} \tau_{e}\right)\left(i-c_{T} k_{3} \tau_{e}\right) \tau_{e}^{-9} e^{-i c_{T}\left(k_{1}+k_{2}+k_{3}\right) \tau_{0}} \\
& \times \int_{-\infty(1-i \epsilon)}^{\tau_{e}} \mathrm{~d} \tau\left(i+c_{T} k_{1} \tau\right)\left(i+c_{T} k_{2} \tau\right)\left(i+c_{T} k_{3} \tau\right) \tau^{-5} e^{i c_{T}\left(k_{1}+k_{2}+k_{3}\right) \tau}
\end{aligned}
$$

which can be written as

$$
\begin{aligned}
\mathcal{I}^{(G R)} & =i \frac{y_{0}^{12}}{2^{3}\left(k_{1} k_{2} k_{3}\right)^{3} z_{0}^{6} c_{T}^{18}} \frac{1}{4} \frac{f_{t} H_{0}^{6} A_{1}^{2}}{9} \\
& \times\left[-\frac{c_{T}^{3} k_{1} k_{2} k_{3}}{\tau^{6}}+\frac{i c_{T}^{2}\left(k_{1} k_{2}+k_{1} k_{3}+k_{2} k_{3}\right)}{\tau^{7}}+\frac{c_{T} K}{\tau^{8}}-\frac{i}{\tau^{9}}\right] e^{-i c_{T} K \tau_{e}} \\
& \times \int_{-\infty(1-i \epsilon)}^{\tau_{e}} \mathrm{~d} \tau\left[\frac{c_{T}^{3} k_{1} k_{2} k_{3}}{\tau^{2}}+\frac{i c_{T}^{2}\left(k_{1} k_{2}+k_{1} k_{3}+k_{2} k_{3}\right)}{\tau^{3}}-\frac{c_{T} K}{\tau^{4}}\right. \\
& \left.-\frac{i}{\tau^{5}}\right] e^{i c_{T} K \tau}
\end{aligned}
$$

where $K=k_{1}+k_{2}+k_{3}$. The integrals to solve are

$$
\begin{aligned}
I & =\int_{-\infty(1-i \epsilon)}^{\tau_{e}} \mathrm{~d} \tau\left(i+c_{T} k_{1} \tau\right)\left(i+c_{T} k_{2} \tau\right)\left(i+c_{T} k_{3} \tau\right) \tau^{-5} e^{i c_{T}\left(k_{1}+k_{2}+k_{3}\right) \tau} \\
& =\int_{-\infty(1-i \epsilon)}^{\tau_{e}} \mathrm{~d} \tau\left[\frac{c_{T}^{3} k_{1} k_{2} k_{3}}{\tau^{2}}+\frac{i c_{T}^{2}\left(k_{1} k_{2}+k_{1} k_{3}+k_{2} k_{3}\right)}{\tau^{3}}-\frac{c_{T} K}{\tau^{4}}\right. \\
& \left.-\frac{i}{\tau^{5}}\right] e^{i c_{T} K \tau}
\end{aligned}
$$

where we have defined $K=\sum_{i} k_{i}$ and $\left|k_{i}\right|=k_{i}$. We find the following contributions

$$
\begin{aligned}
I_{1} & =\int_{-\infty(1-i \epsilon)}^{\tau_{e}} \mathrm{~d} \tau \frac{c_{T}^{3} k_{1} k_{2} k_{3}}{\tau^{2}} e^{i c_{T} K \tau}=\left.e^{i c_{T} K \tau}\left[-\frac{c_{T}^{3} k_{1} k_{2} k_{3}}{\tau}\right]\right|_{-\infty} ^{\tau_{e}} \\
& +\left.i c_{T}^{4} K k_{1} k_{2} k_{3} E i\left[i c_{T} K \tau\right]\right|_{-\infty} ^{\tau_{e}}, \\
I_{2} & =\int_{-\infty(1-i \epsilon)}^{\tau_{e}} \mathrm{~d} \tau \frac{i c_{T}^{2}\left(k_{1} k_{2}+k_{1} k_{3}+k_{2} k_{3}\right)}{\tau^{3}} e^{i c_{T} K \tau}=e^{i c_{T} K \tau} \\
& \times\left.\left[-\frac{i c_{T}^{2}\left(k_{1} k_{2}+k_{1} k_{3}+k_{2} k_{3}\right)}{2 \tau^{2}}+\frac{c_{T}^{3} K\left(k_{1} k_{2}+k_{1} k_{3}+k_{2} k_{3}\right)}{2 \tau}\right]\right|_{-\infty} ^{\tau_{e}} \\
& -\left.\frac{i}{2} c_{T}^{4} K^{2}\left(k_{1} k_{2}+k_{1} k_{3}+k_{2} k_{3}\right) E i\left[i c_{T} K \tau\right]\right|_{-\infty} ^{\tau_{e}},
\end{aligned}
$$




$$
\begin{aligned}
I_{3} & =-\int_{-\infty(1-i \epsilon)}^{\tau_{e}} \mathrm{~d} \tau \frac{c_{T} K}{\tau^{4}} e^{i c_{T} K \tau}=\left.e^{i c_{T} K \tau}\left[-\frac{c_{T} K}{3 \tau^{3}}-\frac{i c_{T}^{2} K^{2}}{6 \tau^{2}}+\frac{c_{T}^{3} K^{3}}{6 \tau}\right]\right|_{-\infty} ^{\tau_{e}} \\
& -\left.\frac{i}{6} c_{T}^{4} K^{4} E i\left[i c_{T} K \tau\right]\right|_{-\infty} ^{\tau_{e}}, \\
I_{4} & =-\int_{-\infty(1-i \epsilon)}^{\tau_{e}} \mathrm{~d} \tau \frac{i}{\tau^{5}} e^{i c_{T} K \tau}=\left.i e^{i c_{T} K \tau}\left[\frac{1}{\tau^{4}}-\frac{c_{T} K}{12 \tau^{3}}-\frac{c_{T}^{2} K^{2}}{24 \tau^{2}}+\frac{c_{T}^{3} K^{3}}{24 \tau}\right]\right|_{-\infty} ^{\tau_{e}} \\
& -\left.\frac{1}{24} i c_{T}^{4} K^{4} E i\left[i c_{T} K \tau\right]\right|_{-\infty} ^{\tau_{e}},
\end{aligned}
$$

where $E i$ denotes the Exponential Integral of imaginary argument $E i(i x), x \in R$, whose properties are given in Appendix (A.15).

Our results diverge for $\tau_{e} \rightarrow 0$ and they are valid only as long as we are away from the origin $\tau_{e}=0$. From this it follows that, we can express our results for small but non-zero value of the upper limit $t_{e} \ll 1$.

Next we evaluate the integrals. From (A.15.6) we can see that the lower limit of the Exponential Integrals gives constant multiples of $i \pi$, while the rest terms give oscillatory contributions. These can be remedied by employing the $i \epsilon$ prescription so that they decay. Either way, these terms do not concern us, as we are only interested to find the dominant contributions to the bispectrum. We find these by taking the upper limit of the integral for small argument $t_{e} \ll 1$. It can be seen from (A.15.5) that the contributions from the Exponential Integral are also subdominant. Therefore, the leading-order contributions to the integral for small argument $t_{e} \ll 1$, are

$$
\begin{aligned}
I & =\frac{i}{4 \tau_{e}^{4}}+\frac{i c_{T}^{2}\left[K^{2}-2\left(k_{1} k_{2}+k_{1} k_{3}+k_{2} k_{3}\right)\right]}{4 \tau_{e}^{2}} \\
& +\frac{\left.3 c_{T}^{3} K\left(k_{1} k_{2}+k_{1} k_{3}+k_{2} k_{3}\right)-c_{T}^{3} K^{3}-3 c_{T}^{3} k_{1} k_{2} k_{3}\right)}{3 \tau_{e}}+\cdots
\end{aligned}
$$

Similarly we Taylor expand the contributions to the three-point function coming from outside the integral in (A.14.22), for small argument $t_{e} \ll 1$, and keep the leading order-contributions. These are

$$
\begin{aligned}
& =-\frac{i}{\tau_{e}^{9}}-\frac{i c_{T}^{2}\left[K^{2}-2\left(k_{1} k_{2}+k_{1} k_{3}+k_{2} k_{3}\right)\right]}{2 \tau_{e}^{7}} \\
& -\frac{c_{T}^{3}\left[K^{3}+6 k_{1} k_{2} k_{3}-6 K\left(k_{1} k_{2}+k_{1} k_{3}+k_{2} k_{3}\right)\right]}{6 \tau_{e}^{6}}+\cdots
\end{aligned}
$$


Next, we simply multiply (A.14.28) with (A.14.29) and then we multiply the result with the overall imaginary factor in (A.14.22). Finally we take the real part, to find the following leading-order contribution to the three-point function

$$
\operatorname{Re}\left[\mathcal{I}^{(G R)}\right]=\frac{y_{0}^{12}}{2^{3}\left(k_{1} k_{2} k_{3}\right)^{3} z_{0}^{6} c_{T}^{18}} \frac{1}{4} \frac{f_{t} H_{0}^{6} A_{1}^{2}}{9}\left(-\frac{c_{T}^{3}}{4}\left(k_{1}^{3}+k_{2}^{3}+k_{3}^{3}\right) \tau_{e}^{-10}\right) .
$$

In order to compare our results with the literature, we re-express $-\left(k_{1}^{3}+k_{2}^{3}+k_{3}^{3}\right)$, by using the identity for $\left(k_{1}+k_{2}+k_{3}\right)^{3}$. Therefore, we get

$$
\begin{aligned}
\operatorname{Re}\left[\mathcal{I}^{(G R)}\right] & =\frac{y_{0}^{12}}{2^{3}\left(k_{1} k_{2} k_{3}\right)^{3} z_{0}^{6} c_{T}^{18}} \frac{1}{4} \frac{f_{t} H_{0}^{6} A_{1}^{2}}{9} \frac{c_{T}^{3}}{4} \\
& \times \tau_{e}^{-10} K^{3}\left(-1+\frac{3 \sum_{i \neq j} k_{i}^{2} k_{j}}{K^{3}}+\frac{6 \prod_{i} k_{i}}{K^{3}}\right) .
\end{aligned}
$$

Evaluating the constant overall coefficient gives

$$
\frac{y_{0}^{12}}{2^{3} z_{0}^{6} c_{T}^{18}} \frac{1}{4} \frac{f_{t} H_{0}^{6} A_{1}^{2}}{9} \frac{c_{T}^{3}}{4}=\frac{81}{2 f_{t}^{2} A_{1}^{4} H_{0}^{12}}
$$

Therefore, this contribution becomes

$$
\begin{aligned}
\operatorname{Re}\left[\mathcal{I}^{(G R)}\right] & =\frac{81}{2 f_{t}^{2} A_{1}^{4} H_{0}^{12}}\left(\prod_{i} k_{i}\right)^{-3}\left(\mathbf{k}_{3 l} \cdot \mathbf{k}_{3 k}\right) \\
& \times \tau_{e}^{-10} K^{3}\left(-1+\frac{3 \sum_{i \neq j} k_{i}^{2} k_{j}}{K^{3}}+\frac{6 \prod_{i} k_{i}}{K^{3}}\right) .
\end{aligned}
$$

This can be written in terms of the power spectrum squared, per polarization, in the non-attractor era, given in (A.14.12), as

$$
\begin{aligned}
2 \operatorname{Re}\left[\mathcal{I}^{(G R)}\right] & =\frac{c_{T}^{2}}{64} P_{h}^{2}(2 \pi)^{4}\left(\prod_{i} k_{i}\right)^{-3} \\
& \times \tau_{e}^{2} K^{3}\left(-1+\frac{3 \sum_{i \neq j} k_{i}^{2} k_{j}}{K^{3}}+\frac{6 \prod_{i} k_{i}}{K^{3}}\right) .
\end{aligned}
$$

Finally, we define $c_{T} \tau_{e}=y_{\text {end }}$ to get

$$
\begin{aligned}
2 \operatorname{Re}\left[\mathcal{I}^{(G R)}\right] & =\frac{(2 \pi)^{4} P_{h}^{2}}{\prod_{i} k_{i}^{3}} \\
& \times y_{\text {end }}^{2} K^{3}\left(-1+\frac{3 \sum_{i \neq j} k_{i}^{2} k_{j}}{K^{3}}+\frac{6 \prod_{i} k_{i}}{K^{3}}\right) .
\end{aligned}
$$




\section{A.15 The Exponential Integral}

The Exponential Integral of imaginary argument $E i(i x), x \in R$ can be expressed in terms of the real functions $S i(x)$ and $C i(x)$, known as the Sine and Cosine integrals, respectively [273] (page 33 onwards)

$$
\begin{aligned}
& E i(i x)=C_{i}(x)-i\left[\frac{\pi}{2}-S i(x)\right], \quad x>0, \\
& E i(-i x)=C_{i}(x)+i\left[\frac{\pi}{2}-S i(x)\right], \quad x>0,
\end{aligned}
$$

where $S_{i}(-x)=-S_{i}(|x|)$. These have the following convergent series, with $z$ complex,

$$
\begin{aligned}
& S_{i}(z)=\sum_{n=0}^{\infty} \frac{(-1)^{n} z^{2 n+1}}{(2 n+1)(2 n+1) !}=z-\frac{z^{3}}{3 ! 3}+\cdots, \quad|z|<\infty, \\
& C_{i}(z)=\gamma+\ln z+\sum_{n=1}^{\infty} \frac{(-1)^{n} z^{2 n}}{2 n(2 n) !}=\gamma+\log z-\frac{z^{2}}{2 ! 2}+\cdots, \quad|\arg z|<\pi,
\end{aligned}
$$

and limiting behaviour

$$
\begin{aligned}
& S_{i}(\infty)=\frac{\pi}{2}, \quad C_{i}(\infty)=0, \\
& S_{i}(0)=0, \quad C_{i}(+0)=-\infty .
\end{aligned}
$$

\section{$\underline{\text { Asymptotic behaviour for small argument }}$}

Using (A.15.2), the leading order behaviour for small real argument $x$, is

$$
\begin{aligned}
& \lim _{z \rightarrow 0} S_{i}(x) \simeq x \\
& \lim _{z \rightarrow 0} C_{i}(x) \simeq \gamma+\log (x)+i \pi, \quad x>0, \\
& \lim _{z \rightarrow 0} C_{i}(-x) \simeq \gamma+\log (x)-i \pi, \quad x>0 .
\end{aligned}
$$

where $C_{i}(z)$ is determined by analytic continuation (see (3.3.9) in [273]). Therefore, the asymptotic behaviour for the exponential integral for imaginary argument for $x \rightarrow 0$, can be approximated to be

$$
\begin{aligned}
& \lim _{x \rightarrow 0} E i(i x)=\gamma+\log (x)+\frac{i \pi}{2}+i x, \quad x>0, \\
& \lim _{x \rightarrow 0} E i(-i x)=\gamma+\log (x)-\frac{i \pi}{2}-i x, \quad x>0 .
\end{aligned}
$$

\section{$\underline{\text { Asymptotic behaviour for large argument }}$}


Using (3.3.9) in [273]) and the limiting behaviour for $x \rightarrow \infty$ in (A.15.3), we find that (A.15.1) can be approximated to be

$$
\lim _{x \rightarrow \infty} E_{i}(-i x)=-i \pi, \quad x>0
$$




\section{Bibliography}

[1] M. C. Guzzetti, N. Bartolo, M. Liguori, and S. Matarrese, "Gravitational waves from inflation," Riv. Nuovo Cim. 39 no. 9, (2016) 399-495, arXiv:1605.01615 [astro-ph.C0].

[2] Planck Collaboration, Y. Akrami et al., "Planck 2018 results. X. Constraints on inflation," arXiv:1807.06211 [astro-ph.CO].

[3] Planck Collaboration, P. A. R. Ade et al., "Planck 2015 results. XIII. Cosmological parameters," Astron. Astrophys. 594 (2016) A13, arXiv:1502.01589 [astro-ph.CO].

[4] R. Caldwell et al., "Astro2020 Science White Paper: Cosmology with a Space-Based Gravitational Wave Observatory," arXiv: 1903.04657 [astro-ph.CO].

[5] D. Jeong and E. Komatsu, "Primordial non-Gaussianity, scale-dependent bias, and the bispectrum of galaxies," Astrophys. J. 703 (2009) 1230-1248, arXiv:0904.0497 [astro-ph.CO].

[6] T. L. Smith, M. Kamionkowski, and A. Cooray, "Direct detection of the inflationary gravitational wave background," Phys. Rev. D73 (2006) 023504, arXiv:astro-ph/0506422 [astro-ph].

[7] M. Mylova, "Chiral primordial gravitational waves in extended theories of Scalar-Tensor gravity," arXiv:1912.00800 [gr-qc] .

[8] M. Mylova, O. zsoy, S. Parameswaran, G. Tasinato, and I. Zavala, "A new mechanism to enhance primordial tensor fluctuations in single field inflation," JCAP 1812 no. 12, (2018) 024, arXiv:1808.10475 [gr-qc].

[9] O. Ozsoy, M. Mylova, S. Parameswaran, C. Powell, G. Tasinato, and I. Zavala, "Squeezed tensor non-Gaussianity in non-attractor inflation," JCAP 1909 no. 09, (2019) 036, arXiv:1902.04976 [hep-th].

[10] L. Verde, H. Peiris, and R. Jimenez, "Optimizing CMB polarization experiments to constrain inflationary physics," JCAP 0601 (2006) 019, arXiv:astro-ph/0506036 [astro-ph]. 
[11] A. Amblard, A. Cooray, and M. Kaplinghat, "Search for Gravitational Waves in the CMB After WMAP3: Foreground Confusion and The Optimal Frequency Coverage for Foreground Minimization," Phys. Rev. D75 (2007) 083508, arXiv:astro-ph/0610829 [astro-ph].

[12] J. R. Gair, M. Vallisneri, S. L. Larson, and J. G. Baker, "Testing General Relativity with Low-Frequency, Space-Based Gravitational-Wave Detectors," Living Rev. Rel. 16 (2013) 7, arXiv:1212.5575 [gr-qc].

[13] H. Kudoh, A. Taruya, T. Hiramatsu, and Y. Himemoto, "Detecting a gravitational-wave background with next-generation space interferometers," Phys. Rev. D73 (2006) 064006, arXiv:gr-qc/0511145 [gr-qc].

[14] R. Kato and J. Soda, "Probing circular polarization in stochastic gravitational wave background with pulsar timing arrays," Phys. Rev. D93 no. 6, (2016) 062003, arXiv:1512.09139 [gr-qc].

[15] Planck Collaboration, P. A. R. Ade et al., "Planck 2015 results. XX. Constraints on inflation," Astron. Astrophys. 594 (2016) A20, arXiv: 1502.02114 [astro-ph.CO].

[16] S. Dyda, E. E. Flanagan, and M. Kamionkowski, "Vacuum Instability in Chern-Simons Gravity," Phys. Rev. D86 (2012) 124031, arXiv:1208.4871 [gr-qc] .

[17] A. H. Guth, "The Inflationary Universe: A Possible Solution to the Horizon and Flatness Problems," Phys. Rev. D23 (1981) 347-356. [Adv. Ser. Astrophys. Cosmol.3,139(1987)].

[18] A. A. Starobinsky, "A New Type of Isotropic Cosmological Models Without Singularity," Phys. Lett. 91B (1980) 99-102. [,771(1980)].

[19] A. D. Linde, "A New Inflationary Universe Scenario: A Possible Solution of the Horizon, Flatness, Homogeneity, Isotropy and Primordial Monopole Problems," Phys. Lett. 108B (1982) 389-393. [Adv. Ser. Astrophys. Cosmol.3,149(1987)].

[20] A. Albrecht and P. J. Steinhardt, "Cosmology for Grand Unified Theories with Radiatively Induced Symmetry Breaking," Phys. Rev. Lett. 48 (1982) 1220-1223. [Adv. Ser. Astrophys. Cosmol.3,158(1987)].

[21] A. R. Liddle, "An Introduction to cosmological inflation," in Proceedings, Summer School in High-energy physics and cosmology: Trieste, Italy, June 29-July 17, 1998, pp. 260-295. 1999. arXiv:astro-ph/9901124 [astro-ph].

[22] V. F. Mukhanov and G. V. Chibisov, "Quantum Fluctuations and a Nonsingular Universe," JETP Lett. 33 (1981) 532-535. [Pisma Zh. Eksp. Teor. Fiz.33,549(1981)]. 
[23] A. H. Guth and S. Y. Pi, "Fluctuations in the New Inflationary Universe," Phys. Rev. Lett. 49 (1982) 1110-1113.

[24] S. M. Carroll, Spacetime and Geometry. Cambridge University Press, 2019.

[25] R. M. Wald, General Relativity. Chicago Univ. Pr., Chicago, USA, 1984.

[26] S. Weinberg, Cosmology. OUP Oxford, 2008.

[27] M. P. Hobson, G. P. Efstathiou, and A. N. Lasenby, General relativity: An introduction for physicists. 2006.

[28] C. W. Misner, K. S. Thorne, and J. A. Wheeler, Gravitation. W. H. Freeman, San Francisco, 1973.

[29] E. Schrödinger, Expanding Universe. Cambridge University Press, 2011.

[30] A. R. Liddle, An introduction to modern cosmology. 1998.

[31] A. R. Liddle and D. H. Lyth, Cosmological inflation and large scale structure. 2000.

[32] P. Coles, G. Ellis, C. Peter, G. Ellis, G. Ellis, C. U. Press, P. Goddard, and J. Yeomans, Is the Universe Open Or Closed?: The Density of Matter in the Universe. Cambridge Lecture Notes in Physics. Cambridge University Press, 1997.

[33] Planck Collaboration, N. Aghanim et al., "Planck 2018 results. VI. Cosmological parameters," arXiv:1807.06209 [astro-ph.CO].

[34] W. H. Kinney, "Tasi lectures on inflation," 2009.

[35] A. Riotto, "Inflation and the theory of cosmological perturbations," ICTP Lect. Notes Ser. 14 (2003) 317-413, arXiv:hep-ph/0210162 [hep-ph].

[36] T. Kobayashi, M. Yamaguchi, and J. Yokoyama, "Generalized G-inflation: Inflation with the most general second-order field equations," Prog. Theor. Phys. 126 (2011) 511-529, arXiv:1105.5723 [hep-th].

[37] D. Baumann, "Inflation," in Physics of the large and the small, TASI 09, proceedings of the Theoretical Advanced Study Institute in Elementary Particle Physics, Boulder, Colorado, USA, 1-26 June 2009, pp. 523-686. 2011. arXiv:0907.5424 [hep-th].

[38] A. Riotto, "Inflation and the theory of cosmological perturbations," ICTP Lect. Notes Ser. 14 (2003) 317-413, arXiv:hep-ph/0210162 [hep-ph].

[39] J. M. Bardeen, "Gauge Invariant Cosmological Perturbations," Phys. Rev. D22 (1980) 1882-1905. 
[40] D. Baumann, L. Senatore, and M. Zaldarriaga, "Scale-Invariance and the Strong Coupling Problem," JCAP 1105 (2011) 004, arXiv:1101.3320 [hep-th].

[41] N. D. Birrell and P. C. W. Davies, Quantum Fields in Curved Space. Cambridge Monographs on Mathematical Physics. Cambridge Univ. Press, Cambridge, UK, 1984.

[42] S. Weinberg, "Quantum contributions to cosmological correlations," Phys. Rev. D72 (2005) 043514, arXiv:hep-th/0506236 [hep-th].

[43] X. Gao, T. Kobayashi, M. Yamaguchi, and J. Yokoyama, "Primordial non-Gaussianities of gravitational waves in the most general single-field inflation model," Phys. Rev. Lett. 107 (2011) 211301, arXiv:1108.3513 [astro-ph.CO].

[44] A. A. Starobinsky, "Spectrum of relict gravitational radiation and the early state of the universe," JETP Lett. 30 (1979) 682-685. [,767(1979)].

[45] V. A. Rubakov, M. V. Sazhin, and A. V. Veryaskin, "Graviton Creation in the Inflationary Universe and the Grand Unification Scale," Phys. Lett. 115B (1982) 189-192.

[46] M. Kamionkowski, A. Kosowsky, and A. Stebbins, "A Probe of primordial gravity waves and vorticity," Phys. Rev. Lett. 78 (1997) 2058-2061, arXiv:astro-ph/9609132 [astro-ph].

[47] U. Seljak and M. Zaldarriaga, "Signature of gravity waves in polarization of the microwave background," Phys. Rev. Lett. 78 (1997) 2054-2057, arXiv:astro-ph/9609169 [astro-ph].

[48] WMAP Collaboration, C. L. Bennett et al., "Nine-Year Wilkinson Microwave Anisotropy Probe (WMAP) Observations: Final Maps and Results," Astrophys. J. Suppl. 208 (2013) 20, arXiv:1212.5225 [astro-ph.CO].

[49] COBE Collaboration, G. F. Smoot et al., "Structure in the COBE differential microwave radiometer first year maps," Astrophys. J. 396 (1992) L1-L5.

[50] E. M. Leitch, J. M. Kovac, C. Pryke, B. Reddall, E. S. Sandberg, M. Dragovan, J. E. Carlstrom, N. W. Halverson, and W. L. Holzapfel, "Measuring polarization with DASI," Nature 420 (2002) 763-771, arXiv:astro-ph/0209476 [astro-ph].

[51] M. Kamionkowski and A. Kosowsky, "Detectability of inflationary gravitational waves with microwave background polarization," Phys. Rev. D57 (1998) 685-691, arXiv:astro-ph/9705219 [astro-ph]. 
[52] W. Hu and T. Okamoto, "Mass reconstruction with cmb polarization," Astrophys. J. 574 (2002) 566-574, arXiv:astro-ph/0111606 [astro-ph].

[53] A. Lewis and A. Challinor, "Weak gravitational lensing of the CMB," Phys. Rept. 429 (2006) 1-65, arXiv:astro-ph/0601594 [astro-ph].

[54] BICEP2 Collaboration, P. A. R. Ade et al., "Detection of B-Mode Polarization at Degree Angular Scales by BICEP2," Phys. Rev. Lett. 112 no. 24, (2014) 241101, arXiv:1403.3985 [astro-ph.CO].

[55] BICEP2 Collaboration, P. A. R. Ade et al., "BICEP2 II: Experiment and Three-Year Data Set," Astrophys. J. 792 no. 1, (2014) 62, arXiv:1403.4302 [astro-ph.CO].

[56] BICEP2, Planck Collaboration, P. A. R. Ade et al., "Joint Analysis of BICEP2/KeckArray and Planck Data," Phys. Rev. Lett. 114 (2015) 101301, arXiv:1502.00612 [astro-ph.CO].

[57] POLARBEAR Collaboration, P. A. R. Ade et al., "A Measurement of the Cosmic Microwave Background B-Mode Polarization Power Spectrum at Sub-Degree Scales with POLARBEAR," Astrophys. J. 794 no. 2, (2014) 171, arXiv: 1403.2369 [astro-ph.CO].

[58] E. Calabrese et al., "Precision Epoch of Reionization studies with next-generation CMB experiments," JCAP 1408 (2014) 010, arXiv:1406.4794 [astro-ph.CO].

[59] J. R. Eimer, C. L. Bennett, D. T. Chuss, T. A. Marriage, E. J. Wollack, and L. Zeng, "The Cosmology Large Angular Scale Surveyor (CLASS): $40 \mathrm{GHz}$ optical design," Proc. SPIE Int. Soc. Opt. Eng. 8452 (2012) 845220, arXiv:1211.0041 [astro-ph.IM].

[60] B. P. Crill et al., "SPIDER: A Balloon-borne Large-scale CMB Polarimeter," Proc. SPIE Int. Soc. Opt. Eng. 7010 (2008) 70102P, arXiv:0807.1548 [astro-ph].

[61] T. Matsumura et al., "Mission design of LiteBIRD," arXiv:1311.2847 [astro-ph. IM]. [J. Low. Temp. Phys.176,733(2014)].

[62] PRISM Collaboration, P. Andr et al., "PRISM (Polarized Radiation Imaging and Spectroscopy Mission): An Extended White Paper," JCAP 1402 (2014) 006, arXiv:1310.1554 [astro-ph.CO].

[63] E. Komatsu, N. Afshordi, N. Bartolo, D. Baumann, J. R. Bond, E. I. Buchbinder, C. T. Byrnes, X. Chen, D. J. H. Chung, A. Cooray, P. Creminelli, N. Dalal, O. Dore, R. Easther, A. V. Frolov, K. M. Gorski, M. G. Jackson, J. Khoury, W. H. Kinney, L. Kofman, K. Koyama, L. Leblond, J. L. Lehners, J. E. Lidsey, M. Liguori, E. A. Lim, A. Linde, D. H. Lyth, J. Maldacena, S. Matarrese, L. McAllister, P. McDonald, 
S. Mukohyama, B. Ovrut, H. V. Peiris, C. Raeth, A. Riotto, Y. Rodriguez, M. Sasaki, R. Scoccimarro, D. Seery, E. Sefusatti, U. Seljak, L. Senatore, S. Shandera, E. P. S. Shellard, E. Silverstein, A. Slosar, K. M. Smith, A. A. Starobinsky, P. J. Steinhardt, F. Takahashi, M. Tegmark, A. J. Tolley, L. Verde, B. D. Wandelt, D. Wands, S. Weinberg, M. Wyman, A. P. S. Yadav, and M. Zaldarriaga, "Non-gaussianity as a probe of the physics of the primordial universe and the astrophysics of the low redshift universe," 2009.

[64] J. M. Maldacena, "Non-Gaussian features of primordial fluctuations in single field inflationary models," JHEP 05 (2003) 013, arXiv : astro-ph/0210603 [astro-ph].

[65] H. Collins, "Primordial non-Gaussianities from inflation," arXiv:1101.1308 [astro-ph.CO].

[66] E. Komatsu and D. N. Spergel, "Acoustic signatures in the primary microwave background bispectrum," Phys. Rev. D63 (2001) 063002, arXiv:astro-ph/0005036 [astro-ph].

[67] C. T. Byrnes, "Lecture notes on non-Gaussianity," Astrophys. Space Sci. Proc. 45 (2016) 135-165, arXiv:1411.7002 [astro-ph.C0].

[68] A. Gangui, F. Lucchin, S. Matarrese, and S. Mollerach, "The Three point correlation function of the cosmic microwave background in inflationary models," Astrophys. J. 430 (1994) 447-457, arXiv:astro-ph/9312033 [astro-ph].

[69] P. Creminelli and M. Zaldarriaga, "Single field consistency relation for the 3-point function," JCAP 0410 (2004) 006, arXiv: astro-ph/0407059 [astro-ph].

[70] M. Alishahiha, E. Silverstein, and D. Tong, "DBI in the sky," Phys. Rev. D70 (2004) 123505, arXiv:hep-th/0404084 [hep-th].

[71] C. Burrage, C. de Rham, D. Seery, and A. J. Tolley, "Galileon inflation," JCAP 1101 (2011) 014, arXiv:1009.2497 [hep-th].

[72] Planck Collaboration, Y. Akrami et al., "Planck 2018 results. IX. Constraints on primordial non-Gaussianity," arXiv: 1905.05697 [astro-ph.CO].

[73] Planck Collaboration, P. A. R. Ade et al., "Planck 2015 results. XVII. Constraints on primordial non-Gaussianity," Astron. Astrophys. 594 (2016) A17, arXiv:1502.01592 [astro-ph.C0].

[74] N. Bartolo, E. Komatsu, S. Matarrese, and A. Riotto, "Non-Gaussianity from inflation: Theory and observations," Phys. Rept. 402 (2004) 103-266, arXiv:astro-ph/0406398 [astro-ph]. 
[75] X. Chen, "Primordial Non-Gaussianities from Inflation Models," Adv. Astron. 2010 (2010) 638979, arXiv:1002.1416 [astro-ph.CO].

[76] Y. Wang, "Inflation, Cosmic Perturbations and Non-Gaussianities," Commun. Theor. Phys. 62 (2014) 109-166, arXiv:1303.1523 [hep-th].

[77] J. F. Donoghue, "Introduction to the effective field theory description of gravity," in Advanced School on Effective Theories Almunecar, Spain, June 25-July 1, 1995. 1995. arXiv:gr-qc/9512024 [gr-qc].

[78] C. P. Burgess, "Quantum gravity in everyday life: General relativity as an effective field theory," Living Rev. Rel. 7 (2004) 5-56, arXiv:gr-qc/0311082 [gr-qc].

[79] S. Weinberg, Cosmology. OUP Oxford, 2008.

[80] S. Weinberg, "Effective Field Theory for Inflation," Phys. Rev. D77 (2008) 123541, arXiv:0804.4291 [hep-th].

[81] A. Petrov and A. Blechman, Effective Field Theories. World Scientific Publishing Company Pte Limited, 2015.

[82] S. Weinberg, "Phenomenological Lagrangians," Physica A96 no. 1-2, (1979) 327-340.

[83] D. Lovelock, "The Einstein tensor and its generalizations," J. Math. Phys. 12 (1971) 498-501.

[84] D. Lovelock, "The four-dimensionality of space and the einstein tensor," $J$. Math. Phys. 13 (1972) 874-876.

[85] A. Nicolis, R. Rattazzi, and E. Trincherini, "The Galileon as a local modification of gravity," Phys. Rev. D79 (2009) 064036, arXiv:0811.2197 [hep-th].

[86] C. Deffayet, G. Esposito-Farese, and A. Vikman, "Covariant Galileon," Phys. Rev. D79 (2009) 084003, arXiv:0901.1314 [hep-th].

[87] C. Deffayet, X. Gao, D. A. Steer, and G. Zahariade, "From k-essence to generalised Galileons," Phys. Rev. D84 (2011) 064039, arXiv:1103.3260 [hep-th].

[88] G. W. Horndeski, "Second-order scalar-tensor field equations in a four-dimensional space," Int. J. Theor. Phys. 10 (1974) 363-384.

[89] A. Linde, M. Noorbala, and A. Westphal, "Observational consequences of chaotic inflation with nonminimal coupling to gravity," JCAP 1103 (2011) 013, arXiv:1101.2652 [hep-th].

[90] C. Armendariz-Picon, T. Damour, and V. F. Mukhanov, "k - inflation," Phys. Lett. B458 (1999) 209-218, arXiv:hep-th/9904075 [hep-th]. 
[91] J. Garriga and V. F. Mukhanov, "Perturbations in k-inflation," Phys. Lett. B458 (1999) 219-225, arXiv:hep-th/9904176 [hep-th].

[92] E. Silverstein and D. Tong, "Scalar speed limits and cosmology: Acceleration from D-cceleration," Phys. Rev. D70 (2004) 103505, arXiv:hep-th/0310221 [hep-th].

[93] R. L. Arnowitt, S. Deser, and C. W. Misner, "The Dynamics of general relativity," Gen. Rel. Grav. 40 (2008) 1997-2027, arXiv:gr-qc/0405109 $[\mathrm{gr}-\mathrm{qc}]$.

[94] T. Kobayashi, "Horndeski theory and beyond: a review," Rept. Prog. Phys. 82 no. 8, (2019) 086901, arXiv:1901.07183 [gr-qc].

[95] T. Clifton, P. G. Ferreira, A. Padilla, and C. Skordis, "Modified Gravity and Cosmology," Phys. Rept. 513 (2012) 1-189, arXiv:1106. 2476 [astro-ph.CO].

[96] A. A. Tseytlin, "Born-Infeld action, supersymmetry and string theory," arXiv:hep-th/9908105 [hep-th].

[97] R. Jackiw and S. Y. Pi, "Chern-Simons modification of general relativity," Phys. Rev. D68 (2003) 104012, arXiv:gr-qc/0308071 [gr-qc].

[98] A. Lue, L.-M. Wang, and M. Kamionkowski, "Cosmological signature of new parity violating interactions," Phys. Rev. Lett. 83 (1999) 1506-1509, arXiv:astro-ph/9812088 [astro-ph].

[99] S. Saito, K. Ichiki, and A. Taruya, "Probing polarization states of primordial gravitational waves with CMB anisotropies," JCAP 0709 (2007) 002, arXiv:0705.3701 [astro-ph].

[100] N. Seto, "Prospects for direct detection of circular polarization of gravitational-wave background," Phys. Rev. Lett. 97 (2006) 151101, arXiv:astro-ph/0609504 [astro-ph].

[101] N. Seto and A. Taruya, "Measuring a Parity Violation Signature in the Early Universe via Ground-based Laser Interferometers," Phys. Rev. Lett. 99 (2007) 121101, arXiv:0707.0535 [astro-ph].

[102] CMBPol Study Team Collaboration, D. Baumann et al., "CMBPol Mission Concept Study: Probing Inflation with CMB Polarization," AIP Conf. Proc. 1141 no. 1, (2009) 10-120, arXiv:0811.3919 [astro-ph].

[103] M. Hazumi et al., "LiteBIRD: A Satellite for the Studies of B-Mode Polarization and Inflation from Cosmic Background Radiation Detection," J. Low. Temp. Phys. 194 no. 5-6, (2019) 443-452. 
[104] S. G. Crowder, R. Namba, V. Mandic, S. Mukohyama, and M. Peloso, "Measurement of Parity Violation in the Early Universe using Gravitational-wave Detectors," Phys. Lett. B726 (2013) 66-71, arXiv:1212.4165 [astro-ph.CO].

[105] T. L. Smith and R. Caldwell, "Sensitivity to a Frequency-Dependent Circular Polarization in an Isotropic Stochastic Gravitational Wave Background," Phys. Rev. D95 no. 4, (2017) 044036, arXiv:1609.05901 [gr-qc].

[106] V. Domcke, J. Garcia-Bellido, M. Peloso, M. Pieroni, A. Ricciardone, L. Sorbo, and G. Tasinato, "Measuring the net circular polarization of the stochastic gravitational wave background with interferometers," arXiv:1910.08052 [astro-ph.CO].

[107] V. Gluscevic and M. Kamionkowski, "Testing parity-violating mechanisms with cosmic microwave background experiments," Phys. Rev. D 81 (Jun, 2010) 123529.

[108] F. Nati, M. J. Devlin, M. Gerbino, B. R. Johnson, B. Keating, L. Pagano, and G. Teply, "POLOCALC: a Novel Method to Measure the Absolute Polarization Orientation of the Cosmic Microwave Background," J. Astron. Inst. 06 no. 02, (2017) 1740008, arXiv:1704.02704 [astro-ph.IM].

[109] S. Shandera et al., "Probing the origin of our Universe through cosmic microwave background constraints on gravitational waves," arXiv:1903.04700 [astro-ph.CO].

[110] S. Alexander and J. Martin, "Birefringent gravitational waves and the consistency check of inflation," Phys. Rev. D71 (2005) 063526, arXiv:hep-th/0410230 [hep-th].

[111] M. Satoh, S. Kanno, and J. Soda, "Circular Polarization of Primordial Gravitational Waves in String-inspired Inflationary Cosmology," Phys. Rev. D77 (2008) 023526, arXiv:0706.3585 [astro-ph].

[112] M. Satoh and J. Soda, "Higher Curvature Corrections to Primordial Fluctuations in Slow-roll Inflation," JCAP 0809 (2008) 019, arXiv:0806.4594 [astro-ph].

[113] M. Satoh, "Slow-roll Inflation with the Gauss-Bonnet and Chern-Simons Corrections," JCAP 1011 (2010) 024, arXiv: 1008.2724 [astro-ph.CO].

[114] L. Sorbo, "Parity violation in the Cosmic Microwave Background from a pseudoscalar inflaton," JCAP 1106 (2011) 003, arXiv:1101.1525 [astro-ph.CO].

[115] Y. Cai, Y.-T. Wang, and Y.-S. Piao, "Chirality oscillation of primordial gravitational waves during inflation," JHEP 03 (2017) 024, arXiv:1608.06508 [astro-ph.C0]. 
[116] A. Maleknejad, M. Noorbala, and M. M. Sheikh-Jabbari, "Leptogenesis in inflationary models with non-Abelian gauge fields," Gen. Rel. Grav. 50 no. 9, (2018) 110, arXiv:1208.2807 [hep-th].

[117] S. H.-S. Alexander, M. E. Peskin, and M. M. Sheikh-Jabbari, "Leptogenesis from gravity waves in models of inflation," Phys. Rev. Lett. 96 (2006) 081301, arXiv:hep-th/0403069 [hep-th].

[118] S. Alexander, E. McDonough, and D. N. Spergel, "Chiral Gravitational Waves and Baryon Superfluid Dark Matter," JCAP 1805 no. 05, (2018) 003, arXiv:1801.07255 [hep-th].

[119] T. Takahashi and J. Soda, "Chiral Primordial Gravitational Waves from a Lifshitz Point," Phys. Rev. Lett. 102 (2009) 231301, arXiv:0904.0554 [hep-th].

[120] A. Wang, Q. Wu, W. Zhao, and T. Zhu, "Polarizing primordial gravitational waves by parity violation," Phys. Rev. D87 no. 10, (2013) 103512, arXiv:1208.5490 [astro-ph.CO].

[121] E. McDonough and S. Alexander, "Observable Chiral Gravitational Waves from Inflation in String Theory," JCAP 1811 no. 11, (2018) 030, arXiv:1806.05684 [hep-th].

[122] C. Cheung, P. Creminelli, A. L. Fitzpatrick, J. Kaplan, and L. Senatore, "The Effective Field Theory of Inflation," JHEP 03 (2008) 014, arXiv:0709.0293 [hep-th].

[123] D. Baumann and D. Green, "Equilateral Non-Gaussianity and New Physics on the Horizon," JCAP 1109 (2011) 014, arXiv:1102.5343 [hep-th].

[124] R. Gwyn, G. A. Palma, M. Sakellariadou, and S. Sypsas, "Effective field theory of weakly coupled inflationary models," JCAP 1304 (2013) 004, arXiv:1210.3020 [hep-th].

[125] C. de Rham and S. Melville, "Unitary null energy condition violation in P(X) cosmologies," Phys. Rev. D95 no. 12, (2017) 123523, arXiv:1703.00025 [hep-th].

[126] A. R. Solomon and M. Trodden, "Higher-derivative operators and effective field theory for general scalar-tensor theories," JCAP 1802 no. 02, (2018) 031, arXiv:1709.09695 [hep-th].

[127] C. de Rham and S. Melville, "Gravitational Rainbows: LIGO and Dark Energy at its Cutoff," Phys. Rev. Lett. 121 no. 22, (2018) 221101, arXiv:1806.09417 [hep-th].

[128] M. Crisostomi, K. Noui, C. Charmousis, and D. Langlois, "Beyond Lovelock gravity: Higher derivative metric theories," Phys. Rev. D97 no. 4, (2018) 044034, arXiv:1710.04531 [hep-th]. 
[129] D. Bettoni and S. Liberati, "Disformal invariance of second order scalar-tensor theories: Framing the Horndeski action," Phys. Rev. D88 (2013) 084020, arXiv:1306.6724 [gr-qc].

[130] M. Zumalacrregui and J. Garca-Bellido, "Transforming gravity: from derivative couplings to matter to second-order scalar-tensor theories beyond the Horndeski Lagrangian," Phys. Rev. D89 (2014) 064046, arXiv:1308.4685 [gr-qc].

[131] M. Crisostomi, K. Koyama, and G. Tasinato, "Extended Scalar-Tensor Theories of Gravity," JCAP 1604 no. 04, (2016) 044, arXiv: 1602.03119 [hep-th].

[132] J. Ben Achour, D. Langlois, and K. Noui, "Degenerate higher order scalar-tensor theories beyond Horndeski and disformal transformations," Phys. Rev. D93 no. 12, (2016) 124005, arXiv:1602.08398 [gr-qc].

[133] G. Domnech, A. Naruko, and M. Sasaki, "Cosmological disformal invariance," JCAP 1510 no. 10, (2015) 067, arXiv:1505.00174 [gr-qc].

[134] D. Baumann and L. McAllister, Inflation and String Theory. CAMBRIDGE MONOGRAPHS ON MATHEM. Cambridge University Press, 2015.

[135] P. Creminelli, J. Gleyzes, J. Norea, and F. Vernizzi, "Resilience of the standard predictions for primordial tensor modes," Phys. Rev. Lett. 113 no. 23, (2014) 231301, arXiv:1407.8439 [astro-ph.Co].

[136] D. Baumann, H. Lee, and G. L. Pimentel, "High-Scale Inflation and the Tensor Tilt," JHEP 01 (2016) 101, arXiv:1507.07250 [hep-th].

[137] J. Soda, H. Kodama, and M. Nozawa, "Parity Violation in Graviton Non-gaussianity," JHEP 08 (2011) 067, arXiv:1106.3228 [hep-th].

[138] C. Pitrou, X. Roy, and O. Umeh, "xPand: An algorithm for perturbing homogeneous cosmologies," Class. Quant. Grav. 30 (2013) 165002, arXiv:1302.6174 [astro-ph.CO].

[139] C. Armendariz-Picon, M. Fontanini, R. Penco, and M. Trodden, "Where does Cosmological Perturbation Theory Break Down?," Class. Quant. Grav. 26 (2009) 185002, arXiv:0805.0114 [hep-th].

[140] J. Qiao, T. Zhu, W. Zhao, and A. Wang, "Polarized primordial gravitational waves in the ghost-free parity-violating gravity," arXiv:1911.01580 [astro-ph.CO].

[141] J. M. Maldacena and G. L. Pimentel, "On graviton non-Gaussianities during inflation," JHEP 09 (2011) 045, arXiv:1104.2846 [hep-th]. 
[142] N. Bartolo and G. Orlando, "Parity breaking signatures from a Chern-Simons coupling during inflation: the case of non-Gaussian gravitational waves," JCAP 1707 (2017) 034, arXiv:1706.04627 [astro-ph.C0].

[143] M. Ostrogradsky, "Mmoires sur les quations diffrentielles, relatives au problme des isoprimtres," Mem. Acad. St. Petersbourg 6 no. 4, (1850) $385-517$.

[144] R. P. Woodard, "Ostrogradsky's theorem on Hamiltonian instability," Scholarpedia 10 no. 8, (2015) 32243, arXiv:1506.02210 [hep-th].

[145] J.-O. Gong, M.-S. Seo, and S. Sypsas, "Higher derivatives and power spectrum in effective single field inflation," JCAP 1503 no. 03, (2015) 009, arXiv:1407.8268 [hep-th].

[146] H. Georgi, "On-shell effective field theory," Nucl. Phys. B361 (1991) 339-350.

[147] C. Grosse-Knetter, "Effective Lagrangians with higher derivatives and equations of motion," Phys. Rev. D49 (1994) 6709-6719, arXiv:hep-ph/9306321 [hep-ph].

[148] C. Arzt, "Reduced effective Lagrangians," Phys. Lett. B342 (1995) 189-195, arXiv:hep-ph/9304230 [hep-ph].

[149] A. V. Manohar, "Introduction to Effective Field Theories," in Les Houches summer school: EFT in Particle Physics and Cosmology Les Houches, Chamonix Valley, France, July 3-28, 2017. 2018. arXiv:1804.05863 [hep-ph].

[150] C. Cheung and G. N. Remmen, "Positivity of Curvature-Squared Corrections in Gravity," Phys. Rev. Lett. 118 no. 5, (2017) 051601, arXiv:1608.02942 [hep-th].

[151] J. Martin and D. J. Schwarz, "New exact solutions for inflationary cosmological perturbations," Phys. Lett. B500 (2001) 1-7, arXiv:astro-ph/0005542 [astro-ph].

[152] L. Bordin, G. Cabass, P. Creminelli, and F. Vernizzi, "Simplifying the EFT of Inflation: generalized disformal transformations and redundant couplings," JCAP 1709 no. 09, (2017) 043, arXiv:1706.03758 [astro-ph.Co].

[153] T. P. Sotiriou, V. Faraoni, and S. Liberati, "Theory of gravitation theories: A No-progress report," Int. J. Mod. Phys. D17 (2008) 399-423, arXiv:0707.2748 [gr-qc].

[154] J. Chagoya and G. Tasinato, "A geometrical approach to degenerate scalar-tensor theories," JHEP 02 (2017) 113, arXiv:1610.07980 [hep-th]. 
[155] V. F. Mukhanov, H. A. Feldman, and R. H. Brandenberger, "Theory of cosmological perturbations. Part 1. Classical perturbations. Part 2. Quantum theory of perturbations. Part 3. Extensions," Phys. Rept. 215 (1992) 203-333.

[156] S. Inoue and J. Yokoyama, "Curvature perturbation at the local extremum of the inflaton's potential," Phys. Lett. B524 (2002) 15-20, arXiv:hep-ph/0104083 [hep-ph].

[157] A. D. Linde, "Fast roll inflation," JHEP 11 (2001) 052, arXiv:hep-th/0110195 [hep-th].

[158] W. H. Kinney, "Horizon crossing and inflation with large eta," Phys. Rev. D72 (2005) 023515, arXiv:gr-qc/0503017 [gr-qc].

[159] J. Martin, H. Motohashi, and T. Suyama, "Ultra Slow-Roll Inflation and the non-Gaussianity Consistency Relation," Phys. Rev. D87 no. 2, (2013) 023514, arXiv:1211.0083 [astro-ph.CO].

[160] H. Motohashi, A. A. Starobinsky, and J. Yokoyama, "Inflation with a constant rate of roll," JCAP 1509 (2015) 018, arXiv:1411.5021 [astro-ph.CO].

[161] Z. Yi and Y. Gong, "On the constant-roll inflation," JCAP 1803 no. 03, (2018) 052, arXiv:1712.07478 [gr-qc].

[162] K. Dimopoulos, "Ultra slow-roll inflation demystified," Phys. Lett. B775 (2017) 262-265, arXiv:1707.05644 [hep-ph].

[163] C. Pattison, V. Vennin, H. Assadullahi, and D. Wands, "The attractive behaviour of ultra-slow-roll inflation," JCAP 1808 no. 08, (2018) 048, arXiv:1806.09553 [astro-ph.CO].

[164] A. A. Starobinsky, "Spectrum of adiabatic perturbations in the universe when there are singularities in the inflation potential," JETP Lett. 55 (1992) 489-494. [Pisma Zh. Eksp. Teor. Fiz.55,477(1992)].

[165] J. Garcia-Bellido, "Massive Primordial Black Holes as Dark Matter and their detection with Gravitational Waves," J. Phys. Conf. Ser. 840 no. 1, (2017) 012032, arXiv:1702.08275 [astro-ph.CO].

[166] M. Sasaki, T. Suyama, T. Tanaka, and S. Yokoyama, "Primordial black holes?perspectives in gravitational wave astronomy," Class. Quant. Grav. 35 no. 6, (2018) 063001, arXiv:1801.05235 [astro-ph.CO].

[167] B. Carr, F. Kuhnel, and M. Sandstad, "Primordial Black Holes as Dark Matter," Phys. Rev. D94 no. 8, (2016) 083504, arXiv:1607.06077 [astro-ph.CO]. 
[168] J. Garcia-Bellido and E. Ruiz Morales, "Primordial black holes from single field models of inflation," Phys. Dark Univ. 18 (2017) 47-54, arXiv:1702.03901 [astro-ph.CO].

[169] C. Germani and T. Prokopec, "On primordial black holes from an inflection point," Phys. Dark Univ. 18 (2017) 6-10, arXiv:1706.04226 [astro-ph.CO].

[170] H. Motohashi and W. Hu, "Primordial Black Holes and Slow-Roll Violation," Phys. Rev. D96 no. 6, (2017) 063503, arXiv:1706.06784 [astro-ph.CO].

[171] G. Ballesteros and M. Taoso, "Primordial black hole dark matter from single field inflation," Phys. Rev. D97 no. 2, (2018) 023501, arXiv:1709.05565 [hep-ph].

[172] J. M. Ezquiaga, J. Garcia-Bellido, and E. Ruiz Morales, "Primordial Black Hole production in Critical Higgs Inflation," Phys. Lett. B776 (2018) 345-349, arXiv:1705.04861 [astro-ph.CO].

[173] M. Cicoli, V. A. Diaz, and F. G. Pedro, "Primordial Black Holes from String Inflation," JCAP 1806 no. 06, (2018) 034, arXiv:1803.02837 [hep-th].

[174] O. zsoy, S. Parameswaran, G. Tasinato, and I. Zavala, "Mechanisms for Primordial Black Hole Production in String Theory," JCAP 1807 (2018) 005, arXiv:1803.07626 [hep-th].

[175] M. Biagetti, G. Franciolini, A. Kehagias, and A. Riotto, "Primordial Black Holes from Inflation and Quantum Diffusion," JCAP 1807 no. 07, (2018) 032, arXiv:1804.07124 [astro-ph.CO].

[176] R. Saito, J. Yokoyama, and R. Nagata, "Single-field inflation, anomalous enhancement of superhorizon fluctuations, and non-Gaussianity in primordial black hole formation," JCAP 0806 (2008) 024, arXiv:0804.3470 [astro-ph].

[177] K. Kannike, L. Marzola, M. Raidal, and H. Veerme, "Single Field Double Inflation and Primordial Black Holes," JCAP 1709 no. 09, (2017) 020, arXiv:1705.06225 [astro-ph.CO].

[178] D. Wands, "Duality invariance of cosmological perturbation spectra," Phys. Rev. D60 (1999) 023507, arXiv:gr-qc/9809062 [gr-qc].

[179] M. Maggiore, Gravitational Waves. Vol. 1: Theory and Experiments. Oxford Master Series in Physics. Oxford University Press, 2007. http://www . oup.com/uk/catalogue/?ci=9780198570745.

[180] M. Maggiore, Gravitational Waves. Vol. 2: Astrophysics and Cosmology. Oxford University Press, 2018. 
[181] S. Chongchitnan and G. Efstathiou, "Prospects for direct detection of primordial gravitational waves," Phys. Rev. D73 (2006) 083511, arXiv:astro-ph/0602594 [astro-ph].

[182] M. Kamionkowski and E. D. Kovetz, "The Quest for B Modes from Inflationary Gravitational Waves," Ann. Rev. Astron. Astrophys. 54 (2016) 227-269, arXiv:1510.06042 [astro-ph.CO].

[183] J. L. Cook and L. Sorbo, "Particle production during inflation and gravitational waves detectable by ground-based interferometers," Phys. Rev. D85 (2012) 023534, arXiv:1109.0022 [astro-ph.C0]. [Erratum: Phys. Rev.D86,069901(2012)].

[184] L. Senatore, E. Silverstein, and M. Zaldarriaga, "New Sources of Gravitational Waves during Inflation," JCAP 1408 (2014) 016, arXiv:1109.0542 [hep-th].

[185] D. Carney, W. Fischler, E. D. Kovetz, D. Lorshbough, and S. Paban, "Rapid field excursions and the inflationary tensor spectrum," JHEP 11 (2012) 042, arXiv:1209.3848 [hep-th].

[186] M. Biagetti, M. Fasiello, and A. Riotto, "Enhancing Inflationary Tensor Modes through Spectator Fields," Phys. Rev. D88 (2013) 103518, arXiv:1305.7241 [astro-ph.CO].

[187] M. Biagetti, E. Dimastrogiovanni, M. Fasiello, and M. Peloso, "Gravitational Waves and Scalar Perturbations from Spectator Fields," JCAP 1504 (2015) 011, arXiv:1411.3029 [astro-ph.CO].

[188] C. Goolsby-Cole and L. Sorbo, "Nonperturbative production of massless scalars during inflation and generation of gravitational waves," JCAP 1708 no. 08, (2017) 005, arXiv:1705.03755 [astro-ph.CO].

[189] M. M. Anber and L. Sorbo, "Non-Gaussianities and chiral gravitational waves in natural steep inflation," Phys. Rev. D85 (2012) 123537, arXiv:1203.5849 [astro-ph.CO].

[190] N. Barnaby and M. Peloso, "Large Nongaussianity in Axion Inflation," Phys. Rev. Lett. 106 (2011) 181301, arXiv:1011.1500 [hep-ph].

[191] N. Barnaby, J. Moxon, R. Namba, M. Peloso, G. Shiu, and P. Zhou, "Gravity waves and non-Gaussian features from particle production in a sector gravitationally coupled to the inflaton," Phys. Rev. D86 (2012) 103508, arXiv:1206.6117 [astro-ph.CO].

[192] O. Ozsoy, "On Synthetic Gravitational Waves from Multi-field Inflation," JCAP 1804 no. 04, (2018) 062, arXiv:1712.01991 [astro-ph.C0]. 
[193] A. Maleknejad and M. M. Sheikh-Jabbari, "Gauge-flation: Inflation From Non-Abelian Gauge Fields," Phys. Lett. B723 (2013) 224-228, arXiv:1102.1513 [hep-ph].

[194] E. Dimastrogiovanni and M. Peloso, "Stability analysis of chromo-natural inflation and possible evasion of Lyth's bound," Phys. Rev. D87 no. 10, (2013) 103501, arXiv:1212.5184 [astro-ph.CO].

[195] P. Adshead, E. Martinec, and M. Wyman, "Gauge fields and inflation: Chiral gravitational waves, fluctuations, and the Lyth bound," Phys. Rev. D88 no. 2, (2013) 021302, arXiv:1301.2598 [hep-th].

[196] P. Adshead, E. Martinec, and M. Wyman, "Perturbations in Chromo-Natural Inflation," JHEP 09 (2013) 087, arXiv: 1305. 2930 [hep-th].

[197] I. Obata, T. Miura, and J. Soda, "Chromo-Natural Inflation in the Axiverse," Phys. Rev. D92 no. 6, (2015) 063516, arXiv:1412.7620 [hep-ph]. [Addendum: Phys. Rev.D95,no.10,109902(2017)].

[198] A. Maleknejad, "Axion Inflation with an SU(2) Gauge Field: Detectable Chiral Gravity Waves," JHEP 07 (2016) 104, arXiv:1604.03327 [hep-ph].

[199] E. Dimastrogiovanni, M. Fasiello, and T. Fujita, "Primordial Gravitational Waves from Axion-Gauge Fields Dynamics," JCAP 1701 no. 01, (2017) 019, arXiv:1608.04216 [astro-ph.CO].

[200] A. Agrawal, T. Fujita, and E. Komatsu, "Large Tensor Non-Gaussianity from Axion-Gauge Fields Dynamics," arXiv:1707.03023 [astro-ph.C0].

[201] P. Adshead and E. I. Sfakianakis, "Higgsed Gauge-flation," JHEP 08 (2017) 130, arXiv:1705.03024 [hep-th].

[202] R. R. Caldwell and C. Devulder, "Axion Gauge Field Inflation and Gravitational Leptogenesis: A Lower Bound on B Modes from the Matter-Antimatter Asymmetry of the Universe," Phys. Rev. D97 no. 2, (2018) 023532, arXiv:1706.03765 [astro-ph.CO].

[203] A. Agrawal, T. Fujita, and E. Komatsu, "Tensor Non-Gaussianity from Axion-Gauge-Fields Dynamics : Parameter Search," arXiv:1802.09284 [astro-ph.CO].

[204] J. R. Espinosa, D. Racco, and A. Riotto, "A Cosmological Signature of the SM Higgs Instability: Gravitational Waves," arXiv:1804.07732 [hep-ph].

[205] S. Endlich, A. Nicolis, and J. Wang, "Solid Inflation," JCAP 1310 (2013) 011, arXiv: 1210.0569 [hep-th]. 
[206] N. Bartolo, D. Cannone, A. Ricciardone, and G. Tasinato, "Distinctive signatures of space-time diffeomorphism breaking in EFT of inflation," JCAP 1603 no. 03, (2016) 044, arXiv:1511.07414 [astro-ph.C0].

[207] A. Ricciardone and G. Tasinato, "Primordial gravitational waves in supersolid inflation," Phys. Rev. D96 no. 2, (2017) 023508, arXiv:1611.04516 [astro-ph.CO].

[208] A. Ricciardone and G. Tasinato, "Anisotropic tensor power spectrum at interferometer scales induced by tensor squeezed non-Gaussianity," JCAP 1802 no. 02, (2018) 011, arXiv:1711.02635 [astro-ph.CO].

[209] G. Domènech, T. Hiramatsu, C. Lin, M. Sasaki, M. Shiraishi, and Y. Wang, "CMB Scale Dependent Non-Gaussianity from Massive Gravity during Inflation," JCAP 1705 no. 05, (2017) 034, arXiv:1701.05554 [astro-ph.CO].

[210] G. Ballesteros, D. Comelli, and L. Pilo, "Massive and modified gravity as self-gravitating media," Phys. Rev. D94 no. 12, (2016) 124023, arXiv: 1603.02956 [hep-th].

[211] D. Cannone, J.-O. Gong, and G. Tasinato, "Breaking discrete symmetries in the effective field theory of inflation," JCAP 1508 no. 08, (2015) 003, arXiv:1505.05773 [hep-th].

[212] C. Lin and L. Z. Labun, "Effective Field Theory of Broken Spatial Diffeomorphisms," JHEP 03 (2016) 128, arXiv:1501.07160 [hep-th].

[213] D. Cannone, G. Tasinato, and D. Wands, "Generalised tensor fluctuations and inflation," JCAP 1501 no. 01, (2015) 029, arXiv: 1409.6568 [astro-ph.CO].

[214] M. Akhshik, "Clustering Fossils in Solid Inflation," JCAP 1505 no. 05, (2015) 043, arXiv:1409.3004 [astro-ph.C0].

[215] M. Biagetti, E. Dimastrogiovanni, and M. Fasiello, "Possible signatures of the inflationary particle content: spin-2 fields," JCAP 1710 no. 10, (2017) 038, arXiv:1708.01587 [astro-ph.C0].

[216] E. Dimastrogiovanni, M. Fasiello, and G. Tasinato, "Probing the inflationary particle content: extra spin-2 field," JCAP 1808 no. 08, (2018) 016, arXiv:1806.00850 [astro-ph.CO].

[217] T. Fujita, S. Kuroyanagi, S. Mizuno, and S. Mukohyama, "Blue-tilted Primordial Gravitational Waves from Massive Gravity," arXiv:1808.02381 [gr-qc].

[218] N. Bartolo et al., "Science with the space-based interferometer LISA. IV: Probing inflation with gravitational waves," JCAP 1612 no. 12, (2016) 026, arXiv:1610.06481 [astro-ph.CO]. 
[219] O. Seto, J. Yokoyama, and H. Kodama, "What happens when the inflaton stops during inflation," Phys. Rev. D61 (2000) 103504, arXiv:astro-ph/9911119 [astro-ph].

[220] S. M. Leach and A. R. Liddle, "Inflationary perturbations near horizon crossing," Phys. Rev. D63 (2001) 043508, arXiv:astro-ph/0010082 [astro-ph].

[221] S. M. Leach, M. Sasaki, D. Wands, and A. R. Liddle, "Enhancement of superhorizon scale inflationary curvature perturbations," Phys. Rev. D64 (2001) 023512, arXiv:astro-ph/0101406 [astro-ph].

[222] F. Finelli and R. Brandenberger, "On the generation of a scale invariant spectrum of adiabatic fluctuations in cosmological models with a contracting phase," Phys. Rev. D65 (2002) 103522, arXiv:hep-th/0112249 [hep-th].

[223] M. Gasperini and G. Veneziano, "The Pre - big bang scenario in string cosmology," Phys. Rept. 373 (2003) 1-212, arXiv:hep-th/0207130 [hep-th].

[224] S. Gratton, J. Khoury, P. J. Steinhardt, and N. Turok, "Conditions for generating scale-invariant density perturbations," Phys. Rev. D69 (2004) 103505, arXiv:astro-ph/0301395 [astro-ph].

[225] L. A. Boyle, P. J. Steinhardt, and N. Turok, "A New duality relating density perturbations in expanding and contracting Friedmann cosmologies," Phys. Rev. D70 (2004) 023504, arXiv:hep-th/0403026 [hep-th].

[226] Y.-S. Piao, "On the dualities of primordial perturbation spectrums," Phys. Lett. B606 (2005) 245-250, arXiv:hep-th/0404002 [hep-th].

[227] L. E. Allen and D. Wands, "Cosmological perturbations through a simple bounce," Phys. Rev. D70 (2004) 063515, arXiv:astro-ph/0404441 [astro-ph].

[228] J. Khoury and F. Piazza, "Rapidly-Varying Speed of Sound, Scale Invariance and Non-Gaussian Signatures," JCAP 0907 (2009) 026, arXiv:0811.3633 [hep-th].

[229] J. Khoury and G. E. J. Miller, "Towards a Cosmological Dual to Inflation," Phys. Rev. D84 (2011) 023511, arXiv:1012.0846 [hep-th].

[230] D. Battefeld and P. Peter, "A Critical Review of Classical Bouncing Cosmologies," Phys. Rept. 571 (2015) 1-66, arXiv:1406.2790 [astro-ph.CO].

[231] R. Brandenberger and P. Peter, "Bouncing Cosmologies: Progress and Problems," Found. Phys. 47 no. 6, (2017) 797-850, arXiv:1603.05834 [hep-th]. 
[232] S. Hirano, T. Kobayashi, and S. Yokoyama, "Ultra slow-roll G-inflation," Phys. Rev. D94 no. 10, (2016) 103515, arXiv:1604.00141 [astro-ph.Co].

[233] X. Chen, H. Firouzjahi, M. H. Namjoo, and M. Sasaki, "A Single Field Inflation Model with Large Local Non-Gaussianity," EPL 102 no. 5, (2013) 59001, arXiv:1301.5699 [hep-th].

[234] X. Chen, H. Firouzjahi, E. Komatsu, M. H. Namjoo, and M. Sasaki, "In-in and $\delta N$ calculations of the bispectrum from non-attractor single-field inflation," JCAP 1312 (2013) 039, arXiv:1308.5341 [astro-ph.CO].

[235] Y.-F. Cai, X. Chen, M. H. Namjoo, M. Sasaki, D.-G. Wang, and Z. Wang, "Revisiting non-Gaussianity from non-attractor inflation models," arXiv:1712.09998 [astro-ph.CO].

[236] J. A. Adams, B. Cresswell, and R. Easther, "Inflationary perturbations from a potential with a step," Phys. Rev. D64 (2001) 123514, arXiv:astro-ph/0102236 [astro-ph].

[237] J.-O. Gong, "Breaking scale invariance from a singular inflaton potential," JCAP 0507 (2005) 015, arXiv:astro-ph/0504383 [astro-ph].

[238] R. K. Jain, P. Chingangbam, L. Sriramkumar, and T. Souradeep, "The tensor-to-scalar ratio in punctuated inflation," Phys. Rev. D82 (2010) 023509, arXiv:0904.2518 [astro-ph.C0].

[239] A. Achucarro, J.-O. Gong, S. Hardeman, G. A. Palma, and S. P. Patil, "Features of heavy physics in the CMB power spectrum," JCAP 1101 (2011) 030, arXiv:1010.3693 [hep-ph].

[240] N. Bartolo, V. Domcke, D. G. Figueroa, J. Garcia-Bellido, M. Peloso, M. Pieroni, A. Ricciardone, M. Sakellariadou, L. Sorbo, and G. Tasinato, "Probing non-Gaussian Stochastic Gravitational Wave Backgrounds with LISA," arXiv:1806.02819 [astro-ph.CO].

[241] B. Thorne, T. Fujita, M. Hazumi, N. Katayama, E. Komatsu, and M. Shiraishi, "Finding the chiral gravitational wave background of an axion-SU(2) inflationary model using CMB observations and laser interferometers," Phys. Rev. D97 no. 4, (2018) 043506, arXiv: 1707.03240 [astro-ph.cO].

[242] O. Ozsoy, "On Synthetic Gravitational Waves from Multi-field Inflation," JCAP 1804 no. 04, (2018) 062, arXiv:1712.01991 [astro-ph.C0].

[243] M. Shiraishi, D. Nitta, and S. Yokoyama, "Parity Violation of Gravitons in the CMB Bispectrum," Prog. Theor. Phys. 126 (2011) 937-959, arXiv:1108.0175 [astro-ph.CO]. 
[244] H. W. H. Tahara and J. Yokoyama, "CMB B-mode auto-bispectrum produced by primordial gravitational waves," PTEP 2018 no. 1, (2018) 013E03, arXiv:1704.08904 [astro-ph.CO].

[245] T. Zhu, W. Zhao, Y. Huang, A. Wang, and Q. Wu, "Effects of parity violation on non-gaussianity of primordial gravitational waves in Horava-Lifshitz gravity," Phys. Rev. D88 (2013) 063508, arXiv: 1305.0600 [hep-th].

[246] Y. Huang, A. Wang, R. Yousefi, and T. Zhu, "Primordial non-Gaussianity of gravitational waves in Ho?ava-Lifshitz gravity," Phys. Rev. D88 no. 2, (2013) 023523, arXiv:1304.1556 [hep-th].

[247] J. L. Cook and L. Sorbo, "An inflationary model with small scalar and large tensor nongaussianities," JCAP 1311 (2013) 047, arXiv:1307.7077 [astro-ph.CO].

[248] J. Garcia-Bellido, M. Peloso, and C. Unal, "Gravitational Wave signatures of inflationary models from Primordial Black Hole Dark Matter," JCAP 1709 no. 09, (2017) 013, arXiv:1707.02441 [astro-ph.C0].

[249] N. Bartolo, V. De Luca, G. Franciolini, M. Peloso, D. Racco, and A. Riotto, "Testing Primordial Black Holes as Dark Matter through LISA," arXiv:1810.12224 [astro-ph.CO].

[250] N. Bartolo, V. De Luca, G. Franciolini, M. Peloso, and A. Riotto, "The Primordial Black Hole Dark Matter - LISA Serendipity," arXiv:1810.12218 [astro-ph.CO].

[251] D. Anninos, V. De Luca, G. Franciolini, A. Kehagias, and A. Riotto, "Cosmological Shapes of Higher-Spin Gravity," arXiv:1902.01251 [hep-th].

[252] X. Gao, T. Kobayashi, M. Shiraishi, M. Yamaguchi, J. Yokoyama, and S. Yokoyama, "Full bispectra from primordial scalar and tensor perturbations in the most general single-field inflation model," PTEP 2013 (2013) 053E03, arXiv:1207.0588 [astro-ph.CO].

[253] B. Allen and S. Koranda, "Temperature fluctuations in the cosmic background radiation from inflationary cosmological models," Phys. Rev. D50 (1994) 3713, arXiv:astro-ph/9404068 [astro-ph].

[254] X. Gao, T. Kobayashi, M. Yamaguchi, and J. Yokoyama, "Primordial non-Gaussianities of gravitational waves in the most general single-field inflation model," Phys. Rev. Lett. 107 (2011) 211301, arXiv:1108.3513 [astro-ph.CO].

[255] X. Gao, T. Kobayashi, M. Shiraishi, M. Yamaguchi, J. Yokoyama, and S. Yokoyama, "Full bispectra from primordial scalar and tensor 
perturbations in the most general single-field inflation model," PTEP 2013 (2013) 053E03, arXiv:1207.0588 [astro-ph.CO].

[256] J. Soda, H. Kodama, and M. Nozawa, "Parity Violation in Graviton Non-gaussianity," JHEP 08 (2011) 067, arXiv:1106.3228 [hep-th].

[257] P. McFadden and K. Skenderis, "Cosmological 3-point correlators from holography," JCAP 1106 (2011) 030, arXiv:1104.3894 [hep-th].

[258] M. H. Namjoo, H. Firouzjahi, and M. Sasaki, "Violation of non-Gaussianity consistency relation in a single field inflationary model," EPL 101 no. 3, (2013) 39001, arXiv:1210.3692 [astro-ph.CO].

[259] X. Chen, H. Firouzjahi, M. H. Namjoo, and M. Sasaki, "A Single Field Inflation Model with Large Local Non-Gaussianity," EPL 102 no. 5, (2013) 59001, arXiv:1301.5699 [hep-th].

[260] Q.-G. Huang and Y. Wang, "Large Local Non-Gaussianity from General Single-field Inflation," JCAP 1306 (2013) 035, arXiv:1303.4526 [hep-th].

[261] X. Chen, H. Firouzjahi, E. Komatsu, M. H. Namjoo, and M. Sasaki, "In-in and $\delta N$ calculations of the bispectrum from non-attractor single-field inflation," JCAP 1312 (2013) 039, arXiv:1308.5341 [astro-ph.C0].

[262] E. Dimastrogiovanni, M. Fasiello, G. Tasinato, and D. Wands, "Tensor non-Gaussianities from Non-minimal Coupling to the Inflaton," arXiv:1810.08866 [astro-ph.CO].

[263] G. Goon, K. Hinterbichler, A. Joyce, and M. Trodden, "Shapes of gravity: Tensor non-Gaussianity and massive spin-2 fields," arXiv:1812.07571 [hep-th].

[264] D. Babich, P. Creminelli, and M. Zaldarriaga, "The Shape of non-Gaussianities," JCAP 0408 (2004) 009, arXiv : astro-ph/0405356 [astro-ph].

[265] H. Motohashi and J. White, "Disformal invariance of curvature perturbation," JCAP 1602 no. 02, (2016) 065, arXiv:1504.00846 [gr-qc].

[266] J. Gleyzes, D. Langlois, F. Piazza, and F. Vernizzi, "Healthy theories beyond Horndeski," Phys. Rev. Lett. 114 no. 21, (2015) 211101, arXiv: 1404.6495 [hep-th].

[267] D. Langlois and K. Noui, "Degenerate higher derivative theories beyond Horndeski: evading the Ostrogradski instability," JCAP 1602 no. 02, (2016) 034, arXiv:1510.06930 [gr-qc].

[268] J. Ben Achour, M. Crisostomi, K. Koyama, D. Langlois, K. Noui, and G. Tasinato, "Degenerate higher order scalar-tensor theories beyond Horndeski up to cubic order," JHEP 12 (2016) 100, arXiv:1608.08135 [hep-th]. 
[269] R. Adler, M. Bazin, M. Schiffer, and C. Weichert, Introduction to General Relativity. International series in pure and applied physics. McGraw-Hill, 1965.

[270] A. Jeffrey and D. Zwillinger, Table of Integrals, Series, and Products. Elsevier Science, 2007.

[271] M. Abramowitz and I. Stegun, Handbook of Mathematical Functions: With Formulas, Graphs, and Mathematical Tables. Applied mathematics series. Dover Publications, 1965.

[272] F. Olver, N. I. of Standards, T. (U.S.), D. Lozier, R. Boisvert, and C. Clark, NIST Handbook of Mathematical Functions Hardback and CD-ROM. Cambridge University Press, 2010.

[273] N. Lebedev and R. Silverman, Special Functions and Their Applications. Dover Books on Mathematics. Dover Publications, 1972. 